\title{
Um resultado geral de modelo completude de expansões do corpo ordenado dos reais
}

Rodrigo Figueiredo

DissertaÇÃo APRESENTADA

AO

Instituto de MatemáticA e EstatísticA

DA

Universidade de SÃo PAUlo

PARA

OBTENÇÃA DO TÍTULO

DE

Mestre em CiênCIAS

Área de concentração: Matemática

Orientador: Prof. Dr. Ricardo Bianconi

São Paulo, Agosto de 2012 



\section{Um resultado geral de modelo completude de expansões do corpo ordenado dos reais}

Este exemplar corresponde à redação final da dissertação devidamente corrigida

e defendida por Rodrigo Figueiredo

e aprovada pela Comissão Julgadora.

Banca Examinadora:

- Prof. Dr. Ricardo Bianconi (orientador) - IME-USP.

- Prof. Dr. Hugo Luiz Mariano - IME-USP.

- Prof. Dr. Marcelo Esteban Coniglio - UNICAMP. 

A meus pais, Elza e Isaias 



\section{Agradecimentos}

A Deus, por tudo.

Aos meus pais, Elza e Isaias, pelo apoio incondicional em todas as circunstâncias. Vocês foram simplesmente fundamentais para a conclusão deste trabalho.

Ao meu irmão, Robson, que tem me auxiliado, de todas as formas, em todos os momentos, e à minha cunhada, Sara, pelos suporte e incentivo constantes.

À minha irmã, Jackceley, e ao meu cunhado, Lelis Gerson, por estarem sempre ao meu lado.

A Bárbara, Giselle, Maria Fernanda, Carlos, Jeremy e Wagner, pela inestimável amizade.

A Luciana, pela voluntária e valiosa correção de ordens ortográfica e gramatical da dissertação.

Aos professores Hugo Luiz Mariano e Marcelo Esteban Coniglio, membros da comissão julgadora, pelas preciosas sugestões, críticas e correções a este trabalho.

A Ofélia Alas, pela amizade e pelo estímulo, desde a iniciação científica.

Ao meu orientador, Ricardo Bianconi, pela amizade, pela paciência incalculável, pela disposição e pelos conselhos.

Indubitavelmente, vocês tornaram tudo isto possível. 



\section{Resumo}

Este trabalho tem como foco principal estabelecer condições gerais suficientes para que uma expansão do corpo ordenado dos reais por funções com domínio em $\mathbb{R}^{n}$ seja modelo completa e o-minimal. Para tanto, faremos uma abordagem sob o ponto de vista de estruturas fracas o-minimais, conforme o trabalho de Charbonnel e Wilkie. Além disso, ao analisar condições adicionais, podemos obter a seguinte generalização de um trabalho de Gabrielov: uma expansão o-minimal do corpo ordenado dos reais por funções $\mathcal{C}^{\infty}$ restritas, que é polinomialmente limitada e fechada sob diferenciação parcial, é modelo completa.

Palavras-chave: teoria modelo completa, estrutura o-minimal, expansão do corpo ordenado dos reais, estrutura fraca o-minimal, fecho Charbonnel 


\section{Abstract}

The main focus of this dissertation lies in establishing some general sufficient conditions for an expansion of the real ordered field by functions with domains $\mathbb{R}^{n}$ to be model complete and o-minimal. We approach this subject from the point of view of the o-minimal weak structures, by following the work of Charbonnel and Wilkie. Furthermore, when considering additional conditions, we are able to obtain the following generalization of a Gabrielov's result: an expansion of the real ordered field by restricted $\mathcal{C}^{\infty}$-functions, which is polynomially bounded and closed under partial differentiation, is model complete.

Keywords: model complete theory, o-minimal structure, expansion of the real ordered field, Charbonnel closure 


\section{Sumário}

$\begin{array}{ll}\text { Introdução } & 1\end{array}$

1 Introdução à teoria de modelos $\quad 5$

1.1 Estruturas e teorias . . . . . . . . . . . . . . . . . . 5

1.2 Conjuntos definíveis . . . . . . . . . . . . . . . . . . . . . . . . . 11

1.3 Modelo completude . . . . . . . . . . . . . . . . . . . . . 13

$\begin{array}{llr}2 & \text { Teorema do complemento } & 17\end{array}$

2.1 Estruturas fracas o-minimais . . . . . . . . . . . . . . . . . . 18

2.2 O Teorema da Decomposição Celular (TDC) . . . . . . . . . . . . . . . . . 54

2.3 Seleção de uma função de classe $\mathcal{C}^{p} \ldots$. . . . . . . . . . . . . . . . . . . . . . 81

2.4 Definindo a fronteira . . . . . . . . . . . . . . . . . . . 110

2.5 Condições suficientes para uma estrutura ser modelo completa e o-minimal . . 124

3 Condições alternativas para modelo completude $\quad 139$

3.1 Condições adicionais para uma estrutura ser modelo completa . . . . . . 139

$\begin{array}{ll}\text { Referências Bibliográficas } & 173\end{array}$ 
Índice Remissivo 


\section{Introdução}

A expansão do corpo ordenado dos reais $\overline{\mathbb{R}}=\langle\mathbb{R},+, \cdot, 0,1,<\rangle$ por símbolos de função como alvo de investigação de lógicos e matemáticos remonta aos meados do século XX. Em [20], por exemplo, Tarski demonstra que a teoria $T h(\overline{\mathbb{R}})$ de $\overline{\mathbb{R}}$ é decidível ${ }^{1}$ e, ao final, questiona se o mesmo vale para a teoria de $\mathbb{R}_{\exp }=\langle\mathbb{R},+, \cdot, 0,1,<, \exp \rangle$, em que $\exp (x)=e^{x}$ é a função exponencial real. Com a introdução da noção de estrutura o-minimal por Lou van den Dries, em [5], e o subsequente tratamento sistematizado destas estruturas apresentado por Knight, Pillay e Steinhorn, em [11] e [12], o interesse em expansões de $\overline{\mathbb{R}}$ deste tipo se intensificou. Desde então, tornaram-se mais frequentes resultados concernentes a expansões particulares, como [22], em que Wilkie prova que a teoria $T h\left(\mathbb{R}_{\exp }\right)$ é modelo completa, e a expansões de cunho mais geral, como [9], em que Gabrielov mostra que dada uma coleção $\mathcal{F}$ de funções analíticas a valores reais cujos domínios são vizinhanças abertas de $[-1,1]^{n}$ em $\mathbb{R}^{n}$, para variados $n$ 's, tal que $\mathcal{F}$ seja fechado sob diferenciação parcial, então a teoria da estrutura $\left\langle\overline{\mathbb{R}},\left\{f^{*}: f \in \mathcal{F}\right\}\right\rangle$ é modelo completa, sendo que $f^{*}: \mathbb{R}^{n} \rightarrow \mathbb{R}$ é definida por $f^{*}(\bar{x})=f(\bar{x})$, quando $\bar{x} \in[-1,1]^{n}$, e $f^{*}(\bar{x})=0$, caso $\bar{x} \in \mathbb{R}^{n} \backslash[-1,1]^{n}$. É neste entrecho que está inserido o cerne do presente trabalho.

Basicamente, a finalidade desta dissertação consiste em apresentar condições sobre a coleção dos conjuntos definíveis numa expansão $\widetilde{\mathbb{R}}=\langle\overline{\mathbb{R}}, R\rangle$, em que $R$ é um conjunto arbitrário de funções $f: \mathbb{R}^{n} \rightarrow \mathbb{R}$ (para vários $n$ ), as quais garantam que a teoria $T h(\widetilde{\mathbb{R}})$ seja modelo completa e, consequentemente, $\widetilde{\mathbb{R}}$ seja o-minimal. Esta investigação acerca de tais condições sobre $\widetilde{\mathbb{R}}=\langle\overline{\mathbb{R}}, R\rangle$ é realizada de forma sistemática em [16], mas não de modo pormenorizado.

\footnotetext{
${ }^{1}$ Uma teoria $T$ numa linguagem $L$ é dita decidível se existe um algoritmo que decide, quando uma $L$-sentença $\phi$ é dada como entrada, se $T \models \phi$.
} 
Esta obra pode, então, ser vista como um estudo esmiuçado (baseado completamente no artigo [16] de Maxwell) do tema em questão. Ela é composta de três capítulos.

O primeiro deles tem como meta introduzir as noções fundamentais para a compreensão dos resultados desenvolvidos nos capítulos posteriores. Então, conceitos como o de linguagem, estrutura, teoria modelo completa, entre outros, são fixados nesta parte inicial.

O segundo capítulo encerra o escopo deste projeto e é dividido em cinco seções. Uma vez que a inspeção da modelo completude da expansão $\widetilde{\mathbb{R}}=\langle\overline{\mathbb{R}}, R\rangle$ é feita através de estruturas fracas o-minimais, então as quatro primeiras seções são destinadas ao desenvolvimento de propriedades de tais estruturas e constituem o arcabouço de nossa análise. Começamos, assim, na seção primeira, com a definição de estrutura fraca o-minimal. Uma estrutura fraca ominimal é uma sequência $\mathcal{S}=\left\langle\mathcal{S}_{n}: n \geq 1\right\rangle$ de coleções de subconjuntos de $\mathbb{R}^{n}$ com as seguintes propriedades: para cada $n, \mathcal{S}_{n}$ é fechada sob intersecção e bijeção linear; os conjuntos semialgébricos de $\mathbb{R}^{n}$ são elementos de $\mathcal{S}_{n}$; para cada $A \in \mathcal{S}_{n}, A=\pi_{m, n}[B]$, em que $\pi_{m, n}$ é a projeção nas primeiras $n$ coordenadas de $m$ e $B$ é um fechado de $\mathbb{R}^{m}$ que pertence a $\mathcal{S}_{m} ; A \times B \in \mathcal{S}_{n+m}$, sempre que $A \in \mathcal{S}_{n}$ e $B \in \mathcal{S}_{m}$; e, finalmente, para todo $A \in \mathcal{S}_{n}$ existe um natural $N$ tal que, dado um subespaço afim $X \subseteq \mathbb{R}^{n}$ arbitrário, $A \cap X$ tem no máximo $N$ componentes conexas. Seguindo o texto de Charbonnel ([3]) com modificações de Wilkie ([21]), define-se, a partir de $\mathcal{S}$, uma coleção especial $C h(\mathcal{S})$, denominada por Maxwell ([16]) fecho Charbonnel de $\mathcal{S}$. Sob a hipótese de que $\mathcal{S}$ é estrutura fraca o-minimal, segue que o fecho Charbonnel de $\mathcal{S}$ também o é. A partir de então, assume-se a condição de $C h(\mathcal{S})$ ser estrutura fraca o-minimal. Como consequência, segue que esta coleção é fechada sob intersecção e união finitas, projeção e fecho topológico. No restante da seção, são expostas peculiaridades do fecho Charbonnel de $\mathcal{S}$, que são usadas como lemas para resultados posteriores. Da mesma forma, fazemos para as Seções 2 e 3, que têm como destaques, respectivamente, o Teorema da Decomposição Celular e o Lema da Seleção de uma Curva $\mathcal{C}^{p}$ (i. e., os conjuntos $A$ de $C h(\mathcal{S}) \cap \mathbb{R}^{n+m}$ contêm gráficos de funções $\mathcal{C}^{p}$, desde que o interior de $\pi_{n+m}[A]$ seja não vazio). O primeiro teorema afirma que, se uma condição de fronteira for satisfeita - a saber, a que para todo $A \in C h(\mathcal{S})$, existe um conjunto $T$ em $C h(\mathcal{S})$ cujo interior é vazio e de sorte que $T$ contém a fronteira de $A$ - então, em particular, os elementos de $C h(\mathcal{S})$ podem ser particionados finitamente, tal que cada elemento da partição é um conjunto em $C h(\mathcal{S})$ que apresenta "bom comportamento", 
denominado célula. Daí, podemos inferir que $C h(\mathcal{S})$ é fechado sob a operação do complemento. Na quarta seção, buscamos, então, estabelecer em que circunstância a condição de fronteira é lograda. De fato, basta que $A \in C h(\mathcal{S})$ seja a projeção do conjunto de zeros de uma função $\mathcal{C}^{p}$, conforme assegura o Teorema do Complemento. Na quinta e última seção, aplicamos os resultados desenvolvidos nas seções precedentes. Assim, tomando a coleção $\mathcal{D}$ de todos os conjuntos definíveis sem quantificadores em $\widetilde{\mathbb{R}}$, mostramos que $\mathcal{D}$ é uma estrutura fraca. Se supusermos que $R$ é constituído de funções $\mathcal{C}^{1}$, então, juntamente com o fato de que toda fórmula existencial (nas variáveis livres $\bar{x}$, digamos) da linguagem de $\widetilde{\mathbb{R}}$ é equivalente a uma da forma $\exists \vec{y} F(\bar{x}, \vec{y})=0$, em que $F$ é um polinômio nas novas funções e nas variáveis indicadas $(\bar{x}$ e $\vec{y})$, tem-se que os elementos de $\mathcal{D}$ são projeções de conjuntos de zeros de funções $\mathcal{C}^{1}$ em $\mathcal{D}$ (portanto, projeções de fechados em $\mathcal{D}$ ). Além disso, assumindo que a intersecção de qualquer elemento de $\mathcal{D}$ com um subconjunto afim arbitrário $X$ possui um número finito de componentes conexas, concluímos que $\mathcal{D}$ é uma estrutura fraca o-minimal indexestrutura! fraca o-minimal. Por conseguinte, seu fecho Charbonnel $C h(\mathcal{D})$ é também uma estrutura fraca o-minimal. Por último, ao nos valer também de que o fecho topológico dos conjuntos existencialmente definíveis em $\widetilde{\mathbb{R}}$ é existencialmente definível, chegamos que $C h(\mathcal{D})$ é composto apenas por projeções de conjuntos de zeros. Em suma, $C h(\mathcal{D})$ é uma estrutura fraca o-minimal cujos elementos são conjuntos da forma $\{\bar{x}: \exists \vec{y} f(\bar{x}, \vec{y})=0\}$, sendo que $f$ é uma função $\mathcal{C}^{p}$ em $C h(\mathcal{D})$. Portanto, a condição de fronteira é satisfeita e, assim, o Teorema da Decomposição Celular é válido para $C h(\mathcal{D})$. Desta última, segue imediatamente que $C h(\mathcal{D})$ é fechado sob o complemento e, então, $T h(\widetilde{\mathbb{R}})$ é modelo completa. Para a o-minimalidade de $\widetilde{\mathbb{R}}$, note que, um conjunto $A$ definível em $\widetilde{\mathbb{R}}$ arbitrário é um conjunto existencialmente definível em $\widetilde{\mathbb{R}}$, já que, pela modelo completude de $T h(\widetilde{\mathbb{R}})$, a fórmula que define $A$ é equivalente, em $\widetilde{\mathbb{R}}$, a uma fórmula existencial. Ou seja, $A \in C h(\mathcal{D})$. Portanto, $A$ tem um número finito de componentes conexas. Em outros termos, $A$ é união finita de intervalos e pontos.

No último capítulo, como consequência da tentativa de obter a modelo completude de redutos de estruturas o-minimais, investigamos condições adicionais que nos permitam enfraquecer a condição "o fecho topológico dos conjuntos existencialmente definíveis em $\widetilde{\mathbb{R}}$ é existencialmente definível", de sorte que façam referência apenas ao fecho de conjuntos definíveis sem quantificadores. 


\section{Capítulo 1}

\section{Introdução à teoria de modelos}

O alvo deste capítulo inicial consiste apenas em fixar os conceitos fundamentais para este trabalho, que são o de modelo completude de uma teoria e o de o-minimalidade de uma estrutura. As consequências destas propriedades não serão desenvolvidas aqui. Todavia, se houver algum interesse nos efeitos de tais particularidades sobre estruturas ou teorias por parte do leitor, este poderá consultar os textos [15] e [6], os quais tratam de forma mais abrangente este assunto.

As definições, teoremas e fatos apresentados neste capítulo foram extraídos dos livros [15], [2], [18] e das notas de aula [1].

\subsection{Estruturas e teorias}

A seguir, faremos uma exposição bastante sucinta de noções básicas como linguagem, estrutura, fórmula e teoria, que são subjacentes às concepções de o-minimalidade e modelo completude definidas nas próximas seções desta primeira parte do trabalho.

Definição 1.1.1. Uma assinatura é um conjunto $L=\mathcal{F} \cup \mathcal{R} \cup \mathcal{C}$ tal que $\mathcal{C}, \mathcal{F}$ e $\mathcal{R}$ são conjuntos dois a dois disjuntos.

Os elementos de $\mathcal{C}, \mathcal{F}$ e de $\mathcal{R}$ são chamados, respectivamente, de símbolos de constante, símbolos de função (ou símbolos funcionais) e símbolos predicativos (ou símbolos de relação). 
Cada símbolo predicativo $R$ e funcional $f$ é equipado com um número natural, $n_{R}$ e $n_{f}$, respectivamente, chamado aridade. Assim, dizemos que $f$ é um símbolo funcional $n_{f}$-ário e $R$ é um símbolo predicativo $n_{R}$-ário.

Definição 1.1.2. Uma linguagem de primeira ordem consiste num alfabeto que contém os símbolos $\wedge, \vee, \neg, \exists, \forall,($, ) e o símbolo de igualdade $=$; um conjunto enumerável de símbolos de variáveis $\operatorname{Var}=\left\{v_{n}: n \in \omega\right\}$; símbolos (não lógicos) de uma assinatura $L$ e , por fim, regras (gramaticais) de formação de expressões bem fundadas.

Além de $v_{1}, v_{2}, \ldots$, usaremos $w, x, y, z, \ldots$, como símbolos de variáveis. Como o que muda de uma linguagem para outra é apenas a assinatura $L$, usaremos, portanto, o símbolo $L$ para denotar também a linguagem de primeira ordem assim obtida.

Definição 1.1.3. Uma $L$-estrutura $\mathcal{M}$ é dada por:

1. um conjunto não vazio $M$ chamado de domínio (ou universo) de $\mathcal{M}$.

2. uma função $f^{\mathcal{M}}: M^{n_{f}} \rightarrow M$, para cada $f \in \mathcal{F}$.

3. um conjunto $R^{\mathcal{M}} \subseteq M^{n_{R}}$, para cada $R \in \mathcal{R}$.

4. um elemento $c^{\mathcal{M}} \in M$, para cada $c \in \mathcal{C}$.

E é denotada por $\mathcal{M}=\left\langle M,\left(f^{\mathcal{M}}\right)_{f \in \mathcal{F}},\left(R^{\mathcal{M}}\right)_{R \in \mathcal{R}},\left(c^{\mathcal{M}}\right)_{c \in \mathcal{C}}\right\rangle$.

As funções $f^{\mathcal{M}}$, relações $R^{\mathcal{M}}$ e constantes $c^{\mathcal{M}}$ são chamadas de interpretações (em $\mathcal{M}$ ) dos símbolos $f, R$ e $c$, respectivamente.

Por simplicidade de notação, uma estrutura $\mathcal{M}$ é, geralmente, denotada apenas pelo seu domínio $M$.

Definição 1.1.4. Sejam $M$ e $N$ duas $L$-estruturas. Um $L$-morfismo é uma função $\eta: M \rightarrow N$ tal que:

1. $\eta\left(f^{M}\left(a_{1}, \ldots, a_{n_{f}}\right)\right)=f^{M}\left(\eta\left(a_{1}\right), \ldots, \eta\left(a_{n_{f}}\right)\right)$, para todo $f \in \mathcal{F}$ e $\bar{a} \in M$. 
2. $\left(a_{1}, \ldots, a_{n_{R}}\right) \in R^{M} \Rightarrow\left(\eta\left(a_{1}\right), \ldots, \eta\left(a_{n_{R}}\right)\right) \in R^{N}$, para todo $R \in \mathcal{R}$ e $\bar{a} \in M$.

3. $\eta\left(c^{M}\right)=c^{N}$, para todo $c \in \mathcal{C}$.

Quando $\eta$ é injetora, dizemos que tal função é uma $L$-imersão.

Quando $\eta$ é bijetora, dizemos que $\eta$ é um $L$-isomorfismo.

Se $M \subseteq N$ e a função inclusão é um $L$-morfismo, dizemos que $M$ é uma subestrutura de $N$ ou que $N$ é uma extensão de $M\left(M \subseteq_{e} N\right)$.

Definição 1.1.5. Uma expressão $t$ em $L$ é um termo se existe uma sequência $t_{1}, \ldots, t_{n}$ tal que $t_{n}=t$ e para cada $t_{i}$ :

- $t_{i} \in \operatorname{Var}, \mathrm{ou}$

- $t_{i} \in \mathcal{C}$, ou

- $t_{i}$ é igual a $f\left(t_{i_{1}}, \ldots, t_{i_{j}}\right)$, com $f$ símbolo funcional $j$-ário e $i_{1}, \ldots, i_{j}<i$ dois a dois distintos.

Chamamos de complexidade do termo $t$ o menor natural $l(t)$ tal que existe uma sequência como a da definição acima.

Definição 1.1.6. Sejam $t$ um termo, com variáveis contidas em $\left\{v_{i_{1}}, \ldots, v_{i_{n}}\right\}$, e $\bar{a}=\left(a_{i_{1}}, \ldots, a_{i_{n}}\right) \in$ $M^{n}$, então definimos $t^{M}(\bar{a})$ do seguinte modo:

1. Se $t$ é um símbolo de constante $c$, então $t^{M}(\bar{a})=c^{M}$.

2. Se $t$ é uma variável $v_{i_{j}}$, com $j \in\{1, \ldots, n\}$, então $t^{M}(\bar{a})=a_{i_{j}}$.

3. Se $t$ é da forma $f\left(t_{1}, \ldots, t_{n_{f}}\right)$, sendo que $f$ é um símbolo funcional e $t_{1}, \ldots, t_{n_{f}}$ são termos, então $t^{M}(\bar{a})=f^{M}\left(t_{1}^{M}(\bar{a}), \ldots, t_{n_{f}}^{M}(\bar{a})\right)$.

Definição 1.1.7. Uma expressão $\phi$ em $L$ é uma fórmula se existe uma sequência $\phi_{1}, \ldots, \phi_{n}$ tal que $\phi_{n}=\phi$ e para cada $\phi_{i}$ : 
- $\phi_{i}$ é igual a $t_{1}=t_{2}$, com $t_{1}$ e $t_{2}$ termos, ou

- $\phi_{i}$ é igual $R\left(t_{1}, \ldots, t_{n}\right)$, com $R$ símbolo relacional $n$-ário e $t_{1}, \ldots, t_{n}$ termos, ou

- $\phi_{i}$ é $\phi_{j} \wedge \phi_{k}$ ou $\phi_{j} \vee \phi_{k}$ ou $\neg \phi_{j}, \operatorname{com} j, k<i$, ou

- $\exists v \phi_{k}$ ou $\forall v \phi_{j}$, com $v \in \operatorname{Var}$ e $j<i$.

Denominamos complexidade da fórmula $\phi$ o menor natural $l(\phi)$ tal que existe uma sequência como a definida logo acima.

As fórmulas do tipo $t_{1}=t_{2}$ e $R\left(t_{1}, \ldots, t_{n}\right)$ são chamadas de fórmulas atômicas.

Dizemos que uma variável $v$ ocorre livre numa fórmula $\phi$ se não está no escopo dos quantificadores $\exists v$ e $\forall v$ ou não for ocorrência nas expressões " $\exists v$ " e " $\forall v$ ". Caso contrário, dizemos que ela é ligada.

Uma fórmula é dita ser uma sentença se não possui variáveis livres.

Definição 1.1.8. Seja $\phi$ uma fórmula com variáveis livres de $t$ contidas em $\bar{v}=\left(v_{i_{1}}, \ldots, v_{i_{n}}\right)$ e seja $\bar{a}=\left(a_{i_{1}}, \ldots, a_{i_{n}}\right) \in M^{n}$. Definimos $M \models \phi(\bar{a})$ indutivamente por:

1. se $\phi$ é $t_{1}=t_{2}$, então: $M \models \phi(\bar{a})$ se, e somente se $t_{1}^{M}(\bar{a})=t_{2}^{M}(\bar{a})$;

2. se $\phi$ é $R\left(t_{1}, \ldots, t_{n_{R}}\right)$, então: $M \models \phi(\bar{a})$ se, e somente se $\left(t_{1}^{M}(\bar{a}), \ldots, t_{n_{R}}^{M}(\bar{a})\right) \in R^{M}$;

3. se $\phi$ é $\neg \psi$, então: $M \models \phi(\bar{a})$ se, e somente se $M \not \models \psi(\bar{a})$;

4. se $\phi$ é $(\theta \wedge \psi)$, então: $M \models \phi(\bar{a})$ se, e somente se $M \models \theta(\bar{a})$ e $M \models \psi(\bar{a})$;

5. se $\phi$ é $(\theta \vee \psi)$, então: $M \models \phi(\bar{a})$ se, e somente se $M \models \theta(\bar{a})$ ou $M \models \psi(\bar{a})$;

6. se $\phi$ é $\exists v_{j} \psi\left(\bar{v}, v_{j}\right)$, então: $M \models \phi(\bar{a})$ se, e somente se existe $b \in M$ tal que $M \models \psi(\bar{a}, b)$;

7. se $\phi$ é $\forall v_{j} \psi\left(\bar{v}, v_{j}\right)$, então: $M \models \phi(\bar{a})$ se, e somente se para todo $b \in M$ temos que $M \models \psi(\bar{a}, b)$.

Se $M \models \phi(\bar{a})$ dizemos que $M$ satisfaz $\phi(\bar{x})$, para $\bar{a} \in M$. 
Sejam $L$ e $L_{1}$ linguagens tal que $L \subset L_{1}$. Se $M_{1}$ é uma $L_{1}$-estrutura, então desprezando as interpretações dos símbolos em $L_{1} \backslash L$, obtemos uma $L$-estrutura $M$. $M$ é, então, chamada de reduto de $M_{1}$ e $M_{1}$ é denominada uma expansão de $M$.

\section{Notação.}

(a) $\bigwedge_{i=1}^{n} \phi_{i}$ abrevia $\phi_{1} \wedge \ldots \wedge \phi_{n}$

(b) $\bigvee_{i=1}^{n} \phi_{i}$ abrevia $\phi_{1} \vee \ldots \vee \phi_{n}$

Dizemos que uma $L$-fórmula $\phi$, com sequência de construção (de comprimento mínimo) $\phi_{1}, \ldots, \phi_{n}$, é aberta (ou livre de quantificadores ou ainda sem quantificadores) se para todo $i=1, \ldots, n, \phi_{i}$ é diferente de $\exists x \phi_{j}$ e de $\forall x \phi_{j}(j<i)$.

Uma fórmula é chamada de prenexa se é da forma $Q_{1} v_{1} \cdots Q_{n} v_{n} \phi$, de sorte que $n \geq 0, \phi$ é aberta e cada $Q_{i}$ é $\forall$ ou $\exists$. No caso de $Q_{i}=\exists$ (para todo $i$ ), tal fórmula é dita existencial. E, no caso de $Q_{i}=\forall$ (para todo $i$ ), é chamada de universal.

Teorema 1.1.9. Toda L-fórmula $\phi$ é equivalente, em qualquer L-estrutura, à alguma fórmula na forma prenexa $\phi^{\prime}$ (ou seja, $\left.\vdash \phi \leftrightarrow \phi^{\prime}\right)$.

Demonstração. A demonstração deste teorema pode ser encontrada em [18], págs. 37-38.

Um conjunto $T$ de sentenças $\phi$ de uma linguagem $L$ é chamado de $L$-teoria. Dizemos que $M$ é um modelo de $T(M \models T)$ se $M \models \phi$, para todo $\phi \in T$.

Uma $L$-teoria $T$ é dita consistente se não existe uma sentença $\phi$ tal que $T \vdash(\phi \wedge \neg \phi)^{1}$. Decorre do Teorema da Completude de Gödel ${ }^{2}$, que uma teoria $T$ é consistente se, e somente se, $T$ tem modelo.

\footnotetext{
${ }^{1} \mathrm{Ou}$ seja, $T$ não prova a sentença $\phi$ e sua negação $\neg \phi$.

2"Sejam $T$ uma $L$-teoria e $\phi$ uma $L$-sentença, então $T=\phi \Leftrightarrow T \vdash \phi$ ".
} 
Definição 1.1.10. Sejam $T$ uma $L$-teoria e $\phi\left(v_{1}, \ldots, v_{n}\right)$ uma fórmula de $L$. Então, $\phi$ é dita consequência lógica de $T(T \models \phi)$ se, para todo modelo $M$ de $T$ e para todo $\bar{a} \in M^{n}$, $M \models \phi(\bar{a})$.

Note, desta definição, que $T \models \phi$ é o mesmo que $T \models \forall \bar{v} \phi(\bar{v})$.

Uma $L$-teoria $T$ é fechada sob consequência lógica se, dada uma $L$-sentença $\phi$ tal que $T \models \phi$, obtemos $\phi \in T$.

Definição 1.1.11. Uma $L$-teoria consistente é dita completa se, para uma $L$-sentença $\phi$ arbitrária,

$$
\text { ou } T \models \phi \text { ou } T \models \neg \phi \text {. }
$$

Dizemos que uma $L$-teoria $T$ é maximal se, para toda sentença $\phi$, ou $\phi \in T$ ou $\neg \phi \in T$.

Fato. Uma L-teoria $T$ é maximal consistente se, e somente se é completa e fechada sob consequência lógica.

Seja $M$ uma $L$-estrutura. A teoria de $M$, designada por $T h(M)$, é o conjunto de todas as sentenças de $L$ que são válidas em $M$. Então, $T h(M)$ é completa e fechada sob consequência lógica.

É corriqueiro referir-se à "teoria de uma estrutura" em afirmações, porém utilizando apenas a expressão "estrutura". Assim, por exemplo, ao dizermos que "a estrutura $M$ é modelo completa" queremos dizer que "a teoria de $M$ é modelo completa".

Definição 1.1.12. Dizemos que uma classe $K$ de $L$-estruturas é uma classe elementar se existe uma $L$-teoria $T$ tal que $K=\{M: M \models T\}$.

Exemplo 1.1.13. Ordem linear densa. Seja $L=\{<\}$, em que $<$ é um símbolo de relação binário. A classe das ordens lineares densas é axiomatizada pelas seguintes sentenças:

- $\forall x \neg(x<x)$. 
- $\forall x \forall y \forall z((x<y \wedge y<z) \rightarrow x<z)$.

- $\forall x \forall y(x<y \vee x=y \vee y<x)$.

- $\forall x \forall y(x<y \rightarrow \exists z(x<z \wedge z<y))$.

Definição 1.1.14. Sejam $M$ e $N L$-estruturas. Uma função $j: M \rightarrow N$ é chamada de um $L$-morfismo elementar se

$$
M \models \phi\left(a_{1}, \ldots, a_{n}\right) \Leftrightarrow N \models \phi\left(j\left(a_{1}\right), \ldots, j\left(a_{n}\right)\right),
$$

para todas as $L$-fórmulas e todos $a_{1}, \ldots, a_{n} \in M$.

Se a função inclusão $\iota: M \rightarrow N$ é um morfismo elementar, então dizemos que $M$ é uma subestrutura elementar de $N$, ou equivalentemente, $N$ é uma extensão elementar de $M$. Neste caso, indicamos por $M \prec N$.

Fato. Todo L-morfismo elementar $j: M \rightarrow N$ se fatora, e de forma única, como $j=\iota \circ \mathrm{J}$, sendo que $J: M \rightarrow M^{\prime}$ é um L-isomorfismo $e \iota: M^{\prime} \hookrightarrow N$ é a inclusão elementar.

\subsection{Conjuntos definíveis}

Assim como em Geometria, onde os lugares geométricos desempenham um papel de destaque, distinguimos coleções de pontos de uma dada estrutura $M$ que têm em comum uma propriedade descrita por uma fórmula interpretada em $M$. A estas coleções damos o nome de conjuntos definíveis. Formalmente,

Definição 1.2.1. Seja $M$ uma $L$-estrutura. Dizemos que $X \subseteq M^{n}$ é definível se existem uma $L$-fórmula $\phi\left(v_{1}, \ldots, v_{n}, w_{1}, \ldots, w_{m}\right)$ e $\vec{b} \in M^{m}$ tal que $X=\left\{\bar{a} \in M^{n}: M \models \phi(\bar{a}, \vec{b})\right\}$. Neste caso, dizemos que $\phi(\vec{v}, \vec{b})$ define $X$.

Seja $A \subseteq M$. Dizemos que $X \subseteq M^{n}$ é definível com parâmetros em $A$ (ou $A$-definível) se existem uma fórmula $\phi\left(v_{1}, \ldots, v_{n}, w_{1}, \ldots, w_{l}\right)$ e $\vec{b} \in A^{l}$ tal que $\phi(\bar{v}, \vec{b})$ define $X$.

Se a fórmula $\phi$ da Definição 1.2 .1 é da forma $\exists \vec{y} \psi(\bar{x}, \vec{y})$, com $\psi(\bar{x}, \vec{y})$ aberta (ou seja, $\phi$ é 
uma fórmula existencial), então o conjunto $X$ definido, em $M$, por ela será dito $\exists_{1}$-definível ou existencialmente definível.

Exemplo 1.2.2. Seja $L_{r}=\{+,-, \cdot, 0,1\}$ a linguagem dos anéis e seja $M=\langle R,+,-, \cdot, 0,1\rangle$ um anel. Considere um polinômio $p(x) \in R[x]$. Portanto, a coleção $Y=\{x \in R: p(x)=0\}$ ( $i$. e., o conjunto das raízes de $p$ ) é definível. Além disso, se $a_{1}, \ldots, a_{n}$ são os coeficientes de $p$, então para qualquer subconjunto $A$ de $R$, tal que $\left\{a_{1}, \ldots, a_{n}\right\} \subseteq A$, segue que $Y$ é definível com parâmetros em $A$.

Podemos caracterizar os conjuntos definíveis de uma forma mais concreta ao tratar diretamente com as interpretações dos símbolos os quais ocorrem nas fórmulas que definem tais conjuntos.

Teorema 1.2.3. Seja $M$ uma L-estrutura. Suponha que $\mathcal{S}_{n}$ seja um conjunto de subconjuntos de $M^{n}$, para todo $n \geq 1$ e que $\mathcal{S}=\left\langle\mathcal{S}_{n}: n \geq 1\right\rangle$ seja o menor conjunto tal que:

1. $M^{n} \in \mathcal{S}_{n}$.

2. Para todos os simbolos funcionais $n$-ários $f \in \mathcal{F}$, o gráfico de $f^{M}$ está em $\mathcal{S}_{n+1}$.

3. Para todos os símbolos relacionais $n$-ários $R \in L, \mathcal{R}^{M} \in \mathcal{S}_{n}$.

4. Para quaisquer $i, j \leq n,\left\{\left(x_{1}, \ldots, x_{n}\right) \in M^{n}: x_{i}=x_{j}\right\} \in \mathcal{S}_{n}$.

5. Se $X \in \mathcal{S}_{n}$, então $M \times X \in \mathcal{S}_{n+1}$.

6. Cada $\mathcal{S}_{n}$ é fechado sob o complementar, a união e a intersecção.

7. Se $X \in \mathcal{S}_{n+1}$ e $\pi: M^{n+1} \rightarrow M^{n}$ é a projeção $\left(x_{1}, \ldots, x_{n+1}\right) \mapsto\left(x_{1}, \ldots, x_{n}\right)$, então $\pi[X] \in \mathcal{S}_{n}$.

8. Se $X \in \mathcal{S}_{n+m}$ e $\vec{b} \in M^{m}$, ent $\tilde{a} o\left\{\bar{a} \in M^{n}:(\bar{a}, \vec{b}) \in X\right\} \in \mathcal{S}_{n}$.

Então, $X \subseteq M^{n}$ é definível se, e somente se $X \in \mathcal{S}_{n}$.

Demonstração. Ver [15], págs. 22-23. 
Definição 1.2.4. Dizemos que uma estrutura $\langle M,<, \ldots\rangle$, em que $\langle M,<\rangle$ é uma ordem linear densa sem extremos ${ }^{3}$, é o-minimal se, para qualquer conjunto definível $X \subseteq M$, existem finitos intervalos abertos $I_{1}, \ldots, I_{n}$, com extremos em $M \cup\{ \pm \infty\}$, e um conjunto finito $X_{0}$ tal que $X=I_{1} \cup \cdots \cup I_{n} \cup X_{0}$.

Em outros termos, se $M$ é o-minimal, então os únicos subconjuntos definíveis de $M$ são os definidos apenas usando a ordem. Embora os subconjuntos definíveis de $M^{n}$ possam se mostrar, no caso de $M$ ser o-minimal, de um modo não tão simplista quanto os de $M$, ainda assim são "bem comportados".

\subsection{Modelo completude}

O estudo dos conjuntos definíveis torna-se menos complicado quando lidamos somente com fórmulas abertas. Por exemplo, na estrutura $\langle\mathbb{N},+, \cdot,<, 0,1\rangle$, os conjuntos definíveis sem quantificadores são expressos por equações e inequações polinomiais. Introduzimos, então, uma propriedade especial experimentada por certas teorias, a qual tem como consequência o fato de todo conjunto definível, em qualquer modelo desta teoria, poder ser definido por uma fórmula sem quantificadores.

Definição 1.3.1. Uma $L$-teoria $T$ admite eliminação de quantificadores se, para toda fórmula $\phi\left(x_{1}, \ldots, x_{n}\right)$ de $L$, existe uma fórmula aberta $\psi\left(x_{1}, \ldots, x_{n}\right)$ de $L$ tal que

$$
T \models \phi(\bar{x}) \leftrightarrow \psi(\bar{x})
$$

Dessa forma, fixados um modelo $M$ de uma teoria $T$, que admite eliminação de quantificadores, e um subconjunto $X \subseteq M$ definido por uma fórmula $\phi$, segue, pela definição 1.3.1, que existe uma fórmula aberta $\psi$, de sorte que $M \models \phi \leftrightarrow \psi$. Ou seja, $X$ pode ser definido por $\psi$.

Exemplo 1.3.2. A teoria dos corpos ordenados reais fechados (RCOF) admite eliminação de quantificadores na linguagem dos anéis ordenados.

\footnotetext{
${ }^{3} i$. e., é uma ordem linear densa em $M$ que não tem máximo e nem mínimo.
} 
Ocorre que algumas teorias $T$ não gozam desta peculiar propriedade de toda fórmula $\phi$ ser $T$-equivalente a uma aberta $\psi(i . \quad e ., T \models \phi \leftrightarrow \psi)$. Contudo, podem ter a característica de toda fórmula ser $T$-equivalente a uma fórmula que possui, em termos sintáticos, uma forma fixa, e. g. a fórmula existencial.

Definição 1.3.3. Uma $L$-teoria $T$ é modelo completa se, dados $M, N$ modelos quaisquer de $T$ temos que

$$
M \subseteq e N \Rightarrow M \prec N
$$

Há outras maneiras equivalentes de descrevermos a qualidade de uma teoria ser modelo completa. Exibimos algumas delas no teorema ulterior.

Definição 1.3.4. Seja $T$ uma $L$-teoria. Um modelo $M$ de $T$ é dito existencialmente fechado se, dados $N=T$, tal que $M \subseteq_{e} N$, e uma fórmula existencial (com parâmetros em $M$ ) $\exists \vec{y} \phi(\bar{x}, \vec{y})$, temos

$$
N \models \exists \vec{y} \phi(\bar{a}, \vec{y}) \Rightarrow M \models \exists \vec{y} \phi(\bar{a}, \vec{y})
$$

para $\bar{a} \in M$ e $\phi$ aberta.

Teorema 1.3.5. Seja T uma L-teoria consistente. São equivalentes:

(a) T é modelo completa.

(b) Para todo modelo $M$ de $T$, a teoria $T \cup \Delta_{M}$ é completa em $L_{M}$, sendo que $\Delta_{M}$ é o diagrama de $M$.

(c) Todo modelo de $T$ é existencialmente fechado.

(d) Para toda fórmula existencial $\phi\left(x_{1}, \ldots, x_{n}\right)$, existe uma fórmula universal $\psi\left(x_{1}, \ldots, x_{n}\right)$ de sorte que $T \models \phi\left(x_{1}, \ldots, x_{n}\right) \leftrightarrow \psi\left(x_{1}, \ldots, x_{n}\right)$.

(e) Para toda fórmula $\phi\left(x_{1}, \ldots, x_{n}\right)$ existe uma fórmula aberta $\psi\left(x_{1}, \ldots, x_{n}, y_{1}, \ldots, y_{m}\right)$ tal que $T \models \phi\left(x_{1}, \ldots, x_{n}\right) \leftrightarrow \forall y_{1} \cdots \forall y_{m} \psi\left(x_{1}, \ldots, x_{n}, y_{1}, \ldots, y_{m}\right)$.

Demonstração. Ver [2], pág. 187. 
Corolário 1.3.6. Seja T uma L-teoria consistente. São equivalentes:

(i) T é modelo completa.

(ii) Para toda fórmula $\phi\left(x_{1}, \ldots, x_{n}\right)$ existe uma fórmula aberta $\psi\left(x_{1}, \ldots, x_{n}, y_{1}, \ldots, y_{m}\right)$ tal que $T \models \phi\left(x_{1}, \ldots, x_{n}\right) \leftrightarrow \exists y_{1} \cdots \exists y_{m} \psi\left(x_{1}, \ldots, x_{n}, y_{1}, \ldots, y_{m}\right)$.

Demonstração. $(i) \Rightarrow(i i)$

De fato, dada uma fórmula $\phi(\bar{x})$, segue, de Teorema 1.3.5(e), que existe uma fórmula aberta $\psi(\bar{x}, \vec{y})$ tal que

$$
T \models \neg \phi(\bar{x}) \leftrightarrow \forall \psi(\bar{x}, \vec{y})
$$

Agora, note que

$$
T \models \forall \psi(\bar{x}, \vec{y}) \leftrightarrow \neg \exists \vec{y} \neg \psi(\bar{x}, \vec{y})
$$

Portanto,

$$
T \models \phi(\bar{x}) \leftrightarrow \exists \vec{y} \neg \psi(\bar{x}, \vec{y})
$$

$(i) \Leftarrow(i i)$

Análogo ao caso anterior.

Fica claro, a partir do item $(i i)$ do Corolário 1.3.6, que uma teoria que admite eliminação de quantificadores é modelo completa. Dessa forma, a modelo completude de uma teoria pode ser vista como uma versão fraca da eliminação de quantificadores.

Usaremos este critério, expresso neste corolário, para mostrar, na Seção 5 do Capítulo 2, a modelo completude de uma teoria especificada. 


\section{Capítulo 2}

\section{Teorema do complemento}

Faremos uma abordagem geométrica da o-minimalidade. Neste tratamento, a noção de estrutura o-minimal (sobre o corpo dos reais) é descrita da seguinte forma.

Definição 2.0.7 (Wilkie). Uma estrutura (em $\mathbb{R}$ ) é uma sequência $\mathcal{S}=\left\langle\mathcal{S}_{n}: n \geq 1\right\rangle$, em que cada $\mathcal{S}_{n}$ é uma coleção de subconjuntos de $\mathbb{R}^{n}$ tal que:

(E1) $\mathcal{S}_{n}$ é uma álgebra booleana de conjuntos, $i$. e., é fechado sob união, intersecção e complemento e contém os conjuntos $\mathbb{R}^{n}$ e $\emptyset$.

(E2) $\mathcal{S}_{n}$ contém todo subconjunto semi-algébrico ${ }^{1}$ de $\mathbb{R}^{n}$.

(E3) Se $A \in \mathcal{S}_{n}$ e $B \in \mathcal{S}_{m}$, então $A \times B \in \mathcal{S}_{n+m}$.

(E4) Se $m \geq n$ e $A \in \mathcal{S}_{m}$, então $\pi[A] \in \mathcal{S}_{n}$, sendo que $\pi: \mathbb{R}^{m} \rightarrow \mathbb{R}^{n}$ é a projeção nas $n$ primeiras coordenadas.

$S$ é chamada de estrutura o-minimal se

(E5) A fronteira de todo conjunto em $\mathcal{S}_{1}$ é finita.

\footnotetext{
${ }^{1}$ Veja a Definição 2.1.2.
} 
Todavia, conforme aponta Wilkie em [23], as operações de formação de conjunto em (E1) e (E4), precisamente, o complemento e a projeção, quando iteradas por um número de vezes grande o suficiente, podem gerar conjuntos inconvenientes. Trabalharemos, então, com uma versão enfraquecida de estrutura, chamada estrutura fraca, na qual os $\mathcal{S}_{n}$ 's não são necessariamente fechados sob tais operações. Por outro lado, fortalerecemos a condição de o-minimalidade ao impor que: para cada $A \in \mathcal{S}$, existe um natural $N$ de sorte que todos os conjuntos da forma $A \cap X$, em que $X$ é um subespaço afim de $\mathbb{R}^{n}$, têm no máximo $N$ componentes conexas.

Fixada uma estrutura fraca o-minimal $\mathcal{S}$, podemos obter, a partir desta, uma estrutura fraca $C h(\mathcal{S})$, que é também o-minimal e é fechada sob as operações de união finita, intersecção finita, projeção e fecho topológico. Ela é denominada, em alguns textos ${ }^{2}$, fecho Charbonnel de $\mathcal{S}$. Os elementos de $C h(\mathcal{S})$, no caso em que $\mathcal{S}$ é uma estrutura fraca o-minimal, possuem ainda outras propriedades como a equivalência entre interior vazio e medida nula e a seleção de uma função $\mathcal{C}^{p}$. Contudo, visto que o fecho Charbonnel não é necessariamente fechado sob o complemento, a propriedade de decomposição celular (Seção 2.2) só é válida para $C h(\mathcal{S})$ com a seguinte ressalva: a fronteira dos elementos do fecho Charbonnel deve estar contida num conjunto da coleção $C h(\mathcal{S})$ cujo interior é vazio. Para os nossos propósitos, a decomposição celular exerce um papel fundamental, pois garante que $C h(\mathcal{S})$ seja fechado sob o complemento. Portanto, é imprescindível determinar que pré-condições são suficientes para que esta condição de fronteira valha. De fato, segundo o Teorema do Complemento, basta que os elementos de $C h(\mathcal{S})$ sejam projeções de conjuntos dos zeros de funções $\mathcal{C}^{p}$. São, deste modo, exatamente esses quesitos que nortearão a busca por condições sobre os conjuntos definíveis numa expansão $\langle\overline{\mathbb{R}},\{f\}\rangle_{f \in R}$, as quais assegurarão que a teoria desta estrutura seja modelo completa.

\subsection{Estruturas fracas o-minimais}

A pauta desta seção inicial reside em inserir de maneira formal os conceitos de estrutura fraca o-minimal $(\mathcal{S})$ e, por conseguinte, seu fecho Charbonnel $(C h(\mathcal{S}))$, referido na introdução deste capítulo. Além disso, verificamos que se $\mathcal{S}$ é uma estrutura fraca o-minimal, então

\footnotetext{
${ }^{2}$ Por exemplo, em [16].
} 
$C h(\mathcal{S})$ também o é. Como consequência, os elementos de $C h(\mathcal{S})$ são Lebesgue mensuráveis. Também, para qualquer elemento $A$ da coleção $C h(\mathcal{S}), A$ tem interior vazio se, e somente se $A$ tem medida nula.

Começamos fixando alguma notação.

\section{Notação.}

1. $\mathbb{N}^{+}$denota o conjunto dos naturais positivos.

2. Denotamos por $\pi_{m, n}$, com $m \geq n$, a projeção de $\mathbb{R}^{m}$ em $\mathbb{R}^{n}$ nas $n$ primeiras coordenadas, i. e., $\pi_{m, n}: \mathbb{R}^{m} \rightarrow \mathbb{R}^{n}$ e $\pi_{m, n}\left(x_{1}, \ldots, x_{m}\right)=\left(x_{1}, \ldots, x_{n}\right)$.

3. Denotamos por $\overline{\mathbb{R}}$ a estrutura usual dos reais $\langle\mathbb{R},+,-, \cdot, 0,1,<\rangle$. A linguagem L para $\overline{\mathbb{R}}$ é a dos anéis ordenados, ou seja, $L=\{+,-, \cdot, 0,1,<\}$. Fica subentendido que estamos trabalhando com esta linguagem, salvo se mencionarmos o contrário.

4. A menos que seja mencionado o contrário, $\bar{x}$ denota uma n-upla e $\vec{y}$ uma m-upla.

5. Se $A \subseteq \mathbb{R}^{n}$, então $\bar{A}$ denota seu fecho topológico e $\AA$ seu interior.

6. Dado $A \subseteq \mathbb{R}^{n}$, indicamos por $\delta(A)=\bar{A} \backslash \AA$ a fronteira de $A$. Então, $\bar{x} \in \delta(A)$ se, e somente se, para todo $V$ aberto de $\mathbb{R}^{n}$, com $\bar{x} \in V,(V \cap A) \neq \emptyset \neq V \cap\left(\mathbb{R}^{n} \backslash A\right)$.

7. Dados $A \subseteq \mathbb{R}^{n+1}$ e $\bar{x} \in \mathbb{R}^{n}$, denotamos por $A_{\bar{x}}=\{y \in \mathbb{R}:(\bar{x}, y) \in A\}$, a fibra acima de $\bar{x}$ em $A$.

8. Usaremos o símbolo " $\Rightarrow \Leftarrow "$ para indicar uma redução ao absurdo.

Definição 2.1.1. Uma pré-estrutura é uma sequência $\mathcal{S}=\left\langle\mathcal{S}_{n}: n \geq 1\right\rangle$, em que cada $\mathcal{S}_{n}$ é uma coleção de subconjuntos de $\mathbb{R}^{n}$.

Se $\mathcal{S}$ e $\mathcal{S}^{\prime}$ são pré-estruturas, então escrevemos $\mathcal{S} \subseteq \mathcal{S}^{\prime}$, ao invés de $\mathcal{S}_{m} \subseteq \mathcal{S}_{m}^{\prime}$, para qualquer $m \in \mathbb{N}^{+}$.

Também, ao escrevermos $A \in \mathcal{S}$, queremos dizer $A \in \mathcal{S}_{m}$, para algum $m \in \mathbb{N}^{+}$. 
Definição 2.1.2. Chamamos um subconjunto $A \subseteq \mathbb{R}^{n}$ de semi-algébrico se $A$ é um conjunto definível (com parâmetros) em $\overline{\mathbb{R}}$ livre de quantificadores, i. e., $A=\left\{\bar{a} \in \mathbb{R}^{n}: \overline{\mathbb{R}}=\phi(\bar{a}, \vec{b})\right\}$, sendo que $\vec{b} \in \mathbb{R}^{m}$ e $\phi$ é fórmula aberta de $L$.

Equivalentemente, os conjuntos semi-algébricos de $\mathbb{R}^{n}$ são uniões finitas de subconjuntos de $\mathbb{R}^{n}$ da forma $\left\{\bar{x} \in \mathbb{R}^{n}: p_{1}(\bar{x})=\cdots=p_{k}(\bar{x})=0 \wedge q_{1}(\bar{x})>0 \wedge \cdots \wedge q_{l}(\bar{x})>0\right\}$, com $p_{1}, \ldots, p_{k}, q_{1}, \ldots, q_{l} \in \mathbb{R}[\bar{x}]$.

Definição 2.1.3. Uma pré-estrutura $\mathcal{S}=\left\langle\mathcal{S}_{n}: n \geq 1\right\rangle$ é chamada de estrutura fraca (em $\mathbb{R})$ se, para quaisquer $n, m \geq 1$, as seguintes condições são satisfeitas:

(EF1) Se $A, B \in \mathcal{S}_{n}$, então $A \cap B \in \mathcal{S}_{n}$.

(EF2) $\mathcal{S}_{n}$ contém todo subconjunto semi-algébrico de $\mathbb{R}^{n}$.

(EF3) Se $A \in \mathcal{S}_{n}$ e $B \in \mathcal{S}_{m}$, então $A \times B \in \mathcal{S}_{n+m}$.

(EF4) Se $A \in \mathcal{S}_{n}$ e $\sigma: \mathbb{R}^{n} \rightarrow \mathbb{R}^{n}$ é uma bijeção linear, então $\sigma[A] \in \mathcal{S}_{n}$.

Note que, dada uma estrutura fraca $\mathcal{S}=\left\langle\mathcal{S}_{n}: n \in \mathbb{N}^{+}\right\rangle$, então $\mathbb{R} \in \mathcal{S}_{1}$, uma vez que $\mathbb{R}=\{a \in \mathbb{R}: \overline{\mathbb{R}} \models(a=a)\}, i$. e., $\mathbb{R}$ é semi-algébrico. Consequentemente, $\mathbb{R}^{n} \in \mathcal{S}_{n}$.

Considere $B \subseteq \mathbb{R}^{n}$ um conjunto da forma

$$
\left\{\bar{x} \in \mathbb{R}^{n}: \bigwedge_{i=1}^{n}\left|x_{i}-a_{i}\right|<\epsilon\right\} \text { ou }\left\{\bar{x} \in \mathbb{R}^{n}: \bigwedge_{i=1}^{n}\left|x_{i}-a_{i}\right| \leq \epsilon\right\}
$$

$\operatorname{com} \bar{a} \in \mathbb{R}^{n}$ e $\epsilon>0$.

No primeiro caso, $B$ é chamado de cubo aberto e, no segundo, $B$ é chamado de cubo fechado. Em ambos os casos, é fácil ver que $B$ é um conjunto semi-algébrico e, portanto, $B \in \mathcal{S}$, para qualquer estrutura fraca $\mathcal{S}$. Se não especificarmos se o cubo é aberto ou fechado, é porque não faz diferença no contexto.

Definição 2.1.4. Um conjunto $X \subseteq \mathbb{R}^{n}$ é denominado subespaço (ou conjunto) afim se existem uma $M$ matriz real $n \times n$ e um $\bar{a} \in \mathbb{R}^{n}$, tal que $X=\left\{\bar{x} \in \mathbb{R}^{n}: M \bar{x}=\bar{a}\right\}$. 
A partir da equação matricial $M \bar{x}=\bar{c}$, em que $M=\left[m_{i j}\right], \bar{x}=\left[x_{i}\right], \bar{c}=\left[c_{i}\right]$ e $1 \leq i, j \leq n$, podemos obter um polinômio $p(\bar{x}, \vec{y}) \in \mathbb{Z}[\bar{x}, \vec{y}]$ (de grau menor ou igual a 1 ), sendo que $\vec{y}$ é uma $m$-upla (e $\left.m=n^{2}+n\right)$, com a seguinte peculiaridade: dado qualquer subespaço afim $Z \subseteq \mathbb{R}^{n}$ existem parâmetros $b_{1}, \ldots, b_{m} \in \mathbb{R}$ tal que $Z=\left\{\bar{x} \in \mathbb{R}^{n}: p(\bar{x}, \vec{b})=0\right\}$. De fato, $M \bar{x}=\bar{c}$ é equivalente ao seguinte sistema linear homogêneo

$$
\begin{gathered}
m_{11} x_{1}+\cdots+m_{1 n} x_{n}-c_{1}=0 \\
\vdots \\
m_{n 1} x_{1}+\cdots+m_{n n} x_{n}-c_{n}=0
\end{gathered},
$$

que, por sua vez, é equivalente à equação

$$
\left(m_{11} x_{1}+\cdots+m_{1 n} x_{n}-c_{1}\right)^{2}+\cdots+\left(m_{n 1} x_{1}+\cdots+m_{n n} x_{n}-c_{n}\right)^{2}=0 .
$$

Então, basta tomar o polinômio

$$
p(\bar{x}, \vec{y})=\left(y_{1} x_{1}+\cdots+y_{n} x_{n}-y_{n^{2}+1}\right)^{2}+\cdots+\left(y_{(n-1) n+1} x_{1}+\cdots+y_{n^{2}} x_{n}-y_{n^{2}+n}\right)^{2} .
$$

Definição 2.1.5. Suponha $n \geq 1$ e $A \subseteq \mathbb{R}^{n}$. Então, $\gamma(A)$ denota o menor natural $N$ com a seguinte propriedade:

(OM) para todo $X \subseteq \mathbb{R}^{n}$ subespaço afim, $A \cap X=A_{1} \cup A_{2} \cup \ldots \cup A_{N}$, tal que $A_{1}, \ldots, A_{N} \subseteq \mathbb{R}^{n}$ são subconjuntos conexos.

Se tal $N$ não existe, escrevemos $\gamma(A)=\infty$.

Definição 2.1.6. Uma estrutura fraca $\mathcal{S}=\left\langle\mathcal{S}_{n}: n \geq 1\right\rangle$ é dita o-minimal se, além das propriedades (EF1)-(EF4), satisfaz as duas condições a seguir:

(EF5) Para todo $n \geq 1$ e $A \in \mathcal{S}_{n}, \gamma(A)<\infty$.

(EF6) Para todo $n \geq 1$ e $A \in \mathcal{S}_{n}$, existem $m \geq n$ e um fechado $B \in \mathcal{S}_{m}$ tal que $A=\pi_{m, n}[B]$.

Apresentamos, agora, uma profíqua equivalência da condição (EF5). 
Lema 2.1.7. São equivalentes:

1. Para $n \geq 1$ e $A \in \mathcal{S}_{n}, \gamma(A)<\infty$.

2. Para $n \geq 1$ e $A \in \mathcal{S}_{n}$, existe $N \in \mathbb{N}$ tal que: para qualquer $X \subseteq \mathbb{R}^{n}$ subespaço afim, $A \cap X$ tem no máximo $N$ componentes conexas.

Demonstração. Considere o seguinte resultado,

AfIrmaÇÃo. Seja $Y \subseteq \mathbb{R}^{n}$ não vazio. Suponhamos que $Y$ tenha $m$ componentes conexas $C_{1}, \ldots, C_{m} \subseteq Y$. Então, $Y$ não pode ser escrito como a união de $t$ conexos $A_{1}, \ldots, A_{t} \subseteq Y$, com $m>t$.

Prova da Afirmação. Suponha que existam $A_{1}, \ldots, A_{t}$ conexos de $Y$, com $t<m$ e tal que

$$
Y=A_{1} \cup \ldots \cup A_{t}
$$

Assim, por hipótese,

$$
C_{1} \cup \ldots \cup C_{m}=A_{1} \cup \ldots \cup A_{t}
$$

Como para todo $i \in\{1, \ldots, t\}$ existe um único $j_{i} \in\{1, \ldots, m\}$ tal que $A_{i} \subseteq C_{j_{i}}$, então

$$
C_{1} \cup \ldots \cup C_{m}=C_{j_{1}} \cup \ldots \cup C_{j_{t}}
$$

Logo, para cada $C_{i} \in\left\{C_{1}, \ldots, C_{m}\right\} \backslash\left\{C_{j_{1}}, \ldots, C_{j_{t}}\right\}$ temos

$$
\text { ou } C_{i}=\emptyset \text { ou } C_{i} \subseteq C_{j_{k}} \text {, para algum } k \in\{1, \ldots, t\} .(\Rightarrow \Leftarrow)
$$

Sejam $n \geq 1$ e $A \subseteq \mathbb{R}^{n}$.

$(1) \Rightarrow(2)$ 
Por hipótese, existe $\gamma(A) \in \mathbb{N}$ tal que, para $X \subseteq \mathbb{R}^{n}$ subespaço afim arbitrário,

$$
A \cap X=A_{1} \cup \ldots \cup A_{\gamma(A)}, \operatorname{com} A_{1}, \ldots, A_{\gamma(A)} \text { conexos. }
$$

Pela Afirmação, existem, no máximo $\gamma(A)$ componentes conexas de $A \cap X$.

$(2) \Rightarrow(1)$

Considere o subsequente conjunto

$$
\zeta=\left\{m \in \mathbb{N}: \forall X \subseteq \mathbb{R}^{n} \text { afim } \exists A_{1} \cdots \exists A_{m} \subseteq \mathbb{R}^{n} \text { conexos, } A \cap X=A_{1} \cup \cdots \cup A_{m}\right\}
$$

Por hipótese, $\zeta \neq \emptyset$. Logo, pela Afirmação, $\gamma(A)=\min \zeta$.

Aviso. Ao longo deste capítulo, ao mencionarmos que uma pré-estrutura satisfaz (EF5), queremos dizer que ela satisfaz a propriedade (EF5) propriamente rotulada, como na Definição 2.1.6, ou sua equivalente, como no Lema 2.1.7. Ficará claro através do contexto.

Definição 2.1.8. Seja $\mathcal{S}=\left\langle\mathcal{S}_{n}: n \geq 1\right\rangle$ uma pré-estrutura. Então, definimos as sequências $\mathcal{S}^{u}, \mathcal{S}^{p r}$ e $\mathcal{S}^{c l}$ como segue:

(1) $\mathcal{S}^{u}=\left\langle\mathcal{S}_{n}^{u}: n \geq 1\right\rangle, \operatorname{com} \mathcal{S}_{n}^{u}=\left\{\bigcup_{i=1}^{p} A_{i}: p \geq 1\right.$ e $\left.A_{1}, \ldots, A_{p} \in \mathcal{S}_{n}\right\}$

(2) $\mathcal{S}^{p r}=\left\langle\mathcal{S}_{n}^{p r}: n \geq 1\right\rangle, \mathrm{com} \mathcal{S}_{n}^{p r}=\left\{\pi_{m, n}[A]: m \geq n\right.$ e $\left.A \in \mathcal{S}_{m}\right\}$.

(3) $\mathcal{S}^{c l}=\left\langle\mathcal{S}_{n}^{c l}: n \geq 1\right\rangle$, com $\mathcal{S}_{n}^{c l}=\left\{A_{0} \cap \bigcap_{i=1}^{p} \bar{A}_{i}: p \geq 0\right.$ e $\left.A_{0}, \ldots, A_{p} \in \mathcal{S}_{n}\right\}$.

Em (3), se $p=0$, então $\mathcal{S}_{n}^{c l}=\left\{A_{0}\right\}$.

Lema 2.1.9. Se $\mathcal{S}$ é uma estrutura fraca, então $\mathcal{S}^{u}, \mathcal{S}^{p r}$ e $\mathcal{S}^{c l}$ também o são. Além disso, se $\mathcal{S}$ é o-minimal, então cada $\mathcal{S}^{u}, \mathcal{S}^{p r}$ e $\mathcal{S}^{c l}$ também o é.

Demonstração. $\mathcal{S}^{u}$ é estrutura fraca. 
(EF1) Sejam $A, B \in \mathcal{S}_{n}^{u}$. Então,

$$
A=\bigcup_{i=1}^{p} A_{i} \text { e } B=\bigcup_{j=1}^{p^{\prime}} B_{j}
$$

$\operatorname{com} A_{i}, B_{j} \in \mathcal{S}_{n}$ e $p, p^{\prime} \geq 1$. Logo,

$$
A \cap B=\bigcup_{i=1}^{p} A_{i} \cap \bigcup_{j=1}^{p^{\prime}} B_{j}=\bigcup_{i=1}^{p} \bigcup_{j=1}^{p^{\prime}} A_{i} \cap B_{j} .
$$

Por hipótese, $A_{i} \cap B_{j} \in \mathcal{S}_{n}^{u}$, para $i=1, \ldots, p$ e $j=1, \ldots, p^{\prime}$. Portanto,

$$
\bigcup_{i=1}^{p} \bigcup_{j=1}^{p^{\prime}} A_{i} \cap B_{j} \in \mathcal{S}_{n}^{u}
$$

(EF2) Seja $A \subseteq \mathbb{R}^{n}$ subconjunto algébrico qualquer. Então, por hipótese, $A \in \mathcal{S}_{n}$ e, portanto, $A \in \mathcal{S}_{n}^{u}$.

(EF3) Sejam $A \in \mathcal{S}_{n}^{u}$ e $B \in \mathcal{S}_{m}^{u}$. Então,

$$
A=\bigcup_{i=1}^{p} A_{i} \text { e } B=\bigcup_{j=1}^{p^{\prime}} B_{j}
$$

$\operatorname{com} A_{i} \in \mathcal{S}_{n}$ e $B_{j} \in \mathcal{S}_{m}$ e $p, p^{\prime} \geq 1$. Assim,

$$
A \times B=\bigcup_{i=1}^{p} A_{i} \times \bigcup_{j=1}^{p^{\prime}} B_{j}=\bigcup_{i=1}^{p} \bigcup_{j=1}^{p^{\prime}} A_{i} \times B_{j} .
$$

Por hipótese, $A_{i} \times B_{j} \in \mathcal{S}_{n+m}$, para $i=1, \ldots, p$ e $j=1, \ldots, p^{\prime}$. Logo,

$$
\bigcup_{i=1}^{p} \bigcup_{j=1}^{p^{\prime}} A_{i} \times B_{j} \in \mathcal{S}_{n+m}^{u}
$$


(EF4) Seja $A \in \mathcal{S}_{n}^{u}$. Então,

$$
A=\bigcup_{i=1}^{p} A_{i}, \text { com } A_{i} \in \mathcal{S}_{n} \text { e } p \geq 1
$$

Dada $\sigma: \mathbb{R}^{n} \rightarrow \mathbb{R}^{n}$ uma bijeção linear, temos, por hipótese, que $\sigma\left[A_{i}\right] \in \mathcal{S}_{n}$. Logo,

$$
\sigma[A]=\bigcup_{i=1}^{p} \sigma\left[A_{i}\right] \in \mathcal{S}_{n}^{u}
$$

$\mathcal{S}^{u}$ é o-minimal.

(EF5) Sejam $n \geq 1$ e $A \in \mathcal{S}_{n}^{u}$. Então,

$$
A=\bigcup_{i=1}^{p} A_{i}, \text { com } A_{i} \in \mathcal{S}_{n} \text { e } p \geq 1
$$

Por hipótese, para todo $i \in\{1, \ldots, p\}$, existe $N_{i} \in \mathbb{N}$ de sorte que, dado um subespaço afim $X \subseteq \mathbb{R}^{n}$,

$$
A_{i} \cap X=A_{1}^{i} \cup \ldots \cup A_{N_{i}}^{i}, \operatorname{com} A_{1}^{i}, \ldots, A_{N_{i}}^{i} \subseteq \mathbb{R}^{n} \text { conexos. }
$$

Além disso, $N_{i}$ é o menor natural, para $A_{i}$, com a propriedade $(\mathrm{OM})$.

Considere o natural $N_{1}+\ldots+N_{p}$. Logo, dado $X \subseteq \mathbb{R}^{n}$ afim, temos

$$
A \cap X=\left(\bigcup_{i=1}^{p} A_{i}\right) \cap X=\bigcup_{i=1}^{p}\left(A_{i} \cap X\right)=\bigcup_{i=1}^{p}\left(A_{1}^{i} \cup \ldots \cup A_{N_{i}}^{i}\right) .
$$

Basta, portanto, tomarmos $\gamma(A)$ menor ou igual a $N_{1}+\ldots+N_{p}$. 
(EF6) Sejam $n \geq 1$ e $A \in \mathcal{S}_{n}^{u}$. Então,

$$
A=\bigcup_{i=1}^{p} A_{i}, \text { com } A_{i} \in \mathcal{S}_{n} \text { e } p \geq 1 .
$$

Por hipótese, para cada $i=1, \ldots, p$, existem $m_{i} \geq n$ e $B_{i} \in \mathcal{S}_{m_{i}}$ fechado tal que

$$
A_{i}=\pi_{m_{i}, n}\left[B_{i}\right]
$$

Seja $m=\max \left\{m_{i}: i=1, \ldots, p\right\}$. Chamemos, para cada $i \in\{1, \ldots, p\}$,

$$
B_{i}^{\prime}=B_{i} \times \mathbb{R}^{m-m_{i}}
$$

Então, $B_{i}^{\prime} \in \mathcal{S}_{m}$, pois $B_{i} \in \mathcal{S}_{m_{i}}$ e $\mathbb{R}^{m-m_{i}} \in \mathcal{S}_{m-m_{i}}$. Além disso, $B_{i}^{\prime}$ é fechado. Assim,

$$
\begin{gathered}
B=\bigcup_{i=1}^{p} B_{i}^{\prime} \text { é fechado, } \\
B \in \mathcal{S}_{m}^{u}
\end{gathered}
$$

e

$$
A=\bigcup_{i=1}^{p} A_{i}=\bigcup_{i=1}^{p} \pi_{m_{i}, n}\left[B_{i}\right]=\bigcup_{i=1}^{p} \pi_{m_{i}, n}\left[B_{i}^{\prime}\right]=\pi_{m, n}[B] .
$$

$\underline{\mathcal{S}}^{p r}$ é estrutura fraca.

(EF1) Sejam $A, B \in \mathcal{S}_{n}^{p r}$. Então,

$$
A=\pi_{m, n}[C] \text { e } B=\pi_{p, n}[D], \text { com } C \in \mathcal{S}_{m}, D \in \mathcal{S}_{p} \text { e } m, p \geq n
$$


Suponha, sem perda de generalidade, que $p \geq m$. Então,

$$
\begin{aligned}
A \cap B & =\pi_{m, n}[C] \cap \pi_{p, n}[D] \\
& =\pi_{m+p-n, n}\left[C \times \mathbb{R}^{p-n}\right] \cap \pi_{m+p-n, n}\left[D \times \mathbb{R}^{m-n}\right] \\
& =\pi_{m+p-n, n}\left[\sigma\left[C \times \mathbb{R}^{p-n}\right]\right] \cap \pi_{m+p-n, n}\left[D \times \mathbb{R}^{m-n}\right] \\
& =\pi_{m+p-n, n}\left[\sigma\left[C \times \mathbb{R}^{p-n}\right] \cap\left[D \times \mathbb{R}^{m-n}\right]\right],
\end{aligned}
$$

sendo que $\sigma: \mathbb{R}^{m+p-n} \rightarrow \mathbb{R}^{m+p-n}$ é a permutação que troca a $(n+i)$-ésima coordenada pela $(p+i)$-ésima coordenada, para $i=1, \ldots, m-n$.

Como $\sigma$ é bijeção linear, então, por hipótese,

$$
\sigma\left[C \times \mathbb{R}^{p-n}\right] \in \mathcal{S}_{m+p-n}
$$

Além disso,

$$
\sigma\left[C \times \mathbb{R}^{p-n}\right] \cap\left(D \times \mathbb{R}^{m-n}\right) \in \mathcal{S}_{m+p-n}
$$

Portanto,

$$
A \cap B=\pi_{m+p-n, n}\left[\sigma\left[C \times \mathbb{R}^{p-n}\right] \cap\left(D \times \mathbb{R}^{m-n}\right)\right] \in \mathcal{S}_{m+p-n}^{p r}
$$

(EF2) Seja $A \subseteq \mathbb{R}^{n}$ semi-algébrico qualquer. Então, $A \in \mathcal{S}_{n}$, por hipótese. Por conseguinte, $A=\imath[A] \in \mathcal{S}_{n}^{p r}$, sendo que $\imath: \mathbb{R}^{n} \rightarrow \mathbb{R}^{n}$ é a função identidade.

(EF3) Sejam $A \in \mathcal{S}_{n}^{p r}$ e $B \in \mathcal{S}_{m}^{p r}$. Então,

$$
A=\pi_{p, n}\left[A^{\prime}\right] \text { e } B=\pi_{q, m}\left[B^{\prime}\right], \operatorname{com} A^{\prime} \in \mathcal{S}_{p}, B^{\prime} \in \mathcal{S}_{q}, p \geq n \text { e } q \geq m
$$

Assim,

$$
A \times B=\pi_{p, n}\left[A^{\prime}\right] \times \pi_{q, m}\left[B^{\prime}\right]=\left\{\begin{array}{cl}
\pi_{p+q, m+n}\left[\sigma\left[A^{\prime} \times B^{\prime}\right]\right], & \text { se } p-n \geq m \\
\text { ou } & \\
\pi_{q+m+n, m+n}\left[\sigma_{1}\left[A^{\prime} \times \mathbb{R}^{m+n-p} \times B^{\prime}\right]\right], & \text { se } p-n<m
\end{array},\right.
$$

sendo que $\sigma: \mathbb{R}^{p+q} \rightarrow \mathbb{R}^{p+q}$ é a permutação que troca a $n+i$-ésima coordenada pela $p+i$-ésima 
coordenada, para $i=1, \ldots, m$ e $\sigma_{1}: \mathbb{R}^{q+m+n} \rightarrow \mathbb{R}^{q+m+n}$ é a permutação que troca a $n+i$-ésima coordenada pela $m+n+i$-ésima coordenada, para $i=1, \ldots, m$.

Uma vez que, $\sigma$ e $\sigma_{1}$ são bijeções lineares, então, por hipótese,

$$
\sigma\left[A^{\prime} \times B^{\prime}\right] \in \mathcal{S}_{p+q} \text { e } \sigma_{1}\left[A^{\prime} \times \mathbb{R}^{m+n-p} \times B^{\prime}\right] \in \mathcal{S}_{q+m+n}
$$

Portanto, $A \times B \in \mathcal{S}_{m+n}^{p r}$.

(EF4) Sejam $A \in \mathcal{S}_{n}^{p r}$ e $\sigma: \mathbb{R}^{n} \rightarrow \mathbb{R}^{n}$ bijeção linear. Então,

$$
A=\pi_{m, n}[B], \text { com } m \geq n \text { e } B \in \mathcal{S}_{m} .
$$

Considere a função $f: \mathbb{R}^{m} \rightarrow \mathbb{R}^{m}$ dada por

$$
f\left(x_{1}, \ldots, x_{m}\right)=\left(\sigma\left(x_{1}, \ldots, x_{n}\right), x_{n+1}, \ldots, x_{m}\right) .
$$

Então, $f$ é bijeção linear. Logo, por hipótese, $f[B] \in \mathcal{S}^{m}$. Além disso,

$$
\sigma[A]=\sigma\left[\pi_{m, n}[B]\right]=\pi_{m, n}[f[B]]
$$

Portanto, $\sigma[A] \in \mathcal{S}_{n}^{p r}$.

$\mathcal{S}^{p r}$ é o-minimal.

(EF5) Sejam $n \geq 1$ e $A \in \mathcal{S}_{n}^{p r}$. Então,

$$
A=\pi_{m, n}\left[A^{\prime}\right], \operatorname{com} m \geq n \text { e } A^{\prime} \in \mathcal{S}_{m}
$$

Por hipótese, existe $N \in \mathbb{N}$ tal que, dado $X \subseteq \mathbb{R}^{m}$ subespaço afim,

$$
A^{\prime} \cap X=A_{1}^{\prime} \cup \ldots \cup A_{N}^{\prime}, \operatorname{com} A_{1}^{\prime}, \ldots, A_{N}^{\prime} \subseteq \mathbb{R}^{m} \text { conexos. }
$$

Além disso, $N$ é o menor natural com a propriedade (OM), para $A^{\prime}$. 
Seja $Y \subseteq \mathbb{R}^{n}$ subespaço afim qualquer. Então,

$$
A \cap Y=A \cap \pi_{m, n}\left[Y \times \mathbb{R}^{m-n}\right]=\pi_{m, n}\left[A^{\prime}\right] \cap \pi_{m, n}\left[Y \times \mathbb{R}^{m-n}\right]=\pi_{m, n}\left[A^{\prime} \cap\left(X \times \mathbb{R}^{m-n}\right)\right]
$$

Uma vez que $Y \times \mathbb{R}^{m-n}$ é subespaço afim de $\mathbb{R}^{m}$, então, existem $A_{1}^{\prime}, \ldots, A_{N}^{\prime}$ conexos tais que

$$
A^{\prime} \cap\left(Y \times \mathbb{R}^{m-n}\right)=A_{1}^{\prime} \cup \ldots \cup A_{N}^{\prime}
$$

Portanto,

$$
A \cap Y=\pi_{m, n}\left[A^{\prime} \cap\left(X \times \mathbb{R}^{m-n}\right)\right]=\pi_{m, n}\left[A_{1}^{\prime} \cup \ldots \cup A_{N}^{\prime}\right]=\pi_{m, n}\left[A_{1}^{\prime}\right] \cup \ldots \cup \pi_{m, n}\left[A_{N}^{\prime}\right]
$$

de sorte que $\pi_{m, n}\left[A_{i}^{\prime}\right] \subseteq \mathbb{R}^{n}$ é conexo, já que $\pi_{m, n}$ é contínua.

Dessa forma, basta tomarmos $\gamma(A) \leq N$.

(EF6) Sejam $n \geq 1$ e $A \in \mathcal{S}_{n}^{p r}$. Então,

$$
A=\pi_{m, n}\left[A^{\prime}\right], \operatorname{com} m \geq n \text { e } A^{\prime} \in \mathcal{S}_{m}
$$

Por hipótese, existem $p \geq m$ e $B \in \mathcal{S}_{p}$ fechado tais que $A^{\prime}=\pi_{p, m}[B]$. Logo,

$$
A=\pi_{m, n}\left[A^{\prime}\right]=\pi_{m, n} \circ \pi_{p, m}[B]=\pi_{p, n}[B] .
$$

Como $B=\imath[B]$, em que $\imath: \mathbb{R}^{p} \rightarrow \mathbb{R}^{p}$ é a identidade, então $B \in \mathcal{S}_{p}^{p r}$ e, assim, segue (EF6). $\underline{\mathcal{S}^{c l} \text { é estrutura fraca. }}$

(EF1) Sejam $A, B \in \mathcal{S}_{n}^{c l}$. Então,

$$
A=A_{0} \cap \bigcap_{i=1}^{p} \bar{A}_{i} \text { e } A=B_{0} \cap \bigcap_{i=1}^{p^{\prime}} \bar{B}_{i}
$$


$\operatorname{com} p, p^{\prime} \geq 0$ e $A_{0}, \ldots, A_{p}, B_{0}, \ldots, B_{p^{\prime}} \in \mathcal{S}^{n}$. Logo,

$$
A \cap B=A_{0} \cap B_{0} \cap \bigcap_{i=1}^{p+p^{\prime}} I_{i}
$$

com

$$
I_{i}=\left\{\begin{array}{l}
\bar{A}_{i}, \quad \text { para } 1 \leq i \leq p \\
\bar{B}_{i}, \quad \text { para } p+1 \leq i \leq p+p^{\prime}
\end{array} .\right.
$$

Por hipótese, $A_{0} \cap B_{0} \in \mathcal{S}_{n}$. Portanto, $A \cap B \in \mathcal{S}_{n}^{c l}$.

(EF2) Seja $A \subseteq \mathbb{R}^{n}$ subconjunto semi-algébrico qualquer. Por hipótese, $A \in \mathcal{S}_{n}$. Por conseguinte, $A \in \mathcal{S}_{n}^{c l}$.

(EF3) Sejam $A \in \mathcal{S}_{n}^{c l}$ e $B \in \mathcal{S}_{m}^{c l}$. Então,

$$
A=A_{0} \cap \bigcap_{i=1}^{p} \bar{A}_{i} \text { e } B=B_{0} \cap \bigcap_{i=1}^{p^{\prime}} \bar{B}_{i}
$$

$\operatorname{com} p, p^{\prime} \geq 0$ e $A_{0}, A_{1}, \ldots, A_{p} \in \mathcal{S}_{n}$ e $B_{0}, B_{1}, \ldots, B_{p^{\prime}} \in \mathcal{S}^{m}$. Logo,

$$
A \times B=\left(A_{0} \cap \bigcap_{i=1}^{p} \bar{A}_{i}\right) \times\left(B_{0} \cap \bigcap_{i=1}^{p^{\prime}} \bar{B}_{i}\right)=\left(A_{0} \times B_{0}\right) \cap\left(\bigcap_{i=1}^{p} \bar{A}_{i} \times \bigcap_{i=1}^{p^{\prime}} \bar{B}_{i}\right) .
$$

Suponha, sem perda de generalidade, que $p^{\prime} \leq p$. Então,

$$
\left(A_{0} \times B_{0}\right) \cap\left(\bigcap_{i=1}^{p} \bar{A}_{i} \times \bigcap_{i=1}^{p^{\prime}} \bar{B}_{i}\right)=\left(A_{0} \times B_{0}\right) \cap \bigcap_{i=1}^{p} I_{i}
$$

com

$$
I_{i}=\left\{\begin{array}{ll}
\bar{A}_{i} \times \bar{B}_{i}=\overline{A_{i} \times B_{i}}, & \text { para } 1 \leq i \leq p^{\prime} \\
\bar{A}_{i} \times \bar{B}_{p^{\prime}}=\overline{A_{i} \times B_{p^{\prime}}}, & \text { para } p^{\prime} \leq i \leq p
\end{array} .\right.
$$

Por hipótese, 


$$
A_{0} \times B_{0} \in \mathcal{S}_{n+m}, A_{i} \times B_{i} \in \mathcal{S}_{n+m}\left(i=1, \ldots, p^{\prime}\right)
$$

e

$$
A_{i} \times B_{p^{\prime}} \in \mathcal{S}_{n+m}\left(i=p^{\prime}, \ldots, p\right)
$$

Portanto, $A \times B \in \mathcal{S}_{n+m}^{c l}$.

(EF4) Sejam $A \in \mathcal{S}_{n}^{c l}$ e $\sigma: \mathbb{R}^{n} \rightarrow \mathbb{R}^{n}$ bijeção linear. Então,

$$
A=A_{0} \cap \bigcap_{i=1}^{p} \bar{A}_{i}, \operatorname{com} A_{0}, \ldots, A_{p} \in \mathcal{S}_{n} .
$$

Logo,

$$
\sigma[A]=\sigma\left[A_{0} \cap \bigcap_{i=1}^{p} \bar{A}_{i}\right] \stackrel{(\sigma \text { é bij. })}{=} \sigma\left[A_{0}\right] \cap \sigma\left[\bigcap_{i=1}^{p} \bar{A}_{i}\right] \stackrel{(\sigma \text { é bij. })}{=} \sigma\left[A_{0}\right] \cap \bigcap_{i=1}^{p} \sigma\left[\bar{A}_{i}\right]
$$

Uma vez que $\sigma$ é homeomorfismo,

$$
\sigma\left[\bar{A}_{i}\right]=\overline{\sigma\left[A_{i}\right]}
$$

Assim,

$$
\sigma[A]=\sigma\left[A_{0}\right] \cap \bigcap_{i=1}^{p} \overline{\sigma\left[A_{i}\right]} .
$$

Por hipótese, $\sigma\left[A_{i}\right] \in \mathcal{S}_{n}$, para $i=0,1, \ldots, p$.

Portanto, $\sigma[A] \in \mathcal{S}_{n}^{c l}$.

$\underline{\mathcal{S}^{c l} \text { é o-minimal. }}$.

(EF5) Antes de mostrarmos que $\mathcal{S}^{c l}$ satisfaz a condição (EF5) propriamente dita, considere o seguinte resultado.

AfirmaÇÃo. Sejam $n \geq 1$ e $D \in \mathcal{S}_{n}$. Então, existe $N \in \mathbb{N}$ tal que, para todo subespaço afim $X \subseteq \mathbb{R}^{n}$, o conjunto $\bar{D} \cap X$ tem no máximo $N$ componentes conexas. 
Prova da Afirmação. Seja $p(\bar{x}, \vec{y}) \in \mathbb{Z}[\bar{x}, \vec{y}]$ com a propriedade: dado qualquer subespaço afim $Z \subseteq \mathbb{R}^{n}$ existem parâmetros $b_{1}, \ldots, b_{m} \in \mathbb{R}$ tais que

$$
Z=\left\{\bar{x} \in \mathbb{R}^{n}: p(\bar{x}, \vec{b})=0\right\}
$$

Seja $E$ o seguinte conjunto semi-algébrico

$$
\left\{(\bar{x}, \vec{y}, R, \epsilon) \in \mathbb{R}^{n+m+2}:|p(\bar{x}, \vec{y})|<\epsilon \wedge \sum_{i=1}^{n} x_{i}^{2} \leq R^{2}\right\}
$$

Visto que $E \in \mathcal{S}_{n+m+2}$, então

$$
E \cap\left(D \times \mathbb{R}^{m+2}\right) \in \mathcal{S}_{n+m+2}
$$

Portanto, por (EF5), existe $N \in \mathbb{N}$ tal que:

(†) para todo $W \subseteq \mathbb{R}^{m+n+2}$ subconjunto afim, $E \cap\left(D \times \mathbb{R}^{m+2}\right) \cap W$ tem no máximo $N$ componentes conexas.

Vamos mostrar que este $N$ vale também para $\bar{D}$.

Assuma, por contradição, que exista $X \subseteq \mathbb{R}^{n}$ afim, tal que

$$
\bar{D} \cap X \text { tem } C_{1}, \ldots, C_{q} \text { componentes conexas, com } q>N \text {. }
$$

Note que, as componentes conexas são subconjuntos fechados (e não vazios) de $\bar{D} \cap X$ e este é fechado de $\mathbb{R}^{n}$, então $C_{1}, \ldots, C_{q}$ são fechados de $\mathbb{R}^{n}$ também. Visto que $\mathbb{R}^{n}$ é normal ${ }^{3}$, então existem $U_{i}$ 's abertos, dois a dois disjuntos, tal que $C_{i} \subseteq U_{i}$. Logo,

$$
\bar{D} \cap X=\bigcup_{i=1}^{q} C_{i} \subseteq \bigcup_{i=1}^{q} U_{i} \text { e } T_{i}=U_{i} \cap \bar{D} \cap X \neq \emptyset
$$

Para cada $i \in\{1, \ldots, q\}$, fixe $\bar{x}_{i} \in T_{i}$.

\footnotetext{
${ }^{3}$ Um espaço topológico $X$ é normal se, e somente se, (para todo $x \in X$ ) o conjunto $\{x\}$ é fechado e para cada par de fechados disjuntos $F$ e $G$ existem abertos $U$ e $V$ disjuntos que contêm $F$ e $G$.
} 
Então, tomando $R_{0}>\max \left\{\left\|\bar{x}_{i}\right\|: \bar{x}_{i} \in T_{i}\right.$ e $\left.i=1, \ldots, q\right\}$, temos que

$$
\text { para todo } i \in\{1, \ldots, q\} \text {, existe } \bar{x} \in T_{i} \text { tal que } \sum_{i=1}^{n} x_{i}^{2}<R_{0}^{2} \text {. }
$$

Denotemos, por $B_{R_{0}}$ e $C$, respectivamente, os conjuntos

$$
\left\{\bar{x} \in \mathbb{R}^{n}: \sum_{i=1}^{n} x_{i}^{2} \leq R_{0}^{2}\right\} \text { e }\left(\mathbb{R}^{n} \backslash \bigcup_{i=1}^{q} U_{i}\right) \cap \bar{D} \cap B_{R_{0}} .
$$

Como $C$ é intersecção de fechados, então também é fechado. Além disso, $C \subseteq B_{R_{0}}, i$. e., é limitado. Assim, $C$ é compacto.

Escolha $\vec{b} \in \mathbb{R}^{m}$ tal que

$$
X=\{\bar{x}: p(\bar{x}, \vec{b})=0\}
$$

Note que $C \cap X=\emptyset$.

Como a função $\bar{x} \mapsto|p(\bar{x}, \vec{b})|$ é contínua em $C$ e este é compacto, então, pelo Teorema de Weierstrass, esta função assume valor mínimo em $C$, digamos $\epsilon_{0}$.

Uma vez que,

$$
\forall \bar{x} \in \mathbb{R}^{n},|p(\bar{x}, \vec{b})| \geq 0 \text { e } C \cap X=\emptyset
$$

então

$$
\forall \bar{x} \in C,|p(\bar{x}, \vec{b})|>0
$$

Assim, $\epsilon_{0}>0$.

Considere o conjunto afim

$$
Y=\mathbb{R}^{n} \times\left\{\left(\vec{b}, R_{0}, \epsilon_{0}\right)\right\}
$$

Então, $S=E \cap\left(D \times \mathbb{R}^{m+2}\right) \cap Y$ tem mais de $N$ componentes conexas.

De fato, note que

$$
S=S_{0} \times\left\{\left(\vec{b}, R_{0}, \epsilon_{0}\right)\right\},
$$


com

$$
S_{0}=\left\{\bar{x} \in D:|p(\bar{x}, \vec{b})|<\epsilon_{0} \wedge \sum_{i=1}^{n} x_{i}^{2} \leq R_{0}^{2}\right\}
$$

$\log ^{4}, S \subseteq\left(\bigcup_{i=1}^{q} U_{i}\right) \times \mathbb{R}^{m+2}$.

Além disso, $S \cap\left(U_{i} \times \mathbb{R}^{m+2}\right) \neq \emptyset$, para $i=1, \ldots, q$.

Com efeito, fixe $i \in\{1, \ldots, q\}$. Então, existe $\bar{x} \in T_{i}$ tal que

$$
\sum_{i=1}^{n} x_{i}^{2}<R_{0}^{2} \text { e } p(\bar{x}, \vec{b})=0\left(\text { pois } T_{1} \subseteq X\right)
$$

Assim, $\bar{x} \in U_{i}$. Logo, como $U_{i}$ é aberto, existe $W_{1} \subseteq U_{i}$ (podemos tomar $W_{1}=U_{i}$ ) aberto tal que $\bar{x} \in W_{1}$. Pela continuidade de $p$, segue que existe uma vizinhança aberta $W_{2}$ de $\bar{x}$ de sorte que,

$$
\forall \bar{w} \in W_{2},|p(\bar{w}, \vec{b})-p(\bar{x}, \vec{b})|=|p(\bar{w}, \vec{b})|<\epsilon_{0}
$$

Considere, agora, a seguinte vizinhança aberta de $\bar{x}$

$$
W=\left\{\bar{w} \in \mathbb{R}^{n}: \sum_{i=1}^{n} w_{i}^{2}<R_{0}^{2}\right\} \cap W_{1} \cap W_{2} .
$$

Então,

(a) $W \subseteq U_{i}$.

(b) $\forall \bar{w} \in W,|p(\bar{w}, \vec{b})|<\epsilon_{0}$.

(c) $W \subseteq B_{R_{0}}$.

\footnotetext{
${ }^{4}$ Seja $(\bar{x}, \vec{y}, t, s) \in S$, então $\bar{x} \in S_{0}, \vec{y}=\vec{b}, t=R_{0}$ e $s=\epsilon_{0} . \operatorname{Logo}, \bar{x} \in D,|p(\bar{x}, \vec{b})|<\epsilon_{0}$ e $\sum_{i=1}^{n} x_{i}^{2} \leq R_{0}^{2}$. De $|p(\bar{x}, \vec{b})|<\epsilon_{0}$, segue que $\bar{x} \notin C$. Desta última, de $\bar{x} \in D$ e de $\sum_{i=1}^{n} x_{i}^{2} \leq R_{0}^{2}$, segue, portanto, que $\bar{x} \in \bigcup_{i=1}^{q} U_{i}$.
} 
Como $\bar{x} \in \bar{D}$, então existe $\bar{w}_{0} \in W$ tal que $\bar{w}_{0} \in D$. Logo, de (b) e (c), concluímos que $\bar{w}_{0} \in S_{0}$. Assim,

$$
\left(\bar{w}_{0}, \vec{b}, R_{0}, \epsilon_{0}\right) \in S
$$

De (a),

$$
\left(\bar{w}_{0}, \vec{b}, R_{0}, \epsilon_{0}\right) \in U_{i} \times \mathbb{R}^{m+2}
$$

Ou seja,

$$
\left(\bar{w}_{0}, \vec{b}, R_{0}, \epsilon_{0}\right) \in S \cap\left(U_{i} \times \mathbb{R}^{m+2}\right) .
$$

Visto que, os $U_{i}$ 's são abertos dois a dois disjuntos, então os $U_{i} \times \mathbb{R}^{m+2}$ 's também o são. Portanto, juntamente com as afirmações

$$
S \cap\left(U_{i} \times \mathbb{R}^{m+2}\right) \neq \emptyset \text { e } S \subseteq\left(\bigcup_{i=1}^{q} U_{i}\right) \times \mathbb{R}^{m+2}
$$

segue que $S$ tem mais de $N$ componentes conexas, o que contradiz $(\dagger)$.

Agora, sejam $n \geq 1$ e $A \in \mathcal{S}_{n}^{c l}$. Então,

$$
A=A_{0} \cap \bigcap_{i=1}^{p} \bar{A}_{i}, \text { com } p \geq 0 \text { e } A_{0}, \ldots, A_{p} \in \mathcal{S}_{n} .
$$

Por (EF6), para $\mathcal{S}_{n}$, temos que

$$
A_{0}=\pi_{m, n}[C], \text { com } m \geq n \text { e } C \in \mathcal{S}_{m} \text { fechado. }
$$

Seja $X \subseteq \mathbb{R}^{n}$ um subespaço afim. Chamemos de $B$ o conjunto

$$
A_{1} \times \ldots \times A_{p} \in \mathcal{S}_{p n}
$$

e

$$
\Delta=\left\{\left(x_{1}, \ldots, x_{(p+1) n}\right) \in \mathbb{R}^{n p+n}:\left(x_{1}, \ldots, x_{n}\right)=\left(x_{n+1}, \ldots, x_{2 n}\right)=\ldots=\left(x_{n p+1}, \ldots, x_{n p+n}\right)\right\}
$$


Denotemos, também, por $Y$, o conjunto $X^{p+1} \cap \Delta$. Assim,

$$
\begin{aligned}
A \cap X & =\pi_{(p+1) n, n}\left[\left(\bar{A}_{1} \times \cdots \times \bar{A}_{p} \times A_{0}\right) \cap\left(X^{p+1} \cap \Delta\right)\right] \\
& =\pi_{(p+1) n, n}\left[\left(\bar{B} \times \pi_{m, n}[C]\right) \cap Y\right] \\
& =\pi_{(p+1) n, n}\left[\pi_{p n+m, p n+n}[\bar{B} \times C] \cap Y\right] \\
& =\pi_{(p+1) n, n} \circ \pi_{p n+m, p n+n}\left[(\bar{B} \times C) \cap\left(Y \times \mathbb{R}^{m-n}\right)\right] \\
& =\pi_{p n+m, n}\left[\overline{B \times C} \cap\left(Y \times \mathbb{R}^{m-n}\right)\right],
\end{aligned}
$$

já que $C$ é fechado.

Como $B \times C \in \mathcal{S}_{p n+m}$, então, pela Afirmação, existe um $N \in \mathbb{N}$ tal que

$$
\overline{B \times C} \cap\left(Y \times \mathbb{R}^{m-n}\right) \text { tem no máximo } N \text { componentes conexas. }
$$

Visto que, o número de componentes conexas de um conjunto não cresce sob projeção, então

$A \cap X=\pi_{p n+m, n}\left[\overline{B \times C} \cap\left(Y \times \mathbb{R}^{m-n}\right)\right]$ tem no máximo $N$ componentes conexas.

(EF6) Sejam $n \geq 1$ e $A \in \mathcal{S}_{n}^{c l}$. Então,

$$
A=A_{0} \cap \bigcap_{i=1}^{p} \bar{A}_{i}, \text { com } p \geq 0 \text { e } A_{0}, \ldots, A_{p} \in \mathcal{S}_{n}
$$

Por hipótese, existem $m \geq n$ e $C \in \mathcal{S}_{m}$ fechado tal que $A_{0}=\pi_{m, n}[C]$.

Seja

$$
B=\mathbb{R}^{n} \cap \bigcap_{i=1}^{p} \bar{A}_{i}
$$

Como $\mathbb{R}^{n} \in \mathcal{S}_{n}$, então $B \in \mathcal{S}_{n}^{c l}$. Além disso, $B$ é fechado. Assim,

$$
A=A_{0} \cap \bigcap_{i=1}^{p} \bar{A}_{i}=\pi_{m, n}[C] \cap B=\pi_{m, n}\left[C \cap\left(B \times \mathbb{R}^{m-n}\right)\right]
$$


Uma vez que $B \times \mathbb{R}^{m-n}$ e $C$ são fechados, então $C \cap\left(B \times \mathbb{R}^{m-n}\right)$ também o é. Além disso,

$$
C \cap\left(B \times \mathbb{R}^{m-n}\right) \in \mathcal{S}_{m}
$$

o que implica

$$
C \cap\left(B \times \mathbb{R}^{m-n}\right) \in \mathcal{S}_{m}^{c l}
$$

e, portanto, segue (EF6).

Notação. Se $f$ é uma função, então $f \in \mathcal{S}$ significa que Dom $(f) \in \mathbb{R}^{n}, \operatorname{Im}(f) \in \mathbb{R}^{m} e$ $G r(f) \in \mathcal{S}_{n+m}$, para $n, m \geq 1$.

As idéias principais usadas nesta próxima definição, assim como nos lemas dispersos no restante desta seção, apareceram originalmente em [3], no qual Charbonnel considera os correspondentes aos $\mathcal{S}_{n}$ 's da Definição 2.1 .3 como álgebras ${ }^{5}$ de coleções de funções da forma $\bar{x} \mapsto p_{1}(\bar{x}) e^{L_{1}(\bar{x})}+\cdots+p_{k}(\bar{x}) e^{L_{k}(\bar{x})}$, em que $p_{1}, \ldots, p_{k} \in \mathbb{R}[\bar{x}]$ e $L_{1}, \ldots, L_{k}: \mathbb{R}^{n} \rightarrow \mathbb{R}$ são funcionais lineares. Todavia, em [21], Wilkie apresenta retificação de algumas demonstrações em [3] que continham erros.

Definição 2.1.10. Seja $\mathcal{S}$ uma pré-estrutura. Definimos:

(i) $\mathcal{S}^{0}=\mathcal{S}$

(ii) $\mathcal{S}^{i+1}=\left(\left(\left(\mathcal{S}^{i}\right)^{u}\right)^{p r}\right)^{c l}$.

(iii) $C h(\mathcal{S})=\bigcup_{i \in \mathbb{N}} \mathcal{S}^{i}$

Alguns textos referem-se à coleção $C h(\mathcal{S})$ como fecho Charbonnel de $\mathcal{S}$.

Como consequência da definição, temos que $\mathcal{S}^{m} \subseteq \mathcal{S}^{k}$, para $m \leq k$.

O corolário ulterior (do Lema 2.1.9) estabelece que o fecho Charbonnel de uma estrutura fraca o-minimal é algo que se aproxima de uma estrutura o-minimal no sentido da Definição 2.0.7.

\footnotetext{
${ }^{5}$ Uma álgebra $A$ sobre um corpo $K$ é um espaço vetorial sobre $K$ equipado com uma operação binária $\circ: A \times A \rightarrow A$ tal que, para $x, y, z \in A$ e $a, b \in K$ arbitrários: $x \circ(y+z)=x \circ y+x \circ z,(x+y) \circ z=x \circ z+y \circ z$ $\mathrm{e}(a x) \circ(b y)=(a b)(x \circ y)$.
} 
Corolário 2.1.11. Se $\mathcal{S}$ é uma estrutura fraca o-minimal, então $C h(\mathcal{S})$ também o é. Além disso, $C h(\mathcal{S})$ é fechada sob intersecção finita, união finita, projeção e fecho topológico.

\section{Demonstração.}

AfirmaÇÃo. Para todo $i \geq 0, \mathcal{S}^{i}$ é uma estrutura fraca o-minimal.

Prova da Afirmação. De fato, para $i=0$, temos que $\mathcal{S}^{0}=\mathcal{S}$ e, portanto, por hipótese, segue o resultado.

Suponha que $\mathcal{S}^{i}$ seja estrutura fraca o-minimal. Logo, aplicando o Lema 2.1.9, sucessivamente para $\left(\mathcal{S}^{i}\right),\left(\mathcal{S}^{i}\right)^{u}$ e $\left(\left(\mathcal{S}^{i}\right)^{u}\right)^{p r}$, segue que $\left(\left(\left(\mathcal{S}^{i}\right)^{u}\right)^{p r}\right)^{c l}=\mathcal{S}^{i+1}$ é estrutura fraca o-minimal.

$C h(\mathcal{S})$ é estrutura fraca o-minimal.

$(\mathrm{EF} 1)$ Sejam $A, B \in C h(\mathcal{S})_{n}$. Então, $A \in \mathcal{S}_{n}^{m}$ e $B \in \mathcal{S}_{n}^{k}$.

Suponha, sem perda de generalidade, que $m \leq k$.

Logo, $\mathcal{S}_{n}^{m} \subseteq \mathcal{S}_{n}^{k}$. Assim, $A \in \mathcal{S}_{n}^{k}$ e, portanto, pela Afirmação, $A \cap B \in \mathcal{S}_{n}^{k} \subseteq C h(\mathcal{S})_{n}$.

(EF2) Seja $A \subseteq \mathbb{R}^{n}$, com $1 \leq n$, semi-algébrico qualquer. Então, por hipótese, $A \in \mathcal{S}$. Portanto, $A \in C h(\mathcal{S})$.

(EF3) Sejam $A \in C h(\mathcal{S})_{n}$ e $B \in C h(\mathcal{S})_{m}, \operatorname{com} n, m \geq 1$. Então, $A \in \mathcal{S}_{n}^{i}$ e $B \in \mathcal{S}_{m}^{j}$, para $i, j \geq 0$.

Suponha $i \leq j$.

Logo, $A \in \mathcal{S}_{n}^{j}$. Portanto, $A \times B \in \mathcal{S}_{m+n}^{j}$ e, assim $A \times B \in C h(\mathcal{S})_{m+n}$.

(EF4) Sejam $A \in C h(\mathcal{S})$ e $\sigma: \mathbb{R}^{n} \rightarrow \mathbb{R}^{n}$ bijeção linear. Então, existe $i \in \mathbb{N}$ tal que $A \in \mathcal{S}_{n}^{i}$ e, portanto, pela Afirmação, $\sigma[A] \in \mathcal{S}_{n}^{i} \subseteq C h(\mathcal{S})_{n}$.

(EF5) Considere $A \in C h(\mathcal{S})$. Então, $A \in \mathcal{S}^{i}$. Logo, pela Afirmação, $\gamma(A)<\infty$.

(EF6) Seja $A \in C h(\mathcal{S})$. Então, $A \in \mathcal{S}_{n}^{i}$. Logo, pela Afirmação, existem $m \geq n$ e $B \in \mathcal{S}_{m}^{i}$ (e, portanto, $B \in C h(\mathcal{S})_{m}$ ) fechado tal que $A=\pi_{m, n}[B]$.

$\underline{C h(\mathcal{S}) \text { é fechada sob intersecção finita. }}$ 
Vejamos que para todo $k \geq 2, \bigcap_{i=1}^{k} A_{i} \in C h(\mathcal{S})_{n}$, sendo que $A_{1}, \ldots, A_{k} \in C h(\mathcal{S})_{n}$.

Com efeito, para $k=2$, temos, por (EF1), que $A_{1} \cap A_{2} \in C h(\mathcal{S})_{n}$.

Suponha que $\bigcap_{i=1}^{k} A_{i} \in C h(\mathcal{S})_{n}$, para $A_{1}, \ldots, A_{k} \in C h(\mathcal{S})_{n}$. Logo, dado $A_{k+1} \in C h(\mathcal{S})_{n}$

$$
\bigcap_{i=1}^{k+1} A_{i}=A_{k+1} \cap \bigcap_{i=1}^{k} A_{i} \in C h(\mathcal{S})_{n}
$$

pela hipótese de indução e por (EF1).

$\underline{C h(\mathcal{S}) \text { é fechada sob união finita. }}$

Vejamos que para todo $k \geq 2, \bigcup_{i=1}^{k} A_{i} \in C h(\mathcal{S})_{n}$, em que $A_{1}, \ldots, A_{k} \in C h(\mathcal{S})_{n}$.

Sejam $A_{1}, \ldots, A_{k} \in C h(\mathcal{S})_{n}, \operatorname{com} k \geq 2$. Então,

$$
A_{1} \in \mathcal{S}_{n}^{m_{1}}, \ldots, A_{k} \in \mathcal{S}_{n}^{m_{k}}
$$

Tomemos $m=\max \left\{m_{i}: i=1, \ldots, k\right\}$. Logo, $A_{i} \in \mathcal{S}^{m}$, para todo $i$. Portanto,

$$
\bigcup_{i=1}^{k} A_{i} \in\left(\mathcal{S}^{m}\right)_{n}^{u}
$$

Consequentemente,

$$
\bigcup_{i=1}^{k} A_{i} \in\left(\left(\left(\mathcal{S}^{m}\right)^{u}\right)^{p r}\right)_{n}^{c l}=\mathcal{S}_{n}^{m+1}
$$

E, assim,

$$
\bigcup_{i=1}^{k} A_{i} \in C h(\mathcal{S})_{n}
$$

$\underline{C h(\mathcal{S}) \text { é fechada sob projeção. }}$ 
Sejam $A \in C h(\mathcal{S})_{n}$ e $k \leq n$. Então, $A \in \mathcal{S}_{n}^{m}$, para algum $m \geq 0$.

Logo, $A \in\left(\mathcal{S}^{m}\right)_{n}^{u}$. Por conseguinte,

$$
\pi_{n, k}[A] \in\left(\left(\mathcal{S}^{m}\right)^{u}\right)_{k}^{p r}
$$

Pela definição de pré-estrutura $\mathcal{S}^{c l}$, segue que

$$
\pi_{n, k}[A] \in\left(\left(\left(\mathcal{S}^{m}\right)^{u}\right)^{p r}\right)_{k}^{c l}=\mathcal{S}_{k}^{m+1}
$$

Portanto,

$$
\pi_{n, k}[A] \in C h(\mathcal{S})_{k}
$$

$C h(\mathcal{S})$ é fechada sob fecho topológico.

Seja $A \in C h(\mathcal{S})_{n}$. Então, para algum $m \geq 0, A \in \mathcal{S}_{n}^{m}$.

Logo,

$$
A \in\left(\left(\mathcal{S}^{m}\right)^{u}\right)_{n}^{p r}
$$

Segue, pela definição de pré-estrutura $\mathcal{S}^{c l}$, que

$$
\bar{A}=\mathbb{R}^{n} \cap \bar{A} \in\left(\left(\left(\mathcal{S}^{m}\right)^{u}\right)^{p r}\right)_{n}^{c l}=\mathcal{S}_{n}^{m+1}
$$

E, portanto, $\bar{A} \in C h(\mathcal{S})_{n}$

Aviso. Daqui em diante, faremos referência à $C h(\mathcal{S})$, com a suposição de que $\mathcal{S}$ é estrutura fraca o-minimal.

De acordo com a Definição 2.1.3 e com o Corolário 2.1.11, para determinarmos se um dado subconjunto $A \subseteq \mathbb{R}^{n}$ pertence a $C h(\mathcal{S})$ basta que escrevamos $A$ como uma sequência de operações de intersecção, união, projeção e fecho topológico efetuadas sobre conjuntos de $C h(\mathcal{S})$. Obviamente, quando o conjunto $A$ apresentado já está neste molde, temos imediatamente que $A \in C h(\mathcal{S})$. Contudo, com grande frequência, lidaremos com $A$ descrito por uma fórmula 
cuja interpretação em termos destas operações conjuntistas é pouco evidente. Torna-se, portanto, um tanto conveniente especificarmos as fórmulas que descrevem os elementos do fecho Charbonnel.

Definição 2.1.12. Seja $L^{\prime}$ uma linguagem que expande $L^{6}$. Definimos uma $C h$-fórmula do seguinte modo:

(i) Todas as fórmulas atômicas de $L^{\prime}$ são $C h$-fórmulas.

(ii) Se $\psi$ e $\phi$ são $C h$-fórmulas e $x$ é uma variável, então $\psi \wedge \phi, \psi \vee \phi$ e $\exists x \phi$ são $C h$-fórmulas.

(iii) Se $\phi$ é uma $C h$-fórmula, então $\bar{\phi}$ é uma $C h$-fórmula, em que

$$
\overline{\phi(\bar{x})} \stackrel{\text { def }}{=} \forall t>0 \exists \bar{u}\left(\phi(\bar{u}) \wedge \sum_{i=1}^{n}\left(u_{i}-x_{i}\right)^{2}<t^{2}\right) .
$$

(iv) Nada mais é uma $C h$-fórmula.

Note que todas as $C h$-fórmulas são $L^{\prime}$-fórmulas. É claro que o inverso não é, em geral, válido.

Decorre do Corolário 2.1.11 e da Definição 2.1.12, caracterizações equipolentes dos elementos de $C h(\mathcal{S})$,

Corolário 2.1.13. As seguintes afirmações são equivalentes:

1. $A \in C h(\mathcal{S})$.

2a. A é igual a uma expressão finita de conjuntos em $\mathcal{S}$ e de operações de intersecção, união, projeção e fecho topológico efetuadas sobre tais conjuntos.

2b. A é definido por uma Ch-fórmula na estrutura $\langle\overline{\mathbb{R}},\{X: X \in \mathcal{S}\}\rangle$.

3a. A é igual a uma expressão finita de conjuntos em $C h(\mathcal{S})$ e de operações de intersecção, união, projeção e fecho topológico efetuadas sobre tais conjuntos.

\footnotetext{
${ }^{6} i$. e., $L \subseteq L^{\prime}$, sendo que $L$ é a linguagem dos anéis ordenados.
} 
3b. A é definido por uma Ch-fórmula na estrutura $\langle\overline{\mathbb{R}},\{Y: Y \in C h(\mathcal{S})\}\rangle$.

Demonstração.

$(1) \Rightarrow(2 \mathrm{a})$

Segue diretamente da definição de $C h(\mathcal{S})$.

$(2 \mathrm{a}) \Rightarrow(3 \mathrm{a})$

Como $\mathcal{S} \subseteq C h(\mathcal{S})$, então segue a afirmação.

$(3 \mathrm{a}) \Rightarrow(1)$

Seja $A$ conforme $(3 \mathrm{a})$. Como $C h(\mathcal{S})$ é fechado sob as operações de intersecção finita, união finita, projeção e fecho topológico, então $A \in C h(\mathcal{S})$.

Agora, considere $L^{\prime}=L \cup\left\{P_{X}: X \in \mathcal{S}_{n}, n \geq 1\right\}$, em que $P_{X}$ é um símbolo relacional $n$-ário e $P_{X}(\bar{x})$ é interpretado em $\langle\overline{\mathbb{R}},\{X: X \in \mathcal{S}\}\rangle$ como $\bar{x} \in X\left(\in \mathcal{S}_{n}\right)$.

$(2 \mathrm{a}) \Rightarrow(2 \mathrm{~b})$

Seja $A$ uma coleção conforme (2a). Mostraremos que $A$ é uma coleção conforme (2b), por indução na complexidade de construção de $A$. De fato, se $A$ é um conjunto de $\mathcal{S}$ ( $\Leftrightarrow A$ tem complexidade 1), então $A=\left\{\bar{x}:\langle\overline{\mathbb{R}},\{X: X \in \mathcal{S}\}\rangle \models P_{A}(\bar{x})\right\}$.

Suponha, então, que $A$ tenha complexidade $k$ e assuma que o resultado seja válido para coleções (conforme (2a)) de complexidade maior ou igual a 1 e menor que $k$. Temos, assim, alguns casos a serem considerados:

(i) $A=A_{1} \cap A_{2}$, em que $A_{1}$ tem complexidade menor que $k$ e $A_{2} \in \mathcal{S}$.

Então, pela hipótese de indução,

$$
A_{1}=\left\{\bar{x}:\langle\overline{\mathbb{R}},\{X: X \in \mathcal{S}\}\rangle \mid=\phi_{A_{1}}(\bar{x})\right\}
$$

sendo que $\phi_{A_{1}}$ é uma $C h$-fórmula, e

$$
A_{2}=\left\{\bar{x}:\langle\overline{\mathbb{R}},\{X: X \in \mathcal{S}\}\rangle \mid=P_{A_{2}}(\bar{x})\right\}
$$


Portanto, $A=A_{1} \cap A_{2}$ é igual a

$$
\left\{\bar{x}:\langle\overline{\mathbb{R}},\{X: X \in \mathcal{S}\}\rangle \models \phi_{A_{1}}(\bar{x}) \wedge P_{A_{2}}(\bar{x})\right\} .
$$

(ii) $A=A_{1} \cup A_{2}$, em que $A_{1}$ tem complexidade menor que $k$ e $A_{2} \in \mathcal{S}$.

Então, pela hipótese de indução, $A_{1}$ é definível, em $\langle\overline{\mathbb{R}},\{X: X \in \mathcal{S}\}\rangle$, por uma $C h$ fórmula $\phi_{A_{1}}(\bar{x})$ e $A_{2}$ por $P_{A_{2}}(\bar{x})$. Assim, $A=A_{1} \cap A_{2}$ é definível, em $\langle\overline{\mathbb{R}},\{X: X \in \mathcal{S}\}\rangle$, pela Ch-fórmula

$$
\phi_{A_{1}}(\bar{x}) \vee P_{A_{2}}(\bar{x})
$$

(iii) $A=\pi_{m, n}\left[A_{1}\right]$, em que $A_{1}$ tem complexidade menor que $k$.

Então, pela hipótese de indução, $A_{1}$ é definível, em $\langle\overline{\mathbb{R}},\{X: X \in \mathcal{S}\}\rangle$, por uma $C h$ fórmula $\phi_{A_{1}}(\bar{x})$. Portanto, $A=\pi_{m, n}\left[A_{1}\right]$ é descrito pela seguinte $C h$-fórmula

$$
\exists v_{n+1} \cdots \exists v_{m} \phi_{A}\left(\bar{x}, v_{n+1}, \ldots, v_{m}\right) .
$$

(iv) $A=\overline{A_{1}}$, em que $A_{1}$ tem complexidade menor que $k$.

Então, pela hipótese de indução, $A_{1}$ é definível, em $\langle\overline{\mathbb{R}},\{X: X \in \mathcal{S}\}\rangle$, por uma $C h$ fórmula $\phi_{A_{1}}(\bar{x})$. Logo, $A=\overline{A_{1}}$ é dado por

$$
\left\{\bar{x}:\langle\overline{\mathbb{R}},\{X: X \in \mathcal{S}\}\rangle \models \overline{\phi_{A_{1}}(\bar{x})}\right\}
$$

$(2 \mathrm{a}) \Leftarrow(2 \mathrm{~b})$

É semelhante ao caso $(2 \mathrm{a}) \Rightarrow(2 \mathrm{~b})$, mas a indução é na complexidade das $C h$-fórmulas.

Idêntico ao caso anterior, apenas trocando $\mathcal{S}$ por $C h(\mathcal{S})$.

Ao longo deste capítulo, usaremos a implicação $(3 b) \Rightarrow(1)$ deste Corolário 2.1.13, amiúde, de forma implícita. 
A seguir, exploraremos mais algumas boas propriedades de $C h(\mathcal{S})$.

Lema 2.1.14. Todos os conjuntos em $C h(\mathcal{S})$ são Lebesgue mensuráveis.

Demonstração. Seja $A \in C h(\mathcal{S})_{n}$, para $n \geq 1$ arbitrário. Então, por (EF6), existem $m \geq n$ e $B \in C h(\mathcal{S})_{m}$ fechado tal que

$$
A=\pi_{m, n}[B]=\pi_{m, n}\left[B \cap \mathbb{R}^{m}\right]=\pi_{m, n}\left[B \cap \bigcup_{i=1}^{\infty}[-i, i]^{m}\right]=\bigcup_{i=1}^{\infty} \pi_{m, n}\left[B \cap[-i, i]^{m}\right]
$$

ou seja, $A$ é união enumerável de compactos, logo é união enumerável de fechados. Como fechados são mensuráveis e união enumerável de mensuráveis é mensurável, então $A$ é mensurável.

Denotemos por $\mu_{n}$ a medida de Lebesgue em $\mathbb{R}^{n}$. Dizemos que $A \subseteq \mathbb{R}^{n}$ é nulo ou de medida nula se $\mu_{n}(A)=0$.

Lema 2.1.15 (Teorema de Fubini para conjuntos de medida positiva). Suponha $A \subseteq \mathbb{R}^{n+1}$ mensurável. Então, são equivalentes:

(a) $\mu_{n+1}(A)>0$.

(b) $\mu_{n}(B)>0$, com $B=\left\{\bar{x} \in \mathbb{R}^{n}: \mu_{1}\left(A_{\bar{x}}\right)>0\right\}$.

Demonstração. Ver [7] e [17], pág. 53.

A demonstração do resultado subsequente é creditada exclusivamente a Maxwell, em [16].

Lema 2.1.16. Seja $A \in C h(\mathcal{S})_{n}$, com $n \geq 1$. Suponha que A não tenha ponto interior. Então, A é $\mu_{n}$-nulo.

Demonstração. Como vimos na demonstração do Lema 2.1.14, um subconjunto $A \in C h(\mathcal{S})_{n}$, $n \geq 1$, é união enumerável de compactos de $\mathbb{R}^{n}$ e, portanto, união enumerável de fechados. 
Também, como a união enumerável de conjuntos $\mu_{n}$-nulos é um $\mu_{n}$-nulo, então basta mostrarmos o resultado em questão para $A$ fechado. Isto será feito por indução em $n$. Para $n=1$, temos que, dado $A \in C h(\mathcal{S})_{1}$ fechado e sem ponto interior, existe $N \in \mathbb{N}$, por (EF5), tal que para todo $X \subseteq \mathbb{R}$ afim, $A \cap X$ tem no máximo $N$ componentes conexas. Tomando $X=\mathbb{R}$, temos que

$A=A \cap \mathbb{R}$ tem $I_{1}, \ldots, I_{m}$ componentes conexas e, portanto, intervalos fechados, com $m \leq N$.

Como $\AA=\emptyset$, então os intervalos $I_{1}, \ldots, I_{m}$ são degenerados ${ }^{7}$ e, $\operatorname{assim}|A|=m \leq N$. Além disso, para cada $i=1, \ldots, m, \mu_{1}\left(I_{i}\right)=0$. Portanto, $\mu_{1}(A)=0$.

Suponha que o resultado valha para $n$. Suponha, por absurdo, que exista $A \in C h(\mathcal{S})_{n+1}$ fechado, sem ponto interior e que não seja $\mu_{n+1}$-nulo.

Por (EF5), existe $N \in \mathbb{N}$ tal que para todo $X \subseteq \mathbb{R}^{n+1}$ afim, $A \cap X$ tem no máximo $N$ componentes conexas. Definimos, para cada par $s \in \mathbb{N}^{+}$e $l \in \mathbb{Z}$, o conjunto

$$
C_{s, l}=\left\{\bar{x} \in \mathbb{R}^{n}: \exists t\left(t \in\left[\frac{l}{s}, \frac{l+1}{s}\right] \wedge \bigwedge_{i=0}^{N}\left(\left(\bar{x}, t+\frac{2 i}{s}\right) \in A\right)\right)\right\} .
$$

Este é definido por uma $C h(\mathcal{S})$-fórmula em $\langle\overline{\mathbb{R}},\{X: X \in \mathcal{S}\}\rangle$. Portanto, $C_{s, l} \in C h(\mathcal{S})$, para quaisquer $s \in \mathbb{N}^{+}$e $l \in \mathbb{Z}$.

Seja $C=\bigcup_{s \in \mathbb{N}+} \bigcup_{l \in \mathbb{Z}} C_{s, l}$.

AFIRMAÇÃO.

(a) $\bar{x} \in C \Leftrightarrow A_{\bar{x}}$ contém um intervalo aberto.

(b) $\bar{x} \notin C \Leftrightarrow A_{\bar{x}}$ contém no máximo $N$ pontos.

Prova da Afirmação.

\footnotetext{
${ }^{7}$ ou seja, são intervalos da forma $[a, a]$, com $a \in \mathbb{R}$.
} 
(a) $(\Rightarrow)$ Seja $\bar{x} \in C$. Então, existem $s \in \mathbb{N}^{+}$e $l \in \mathbb{Z}$ tal que $\bar{x} \in C_{s, l}$. Por definição,

$$
(\bar{x}, t), \ldots,\left(\bar{x}, t+\frac{2 N}{s}\right) \in A
$$

para algum $t \in\left[\frac{l}{s}, \frac{l+1}{s}\right]$.

Suponha que $A_{\bar{x}}$ não contenha um intervalo. Então, para cada $i=0, \ldots, N-1$,

$$
\text { existe } y_{i} \text { tal que } t+\frac{2 i}{s}<y_{i}<t+\frac{2(i+1)}{s} \text { e } y_{i} \notin A_{\bar{x}} \text {. }
$$

Logo,

$$
\left.A_{\bar{x}} \subseteq\right]-\infty, y_{0}[\cup] y_{0}, y_{1}[\cup \cdots \cup] y_{N-2}, y_{N-1}[\cup] y_{N-1}, \infty[
$$

$\mathrm{e}$

$$
\left.t \in A_{0}=\right]-\infty, y_{0}\left[, t+\frac{2}{s} \in A_{1}=\right] y_{0}, y_{1}\left[, \ldots, t+\frac{2 N}{s} \in A_{N}=\right] y_{N-1}, \infty[
$$

Tomando $X=\{\bar{x}\} \times \mathbb{R} \subseteq \mathbb{R}^{n+1}$ afim, temos que

$$
A \cap X=\{\bar{x}\} \times A_{\bar{x}} \subseteq\{\bar{x}\} \times A_{0} \cup \cdots \cup\{\bar{x}\} \times A_{N} .
$$

Assim, $A \cap X$ tem no mínimo $N+1$ componentes conexas. $(\Rightarrow \Leftarrow)$

$(\Leftarrow)$ Seja $] a, b\left[\subseteq A_{\bar{x}}\right.$. Fixe $\left.t \in\right] a, b[$. Então,

$$
\text { existe } \epsilon>0 \text { tal que }] t-\epsilon, t+\epsilon[\subseteq] a, b[\text {. }
$$

Tome $s>0$ de modo que $2 N<\epsilon s$. Logo,

$$
t<t+\frac{2}{s}<t+\frac{4}{s}<\cdots<t+\frac{2 N}{s}<t+\epsilon
$$

e, assim

$$
(\bar{x}, t), \ldots,\left(\bar{x}, t+\frac{2 N}{s}\right) \in A
$$

Resta mostrar que existe $l \in \mathbb{Z}$ de sorte que $t \in\left[\frac{l}{s}, \frac{l+1}{s}\right]$. 
Se $t=0$, então $0 \in\left[0, \frac{1}{s}\right]$.

Se $t>0$, então $t s>0$. Seja

$$
Z_{1}=\{m \in \mathbb{Z}: m \geq t s\}
$$

Pela propriedade arquimediana, $Z_{1} \neq \emptyset$. Logo, pela boa ordem de $\mathbb{N}, Z_{1}$ tem mínimo $m_{0}$. Então, $t s>m_{0}-1$. Por conseguinte,

$$
m_{0}-1<t s \leq m_{0}
$$

Logo,

$$
\frac{m_{0}-1}{s}<t \leq \frac{m_{0}}{s}
$$

ou seja

$$
t \in\left[\frac{m_{0}-1}{s}, \frac{m_{0}}{s}\right]
$$

$\mathrm{O}$ caso $t<0$ é similar ao anterior, basta considerar $Z_{2}=\{m \in \mathbb{Z}: m \geq-t s\}$.

Portanto, $\bar{x} \in C_{s, m_{0}}$.

(b) $(\Rightarrow)$ Suponha $\bar{x} \in \mathbb{R}^{n} \backslash C$. Então, $A_{\bar{x}}$ não pode conter um intervalo.

Agora, suponha, por absurdo, que $A_{\bar{x}}$ tenha $m>N$ pontos. Logo, tomando pontos entre cada par $t_{i}$ e $t_{j}$, para $i, j=1, \ldots, m$, e usando o mesmo argumento que em $(\mathrm{a})(\Rightarrow)$, chegamos que $A \cap X, \operatorname{com} X=\{\bar{x}\} \times \mathbb{R}$, teria mais de $N$ componentes conexas. $(\Rightarrow \Leftarrow)$

$(\Leftarrow)$ Se $A_{\bar{x}}$ contém no máximo $N$ pontos, então $A_{\bar{x}}$ não contém um intervalo. Portanto, por $(\mathrm{a}), \bar{x} \notin C$.

Por suposição, $\mu_{n+1}(A)>0$. Logo, pelo Teorema de Fubini, $\mu_{n}(B)>0$, de sorte que

$$
B=\left\{\bar{x} \in \mathbb{R}^{n}: \mu_{1}\left(A_{\bar{x}}\right)>0\right\}
$$


Uma vez que $B \subseteq C^{8}$

$$
0<\mu_{n}(B) \leq \mu_{n}(C) \leq \sum_{s \in \mathbb{N}^{+}} \sum_{l \in \mathbb{Z}} \mu_{n}\left(C_{s, l}\right)
$$

Assim, existem $r \in \mathbb{N}^{+}, k \in \mathbb{Z}$ tais que $\mu_{n}\left(C_{r, k}\right)>0$. (*)

Também, $C_{r, k} \in C h(\mathcal{S})_{n}$. Além disso, $C_{r, k}$ é fechado. $(* *)$

Com efeito, dado $\bar{y} \in \bar{C}_{r, k}$ temos que existe uma sequência $\left(\bar{y}_{i}\right)_{i} \in C_{r, k}$ tal que $\bar{y}_{i} \rightarrow \bar{y}$.

Por definição, para cada $i$,

$$
\text { existe } t_{i} \in\left[\frac{k}{r}, \frac{k+1}{r}\right] \text { tal que }\left(\bar{y}_{i}, t_{i}\right), \ldots,\left(\bar{y}_{i}, t_{i}+\frac{2 N}{r}\right) \in A \text {. }
$$

Como $\left(t_{i}\right)_{i}$ é uma sequência limitada de reais, então, por Bolzano-Weierstrass, $\left(t_{i}\right)_{i}$ possui subsequência $\left(t_{i_{j}}\right)_{j}$ convergente, $i$. e., $\lim _{j \rightarrow+\infty} t_{i_{j}}=t \in \mathbb{R}$. Mas,

$$
\left(t_{i_{j}}\right)_{j} \in\left[\frac{k}{r}, \frac{k+1}{r}\right] \Rightarrow t \in\left[\frac{k}{r}, \frac{k+1}{r}\right]
$$

Também, como $t \mapsto t+\frac{2 m}{r}$ é contínua, então

$$
t_{i_{j}}+\frac{2}{r} \rightarrow t+\frac{2}{r}, \ldots, t_{i_{j}}+\frac{2 N}{r} \rightarrow t+\frac{2 N}{r}
$$

Assim,

$$
\left(\bar{y}_{i_{j}}, t_{i_{j}}\right) \rightarrow(\bar{y}, t), \ldots,\left(\bar{y}_{i_{j}}, t_{i_{j}}+\frac{2 N}{r}\right) \rightarrow\left(\bar{y}, t+\frac{2 N}{r}\right) .
$$

Logo,

$$
(\bar{y}, t), \ldots,\left(\bar{y}, t+\frac{2 N}{r}\right) \in A,
$$

${ }^{8}$ Seja $\bar{x} \in B$. Então, $\mu_{1}\left(A_{\bar{x}}\right)>0$. Como $\{\bar{x}\} \times A_{\bar{x}}=A \cap(\{\bar{x}\} \times \mathbb{R})$ contém $m \leq N$ componentes conexas, então $A_{\bar{x}}$ é dado por $A_{\bar{x}}=I_{1} \cup \cdots \cup I_{m}$, de modo que os intervalos $I_{i}^{\prime} s$ são dois a dois disjuntos. Assim, $\mu_{1}\left(A_{\bar{x}}\right)=\sum_{i=1}^{m} \mu_{1}\left(I_{i}\right)=\sum_{i=1}^{m}\left|I_{i}\right|>0 \Leftrightarrow\left|I_{j}\right|>0$, para algum $j \Leftrightarrow I_{j}$ é não degenerado. Portanto, $A_{\bar{x}}$ contém um intervalo aberto e, pela Afirmação, $\bar{x} \in C$. 
uma vez que $A$ é fechado.

Portanto, $\bar{y} \in C_{r, k}$.

Consequentemente, por $(*),(* *)$ e pela hipótese de indução, $C_{r, k}$ tem ponto interior. Então, existe um cubo aberto $B_{0} \subseteq C_{r, k}$.

Fixe $\bar{x} \in B_{0}$. Então, existe $t \in\left[\frac{k}{r}, \frac{k+1}{r}\right]$ tal que

$$
(\bar{x}, t), \ldots,\left(\bar{x}, t+\frac{2 N}{r}\right) \in A
$$

Note que, se para todo $i \in\{1, \ldots, N\}$, tivéssemos

$$
\left[t+\frac{2 i-2}{r}, t+\frac{2 i}{r}\right] \nsubseteq A_{\bar{x}}
$$

então, para cada $i$, existiria $\left.x_{i} \in\right] t+\frac{2 i-2}{r}, t+\frac{2 i}{r}\left[\backslash A_{\bar{x}}\right.$. E, assim

$$
\left.A_{\bar{x}} \subseteq\right]-\infty, x_{1}[\cup] x_{1}, x_{2}[\cup \cdots \cup] x_{N},+\infty[,
$$

de sorte que

$$
t \in]-\infty, x_{1}\left[, \ldots, t+\frac{2 N}{r} \in\right] x_{N},+\infty[
$$

Logo,

$$
A \cap(\{\bar{x}\} \times \mathbb{R})=\{\bar{x}\} \times A_{\bar{x}} \subseteq(\{\bar{x}\} \times]-\infty, x_{1}[) \cup \cdots \cup(\{\bar{x}\} \times] x_{N},+\infty[),
$$

i. e.,

$$
A \cap(\{\bar{x}\} \times \mathbb{R}) \text { teria no mínimo } N+1 \text { componentes conexas. }(\Rightarrow \Leftarrow)
$$

Portanto, para todo $\bar{x} \in B_{0}$, existem $t \in\left[\frac{k}{r}, \frac{k+1}{r}\right]$ e $i \in\{1, \ldots, N\}$ tais que

$$
\left[t+\frac{2 i-2}{r}, t+\frac{2 i}{r}\right] \subseteq A_{\bar{x}}
$$


Como consequência imediata, temos

$$
\text { para todo } \bar{x} \in B_{0} \text {, existe um } i \in\{1, \ldots, N\} \text { tal que }\left[\frac{k+2 i-1}{r}, \frac{k+2 i}{r}\right] \subseteq A_{\bar{x}} \text {. }
$$

Considere $\left.I_{i}=\right] \frac{k+2 i-1}{r}, \frac{k+2 i}{r}[$, para $i=1, \ldots, N$.

Seja $B_{0} \times I_{1} \subseteq \mathbb{R}^{n+1}$ aberto. Uma vez que $\stackrel{\AA}{A}=\emptyset$, então

$$
\text { existe }(\bar{x}, a) \in B_{0} \times I_{1} \text { tal que }(\bar{x}, a) \notin A \text {. }
$$

Como $\mathbb{R}^{n+1} \backslash A$ é aberto, então existe aberto

$$
B_{1}^{(1)} \times I_{1}^{(1)} \subseteq \mathbb{R}^{n+1} \text { tal que }(\bar{x}, a) \in B_{1}^{(1)} \times I_{1}^{(1)} \subseteq \mathbb{R}^{n+1} \backslash A
$$

De $(\bar{x}, a) \in B_{0} \times I_{1}$, segue que

$$
\text { existe aberto } B_{1}^{(2)} \times I_{1}^{(2)} \text { tal que }(\bar{x}, a) \in B_{1}^{(2)} \times I_{1}^{(2)} \subseteq B_{0} \times I_{1} \text {. }
$$

Tome $B_{1}=B_{1}^{(1)} \cap B_{1}^{(2)}$. Então, $B_{1} \subseteq B_{0}$, com $B_{1} \neq \emptyset$ (pois, $\bar{x} \in B_{1}$ ), e, para todo $\bar{y} \in B_{1}$,

$$
(\bar{y}, a) \in B_{1} \times I_{1}^{(1)} \text {, o que implica }(\bar{y}, a) \notin A .
$$

Seja $B_{1} \times I_{2} \subseteq \mathbb{R}^{n+1}$ aberto. Então, de $\AA=\emptyset$, temos que existe $(\bar{x}, a) \in\left(B_{1} \times I_{2}\right) \backslash A$. Consequentemente, existem $B_{2}^{(1)} \times I_{2}^{(1)}$ e $B_{2}^{(2)} \times I_{2}^{(2)}$ tal que

$$
(\bar{x}, a) \in B_{2}^{(1)} \times I_{2}^{(1)} \subseteq \mathbb{R}^{n} \backslash A \text { e }(\bar{x}, a) \in B_{2}^{(2)} \times I_{2}^{(2)} \subseteq B_{1} \times I_{2}
$$

Tome $B_{2}=B_{2}^{(1)} \cap B_{2}^{(2)}$. Logo, $\emptyset \neq B_{2} \subseteq B_{1}$. Além disso, para todo $\bar{y} \in B_{2}$, segue que

$$
(\bar{y}, a) \in B_{2} \times I_{2}^{(1)} \text {, ou seja, }(\bar{y}, a) \notin A \text {. }
$$

Repetindo este argumento até $i=N$, chegamos que existem $B_{N} \subseteq B_{N-1} \subseteq \cdots \subseteq B_{0}$ abertos não vazios e

para todo $\bar{x} \in B_{N}$ e todo $i \in\{1, \ldots, N\}$ existe $a_{i} \in I_{i}$ tal que $\left(\bar{x}, a_{i}\right) \notin A$, 
i. e.,

$$
\text { para todo } \bar{x} \in B_{N} \text { e todo } i \in\{1, \ldots, N\},\left[\frac{k+2 i-1}{r}, \frac{k+2 i}{r}\right] \nsubseteq A_{\bar{x}} \text {. }
$$

Logo, de $(\dagger)$, concluímos que $B_{N} \cap B_{0}=\emptyset$. Contradição, pois $B_{N} \subseteq B_{0}$ e $B_{N} \neq \emptyset$.

Bem como a prova do lema precedente, a demonstração deste resultado propínquo também se deve unicamente a Maxwell, em [16].

Lema 2.1.17. Suponha que $A \in C h(\mathcal{S})_{n}, \operatorname{com} n \geq 1$, não tenha ponto interior. Então, $\bar{A}$ ñ̃o tem ponto interior.

Demonstração. Vamos proceder por indução em $n$.

Seja $n=1$. Suponha $A \in C h(\mathcal{S})_{1}$ sem ponto interior. Por $(\mathrm{EF} 5)$, existe $N \in \mathbb{N}$ tal que

$$
A=A \cap \mathbb{R}=I_{1} \cup \cdots \cup I_{m}
$$

tal que $m \leq N$ e $I_{1}, \ldots, I_{m}$ são intervalos reais.

Como $\stackrel{\AA}{A}=\emptyset$, então

$$
I_{i}=\left[a_{i}, a_{i}\right] \text { para cada } i \in\{1, \ldots, m\} .
$$

Logo,

$$
A=\left[a_{1}, a_{1}\right] \cup \cdots \cup\left[a_{m}, a_{m}\right]=\bar{A}
$$

e, assim, $\bar{A}$ não tem ponto interior.

Suponha que o resultado valha para $n$.

Suponha, por absurdo, que exista $A \in C h(\mathcal{S})_{n+1}$ sem ponto interior mas que $\bar{A}$ possui ponto interior. Logo, existem um cubo aberto $B_{0} \subseteq \mathbb{R}^{n}$ e um intervalo aberto real $I$, ambos não vazios, tais que

$$
B_{0} \times I \subseteq \bar{A}
$$

Por (EF5), existe $N \in \mathbb{N}$, tal que, para todo $X \subseteq \mathbb{R}^{n+1}$ afim, $A \cap X$ tem no máximo $N$ componentes conexas. 
Sejam $I_{1}, \ldots, I_{N+1}$ intervalos abertos dois a dois disjuntos tais que $I_{i} \subseteq I$, para $i=$ $1, \ldots, N+1$. Considere

$$
C_{1}=\left\{\bar{x} \in B_{0}: \exists t \in I_{1}(\bar{x}, t) \in A\right\}
$$

Então, $C_{1}$ possui ponto interior.

Com efeito, suponha que não. Como $C_{1} \in C h(\mathcal{S})_{n}$ (pois $C_{1}$ é definido por uma $C h$-fórmula na estrutura $\langle\overline{\mathbb{R}},\{Y: Y \in C h(\mathcal{S})\}\rangle)^{9}$, então, pela hipótese de indução, $\bar{C}_{1}$ não tem ponto interior. Assim, $B_{0} \backslash \bar{C}_{1}$ é aberto e não vazio. Logo, existe um cubo aberto $D_{1}$ não vazio, tal que

$$
D_{1} \subseteq B_{0} \backslash \bar{C}_{1}
$$

Segue, então, que

$$
\emptyset \neq D_{1} \times I_{1} \subseteq B_{0} \times I \subseteq \bar{A} \text { e }\left(D_{1} \times I_{1}\right) \cap A=\emptyset
$$

o que contradiz a definição de fronteira de um conjunto.

Portanto, existe $B_{1}$ cubo aberto tal que $B_{1} \subseteq C_{1}$.

Por indução em $i=1, \ldots, N$, considere

$$
C_{i+1}=\left\{\bar{x} \in B_{i}: \exists t \in I_{i+1}(\bar{x}, t) \in A\right\} .
$$

Assim, obtemos cubos abertos

$$
\emptyset \neq B_{N+1} \subseteq B_{N} \subseteq \cdots \subseteq B_{0}
$$

de modo que,

para todo $\bar{x} \in B_{N+1}$ existem $t_{1} \in I_{1}, \ldots, t_{N+1} \in I_{N+1}$ tais que $\left(\bar{x}, t_{1}\right) \in A, \ldots,\left(\bar{x}, t_{N+1}\right) \in A$.

\footnotetext{
${ }^{9}$ Como $B_{0} \subseteq \mathbb{R}^{n}$ e $I_{1} \subseteq \mathbb{R}$ são semi-algébricos, então $B_{0} \in C h(\mathcal{S})_{n}$ e $I_{1} \in C h(\mathcal{S})_{1}$. Logo, $B_{0}$ e $I_{1}$ são definidos por $C h$-fórmulas $\phi_{B_{0}}(\bar{x})$ e $\phi_{I_{1}}(t)$, respectivamente. De $A \in C h(\mathcal{S})_{n+1}$, segue que $A$ é também definido por uma $C h$-fórmula $\phi_{A}(\bar{x}, y)$. Logo, $C_{1}$ é definido pela $C h$-fórmula $\exists t\left(\phi_{I_{1}}(t) \wedge \phi_{B_{0}}(\bar{x}) \wedge \phi_{A}(\bar{x}, t)\right)$.
} 
Então, $t_{1}, \ldots, t_{N+1} \in A_{\bar{x}}$.

Como $t_{i} \in I_{i}$, para $i=1, \ldots, N+1$, e os intervalos $I_{i}^{\prime} s$ são dois a dois disjuntos, então os $t_{1}, \ldots, t_{N+1}$ são diferentes entre si. Assim, pela definição de $N$,

$A_{\bar{x}}$ contém um intervalo, para $\bar{x} \in B_{N+1}$ arbitrário.

Ou seja,

$$
\text { para todo } \bar{x} \in B_{N+1}, \mu_{1}\left(A_{\bar{x}}\right)>0 \text {. }
$$

Logo,

$$
B_{N+1} \subseteq B=\left\{\bar{x} \in \mathbb{R}^{n}: \mu_{1}\left(A_{\bar{x}}\right)>0\right\}
$$

e, por conseguinte, $\mu_{n}(B)>0$.

Pelo Teorema de Fubini, $\mu_{n+1}(A)>0$. Portanto, pelo Lema 2.1.16, $A$ tem ponto interior. $(\Rightarrow \Leftarrow)$

Como consequência quase imediata dos Lemas 2.1.16 e 2.1.17, logramos a equivalência subsequente.

Corolário 2.1.18. Seja $A \in C h(\mathcal{S})$. Então, as seguintes afirmações são equivalentes:

1. A não tem ponto interior.

2. $\bar{A}$ não tem ponto interior.

3. $\bar{A}$ tem medida nula.

4. A tem medida nula.

Demonstração.

$(1) \Rightarrow(2)$ Lema 2.1.17.

$(2) \Rightarrow(3)$ Lema 2.1 .16 para $\bar{A}$.

$(3) \Rightarrow(4)$ Visto que $A \subseteq \bar{A}$, temos $\mu(A) \leq \mu(\bar{A})=0$. 
$(4) \Rightarrow(1)$ Suponha que $A$ tenha ponto interior. Então, existe um cubo aberto $B$ contido em A. Logo, $0<\mu(B) \leq \mu(A)$. $(\Rightarrow \Leftarrow)$

Como poderá ser observado em resultados posteriores, este último corolário é de préstimo tamanho. Apenas como ilustração, podemos obter a seguinte implicação: se $\bigcup_{i=1}^{n} A_{i}$ tem interior não vazio, então, para algum $j \in\{1, \ldots, n\}, A_{j}$ tem interior não vazio, sendo que $A_{i} \in C h(\mathcal{S})$. De fato, pelo Corolário 2.1.18, segue que $\bigcup_{i=1}^{n} A_{i}$ tem medida não nula. Então, existe $1 \leq j \leq n$ tal que $A_{j}$ tem também medida não nula. Novamente, pelo Corolário 2.1.18, $A_{j}$ tem interior não vazio. Facilmente, vê-se que tal implicação não é, em todo caso, verdadeira. A saber, $\stackrel{\circ}{\mathbb{R}} \neq \emptyset$ e $\stackrel{\mathbb{Q}}{=}=\emptyset=\widehat{\mathbb{R} \backslash \mathbb{Q}}$.

Encerramos esta primeira seção com mais uma propriedade acerca do interior de elementos de $C h(\mathcal{S})$ da qual faremos uso nas seções 2 e 3.

Corolário 2.1.19. Sejam $A, B \in \mathbb{R}^{n}$ tal que $A$ tem ponto interior, $B \in C h(\mathcal{S})$ e não tem ponto interior. Então, $A \cap\left(\mathbb{R}^{n} \backslash B\right)$ tem ponto interior.

Demonstração. Por hipótese,

$$
\stackrel{\circ}{B}=\emptyset \text { e } B \in C h(\mathcal{S})
$$

Logo, pelo Corolário 2.1.18, $\bar{B}$ também não tem ponto interior. Como $\AA \neq \emptyset$, então $\AA \nsubseteq \bar{B}$. Isto significa que existe $\bar{x} \in \AA \cap\left(\mathbb{R}^{n} \backslash \bar{B}\right)$. Uma vez que $\AA \cap\left(\mathbb{R}^{n} \backslash \bar{B}\right)$ é aberto, então $\bar{x}$ é ponto interior deste conjunto. Mas,

$$
\stackrel{\AA}{A}\left(\mathbb{R}^{n} \backslash \bar{B}\right) \subseteq A \cap\left(\mathbb{R}^{n} \backslash B\right)
$$

Assim, $\bar{x}$ é ponto interior de $A \cap\left(\mathbb{R}^{n} \backslash B\right)$.

\subsection{O Teorema da Decomposição Celular (TDC)}

O ponto central desta segunda parte do Capítulo 2, como o próprio título já sugere, consiste no Teorema da Decomposição Celular para a coleção $C h(\mathcal{S})$. Em linhas gerais, esta proposição 
declara que cada subconjunto de $\mathbb{R}^{n}$ em $C h(\mathcal{S})$ pode ser escrito como união finita disjunta de conjuntos "bem comportados", conhecidos como células, e que cada função em $C h(\mathcal{S})$ cujo domínio seja um subconjunto de $\mathbb{R}^{n}$ é "contínua por células". Entretanto, a conclusão do teorema está condicionada à seguinte circunstância: a fronteira dos elementos do fecho Charbonnel de $\mathcal{S}$ está contida em conjuntos de $C h(\mathcal{S})$ de interior nulo.

Encetamos a jornada em direção ao TDC com uma asserção sobre conjuntos em $C h(\mathcal{S})_{n+1}$ que têm interior vazio. A saber, a de que há uma uniformidade no número de pontos de suas respectivas fibras.

Lema 2.2.1 (Limites uniformes das fibras). Seja $C \in C h(\mathcal{S})_{n+1}$ tal que $\stackrel{\circ}{C}=\emptyset$ e seja $B_{0} \subseteq \mathbb{R}^{n}$ um cubo aberto. Então, existem $p \in \mathbb{N} e B \subseteq B_{0}$ cubo aberto tal que, para todo $\bar{x} \in B$, a fibra $C_{\bar{x}}$ contém exatamente $p$ pontos.

Demonstração. Por (EF5), existe $N \in \mathbb{N}$ tal que para qualquer $X \subseteq \mathbb{R}^{n+1}$ afim, $C \cap X$ tem no máximo $N$ componentes conexas.

Para cada $k \in\{1, \ldots, N+1\}$, considere

$$
B_{k}=\left\{\bar{x} \in B_{0}: \exists r_{1} \exists r_{2}>0 \cdots \exists r_{k}>0 \wedge \bigwedge_{i=1}^{k}\left(\bar{x}, r_{1}+\cdots+r_{i}\right) \in C\right\}
$$

Então,

(i) $B_{N+1}$ não possui ponto interior.

Suponhamos que exista $\bar{x} \in B_{N+1}$ ponto interior. Então,

$$
r_{1}, r_{1}+r_{2}, \ldots, r_{1}+\cdots+r_{N+1} \in C_{\bar{x}} \text { são } N+1 \text { pontos distintos. }
$$

Logo, pela definição de $N, C_{\bar{x}}$ contém um intervalo. Assim,

$$
\mu_{1}\left(C_{\bar{x}}\right)>0, \text { para todo } \bar{x} \in B_{N+1}
$$


I. e.,

$$
B_{N+1} \subseteq B^{\prime}=\left\{\bar{x} \in \mathbb{R}^{n}: \mu_{1}\left(C_{\bar{x}}\right)>0\right\}
$$

Logo, pelo Corolário 2.1.18, $\mu_{n}\left(B^{\prime}\right)>0$. Então, por Fubini, $\mu_{n+1}(C)>0$. Assim, novamente pelo Corolário 2.1.18, $C$ tem ponto interior. $(\Rightarrow \Leftarrow)$

(ii) Existe $p \in\{0,1, \ldots, N\}$ tal que $B_{p}$ contém ponto interior, mas $B_{p+1}$ não.

Suponha que, dado $p \in\{0,1, \ldots, N\}$ qualquer, $B_{p}$ tem ponto interior. Então, $B_{p+1}$ também o tem. Como $B_{0}$ é cubo aberto, então tem ponto interior. Logo, $B_{1}$ também o tem. Repetindo este argumento sucessivamente, temos que $B_{N+1}$ tem ponto interior. Contradição com (i).

Como $B_{p+1} \in C h(\mathcal{S})$, então, pelo Corolário 2.1.19,

$$
B_{p} \cap\left(\mathbb{R}^{n} \backslash B_{p+1}\right)=\left(B_{p} \backslash B_{p+1}\right) \text { tem ponto interior. }
$$

Logo, existe $B$ (cubo aberto) tal que $B \subseteq\left(B_{p} \backslash B_{p+1}\right)$. Assim, para todo $\bar{x} \in B$, temos que $\bar{x} \in B_{p}$ e $\bar{x} \notin B_{p+1}$, ou seja, $\left|C_{\bar{x}}\right|=p$.

No que segue, o conjunto das descontinuidades de funções em $C h(\mathcal{S})_{n+1}$ tem interior vazio. Além do mais, é elemento de $C h(\mathcal{S})$.

Lema 2.2.2. Seja $f: A \subseteq \mathbb{R}^{n} \rightarrow \mathbb{R}$ tal que $\operatorname{Gr}(f) \in C h(\mathcal{S})_{n+1}$ e seja

$$
D_{f}=\{\bar{x} \in A: f \text { é descontínua em } \bar{x}\}
$$

\section{Então:}

(a) $D_{f} \in C h(\mathcal{S})_{n}$.

(b) $D_{f}$ não tem ponto interior.

\section{Demonstração.}


(a) Seja $g: A \rightarrow \mathbb{R}$ dada por

$$
\operatorname{Gr}(g)=\{(\bar{x}, y): \exists z((\bar{x}, z) \in G r(f) \wedge z y=1) \vee((\bar{x}, 0) \in G r(f) \wedge y=1)\}^{10} .
$$

Como $\operatorname{Gr}(g)$ é definido por uma $C h$-fórmula em $\langle\overline{\mathbb{R}},\{Y: Y \in C h(\mathcal{S})\}\rangle$, então $\operatorname{Gr}(g) \in$ $C h(\mathcal{S})_{n+1}$.

Considere o seguinte conjunto

$$
E=\{\bar{x} \in A: \exists y \exists r>0((\bar{x}, y) \in \overline{G r(f)} \wedge(\bar{x}, y+r) \in \overline{G r(f)}) \vee(\bar{x}, 0) \in \overline{G r(g)}\}
$$

Como $G r(f) \in C h(\mathcal{S})_{n+1}$ e $A=\pi_{n+1, n}[G r(f)]$, então $A \in C h(\mathcal{S})_{n}$. Logo, $\overline{G r(f)} \in$ $C h(\mathcal{S})_{n+1}$. E, portanto, $E \in C h(\mathcal{S})_{n}$.

Vejamos que $D_{f}=E$.

Seja $\bar{x} \in D_{f}$. Então, $f$ não é contínua em $\bar{x}$. Isto significa que existe $\epsilon>0$, de sorte que para toda vizinhança aberta $U$ de $\bar{x}$, existe $\bar{u} \in U \cap A$ tal que $|f(\bar{u})-f(\bar{x})| \geq \epsilon$. Consequentemente, existe uma sequência $\left(\bar{u}_{i}\right)_{i}$ em $A$, que converge para $\bar{x}$ e tal que,

(*) $\quad$ para todo $i \in \mathbb{N}^{+},\left|f\left(\bar{u}_{i}\right)-f(\bar{x})\right| \geq \epsilon$.

Tomemos a sequência $\left(f\left(\bar{u}_{i}\right)\right)_{i}$ em $\mathbb{R}$. Temos dois casos a considerar:

(i) $\left(f\left(\bar{u}_{i}\right)\right)_{i}$ é ilimitada.

Então,

para todo $j \in \mathbb{N}^{+}$existe $f\left(\bar{u}_{i_{j}}\right) \in\left\{f\left(\bar{u}_{i}\right): i \in \mathbb{N}\right\}$ tal que $\left|f\left(\bar{u}_{i_{j}}\right)\right| \geq j$.

Logo, $f\left(\bar{u}_{i_{j}}\right) \neq 0$, para todo $j$. E, assim $\frac{1}{f\left(\bar{u}_{i_{j}}\right)} \rightarrow 0$. Portanto,

$$
\left(\bar{u}_{i_{j}}, \frac{1}{f\left(\bar{u}_{i_{j}}\right)}\right)_{j} \in G r(g) \text { e }\left(\bar{u}_{i_{j}}, \frac{1}{f\left(\bar{u}_{i_{j}}\right)}\right) \rightarrow(\bar{x}, 0) .
$$

\footnotetext{
${ }^{10} \mathrm{~A}$ função g é pensada como $\frac{1}{f}$.
} 
Então,

$$
(\bar{x}, 0) \in \overline{G r(g)}
$$

ou seja, $\bar{x} \in E$.

(ii) $\left(f\left(\bar{u}_{i}\right)\right)_{i}$ é limitada.

Então, pelo teorema de Bolzano-Weierstrass, $\left(f\left(\bar{u}_{i}\right)\right)_{i}$ possui subsequência convergente, digamos $\left(f\left(\bar{u}_{i_{j}}\right)\right)_{j} \rightarrow a$. Logo, $(\bar{x}, a) \in \overline{G r(f)}$. Além disso, de $(*)$, temos que $f(\bar{x}) \neq a$.

Se $f(\bar{x})>a$, então tomamos $r=f(\bar{x})-a>0$ e, assim,

$$
(\bar{x}, a) \in \overline{G r(f)} \text { e }(\bar{x}, a+r)=(\bar{x}, f(\bar{x})) \in \overline{G r(f)} .
$$

Portanto, $\bar{x} \in E$.

Se $f(\bar{x})<a$, então tomamos $r=a-f(\bar{x})>0$ e, assim,

$$
(\bar{x}, f(\bar{x})) \in \overline{G r(f)} \text { e }(\bar{x}, f(\bar{x})+r)=(\bar{x}, a) \in \overline{G r(f)} .
$$

Portanto, $\bar{x} \in E$.

Agora, seja $\bar{x} \in E$. Então, existem $y$ e $r>0$ tal que

$$
(\bar{x}, y) \in \overline{G r(f)} \text { e }(\bar{x}, y+r) \in \overline{G r(f)} \text { ou }(\bar{x}, 0) \in \overline{G r(g)}
$$

Vejamos que existe uma sequência $\left(\bar{z}_{i}\right)_{i}$ em $A$ tal que $\bar{z}_{i} \rightarrow \bar{x}$, mas $f\left(\bar{z}_{i}\right) \nrightarrow f(\bar{x})$, em ambos os casos.

(i) $\exists y \exists r>0$ tal que $(\bar{x}, y) \in \overline{G r(f)}$ e $(\bar{x}, y+r) \in \overline{G r(f)}$.

Então, existem sequências $\left(\bar{u}_{i}, f\left(\bar{u}_{i}\right)\right)_{i}$ e $\left(\bar{v}_{i}, f\left(\bar{v}_{i}\right)\right)_{i}$ em $G r(f)$ tal que

$$
\left(\bar{u}_{i}, f\left(\bar{u}_{i}\right)\right) \rightarrow(\bar{x}, y) \text { e }\left(\bar{v}_{i}, f\left(\bar{v}_{i}\right)\right) \rightarrow(\bar{x}, y+r)
$$

Se $y=f(\bar{x})$, então tomamos $\left(\bar{v}_{i}\right)_{i} \in A$.

Se $y \neq f(\bar{x})$, basta tomar $\left(\bar{u}_{i}\right)_{i} \in A$.

(ii) $(\bar{x}, 0) \in \overline{G r(g)}$. 
Então, existe $\left(\bar{u}_{i}, g\left(\bar{u}_{i}\right)\right)_{i} \in G r(g)$ tal que $\left(\bar{u}_{i}, g\left(\bar{u}_{i}\right)\right) \rightarrow(\bar{x}, 0)$. Como $g\left(\bar{u}_{i}\right) \rightarrow 0$, então existe um natural $n_{0}$ tal que, para todo $i \geq n_{0},\left|g\left(\bar{u}_{i}\right)-0\right|<1$, ou seja, $f\left(\bar{u}_{i}\right) \neq 0$, para todo $i \geq n_{0}$. Portanto,

para todo $i \geq n_{0}, g\left(\bar{u}_{i}\right)=\frac{1}{f\left(\bar{u}_{i}\right)}$ e existem finitos $i$ 's, com $f\left(\bar{u}_{i}\right)=0$ e $g\left(\bar{u}_{i}\right)=1$.

Considere a subsequência $\left(\bar{u}_{i}, g\left(\bar{u}_{i}\right)\right)_{i \geq n_{0}}$ tal que $g\left(\bar{u}_{i}\right)=\frac{1}{f\left(\bar{u}_{i}\right)}$.

Se $f(\bar{x})=0$, então para $\epsilon=1>0$, existe $n_{\epsilon} \in \mathbb{N}$ tal que

$$
\left|\frac{1}{f\left(\bar{u}_{n}\right)}\right|<1 \text { e, portanto, } 1<\left|f\left(\bar{u}_{n}\right)\right| \text {, para todo } n>n_{\epsilon} .
$$

Se $f(\bar{x}) \neq 0$, então para $0<\epsilon<\frac{1}{|f(\bar{x})|}$, existe $n_{\epsilon} \in \mathbb{N}$ tal que

$$
\left|\frac{1}{f\left(\bar{u}_{n}\right)}\right|<\epsilon \Rightarrow \frac{1}{\epsilon}<\left|f\left(\bar{u}_{n}\right)\right| \Rightarrow \frac{1}{\epsilon}-|f(\bar{x})|<\left|f\left(\bar{u}_{n}\right)\right|-|f(\bar{x})| \leq\left|f\left(\bar{u}_{n}\right)-f(\bar{x})\right|,
$$

para todo $n>n_{\epsilon}$.

Desta forma, em ambos os casos, basta tomarmos a subsequência $\left(\bar{u}_{i}\right)_{i \geq n_{*}} \in A$, sendo que $n_{*}=\max \left\{n_{0}, n_{\epsilon}\right\}$, para que tenhamos: $\bar{u}_{i} \rightarrow \bar{x}$, mas $f\left(\bar{u}_{i}\right) \nrightarrow f(\bar{x})$.

Portanto, $f$ não é contínua em $\bar{x}$. Logo, $\bar{x} \in D_{f}$. Segue, portanto, que $D_{f}=E \in C h(\mathcal{S})_{n}$.

(b) Suponha, por contradição, que $\stackrel{\circ}{D}_{f} \neq \emptyset$. Então, existe um cubo aberto $B$ tal que $B \subseteq D_{f}$

Para cada $m \in \mathbb{N}^{+}$, considere

$$
F_{m}=\{\bar{x} \in B:(-m<f(\bar{x})) \wedge(f(\bar{x})<m)\} .
$$

Então, $\left.F_{m}=B \cap I m(f) \cap\right]-m, m[\in C h(\mathcal{S})$, para todo $m$. Além disso,

$$
\bigcup_{m \in \mathbb{N}^{+}} F_{m}=B .^{11}
$$

\footnotetext{
${ }^{11}$ Dado $\bar{x} \in B$, temos que $f(\bar{x})=0, f(\bar{x})<0$ ou $f(\bar{x})>0$. No primeiro caso, $\left.f(\bar{x}) \in\right]-1,1[$. No segundo caso, $-f(\bar{x})>0$. Assim, existe $m \in \mathbb{N}$ tal que $-f(\bar{x})<m$. Logo, $f(\bar{x})>-m$ e $-m<f(\bar{x})<0<m$. O
} 
Portanto,

$$
\mu_{n}\left(\bigcup_{m \in \mathbb{N}^{+}} F_{m}\right)=\mu_{n}(B)>0
$$

Como consequência, existe $M \in \mathbb{N}^{+}$tal que $\mu_{n}\left(F_{M}\right)>0$. Caso contrário, $\mu_{n}\left(F_{m}\right)=0$, para $m \in \mathbb{N}$ arbitrário. Uma vez que união enumerável de conjuntos de medida nula tem medida nula, segue que $\mu_{n}\left(\bigcup_{m \in \mathbb{N}} F_{m}\right)=0 . \quad(\Rightarrow \Leftarrow)$

Logo, pelo Corolário 2.1.18, $\stackrel{\circ}{F}_{M} \neq \emptyset$. Portanto, existe um cubo aberto $B^{\prime}$ tal que $B^{\prime} \subseteq F_{M}$. Em vista disto, assumimos que $B \subseteq F_{M}$. Por conseguinte,

(†) $\quad$ para todo $\bar{x} \in B,|f(\bar{x})|<M$.

Consequentemente,

qualquer que seja $\bar{x}$ em $B,(\bar{x}, 0) \notin \overline{G r(g)}$.

Com efeito, se existisse, por absurdo, um $\bar{w} \in B$ tal que $(\bar{w}, 0) \in \overline{G r(g)}$, então tomando o aberto $B \times] \frac{-1}{M}, \frac{1}{M}[$ de $(\bar{w}, 0)$, teríamos, pela definição de fecho, que

$$
\text { existem } \bar{x} \in B \text { e } y \in] \frac{-1}{M}, \frac{1}{M}[\text { tal que }(\bar{x}, y) \in G r(g) \text {. }
$$

Assim, $y=\frac{1}{f(\bar{x})}$ ou $y=1$.

Se $y=\frac{1}{f(\bar{x})}$, então, de $\left.y \in\right] \frac{-1}{M}, \frac{1}{M}[$, segue que $f(\bar{x})>M$. Contradição com $(\dagger)$.

Se $y=1$, então, uma vez que $M \geq 1$, temos $y \notin] \frac{-1}{M}, \frac{1}{M}[.(\Rightarrow \Leftarrow)$

Logo,

(†) $\quad$ para todo $\bar{x} \in B$, a fibra $\overline{G r(f)}_{\bar{x}}$ contém pelo menos 2 pontos $^{12}$.

terceiro caso é análogo ao segundo.

${ }^{12}$ Seja $\bar{x} \in B$. Então, de $B \subseteq D_{f}$, temos que $\bar{x} \in D_{f}$. Logo, pelo item (a), $\bar{x} \in E$. Uma vez que $(\bar{x}, 0) \notin \overline{G r(g)}, \forall \bar{x} \in B$, então, existem $y$ e $r>0$ tal que $(\bar{x}, y),(\bar{x}, y+r) \in \overline{G r(f)}$. Ou seja, $\left|\overline{G r(f)_{\bar{x}}}\right| \geq 2$. 
É claro que, $\widehat{\operatorname{Gr}(f)}=\emptyset$, já que $f$ é função. Então, pelo Corolário 2.1.18, $\overline{G r(f)}$ tem interior vazio. Logo, pelo Lema 2.2.1, existem $p \in \mathbb{N}$ e um cubo aberto $B^{\prime} \subseteq B$, de sorte que

$$
\text { para todo } \bar{x} \in B^{\prime},\left|\overline{G r(f)_{\bar{x}}}\right|=p \text {. }
$$

De $(\ddagger), p \geq 2$. Dessa forma, podemos aceitar que $B$ tenha sido reduzido a $B^{\prime}$.

Considere, agora, os seguintes conjuntos

$$
Q_{1}=\{\bar{x} \in B: \exists r>0(\bar{x}, f(\bar{x})+r) \in \overline{G r(f)}\}
$$

e

$$
Q_{2}=\{\bar{x} \in B: \exists r>0(\bar{x}, f(\bar{x})-r) \in \overline{G r(f)}\}
$$

Então, $Q_{1}, Q_{2} \in C h(\mathcal{S})$. Além disso, $Q_{1} \cup Q_{2}=B^{13}$.

Logo, $Q_{1}$ ou $Q_{2}$ tem medida positiva, visto que $\mu_{n}(B)>0$.

Suponha, sem perda de generalidade, que $\mu_{n}\left(Q_{1}\right)>0$. Pelo Corolário 2.1.18, $\stackrel{Q}{1}_{1} \neq \emptyset$. Ou seja, existe um cubo aberto $B^{*}$ contido em $Q_{1}$. Assim, assumimos que $B \subseteq Q_{1}$.

Seja a função $h: B \rightarrow \mathbb{R}$ dada por

$h(\bar{x})=y \Leftrightarrow \bar{x} \in B \wedge(\bar{x}, y) \in \overline{G r(f)} \wedge \exists r_{1}>0 \cdots \exists r_{p-1}>0\left[\bigwedge_{j=1}^{p-1}\left(\bar{x}, y-r_{1}-\cdots-r_{j}\right) \in \overline{G r(f)}\right]$.

\section{Então,}

(i) $h$ é, de fato, uma função, já que $h(\bar{x})=\max \left\{y: y \in \overline{G r(f)_{\bar{x}}}\right\}$.

(ii) $\operatorname{Gr}(h) \in C h(\mathcal{S})$.

(iii) Para todo $\bar{x} \in B, h(\bar{x})>f(\bar{x})$.

\footnotetext{
${ }^{13}$ Seja $\bar{x} \in B$. Então, $\mid{\overline{G r(f)_{\bar{x}}}}_{\mid}=p$, com $p \geq 2$. Uma vez que, $f(\bar{x}) \in{\overline{G r(f)_{\bar{x}}}}_{\text {, então existe } y \in \overline{G r(f)}} \overline{\bar{x}} \mid$ $\{f(\bar{x})\}$. Se $f(\bar{x})>y$, então tomando $r=f(\bar{x})-y>0$, segue que $(\bar{x}, f(\bar{x})-r) \in \overline{G r}(f)$. Se $f(\bar{x})<y$, então tomando $r=y-f(\bar{x})>0$, segue que $(\bar{x}, f(\bar{x})+r) \in \overline{G r(f)}$.
} 
Com efeito, seja $\bar{x} \in B$.

Como $B \subseteq Q_{1}$, então $(\bar{x}, f(\bar{x})),(\bar{x}, f(\bar{x})+r) \in \overline{G r(f)}$, para algum $r>0$. Mas, $\left|\overline{G r(f)_{\bar{x}}}\right|=p$. Logo, tanto $f(\bar{x})$ quanto $f(\bar{x})+r$ precisa ser igual a um dos pontos

$$
h(\bar{x}), h(\bar{x})-r_{1}, \ldots, h(\bar{x})-r_{1}-\cdots-r_{p-1} .
$$

Se $f(\bar{x})=h(\bar{x})$, então $\left|\overline{G r(f)}_{\bar{x}}\right|=p+1$. Contradição.

Portanto,

$$
f(\bar{x})=h(\bar{x})-r_{1}-\cdots-r_{j},
$$

para algum $j \in\{1, \ldots, p-1\}$. Segue, assim, que $h(\bar{x})>f(\bar{x})$.

Considere, agora, para cada $k \in \mathbb{N}^{+}$,

$$
T_{k}=\left\{\bar{x} \in B: h(\bar{x})-f(\bar{x})>\frac{1}{k}\right\}
$$

Como $G r(h), G r(f) \in C h(\mathcal{S})$, então $T_{k} \in C h(\mathcal{S})_{n}$, para todo $k$. Também,

$$
\bigcup_{k \in \mathbb{N}^{+}} T_{k}=B^{14}
$$

Por conseguinte,

$$
\mu_{n}\left(\bigcup_{k \in \mathbb{N}^{+}} T_{k}\right)>0
$$

Logo, existe $K \in \mathbb{N}^{+}$, de sorte que $\mu_{n}\left(T_{K}\right)>0$. Portanto, podemos admitir que $B \subseteq T_{K}$. Consequentemente,

$$
\text { (*) } \forall \bar{x} \in B \exists \bar{u} \in B f(\bar{u})>f(\bar{x})+\frac{1}{K} .
$$

De fato, dado $\bar{x} \in B$, temos, pela definição de $h$, que $(\bar{x}, h(\bar{x})) \in \overline{G r(f)}$. Como $B \subseteq T_{K}$,

\footnotetext{
${ }^{14}$ Dado $\bar{x} \in B$, temos, de (ii), que $h(\bar{x})>f(\bar{x})$. Assim, existe $k \in \mathbb{N}^{+}$tal que $k(h(\bar{x})-f(\bar{x}))>1$. Portanto, $\bar{x} \in T_{k}$.
} 
então

$$
h(\bar{x}) \in] f(\bar{x})+\frac{1}{K}, \infty[
$$

Logo, tomando $B \times] f(\bar{x})+\frac{1}{K}, \infty[$, aberto que contém $(\bar{x}, h(\bar{x}))$, segue $(\star)$.

Agora, fixe $\bar{u}_{0}$.

De $(\dagger), f\left(\bar{u}_{0}\right)<M$. Logo, existe $L \in \mathbb{N}$, tal que

$$
K\left(M-f\left(\bar{u}_{0}\right)\right)<L
$$

i. e.

$$
M<\frac{L}{K}+f\left(\bar{u}_{0}\right)
$$

De $(\star)$, existe $\bar{u}_{1} \in B$ tal que

$$
f\left(\bar{u}_{1}\right)>f\left(\bar{u}_{0}\right)+\frac{1}{K}
$$

Repetindo este processo $L$ vezes, chegamos a uma sequência $\left\{\bar{u}_{0}, \ldots, \bar{u}_{L}\right\} \subseteq B$, de modo que

$$
f\left(\bar{u}_{i+1}\right)>f\left(\bar{u}_{i}\right)+\frac{1}{K}, \text { para } i=1, \ldots, L-1 .
$$

Portanto, $\bar{u}_{L} \in B$ e

$$
f\left(\bar{u}_{L}\right)>f\left(\bar{u}_{L-1}\right)+\frac{1}{K}>f\left(\bar{u}_{L-2}\right)+\frac{2}{K}>\cdots>f\left(\bar{u}_{0}\right)+\frac{L}{K}>M,
$$

o que contradiz $(\dagger)$.

Portanto, $D_{f}$ não tem ponto interior.

Fixaremos alguma notação adequada para o tratamento dos subconjuntos de $\mathbb{R}^{n}$ introduzidos logo em seguida.

\section{Notação.}


1. Para cada $A \in C h(\mathcal{S})_{n}$, escrevemos

$$
C(A)=\left\{f: A \rightarrow \mathbb{R}: f \text { é contínua e } G r(f) \in C h(\mathcal{S})_{n+1}\right\}
$$

$e$

$$
C_{\infty}(A)=C(A) \cup\{-\infty,+\infty\}
$$

em que $\pm \infty$ são pensados como funções constantes em $A$.

2. Para $f, g \in C_{\infty}(A)$, escrevemos

$$
f<g \text { se, e somente se } f(\bar{x})<g(\bar{x}) \text {, para todo } \bar{x} \in A \text {. }
$$

3. Para $f, g \in C_{\infty}(A)$, com $f<g$, escrevemos

$$
(f, g)_{A}=\{(\bar{x}, y) \in A \times \mathbb{R}: f(\bar{x})<y<g(\bar{x})\}
$$

Quando, pelo contexto, ficar claro de que $A$ se trata, escreveremos $(f, g)$ ao invés de $(f, g)_{A}$.

Podemos, então, formalizar o que, antes, chamamos de subconjuntos "bem comportados" de $\mathbb{R}^{n}$.

Definição 2.2.3. Seja $\left(i_{1}, \ldots, i_{n}\right)$ uma sequência de 0's (zeros) e 1's (uns) de comprimento $n$. Uma $\left(i_{1}, \ldots, i_{n}\right)$-célula é um conjunto em $C h(\mathcal{S})_{n}$, obtido por indução em $n$, como segue:

(i) Uma (0)-célula é um conjunto unitário, i. e., $\{r\} \subseteq \mathbb{R}$.

(ii) Uma (1)-célula é um intervalo aberto, i. e., $] a, b[\subseteq \mathbb{R}$, com $a, b \in \mathbb{R} \cup\{ \pm \infty\}$ e $a<b$.

Para $n \geq 1$,

(iii) uma $\left(i_{1}, \ldots, i_{n}, 0\right)$-célula é o gráfico $G r(f)$ de uma função $f \in C(A)$, sendo que $A$ é uma $\left(i_{1}, \ldots, i_{n}\right)$-célula.

(iv) uma $\left(i_{1}, \ldots, i_{n}, 1\right)$-célula é definida como um conjunto da forma $(f, g)_{A}$, em que $A$ é uma $\left(i_{1}, \ldots, i_{n}\right)$-célula, $f, g \in C_{\infty}(A)$ e $f<g$. 
Apenas como ilustração, note que, de acordo com a Definição 2.2.3, uma (0,0)-célula é um ponto $\{(a, b)\}$ do plano $\mathbb{R}^{2}$; uma $(0,1)$-célula é um "intervalo" numa linha vertical $\{a\} \times \mathbb{R}$ e uma (1,0)-célula é o gráfico de uma função contínua definida num intervalo. Daí temos uma noção maior das formas simples que as células assumem.

Dizemos que $A \subseteq \mathbb{R}^{n}$ é uma célula se, e somente se, $A$ é uma $\left(i_{1}, \ldots, i_{n}\right)$-célula, para alguma sequência $\left(i_{1}, \ldots, i_{n}\right) \subseteq\{0,1\}^{n}$, com $n \geq 1$.

Uma célula $A \subseteq \mathbb{R}^{n}$ é aberta se, e somente se, $A$ é aberto em $\mathbb{R}^{n}$.

Consideramos o espaço $\mathbb{R}^{0}=\{0\}$ como uma ()-célula, sendo que () é a sequência de comprimento 0 (nulo). $\mathbb{R}^{0}$ é uma célula aberta.

O lema próximo faz uma descrição de algumas das principais características de uma célula em $\mathbb{R}^{n}$.

\section{Lema 2.2.4.}

1. Se $A \subseteq \mathbb{R}^{n}$ é uma célula, então $\pi_{n, n-1}[A] \subseteq \mathbb{R}^{n-1}$ é uma célula.

2. Todas as células estão em $C h(\mathcal{S})$.

3. Uma célula é aberta se, e somente se, é uma $(1, \ldots, 1)$-célula.

4. Cada célula é homeomorfa a uma célula aberta sob uma função projeção.

5. Cada célula é conexa por caminhos, sendo que estes podem ser escolhidos em $C h(\mathcal{S})$.

Demonstração. A afirmação (3) segue diretamente da definição.

Seja $A \subseteq \mathbb{R}^{n}$ uma $\left(i_{1}, \ldots, i_{n}\right)$-célula. Provaremos, por indução em $n$, as afirmações (1), $(2),(4)$ e $(5)$.

(1) Para $n=1$, temos que ou $A=] a, b[$, com $a, b \in B \cup\{ \pm \infty\}$ e $a<b$, ou $A=\{r\}$, com $r \in \mathbb{R}$. Em qualquer um dos casos, $\pi_{1,0}[A]=\{0\}$ é uma célula.

Suponha $n=k+1$ e que a proposição seja válida para $k$.

Se $i_{n}=0$, então $A=G r(f), \operatorname{com} f: B \rightarrow \mathbb{R}$ e $B$ uma $\left(i_{1}, \ldots, i_{k}\right)$-célula. Logo, 


$$
\pi_{k+1, k}[A]=\pi_{k+1, k}[G r(f)]=B \text { é uma célula. }
$$

Se $i_{n}=1$, então $A=(f, g)_{B}$, com $f, g \in C_{\infty}(B), f<g$ e $B$ uma $\left(i_{1}, \ldots, i_{k}\right)$-célula. Portanto,

$$
\pi_{k+1, k}[A]=\pi_{k+1, k}\left[(f, g)_{B}\right]=B \text { é uma célula. }
$$

(2) Para $n=1$, temos que ou $A=] a, b[$, com $a, b \in B \cup\{ \pm \infty\}$ e $a<b$, ou $A=\{r\}$, com $r \in \mathbb{R}$.

No primeiro caso,

$$
A=(a, b)=\{x \in \mathbb{R}: \overline{\mathbb{R}} \models \phi(x, a, b)\}
$$

$\operatorname{com} \phi(x, y, z)=y<x \wedge x<z$ e parâmetros $a, b \in \mathbb{R}$.

Logo, $A$ é semi-algébrico e, por $(\mathrm{EF} 2), A \in C h(\mathcal{S})_{1}$.

Da mesma forma, temos que $[a, b] \in C h(\mathcal{S})_{1}$ e, portanto, como caso particular, $\{r\} \in$ $C h(\mathcal{S})_{1}$

Suponha, agora, que $n=k+1$ e que as células de comprimento $k$ estejam em $C h(\mathcal{S})_{k}$.

Sabemos que ou $A=G r(f)$, de sorte que $f \in C(B)$ e $B$ é uma $\left(i_{1}, \ldots, i_{k}\right)$-célula, ou $A=(f, g)_{B}$, tal que $f, g \in C_{\infty}(B)$ e $B$ é uma $\left(i_{1}, \ldots, i_{k}\right)$-célula.

No primeiro caso, segue, diretamente da definição de $C(B)$, que $A=G r(f) \in C h(\mathcal{S})_{k+1}$.

Já no segundo caso, temos, pela hipótese de indução e pela definição de $C(B)$, que

$$
\begin{gathered}
(f, g)_{B}=\left\{(\bar{x}, y) \in \mathbb{R}^{k+1}: \bar{x} \in B \text { e } f(\bar{x})<y<g(\bar{x})\right\} \\
=\left\{(\bar{x}, y):\langle\overline{\mathbb{R}},\{Y: Y \in C h(\mathcal{S})\}\rangle \models \exists w \exists z\left(\phi_{B}(\bar{x}) \wedge \phi_{G r(f)}(\bar{x}, w) \wedge \phi_{G r(g)}(\bar{x}, z) \wedge w<y \wedge y<z\right)\right\},
\end{gathered}
$$

em que $\phi_{B}$ é um símbolo predicativo $k$-ário, $\phi_{G r(f)}$ e $\phi_{G r(g)}$ são símbolos predicativos $k+1$-ários e, além disso, $\phi_{B}(\bar{x}), \phi_{G r(f)}(\bar{x}, y)$ e $\phi_{G r(g)}(\bar{x}, y)$ são interpretados em $\langle\overline{\mathbb{R}},\{Y: Y \in C h(\mathcal{S})\}\rangle$, respectivamente, por $\bar{x} \in B,(\bar{x}, y) \in G r(f)$ e $(\bar{x}, y) \in G r(g)$.

Logo, do Corolário 2.1.13, $A=(f, g)_{B} \in C h(\mathcal{S})_{k+1}$. 
(4) Seja $m=i_{1}+\cdots+i_{n}$ e sejam $\lambda(1), \ldots, \lambda(m) \in\{1, \ldots, n\}$ tais que

$$
\lambda(1)<\cdots<\lambda(m) \text { e } i_{\lambda(j)}=1, \text { para } j=1, \ldots, m
$$

Considere $p: \mathbb{R}^{n} \rightarrow \mathbb{R}^{m}$ dada por

$$
p\left(x_{1}, \ldots, x_{n}\right)=\left(x_{\lambda(1)}, \ldots, x_{\lambda(m)}\right) .
$$

Então, por indução em $n$, temos que $A$ é homeomorfo a $p[A] \subseteq \mathbb{R}^{m}$.

(5) Seja $n=1$. Então, $A=\{r\}$ ou $A=] a, b[$, com $r \in \mathbb{R}, a, b \in \mathbb{R} \cup\{ \pm \infty\}$ e $a<b$.

Em ambos os casos, $A$ é um conjunto convexo e, portanto, conexo por caminhos.

Resta mostrar que, dados $x, y \in A$, existe uma função contínua $f:[0,1] \rightarrow A, \operatorname{com} f(0)=x$ e $f(1)=y$, tal que $\operatorname{Gr}(f) \in C h(\mathcal{S})_{2}$.

Com efeito, sejam $x, y \in A$. Seja $f:[0,1] \rightarrow A$ dada por

$$
f(t)=(1-t) x+t y, t \in[0,1] .
$$

Então, $f$ é contínua e, além disso,

$$
G r(f)=\{(t, s): s=f(t)\}=\{(t, s): \overline{\mathbb{R}} \models(s-(1-t) x-t y=0)\}
$$

ou seja, $\operatorname{Gr}(f)$ é um subconjunto semi-algébrico de $\mathbb{R}^{2}$.

Logo, por (EF2), $G r(f) \in C h(\mathcal{S})_{2}$.

Suponha $n=k+1$ e que a proposição valha para $k$. Por definição, temos dois casos a considerar.

(i) $A=G r(f)$ tal que $f: B \rightarrow \mathbb{R}$ é contínua, $G r(f) \in C h(\mathcal{S})$ e $B$ é uma $\left(i_{1}, \ldots, i_{k}\right)$-célula.

De fato, sejam $(\bar{x}, f(\bar{x})),(\bar{z}, f(\bar{z})) \in A$. Então, $\bar{x}, \bar{z} \in B$. Logo, pela hipótese de indução, existe $f_{B}:[0,1] \rightarrow B$ contínua, tal que 


$$
G r\left(f_{B}\right) \in C h(\mathcal{S}) \text { e } f_{B}(0)=\bar{x} \text { e } f_{B}(1)=\bar{z}
$$

Considere a função $f_{A}:[0,1] \rightarrow A$ dada por

$$
f_{A}(t)=\left(f_{B}(t), f \circ f_{B}(t)\right), t \in[0,1]
$$

Então, $f_{A}$ é contínua e

$$
G r\left(f_{A}\right)=\left\{(t, \bar{x}, y) \in \mathbb{R}^{1+k+1}:(t, \bar{x}) \in G r\left(f_{B}\right) \wedge(\bar{x}, y) \in \operatorname{Gr}(f)\right\} \in C h(\mathcal{S})
$$

pois $G r(f), G r\left(f_{B}\right) \in C h(\mathcal{S})$.

Além disso,

$$
f_{A}(0)=\left((\bar{x}, f(\bar{x})) \text { e } f_{A}(1)=(\bar{z}, f(\bar{z})) .\right.
$$

(ii) $A=(f, g)_{B}$ tal que $f, g \in C_{\infty}(B), f<g$ e $B$ é uma $\left(i_{1}, \ldots, i_{k}\right)$-célula.

Aqui, temos alguns subcasos a serem considerados: (a) $f, g \in C(B), f<g$; (b) $f \in C(B)$ e $g=+\infty$; (c) $f=-\infty$ e $g \in C(B)$; e (d) $f=-\infty$ e $g=+\infty$.

(a) Sejam $(\bar{x}, r),(\bar{z}, s) \in(f, g)_{B}$. Então, $\bar{x}, \bar{z} \in B$. Por hipótese de indução, existe $f_{B}:[0,1] \rightarrow B$ contínua tal que

$$
G r\left(f_{B}\right) \in C h(\mathcal{S}) \text { e } f_{B}(0)=\bar{x} \text { e } f_{B}(1)=\bar{z}
$$

Seja $h:[0,1] \rightarrow(f, g)_{B}$ dada por

$$
h(t)= \begin{cases}\left(\bar{x}, r \cdot(1-2 t)+\left(\frac{f(\bar{x})+g(\bar{x})}{2}+r(1-2 t)\right) \cdot(2 t)\right), & 0 \leq t \leq \frac{1}{4} \\ \left.f_{B}(4 t-1), \frac{f\left(f_{B}(4 t-1)\right)+g\left(\left(f_{B}(4 t-1)\right)\right)}{2}\right), & \frac{1}{4} \leq t \leq \frac{1}{2} \\ \left(\bar{z}, \frac{f(\bar{z})+g(\bar{z})}{2} \cdot(1-t)+2 s \cdot\left(t-\frac{1}{2}\right)\right), & \frac{1}{2} \leq t \leq 1\end{cases}
$$

Então, $h$ é contínua, $G r(h) \in C h(\mathcal{S})$ e $h(0)=(\bar{x}, r)$ e $h(1)=(\bar{z}, s)$.

(b) Sejam $(\bar{x}, r),(\bar{z}, s) \in(f, g)_{B}, \operatorname{com} g=+\infty$. Então, $\bar{x}, \bar{z} \in B$. Assim, por hipótese 
de indução, existe $f_{B}:[0,1] \rightarrow B$ contínua, com $f_{B}(0)=\bar{x}$ e $f_{B}(1)=\bar{z}$, e de sorte que $\operatorname{Gr}\left(f_{B}\right) \in C h(\mathcal{S})$.

Se $r=s=\mu$, então tomando $h:[0,1] \rightarrow(f,+\infty)_{B}$ dada por $h(t)=\left(f_{B}(t), \mu\right)$, temos que $h$ é contínua, $\operatorname{Gr}(h) \in C h(\mathcal{S})$ e $h(0)=(\bar{x}, r)$ e $h(1)=(\bar{z}, s)$.

Agora, suponhamos, sem perda de generalidade, que $r<s$.

Considere $h:[0,1] \rightarrow(f,+\infty)_{B}$ dada por

$$
h(t)= \begin{cases}(\bar{x}, r(1-4 t)+(f(\bar{x})+1) \cdot(4 t)), & 0 \leq t \leq \frac{1}{4} \\ \left(f_{B}(4 t-1), f\left(f_{B}(4 t-1)\right)+1\right), & \frac{1}{4} \leq t \leq \frac{1}{2} \\ (\bar{z},(f(\bar{z})+1) \cdot(2-2 t)+s(2 t-1)), & \frac{1}{2} \leq t \leq 1\end{cases}
$$

Então, $h$ é um caminho que liga $(\bar{x}, r)$ a $(\bar{z}, s)$ tal que $G r(h) \in C h(\mathcal{S})$.

Os casos (c) e (d) são análogos.

Definimos, na sequência, um tipo especial de partição de um conjunto.

Definição 2.2.5. Seja $n \geq 1$ e suponha $A \subseteq \mathbb{R}^{n}$.

1. Se $n=1$, uma decomposição celular (ou apenas decomposição) de $A$ é um conjunto finito $\mathcal{E}$ de células em $\mathbb{R}$ duas a duas disjuntas tal que $\bigcup \mathcal{E}=A$.

2. Se $n \geq 2$, uma decomposição celular (ou decomposição) de $A$ é um conjunto finito $\mathcal{E}$ de células em $\mathbb{R}^{n}$ duas a duas disjuntas tal que $\bigcup \mathcal{E}=A$ e o conjunto

$$
\left\{\pi_{n, n-1}[C]: C \in \mathcal{E}\right\}
$$

é uma decomposição de $\pi_{n, n-1}[A]$.

3. Se $B \subseteq A$ e $\mathcal{E}$ é uma decomposição de $A$, então $\mathcal{E}$ particiona (ou é compatível com) $B$ se, para cada célula $C \in \mathcal{E}$,

$$
\text { ou } C \subseteq B \text { ou } C \cap B=\emptyset
$$


Note que, as decomposições de uma célula $C \subseteq \mathbb{R}^{n}$ são as restrições a $C$ das decomposições de $\mathbb{R}^{n}$ que particionam $C$.

Também, se a decomposição $\mathcal{E}$ particiona o conjunto $A$, então podemos escrever $\mathcal{E}$ como $\left\{C_{1}, \ldots, C_{k}, C_{k+1}, \ldots, C_{l}\right\}$, de sorte que

$$
A=C_{1} \cup \cdots \cup C_{k} \text { e } \mathbb{R}^{n} \backslash A=C_{k+1} \cup \cdots \cup C_{l} .
$$

Antes de estabelecermos o TDC, explicitamos a condição de fronteira mencionada no início desta seção.

Suposição 2.2.6. Seja $A \in C h(\mathcal{S})_{n}$, com $n \in \mathbb{N}^{+}$. Então, existe $T \in C h(\mathcal{S})_{n}$ tal que:

(a) $\delta(A) \subseteq T e$,

(b) $\stackrel{\circ}{T}=\emptyset$.

Teorema 2.2.7 (Teorema da Decomposição Celular). Se a Suposição 2.2.6 vale, então valem as seguintes afirmações, para qualquer $n \in \mathbb{N}^{+}$:

$\left(I_{n}\right)$ Dados quaisquer $A_{1}, \ldots, A_{k} \in C h(\mathcal{S})_{n}$ existe uma decomposição de $\mathbb{R}^{n}$ que particiona $\operatorname{cada} A_{1}, \ldots, A_{k}$.

$\left(I I_{n}\right)$ Para cada função $f: A \rightarrow \mathbb{R}\left(A \subseteq \mathbb{R}^{n}\right)$ tal que $G r(f) \in C h(\mathcal{S})_{n+1}$, existe uma decomposição $\mathcal{E}$ de $\mathbb{R}^{n}$ que particiona $A$ e tal que a restrição $f \uparrow C: C \rightarrow \mathbb{R}$, para cada $C \in \mathcal{E}$, com $C \subseteq A$, é contínua.

Demonstração. Vamos provar este resultado por indução em $n$.

$n=1$ :

$\left(I_{1}\right)$ Sejam $A_{1}, \ldots, A_{k} \in C h(\mathcal{S})_{1}$ quaisquer.

Para cada $i=1, \ldots, k$, temos, por (EF5), que os $A_{i}$ 's consistem de um número finito de intervalos e pontos. Portanto, existe um conjunto finito de intervalos abertos e pontos, dois a dois disjuntos, que cobre $\mathbb{R}$ e particiona cada intervalo e ponto que compõe $A_{1}, \ldots, A_{k}$. 
Agora, visto que intervalos e pontos são subconjuntos semi-algébricos de $\mathbb{R}$, então, por (EF2), pertencem a $C h(\mathcal{S})_{1}$.

$\left(I I_{1}\right)$ Seja $f: A \rightarrow \mathbb{R}(A \subseteq \mathbb{R})$ tal que $G r(f) \in C h(\mathcal{S})_{2}$. Chamemos de $D_{f}$ o conjunto das descontinuidades de $f$. Portanto, pelo Lema 2.2.2,

$$
D_{f} \in C h(\mathcal{S})_{1} \text { e } \stackrel{\circ}{D}_{f}=\emptyset .
$$

Logo, por (EF5), $D_{f}$ é um conjunto finito de pontos.

De $\left(I_{1}\right)$, segue que existe uma decomposição $\mathcal{E}$ de $\mathbb{R}$ que particiona $A$ e $D_{f}$. Consequentemente,

$$
\text { para todo } r \in D_{f},\{r\} \in \mathcal{E} \text {. }
$$

Assim, dado $C \in \mathcal{E}$ tal que $C \subseteq A$, temos que

$$
C \neq\{r\} \text {, para todo } r \in D_{f} \text {, ou } C=\{r\} \text {, para algum } r \in D_{f} \text {. }
$$

No primeiro caso, temos $C \subseteq A \backslash D_{f}$ e, portanto, $f \uparrow C$ é contínua.

Já no segundo caso, $f \uparrow C:\{r\} \rightarrow \mathbb{R}$ é, claramente, contínua.

Suponha, agora, que para todo $1 \leq j<n$, as afirmações $\left(I_{j}\right)$ e $\left(I I_{j}\right)$ valham.

Vejamos que $\left(I_{n}\right)$ que vale.

Com efeito, sejam $A_{1}, \ldots, A_{k} \in C h(S)_{n}$ com seus correspondentes $T_{1}, \ldots, T_{k}$, como na Suposição 2.2.6.

AfIRMAÇÃo 1. Seja $\mathcal{E}$ uma decomposição de $\mathbb{R}^{n}$ que particiona $T_{1}, \ldots, T_{k}$ e $A_{1} \cap T_{1}, \ldots, A_{k} \cap T_{k}$. Então, $\mathcal{E}$ também particiona $A_{1}, \ldots, A_{k}$.

Prova da Afirmação. Suponha, por absurdo, que, para algum $i \in\{1, \ldots, k\}, \mathcal{E}$ não particiona $A_{i}$. Isto quer dizer que, existe uma célula $C \in \mathcal{E}$ tal que

$$
C \cap A_{i} \neq \emptyset \text { e } C \nsubseteq A_{i}
$$


Ou seja, existem $\bar{a}, \bar{b} \in C$ tal que

$$
\bar{a} \in A_{i} \text { e } \bar{b} \notin A_{i}
$$

Uma vez que $C$ é conexo por caminhos, então existe $f:[0,1] \rightarrow C$ contínua, tal que $G r(f) \in C h(\mathcal{S})$ e $f(0)=\bar{a}$ e $f(1)=\bar{b}$.

Logo, existe $\bar{x} \in f[[0,1]] \cap \delta\left(A_{i}\right)$.

De fato, $f[[0,1]]$ é conexo. Além disso,

$$
\bar{a} \in f[[0,1]] \cap A_{i} \text { e } \bar{b} \in f[[0,1]] \cap\left(\mathbb{R}^{n} \backslash A_{i}\right) .
$$

Assim, pelo Teorema da Alfândega (ver [14], pág. 99),

$$
f[[0,1]] \cap \delta\left(A_{i}\right) \neq \emptyset .
$$

Como, pela Suposição 2.2.6,

$$
\delta\left(A_{i}\right) \subseteq T_{i}
$$

temos, então, que

$$
\bar{x} \in T_{i}
$$

Assim, $C \cap T_{i} \neq \emptyset$. Consequentemente, $C \subseteq T_{i}$, já que $\mathcal{E}$ particiona $T_{i}$. Portanto, $\bar{a} \in T_{i}$.

Por conseguinte,

$$
C \cap\left(A_{i} \cap T_{i}\right) \neq \emptyset
$$

Novamente, como $\mathcal{E}$ particiona $A_{i} \cap T_{i}$, segue que

$$
C \subseteq A_{i} \cap T_{i}
$$

Logo, $\bar{b} \in A_{i} \cap T_{i}$. Em particular, $\bar{b} \in A_{i}$. $(\Rightarrow \Leftarrow)$ 
Para cada $i=1, \ldots, k$, seja

$$
T_{k+i}=A_{i} \cap T_{i}
$$

Será suficiente encontrar uma decomposição de $\mathbb{R}^{n}$ que particione $T_{1}, \ldots, T_{2 k}$, de acordo com a Afirmação 1.

Considere,

$$
T_{0}=\bigcup_{i=1}^{2 k} T_{i}
$$

Assim, definimos os conjuntos $T_{i}^{j} \subseteq \mathbb{R}^{n-1}$, para cada $i=0,1, \ldots, 2 k$ e $j=1, \ldots, N_{i}+1$, de modo que $N_{i}$ é o número de componentes conexas para $T_{i}$ (como em (EF5)):

$$
T_{i}^{j}=\left\{\bar{x} \in \mathbb{R}^{n-1}: \exists r_{1} \exists r_{2}>0 \cdots \exists r_{j}>0 \bigwedge_{m=1}^{j}\left(\left(\bar{x}, r_{1}+\cdots+r_{m}\right) \in T_{i}\right)\right\}
$$

Note que, $T_{i}^{j} \in C h(\mathcal{S})_{n-1}$ e

$\bar{x} \in T_{i}^{j}$ se, e somente se existem (pelo menos) $j$ pontos na fibra acima de $\bar{x}$ em $T_{i}$.

Agora, aplicamos hipótese de indução $\left(I_{n-1}\right)$ e, assim, obtemos uma decomposição $\mathcal{F}$ de $\mathbb{R}^{n-1}$ que particiona os conjuntos $T_{i}^{j}$ s.

Consequentemente, para cada célula $C \in \mathcal{F}$ e para cada $i \in\{0,1, \ldots, 2 k\}$, existe um único $j_{i}^{C} \in\left\{0,1, \ldots, N_{i},+\infty\right\}$ tal que, para todo $\bar{x} \in C$, a fibra acima de $\bar{x}$ em $T_{i}$ contém exatamente $j_{i}^{C}$ pontos.

De fato, para cada $C \in \mathcal{F}$ e $i \in\{0,1, \ldots, 2 k\}$, definimos

$$
j_{i}^{C}=\left\{\begin{array}{ll}
\max \left\{j \neq N_{i}+1\right\}, & \text { se } C \subseteq T_{i}^{j} \text { e } C \cap T_{i}^{j+1}=\emptyset \\
0, & \text { se } C \cap T_{i}^{1}=\emptyset \\
+\infty, & \text { se } T_{i_{\bar{x}}} \text { contém um intervalo, para } \bar{x} \in C \text { fixado }
\end{array} .\right.
$$

$j_{i}^{C}$ está bem definido, pois:

Pela definição de $\mathcal{F}$, ou $C \subseteq T_{i}^{j}$ ou $C \cap T_{i}^{j}=\emptyset$, para todo $j \in\left\{1, \ldots, N_{i}+1\right\}$. 
Suponha que, para qualquer $j \in\left\{1, \ldots, N_{i}+1\right\}, C \cap T_{i}^{j}=\emptyset$. Então, em particular, $C \cap T_{i}^{1}=\emptyset \mathrm{e}$, assim, $j_{i}^{C}=0$.

Suponha, agora, que exista $j \in\left\{1, \ldots, N_{i}+1\right\}$ tal que $C \subseteq T_{i}^{j}$.

Se $j \neq N_{i}+1$, então tomamos

$$
j^{\prime}=\max \left\{j \in\left\{1, \ldots, N_{i}\right\}: C \subseteq T_{i}^{j}\right\}
$$

e, assim,

$$
C \cap T_{i}^{j^{\prime}+1}=\emptyset
$$

Logo, $j_{i}^{C}=j^{\prime}$.

Se $j=N_{i}+1$, então, para todo $\bar{x} \in C \neq \emptyset, T_{i_{\bar{x}}}$ contém um intervalo.

Com efeito, suponha, por absurdo, que $T_{i_{\bar{x}}}$ não contenha um intervalo. Dessa forma, para cada $m \in\left\{1, \ldots, N_{i}+1\right\}$,

existe $y_{m} \in \mathbb{R}$, de sorte que $r_{1}+\cdots+r_{m}<y_{m}<r_{1}+\cdots+r_{m+1}$ e $y_{m} \notin T_{i_{\bar{x}}}$.

Consequentemente,

$$
\left.T_{i_{\bar{x}}} \subseteq\right]-\infty, y_{1}[\cup] y_{1}, y_{2}[\cup \cdots \cup] y_{N_{i}}, y_{N_{i}+1}[\cup] y_{N_{i}+1},+\infty[
$$

Assim, tomando $X=\{\bar{x}\} \times \mathbb{R} \subseteq \mathbb{R}^{n}$ afim, temos que

$$
T_{i} \cap X=\{\bar{x}\} \times T_{i_{\bar{x}}} \subseteq(\{\bar{x}\} \times]-\infty, y_{1}[) \cup \cdots \cup(\{\bar{x}\} \times] y_{N_{i}+1},+\infty[)
$$

ou seja, $T_{i} \cap X$ tem no mínimo $N_{i}+1$ componentes conexas. $(\Rightarrow \Leftarrow)$

Portanto, para todo $\bar{x} \in C, T_{i_{\bar{x}}}$ contém um intervalo. Logo, $j_{i}^{C}=+\infty$.

Mostraremos, agora, que para cada célula $C \in \mathcal{F}$, podemos encontrar uma decomposição da célula $C \times \mathbb{R} \subseteq \mathbb{R}^{n}$ que particiona $T_{1}, \ldots, T_{2 k}$.

Primeiro, abordamos o caso em que $\stackrel{\circ}{C}=\emptyset$. 
AFIRMAÇÃo 2. Suponha $C \in \mathcal{F}$ e $\stackrel{\circ}{C}=\emptyset$. Então, podemos encontrar uma decomposição da célula $C \times \mathbb{R} \subseteq \mathbb{R}^{n}$ que particiona $T_{1}, \ldots, T_{2 k}$.

Prova da Afirmação. Do Lema 2.2.4, seque que a célula $C$ é homeomorfa a uma célula aberta $p[C] \subseteq \mathbb{R}^{m}$, em que $1 \leq m<{ }^{15} n-1$ e $p$ é uma projeção.

Por conveniência de notação, vamos assumir que a projeção ocorre nas primeiras $m$ coordenadas.

Note que $p[C] \in C h(\mathcal{S})$, já que $p[C]$ é uma célula.

Para cada $i=1, \ldots, 2 k$, seja $T_{i}^{*} \in C h(\mathcal{S})_{m+1}$ dado por

$$
T_{i}^{*}=\left\{\left(x_{1}, \ldots, x_{m}, y\right): \exists z_{m+1} \cdots \exists z_{n-1}\left(x_{1}, \ldots, x_{m}, z_{m+1}, \ldots, z_{n-1}, y\right) \in T_{i}\right\}
$$

Como $m+1<n$, então, por $\left(I_{m+1}\right)$, existe uma decomposição $\mathcal{E}^{*}$ de $\mathbb{R}^{m+1}$ que particiona $T_{1}^{*}, \ldots, T_{2 k}^{*}$ e $p[C] \times \mathbb{R}$.

Para cada célula $G^{*} \in \mathcal{E}^{*}$ tal que $G^{*} \subseteq p[C] \times \mathbb{R}$, formamos o conjunto $G \subseteq \mathbb{R}^{n}$ dado por $G=\left\{\left(x_{1}, \ldots, x_{m}, z_{m+1}, \ldots, z_{n-1}, y\right):\left(x_{1}, \ldots, x_{m}, y\right) \in G^{*} \wedge\left(x_{1}, \ldots, x_{m}, z_{m+1}, \ldots, z_{n-1}\right) \in C\right\}$

Então, $G$ é uma célula.

De fato, cada $G^{*} \in \mathcal{E}^{*}, \operatorname{com} G^{*} \subseteq p[C] \times \mathbb{R} \subseteq \mathbb{R}^{m+1}$, é uma $\left(i_{1}, \ldots, i_{m+1}\right)$-célula.

Se $i_{m+1}=0$, então $G^{*}=G r(f)$, sendo que $f: B \rightarrow \mathbb{R}$ é contínua, $G r(f) \in C h(\mathcal{S})$ e $B$ é uma $\left(i_{1}, \ldots, i_{m}\right)$-célula.

Portanto,

$$
G=G r(f \circ p)
$$

$\operatorname{com} f \circ p: C \rightarrow \mathbb{R}$

Uma vez que $f \circ p$ é contínua (pois, é composição de contínuas) e $G r(f \circ p) \in C h(\mathcal{S})$ (pois,

\footnotetext{
${ }^{15}$ Pois, $\stackrel{\circ}{C}=\emptyset$.
} 
$G$ pode ser descrito por uma $C h$-fórmula), então $G$ é uma célula.

Se $i_{m+1}=1$, então $G^{*}=(f, g)_{B}$, sendo que $f, g \in C_{\infty}(B), f<g$ e $B$ é uma $\left(i_{1}, \ldots, i_{m}\right)$ célula.

Aqui, temos 4 casos a serem considerados: $f, g \in C(B) ; f \in C(B)$ e $g=+\infty ; g \in C(B)$ e $f=-\infty$; e $f=-\infty$ e $g=+\infty$.

(i) $f, g \in C(B), f<g$.

Então, $G=(f \circ p, g \circ p)_{C}, \operatorname{com} f \circ p, g \circ p: C \rightarrow \mathbb{R}$ contínuas e $f \circ p<g \circ p$.

(ii) $f \in C(B), g=+\infty$.

Então, $G=(f \circ p,+\infty)_{C}, \operatorname{com} f \circ p: C \rightarrow \mathbb{R}$ contínua.

(iii) $g \in C(B), f=-\infty$.

Então, $G=(-\infty, g \circ p)_{C}, \operatorname{com} g \circ p: C \rightarrow \mathbb{R}$ contínua.

(iv) $f=-\infty, g=+\infty$.

Então, $G=(-\infty,+\infty)_{C}$.

Em todos estes casos, $G$ é uma célula, já que $G$ é definido (previamente) por uma $C h$ fórmula.

Logo, o conjunto

$$
\left\{G: G^{*} \in \mathcal{E}^{*} \wedge G^{*} \subseteq p[C] \times \mathbb{R}\right\}
$$

é uma decomposição de $C \times \mathbb{R}$ que particiona $T_{1}, \ldots, T_{2 k}$.

Agora, verificamos o caso em que $\stackrel{\circ}{C} \neq \emptyset$.

AFIRMAÇÃo 3. Suponha que $C \in \mathcal{F}$ tenha ponto interior. Então, podemos encontrar uma decomposição da célula $C \times \mathbb{R} \subseteq \mathbb{R}^{n}$ que particiona $T_{1}, \ldots, T_{2 k}$.

Prova da Afirmação. Inicialmente, note que $j_{i}^{C} \neq+\infty$, para todo $i \in\{0,1, \ldots, 2 k\}$. 
Caso contrário, para qualquer $\bar{x} \in C$, a fibra $T_{i \bar{x}}$ conteria um intervalo. E, assim, teríamos

$$
\mu_{1}\left(T_{i \bar{x}}\right)>0
$$

Logo,

$$
C \subseteq B=\left\{\bar{x} \in \mathbb{R}^{n-1}: \mu_{1}\left(T_{i \bar{x}}\right)>0\right\}
$$

Como $\stackrel{\circ}{C} \neq \emptyset$, então $\mu_{n-1}(C)>0$. Consequentemente, $\mu_{n-1}(B)>0$. Pelo Teorema de Fubini, $\mu_{n}\left(T_{i}\right)>0$. Portanto, $T_{i}$ tem ponto interior. $(\Rightarrow \Leftarrow)$

Portanto, para cada $i \in\{0,1, \ldots, 2 k\}$, existe um único $j_{i}^{C} \in\left\{0,1, \ldots, N_{i}\right\}$, o qual chamaremos a partir de agora de $j_{i}$, tal que, para todo $\bar{x} \in C$, a fibra acima de $\bar{x}$ em $T_{i}$ contém exatamente $j_{i}$ pontos.

Considere $J$ o subconjunto de $\{0,1, \ldots, 2 k\}$ tal que $j_{i} \neq 0$, para todo $i \in J$.

Se $J=\emptyset$, então qualquer decomposição de $C \times \mathbb{R}$ particiona $T_{1}, \ldots, T_{2 k}$.

Suponha $J \neq \emptyset$.

Para cada $i \in J$, definimos a função $f_{i}^{l}: C \rightarrow \mathbb{R}, \operatorname{com} l=1, \ldots, j_{i}$, por

$$
f_{i}^{l}(\bar{x})=y \Leftrightarrow \exists z_{1} \cdots \exists z_{j_{i}}\left[z_{1}<\cdots<z_{j_{i}} \wedge \bigwedge_{t=1}^{j_{i}}\left(\left(\bar{x}, z_{t}\right) \in T_{i}\right) \wedge y=z_{l}\right] .
$$

Visto que a fórmula que define $\operatorname{Gr}\left(f_{i}^{l}\right)$ é uma $C h$-fórmula, então $G r\left(f_{i}^{l}\right) \in C h(\mathcal{S})$.

Como $C \in \mathbb{R}^{n-1}$, então aplicando a hipótese de indução $\left(I I_{n-1}\right)$, para cada $f_{i}^{l}$, temos que existe $\mathcal{H}_{i}^{l}$ decomposição de $C$ tal que a restrição de $f_{i}^{l}$ a cada célula de $\mathcal{H}_{i}^{l}$ é contínua. Agora, podemos aplicar a hipótese de indução $\left(I_{n-1}\right)$ para obter uma decomposição $\mathcal{E}(C)$ da célula $C$ que particiona todas as células de todos os $\mathcal{H}_{i}^{l}$ 's.

Seja $G \in \mathcal{E}(C)$. Então, completamos a prova da afirmação construindo uma decomposição de $G \times \mathbb{R}$ que particiona $T_{1}, \ldots, T_{2 k}$.

Se $0 \notin J$, então, para qualquer $\bar{x} \in C, T_{0_{\bar{x}}}=\emptyset$. Logo, $T_{i_{\bar{x}}}=\emptyset$, para todo $i \in\{1, \ldots, 2 k\}$. Em particular, $(G \times \mathbb{R}) \cap T_{i}=\emptyset$, para $i=1, \ldots, 2 k$. Assim, $\{G \times \mathbb{R}\}$ é decomposição de 
$G \times \mathbb{R}$ que particiona $T_{1}, \ldots, T_{2 k}$.

Suponhamos, agora, que $0 \in J$.

Seja

$$
g_{r}=f_{0}^{r} \uparrow G: G \rightarrow \mathbb{R}
$$

para $r=1, \ldots, j_{0}$.

Pela definição de $\mathcal{E}(C)$ e $\mathcal{H}_{0}^{r}$, as $g_{r}$ 's são contínuas. Também, pela definição de $f_{i}^{l}$, segue que

$$
g_{1}<\cdots<g_{j_{0}}
$$

e

$$
\operatorname{Gr}\left(g_{r}\right) \in C h(\mathcal{S})
$$

para $r=1, \ldots, j_{0}$.

Assim,

$$
\mathcal{E}(G)=\left\{\left(-\infty, g_{1}\right)_{G},\left(g_{1}, g_{2}\right)_{G}, \ldots,\left(g_{j_{0}},+\infty\right)_{G}, G r\left(g_{1}\right), \ldots, G r\left(g_{j_{0}}\right)\right\}
$$

é decomposição de $G \times \mathbb{R}$.

Além disso, para cada $i \in\{1, \ldots, 2 k\}$ e cada $l \in\left\{1, \ldots, j_{i}\right\}$, existe $r \in\left\{1, \ldots, j_{0}\right\}$ de sorte que

$$
f_{i}^{l} \uparrow G=g_{r}{ }^{16} .
$$

Portanto, $\mathcal{E}(G)$ particiona $T_{1}, \ldots, T_{2 k}$.

Para cada $C \in \mathcal{F}$, seja $\mathcal{E}(C \times \mathbb{R})$ uma decomposição conforme as Afirmações 2 e 3 .

\footnotetext{
${ }^{16}$ Fixados $i$ e $l \in\left\{1, \ldots, j_{i}\right\}$, temos que, para $\bar{x} \in G,\left(\bar{x}, f_{i}^{l}(\bar{x})\right) \in T_{i} \subseteq T_{0}$. Se $f_{i}^{l}(\bar{x})=f_{0}^{r}(\bar{x})$, para cada $r \in\left\{1, \ldots, j_{0}\right\}$, então $T_{0_{\bar{x}}}$ teria mais de $j_{0}$ pontos, o que é uma contradição. Assim, $f_{i}^{l}(\bar{x}) \neq f_{0}^{r}(\bar{x})$, para algum $r \in\left\{1, \ldots, j_{0}\right\}$.
} 
Então,

$$
\bigcup_{C \in \mathcal{F}} \mathcal{E}(C \times \mathbb{R})
$$

é uma decomposição de $\mathbb{R}^{n}$ que particiona $T_{1}, \ldots, T_{2 k}$.

Logo, pela Afirmação 1, segue $\left(I_{n}\right)$.

Assumimos, agora, que $\left(I_{1}\right), \ldots,\left(I_{n}\right)$ e $\left(I I_{1}\right), \ldots,\left(I I_{n-1}\right)$ valem.

Vejamos que $\left(I I_{n}\right)$ também vale.

Seja $f: A \rightarrow \mathbb{R}\left(A \subseteq \mathbb{R}^{n}\right)$ tal que $\operatorname{Gr}(f) \in C h(\mathcal{S})_{n+1}$.

Considere

$$
D_{f}=\{\bar{x} \in A: f \text { é descontínua em } \bar{x}\}
$$

Pelo Lema 2.2.2, $D_{f} \in C h(\mathcal{S})_{n}$ e $D_{f}=\emptyset$. Por $\left(I_{n}\right)$, existe uma decomposição $\mathcal{E}$ de $\mathbb{R}^{n}$ que particiona $D_{f}$ e $A$.

Basta, então, mostrarmos que se $C$ é uma célula de $\mathcal{E}$ tal que $C \subseteq A$, então podemos obter uma decomposição $\mathcal{H}(C)$ de $C$, de sorte que $f \uparrow B$ é contínua, para cada $B \in \mathcal{H}(C)$.

(i) Suponha $C \in \mathcal{E}, C \subseteq A$ e $\stackrel{\circ}{C} \neq \emptyset$.

Suponha, por absurdo, que $f \uparrow C$ é descontínua. Logo, $C \cap D_{f} \neq \emptyset$. Como $\mathcal{E}$ particiona

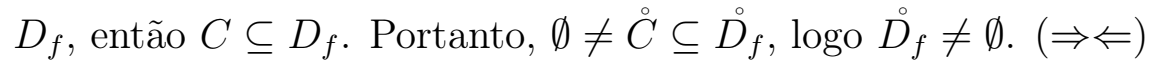

(ii) Suponha $C \in \mathcal{E}, C \subseteq A$ e $\stackrel{\circ}{C}=\emptyset$.

Então, podemos projetar $C$ homeomorficamente em $p[C] \subseteq \mathbb{R}^{m}$, com $m<n$. Novamente, por conveniência de notação, assumimos que a projeção é nas $m$ primeiras coordenadas.

Assim, definimos a função $f^{*}: p[C] \rightarrow \mathbb{R}$ por

$$
\begin{aligned}
\operatorname{Gr}\left(f^{*}\right)=\left\{\left(x_{1}, \ldots, x_{m}, y\right)\right. & :\left(x_{1}, \ldots, x_{m}\right) \in p[C] \wedge \\
& \left.\exists z_{m+1} \cdots \exists z_{n}\left[\left(x_{1}, \ldots, x_{m}, z_{m+1}, \ldots, z_{n}, y\right) \in G r(f)\right]\right\} .
\end{aligned}
$$


Uma vez que $G r\left(f^{*}\right)^{17} \in C h(\mathcal{S})$, segue, da hipótese de indução $\left(I I_{m}\right)$, que existe uma decomposição $\mathcal{H}^{*}$ de $p[C]$, de modo que a restrição de $f^{*}$ a cada célula de $\mathcal{H}^{*}$ é contínua.

Para cada $G^{*} \in \mathcal{H}^{*}$, seja

$$
G=\left(G^{*} \times \mathbb{R}^{n-m}\right) \cap C .
$$

$G$ é uma célula.

De fato, sejam $p^{-1}: p[C] \rightarrow C$ e $\pi: \mathbb{R}^{n} \rightarrow \mathbb{R}^{n-m}$, respectivamente, a inversa da projeção $p$ e a projeção nas $n-m$ últimas coordenadas. Logo,

$$
\pi \circ p^{-1} \uparrow G^{*}: G^{*} \rightarrow \mathbb{R}^{n-m}
$$

é contínua. Além disso,

$$
G=G r\left(\pi \circ p^{-1} \uparrow G^{*}\right)
$$

Agora, note que $G$ pode ser escrito como

$$
\left\{\left(x_{1}, \ldots, x_{m}, z_{m+1}, \ldots, z_{n}\right):\left(x_{1}, \ldots, x_{m}\right) \in G^{*} \wedge\left(x_{1}, \ldots, x_{m}, z_{m+1}, \ldots, z_{n}\right) \in C\right\}
$$

Ou seja, $\operatorname{Gr}\left(\pi \circ p^{-1} \uparrow G^{*}\right) \in C h(\mathcal{S})$. Portanto, $G$ é uma célula.

Assim,

$$
\mathcal{H}(C)=\left\{G: G^{*} \in \mathcal{H}^{*}\right\}
$$

é uma decomposição de $C$.

Por último, temos que $f=f^{*} \circ p$, em $C$.

Como $G \subseteq C$, então $p$ é contínua em $G$. Também, $p[G] \subseteq G^{*}$. Logo, pela definição de $\mathcal{H}^{*}$, $f^{*}$ é contínua em $p[G]$. Portanto, $f^{*} \circ p$ é contínua em $G$. Assim, para todo $G \in \mathcal{H}(C), f$ é contínua em $G$.

Portanto, $\left(I I_{n}\right)$ vale.

\footnotetext{
${ }^{17}$ Como $z_{m+1}, \ldots, z_{n}$ são únicos, logo o conjunto ao qual $G r\left(f^{*}\right)$ se refere é gráfico de alguma função.
} 
Ressaltamos que a consequência do TDC (para $C h(\mathcal{S}))$ na qual estaremos interessados é a propriedade de $C h(\mathcal{S})$ ser fechado sob o complemento. Precisamente, usaremos este fato no Teorema 2.5.7.

\subsection{Seleção de uma função de classe $\mathcal{C}^{p}$}

Nesta seção desenvolveremos mais algumas particularidades de $C h(\mathcal{S})$, que serão utilizadas posteriormente.

Começamos com um resultado que diz respeito a determinados conjuntos de $C h(\mathcal{S})$, conhecido como seleção de uma função contínua. Mais especificamente, mostraremos que dado $A \in C h(\mathcal{S})_{n+m}$, podemos encontrar uma função contínua, em $C h(\mathcal{S})$, cujo domínio é um cubo aberto do $\mathbb{R}^{n}$ e cujo gráfico está contido em $A$, contanto que o interior de $\pi_{n+m, n}[A]$ seja não vazio. Ao final, apresentamos, sob as mesmas hipóteses, uma versão mais forte desta proposição, que é a seleção de uma função $\mathcal{C}^{p}$, sendo que $p \in \mathbb{N}$. Para tanto, estabeleceremos lemas, no decurso da seção, que serão suficientes para garantir nosso intento.

Lema 2.3.1 (Seleção de uma função contínua). Sejam $n, m \in \mathbb{N}^{+}, A \in C h(\mathcal{S})_{n+m}$ e um cubo $B \subseteq \mathbb{R}^{n}$ tal que

$$
\forall \bar{x} \in B \exists \vec{y} \in \mathbb{R}^{m}(\bar{x}, \vec{y}) \in A .
$$

Então, existem um cubo $B^{\prime} \subseteq B$ e uma função continua $f: B^{\prime} \rightarrow \mathbb{R}^{m}$ contida em $C h(\mathcal{S})_{n+m}$ (i. e., $\left.\operatorname{Gr}(f) \in C h(\mathcal{S})_{n+m}\right)$ de modo que $\operatorname{Gr}(f) \subseteq A$.

Demonstração. Sejam $A \in C h(\mathcal{S})_{n+m}$ e um cubo $B \subseteq \mathbb{R}^{n}$ com a propriedade: para todo $\bar{x} \in B$ existe $\vec{y} \in \mathbb{R}^{m}$, de modo que $(\bar{x}, \vec{y}) \in A$.

AFIRMAÇÃo. Sob as hipóteses apresentadas, existem um cubo $\tilde{B} \subseteq B$ e uma função (não necessariamente contínua) $f: \tilde{B} \rightarrow \mathbb{R}^{m}$ tal que $\operatorname{Gr}(f) \in C h(\mathcal{S})_{n+m}$ e $\operatorname{Gr}(f) \subseteq A$.

Prova da Afirmação. Vamos proceder por indução em $m$.

Seja $m=1$.

Se $A \cap(B \times \mathbb{R})$ tem um ponto interior, então existem um cubo $\tilde{B} \subseteq B$ e um ponto $b \in \mathbb{R}$ tal que $\tilde{B} \times\{b\} \subseteq A$. 
Seja $f: \tilde{B} \rightarrow \mathbb{R}$ a função dada por

$$
f(\bar{x})=b, \text { para todo } \bar{x} \in \tilde{B} \text {. }
$$

Assim, $G r(f)=\tilde{B} \times\{b\} \in C h(\mathcal{S})$.

Se $A \cap(B \times \mathbb{R})$ não tem ponto interior, então, pelo Lema 2.2 .1 , existem um cubo $\tilde{B} \subseteq B$ e um natural $k$, de sorte que, para todo $\bar{x} \in \tilde{B}$

$$
\left|(A \cap(B \times \mathbb{R}))_{\bar{x}}\right|=\left|A_{\bar{x}}\right|=k>0,
$$

pela hipótese sobre $A$.

Seja $f: \tilde{B} \rightarrow \mathbb{R}$ a função dada por

$$
G r(f)=\left\{\left(\bar{x}, r_{1}\right) \in \mathbb{R}^{n+1}: \bar{x} \in \tilde{B} \wedge \exists r_{2}>0 \cdots \exists r_{k}>0 \bigwedge_{i=1}^{k}\left(\left(\bar{x}, r_{1}+\cdots+r_{i}\right) \in A\right)\right\} .
$$

Então, $G r(f) \in C h(\mathcal{S})$ e, pela própria definição de $G r(f), G r(f) \subseteq A$.

Suponha que $m>1$ e que a afirmação seja válida para os naturais menores que $m$.

Então, para $m-1\left(\right.$ e $\pi_{n+m, n+m-1}[A]$ e $\left.B\right)$, existem um cubo $B^{*} \subseteq B$ e uma função $f^{*}: B^{*} \rightarrow \mathbb{R}^{m-1}$ tais que

$$
G r\left(f^{*}\right) \subseteq C h(\mathcal{S}) \text { e } G r\left(f^{*}\right) \subseteq \pi_{n+m, n+m-1}[A]
$$

Considere a relação $S$ dada por

$$
S=\left\{(\bar{x}, y) \in \mathbb{R}^{n+1}: \bar{x} \in B^{*} \wedge\left(\bar{x}, f^{*}(\bar{x}), y\right) \in A\right\}
$$

Logo, pela hipótese de indução para $m=1$ (e $S$ e $\left.B^{*}\right)$, existem um cubo $\tilde{B} \subseteq B^{*}$ e uma função $\tilde{f}: \tilde{B} \rightarrow \mathbb{R}$ tal que

$$
G r(\tilde{f}) \subseteq S \text { e } G r(\tilde{f}) \in C h(\mathcal{S})_{n+1}
$$


Definimos, então, a função $f: \tilde{B} \rightarrow \mathbb{R}^{m}$ por

$$
G r(f)=\left\{\left(\bar{x}, y_{1}, \ldots, y_{m}\right) \in \mathbb{R}^{n+m}: \bar{x} \in \tilde{B} \wedge f^{*}(\bar{x})=\left(y_{1}, \ldots, y_{m-1}\right) \wedge \tilde{f}(\bar{x})=y_{m}\right\}
$$

Segue, diretamente da definição de $G r(f)$, que $G r(f) \subseteq A$. Também, $G r(f) \in C h(\mathcal{S})_{n+m}$.

Então, pela Afirmação, existem um cubo $\tilde{B} \subseteq B$ e uma função $f: \tilde{B} \rightarrow \mathbb{R}^{m}$ tal que

$$
G r(f) \in C h(\mathcal{S})_{n+m} \text { e } G r(f) \subseteq A .
$$

Para cada $j=1, \ldots, m$, definimos a função $f_{j}: \tilde{B} \rightarrow \mathbb{R}$ por

$$
f_{j}(\bar{x})=y_{j} \Leftrightarrow f(\bar{x})=\left(y_{1}, \ldots, y_{m}\right)
$$

Seja

$$
D_{f_{j}}=\left\{\bar{x} \in \tilde{B}: f_{j} \text { é descontínua em } \bar{x}\right\} .
$$

Chamemos de $D$ a união $D_{f_{1}} \cup \cdots \cup D_{f_{m}}$.

Pelo Lema 2.2.2, $D_{f_{j}} \in C h(\mathcal{S})$, para todo $j$. Logo, $D \in C h(\mathcal{S})$. Ainda pelo Lema 2.2.2, $D_{f_{j}}=\emptyset$. Assim, $D_{f_{j}}$ tem medida nula, para todo $j$. Então, $D$ tem medida nula, o que implica $\stackrel{\circ}{D}=\emptyset$. Logo, pelo Corolário 2.1.19, $\tilde{B} \cap\left(\mathbb{R}^{n} \backslash D\right)=\tilde{B} \backslash D$ tem um ponto interior. Por conseguinte, existe um cubo $B^{\prime} \subseteq(\tilde{B} \backslash D)$. Consequentemente, $f_{j} \uparrow B^{\prime}$ é contínua, para todo $j$. Portanto, $f \uparrow B^{\prime}=\left(f_{1} \uparrow B^{\prime}, \ldots, f_{m} \uparrow B^{\prime}\right)$ é contínua.

A seguir, inserimos um resultado que será usado tanto no lema ulterior quanto na proposição principal da Seção 4.

Lema 2.3.2. Seja $A \in C h(\mathcal{S})_{n+1}$ tal que

$$
\left.A \subseteq \mathbb{R}^{n} \times\right] 0, \infty[\text { e } \stackrel{\AA}{A}=\emptyset
$$

Seja $A_{0}=\{\bar{x}:(\bar{x}, 0) \in \bar{A}\}$. Então, $\AA_{0}=\emptyset$. 
Demonstração. Em primeiro lugar, note que $A_{0} \in C h(\mathcal{S})$.

Suponha, por absurdo, que $\AA_{0} \neq \emptyset$. Então, existe um cubo aberto tal que $B_{0} \subseteq A_{0}$. Como $\AA=\emptyset$, logo, pelo Lema 2.2.1, existem $k \in \mathbb{N}$ e um cubo $B_{1} \subseteq B_{0}$ de modo que

$$
\text { para todo } \bar{x} \in B_{1},\left|A_{\bar{x}}\right|=k \text {. }
$$

Analogamente ao caso $m=1$, na demonstração da Afirmação do Lema 2.3.1, existe uma função $f: B_{1} \rightarrow \mathbb{R}$ tal que $G r(f) \in C h(\mathcal{S}), G r(f) \subseteq A$ e

$$
\text { (†) } \quad \text { para todo } \bar{x} \in B_{1},(\bar{x}, y) \in A \text { implica } f(\bar{x}) \leq y
$$

Também, analogamente à parte final da demonstração do Lema 2.3.1, existe um cubo $B_{2} \subseteq B_{1}$ tal que $f \uparrow B_{2}$ é contínua.

Podemos, então, tomar $B_{2}$ como um cubo fechado. Como $B_{2}$ é um compacto, segue que $f \uparrow B_{2}$ possui valor mínimo $\epsilon$. Uma vez que $\left.\operatorname{Gr}(f) \subseteq A \subseteq \mathbb{R}^{n} \times\right] 0, \infty[$, temos $\epsilon>0$.

Vejamos, então, que existe $\bar{x} \in B_{2}$, tal que $(\bar{x}, 0) \notin \bar{A}$.

De fato, como $\stackrel{\circ}{B}_{2} \neq 0$, então existe $\bar{x} \in \stackrel{\circ}{B}_{2}$. Agora, suponha, por absurdo, que $(\bar{x}, 0) \in \bar{A}$. Então, existe uma sequência $\left(\bar{z}_{j}, s_{j}\right)_{j}$ em $A$ tal que $\bar{z}_{j} \rightarrow \bar{x}$ e $s_{j} \rightarrow 0$.

De $\bar{z}_{j} \rightarrow \bar{x}$, temos que existe $J_{0} \in \mathbb{N}$, de sorte que $\bar{z}_{j} \in \stackrel{\circ}{B}_{2}$, para todo $j>J_{0}$.

De $s_{j} \rightarrow 0$, segue que existe $J_{1} \in \mathbb{N}$, de modo que $s_{j}<\epsilon$, para todo $j>J_{1}$.

Tome $J=\max \left\{J_{0}, J_{1}\right\}$. Assim, para todo $j>J$,

$$
\bar{z}_{j} \in \stackrel{\circ}{B}_{2} \mathrm{e}\left(\bar{z}_{j}, s_{j}\right) \in A
$$

Logo, de $(\dagger), f\left(\bar{z}_{j}\right) \leq s_{j}$. Assim, $f\left(\bar{z}_{j}\right)<\epsilon$. Contradição, pois $\epsilon \leq f(\bar{z})$, para qualquer $\bar{z} \in B_{2}$.

Portanto, $(\bar{x}, 0) \notin \bar{A}$.

Como consequência imediata da definição de $A_{0}, \bar{x} \notin A_{0}$. Ou seja, $B_{2} \not A_{0} .(\Rightarrow \Leftarrow)$ 
O restante desta seção consiste de lemas para o Teorema 2.3.14, que é o da seleção de uma função $\mathcal{C}^{p}$. Este, por sua vez, será usado na demonstração da Afirmação 6, no Teorema 2.4.2. Notação. Se $A \subseteq \mathbb{R}^{n+1}$, então $(\bar{a},+\infty) \in \bar{A}$ denota

$$
(\bar{a}, 0) \in \overline{\{(\bar{x}, z): \exists y((\bar{x}, y) \in A \wedge y z=1 \wedge z>0)\}}
$$

$e(\bar{a},-\infty) \in \bar{A}$ denota

$$
(\bar{a}, 0) \in \overline{\{(\bar{x}, z): \exists y((\bar{x}, y) \in A \wedge y z=1 \wedge z<0)\}}
$$

Lema 2.3.3. Seja $h: B \times(0, \lambda) \rightarrow \mathbb{R}$ uma função, com $B \subseteq \mathbb{R}^{n}$ cubo aberto e $\lambda>0$, tal que $G r(h) \in C h(\mathcal{S})_{n+2}$ e $h$ é contínua. Seja

$$
S=\left\{\bar{x}: \exists y_{1} \exists y_{2} \in \mathbb{R}_{ \pm \infty}\left(y_{1} \neq y_{2} \wedge\left(\bar{x}, 0, y_{1}\right) \in \overline{G r(h)} \wedge\left(\bar{x}, 0, y_{2}\right) \in \overline{G r(h)}\right)\right\}
$$

$\operatorname{com} \mathbb{R}_{ \pm \infty}=\mathbb{R} \cup\{ \pm \infty\}$. Então, $S \in C h(\mathcal{S})$ e $\stackrel{\circ}{S}=\emptyset$

Demonstração. A expressão

$$
\exists y_{1} \exists y_{2}\left(y_{1}<y_{2} \vee y_{2}<y_{1} \wedge \bar{\phi}_{G r(h)}\left(\bar{x}, 0, y_{1}\right) \wedge \bar{\phi}_{G r(h)}\left(\bar{x}, 0, y_{2}\right)\right)
$$

com $\phi_{G r(h)}\left(\bar{x}, 0, y_{i}\right)$ interpretado como $\left(\bar{x}, 0, y_{i}\right) \in G r(h)$, é uma $C h$-fórmula que define $S$. Então, $S \in C h(\mathcal{S})$.

Vejamos que $\stackrel{\circ}{S}=\emptyset$.

AfirmaÇÃo. Suponha $(\bar{a}, 0, b),(\bar{a}, 0, c) \in \overline{G r(h)}$, com $b, c \in \mathbb{R}_{ \pm \infty}$ e $b<c$. Então,

$$
(\bar{a}, 0, z) \in \overline{G r(h)}, \text { para todo } z \in[b, c] .
$$

Prova da Afirmação. É suficiente mostrar que dados $d \in] b, c[, V \subseteq B$ um cubo aberto contendo $\bar{a}$ e $\eta \in] 0, \lambda[$ arbitrários, existe $(\bar{x}, \gamma) \in V \times] 0, \eta[$ tal que $(\bar{x}, \gamma, d) \in G r(h)$.

Fixemos $d \in] b, c[, V \subseteq B$ um cubo aberto contendo $\bar{a}$ e $\eta \in] 0, \lambda[$. 
Como $(\bar{a}, 0, b) \in \overline{G r(h)}$ e $b<d$, então para $V \times]-\eta, \eta] \times]-\infty, d[$, aberto que contém $(\bar{a}, 0, b)$, existe um ponto $\left(\bar{x}^{\prime}, \gamma^{\prime}, z^{\prime}\right)$ em $(V \times]-\eta, \eta[\times]-\infty, d[) \cap G r(h)$. Isto implica que,

$$
\left.\left(\bar{x}^{\prime}, \gamma^{\prime}, z^{\prime}\right) \in V \times\right] 0, \eta[\times]-\infty, d\left[\text { e } h\left(\bar{x}^{\prime}, \gamma^{\prime}\right)=z^{\prime}\right.
$$

Similarmente, como $(\bar{a}, 0, c) \in \overline{G r(h)}$ e $d<c$, então existe $\left(\bar{x}^{\prime \prime}, \gamma^{\prime \prime}, z^{\prime \prime}\right)$ tal que

$$
\left.\left(\bar{x}^{\prime \prime}, \gamma^{\prime \prime}, z^{\prime \prime}\right) \in V \times\right] 0, \eta[\times] d, \infty\left[\text { e } h\left(\bar{x}^{\prime \prime}, \gamma^{\prime \prime}\right)=z^{\prime \prime}\right.
$$

Como $V \times(0, \eta)$ é convexo, então contém o segmento $L$ que liga $\left(\bar{x}^{\prime}, \gamma^{\prime}\right)$ a $\left(\bar{x}^{\prime \prime}, \gamma^{\prime \prime}\right)$. Logo,

$$
L \subseteq B \times] 0, \lambda[
$$

Como $h\left(\bar{x}^{\prime}, \gamma^{\prime}\right)=z^{\prime}<d<z^{\prime \prime}=h\left(\bar{x}^{\prime \prime}, \gamma^{\prime \prime}\right)$, então, pelo Teorema do Valor Intermediário,

$$
\text { existe }(\bar{x}, \gamma) \in L \subseteq V \times] 0, \eta[\text { tal que } h(\bar{x}, \gamma)=d
$$

i. e.,

$$
(\bar{x}, \gamma, d) \in G r(h)
$$

Segue da Afirmação, que para todo $\bar{x} \in S$ existem $y_{1} \neq y_{2}$ (que supomos, sem perda de generalidade, $\left.y_{1}<y_{2}\right)$ tais que

$$
\left[y_{1}, y_{2}\right] \subseteq \overline{\operatorname{Gr}(h)}_{(\bar{x}, 0)}
$$

Então, $\mu_{1}\left(\overline{\operatorname{Gr}(h)}_{(\bar{x}, 0)}\right)>0$.

Agora, suponha, por absurdo, que $S$ tenha interior não vazio. Então, $S$ contém um cubo $B^{\prime}$. Consequentemente,

$$
B^{\prime} \subseteq\left\{\bar{x}: \mu_{1}\left(\overline{G r(h)}_{(\bar{x}, 0)}\right)>0\right\}=S .
$$

Portanto, $\mu_{n}(S)>0$. 
Por Fubini, $A_{0}=\{(\bar{x}, y):(\bar{x}, 0, y) \in \overline{G r(h)}\}$ tem medida positiva. Logo, $A_{0}$ tem interior não vazio. Então, pelo Lema 2.3.2, $G r(h)$ também tem interior não vazio. Absurdo, pois $h$ é função.

Introduziremos, agora, subconjuntos do $\mathbb{R}^{n+m}$ que são "quase funções". Mais precisamente, exigimos que cada elemento $\bar{x}$ de um determinado subconjunto $U \subseteq \mathbb{R}^{n}$ esteja associado a um elemento $\vec{y}$ de $\mathbb{R}^{m}$. É permitido que esta associação não seja, necessariamente, unívoca. Todavia, o conjunto dos pontos de $U$ que têm mais de um correspondente possui medida nula.

Definição 2.3.4. Seja $A \subseteq U \times \mathbb{R}^{m}$, com $U \subseteq \mathbb{R}^{n}$, tal que:

1. para todo $\bar{x} \in U$ existe $\vec{y} \in \mathbb{R}^{m},(\bar{x}, \vec{y}) \in A$.

2. $\left\{\bar{x} \in U: \exists y_{1} \exists y_{2}\left(y_{1} \neq y_{2} \wedge\left(\bar{x}, y_{1}\right) \in A \wedge\left(\bar{x}, y_{2}\right) \in A\right)\right\}$ é $\mu_{n}$-nulo.

Então, dizemos que $A$ é o gráfico de uma pseudofunção.

\section{Notação.}

1. Pseudofunções serão denotadas por $\hat{f}, \hat{g}$, etc. e, analogamente às funções, usaremos as notações $\hat{f}: U \rightarrow \mathbb{R}^{m}$ e $G r(\hat{f})$ como sinônimos do conjunto $A$ da Definição 2.3.4.

2. Se $\bar{a} \in U$ e existe um único $\vec{y} \in \mathbb{R}^{m}$ tal que $(\bar{a}, \vec{y}) \in G r(\hat{f})$, então escrevemos $\hat{f}(\bar{a})=\vec{y}$.

3. Sejam $\varepsilon \in \mathbb{R}, n \in \mathbb{N}^{+}$e $i \in\{1, \ldots, n\}$. Então, $\bar{\varepsilon}_{i}$ denota a $n$-upla $(0, \ldots, 0, \varepsilon, 0, \ldots, 0)$, de modo que é é a i-ésima coordenada.

Lema 2.3.5. Seja $U \subseteq \mathbb{R}^{n}$.

(i) Qualquer função $f: U \rightarrow \mathbb{R}^{m}$ é uma pseudofunção.

(ii) Seja $\hat{f}: U \rightarrow \mathbb{R}^{m}$ uma pseudofunção tal que $U$ é aberto e $G r(\hat{f}) \in C h(\mathcal{S})$, então existe um cubo aberto $B \subseteq \mathbb{R}^{n}$ tal que $\hat{f} \uparrow B$ é uma função. 


\section{Demonstração.}

(i) É imediato das definições de função e pseudofunção.

(ii) Seja $B^{\prime}=\left\{\bar{x} \in U: \exists y_{1} \exists y_{2}\left(y_{1} \neq y_{2} \wedge\left(\bar{x}, y_{1}\right) \in \operatorname{Gr}(\hat{f}) \wedge\left(\bar{x}, y_{2}\right) \in \operatorname{Gr}(\hat{f})\right)\right\}$.

Dado que $\hat{f}$ é pseudofunção, então $\stackrel{\circ}{B}^{\prime}=\emptyset$. Além disso, como $\operatorname{Gr}(\hat{f}) \in C h(\mathcal{S}), \operatorname{logo}$ $B^{\prime} \in C h(\mathcal{S})$. Assim, pelo Corolário 2.1.19, $U \cap\left(\mathbb{R}^{n} \backslash B^{\prime}\right)$ tem interior não vazio, ou seja, existe $B \subseteq\left(U \backslash B^{\prime}\right)$ cubo aberto. Portanto, $\hat{f} \uparrow B$ é uma função.

Aviso. Daqui até o fim do Lema 2.3.11, fixamos uma pseudofunção $\hat{f}: U \rightarrow \mathbb{R}$, com $U \subseteq \mathbb{R}^{n}$ aberto, tal que $\operatorname{Gr}(\hat{f}) \in C h(\mathcal{S})$.

Definição 2.3.6. Seja $\hat{f}: U \rightarrow \mathbb{R}$ uma pseudofunção como a fixada logo acima. Sejam $\bar{a} \in U$ e um vetor $\bar{v} \in \mathbb{R}^{n}$. Então, dizemos que a derivada direcional de $\hat{f}$, em $\bar{a}$, na direção $\bar{v}$ existe se existe um aberto $V \subseteq U$, com $\bar{a} \in V$, tal que $\hat{f}$ é função em $V$ e o limite

$$
\frac{\partial \hat{f}}{\partial \bar{v}}(\bar{a})=\lim _{\varepsilon \rightarrow 0} \frac{\hat{f}(\bar{a}+\varepsilon \bar{v})-\hat{f}(\bar{a})}{\varepsilon}
$$

existe.

Em seguida, definimos pseudofunções, a partir da pseudofunção fixada $\hat{f}: U \rightarrow \mathbb{R}$, que serão úteis na tentativa de investigar os pontos nos quais $\hat{f}$ é uma função $\mathcal{C}^{1}$.

Definição 2.3.7. Para cada $i=1, \ldots, n$, seja $\hat{g}_{i}$ a pseudofunção dada por

$$
\begin{aligned}
\operatorname{Gr}\left(\hat{g}_{i}\right)=\{(\bar{x}, \varepsilon, y): \bar{x} \in U & \wedge \bar{x}+\bar{\varepsilon}_{i} \in U \wedge \exists z_{1} \exists z_{2}\left[\left(\bar{x}, z_{1}\right) \in G r(\hat{f})\right. \\
& \left.\left.\wedge\left(\bar{x}+\bar{\varepsilon}_{i}, z_{2}\right) \in G r(\hat{f}) \wedge \varepsilon y=z_{2}-z_{1}\right] \wedge(\varepsilon \neq 0)\right\} .
\end{aligned}
$$

Aqui, notamos que é um tanto difícil escrevermos de forma explícita o domínio das pseudofunções $\hat{g}_{i}$, uma vez que não conseguimos determinar um intervalo $I \subseteq \mathbb{R}$, de modo que para quaisquer $\varepsilon \in I \backslash\{0\}$ e $\bar{x} \in U$, tenhamos $\bar{x}+\bar{\varepsilon} \in U$. Entretanto, podemos delimitar "domínios locais" para $\hat{g}_{i}$, como assegura o lema seguinte.

Lema 2.3.8. Seja $\hat{g}_{i}$ uma pseudofunção como na Definição 2.3.7. 
1. Se $\bar{x} \in U$, então existem $V \subseteq U$ um cubo, com $\bar{x} \in V$, e um $\eta>0$ tal que $\hat{g}_{i}$ está definida, como pseudofunção, em $V \times(]-\eta, \eta[\backslash\{0\})$.

2. Seja $G_{i}=\left\{(\bar{x}, y):(\bar{x}, 0, y) \in \overline{G r\left(\hat{g}_{i}\right)}\right\}$. Então, $\operatorname{Gr}\left(\hat{g}_{i}\right), G_{i} \in C h(\mathcal{S})$.

3. $G_{i}$ é fechado.

4. Se $\bar{a} \in U$ e $\frac{\partial \hat{f}}{\partial x_{i}}(\bar{a})$ existe, então $\left(\bar{a}, \frac{\partial \hat{f}}{\partial x_{i}}(\bar{a})\right) \in G_{i}$.

Demonstração.

1. Fixe $\bar{x} \in U$. Então, existe um cubo aberto

$$
B=] p_{1}, q_{1}[\times \cdots \times] p_{n}, q_{n}[
$$

tal que $\bar{x} \in B \subseteq U$. Logo, $p_{i}<x_{i}<q_{i}$.

Sejam $m=\min \left\{q_{i}-x_{i}, x_{i}-p_{i}\right\}$ e $\eta=m / 2$. Então, tomando $V$ como

$$
] p_{1}, q_{1}[\times \cdots \times] x_{i}-\eta, x_{i}+\eta[\times \cdots \times] p_{n}, q_{n}[,
$$

segue o resultado.

2. De $G r(\hat{f}) \in C h(\mathcal{S})$ temos que, $G r\left(\hat{g}_{i}\right) \in C h(\mathcal{S})$. Consequentemente, $G_{i} \in C h(\mathcal{S})$.

3. Seja $(\bar{a}, b) \in \overline{G_{i}}$. Vejamos que $(\bar{a}, 0, b) \in \overline{G r\left(\hat{g}_{i}\right)}$.

Considere $B$ um cubo aberto, com $\bar{a} \in B, r>0$ e $I$ um intervalo aberto, com $b \in I$.

Uma vez que $B \times I$ é um aberto contendo $(\bar{a}, b)$ e $(\bar{a}, b) \in \overline{G_{i}}$, então, existe $\left(\bar{a}^{\prime}, b^{\prime}\right) \in$ $(B \times I) \cap G_{i}$.

Logo,

$$
\left(\bar{a}^{\prime}, 0, b^{\prime}\right) \in \overline{G r\left(\hat{g}_{i}\right)} .
$$

Visto que $B \times]-r, r\left[\times I\right.$ é um aberto ao qual $\left(\bar{a}^{\prime}, 0, b^{\prime}\right)$ pertence, então

$$
(B \times]-r, r[\times I) \cap G r\left(\hat{g}_{i}\right) \neq \emptyset .
$$


4. Fixe $\bar{a} \in U$. Vejamos que $\left(\bar{a}, 0, \frac{\partial \hat{f}}{\partial x_{i}}(\bar{a})\right) \in \overline{G r\left(\hat{g}_{i}\right)}$.

Com efeito, pelo item 1, existem um cubo aberto $V \subseteq U$, tal que $\bar{a} \in V$, e um $\eta>0$ de sorte que $\hat{g}_{i}$ está definida em $V \times(]-\eta, \eta[\backslash\{0\})$ como pseudofunção.

Seja $B \times]-\delta, \delta[\times] \alpha, \beta\left[\right.$ uma vizinhança aberta arbitrária de $(\bar{a}, 0, L), \operatorname{com} L=\frac{\partial \hat{f}}{\partial x_{i}}(\bar{a}) \mathrm{e}$ $B \subseteq U$ um cubo aberto.

Considere $r>0$ tal que

$$
] L-r, L+r[\subseteq] \alpha, \beta[
$$

Logo, pela definição de $L$, temos que existem $V_{1} \subseteq U$ um cubo aberto, tal que $\bar{a} \in V_{1}$ e $\hat{f}$ restrita a $V_{1}$ é função, e $\theta>0$ de modo que para qualquer $\left.\varepsilon \in\right]-\theta, \theta[\backslash\{0\}$, vale

$$
\hat{g}_{i}(\bar{a}, \varepsilon)=\frac{\hat{f}\left(\bar{a}+\bar{\varepsilon}_{i}\right)-\hat{f}(\bar{a})}{\varepsilon} \in(L-r, L+r)
$$

Sejam $W=B \cap V \cap V_{1}$ e $\lambda=\min \{\theta, \eta, \delta\}$.

Tome $\left(\bar{a}, \frac{\lambda}{2}\right) \in W \times(]-\lambda, \lambda[\backslash\{0\})$. Então,

$$
\left.\hat{g}_{i}\left(\bar{a}, \frac{\lambda}{2}\right) \in\right] L-r, L+r[\subseteq] \alpha, \beta[
$$

Portanto,

$$
\left(\bar{a}, \frac{\lambda}{2}, \hat{g}_{i}\left(\bar{a}, \frac{\lambda}{2}\right)\right) \in(B \times]-\delta, \delta[\times] \alpha, \beta[) \cap G r\left(\hat{g}_{i}\right)
$$

Esta última parte do Lema 2.3 .8 pode ser reescrita como $G r\left(\frac{\partial \hat{f}}{\partial x_{i}}\right) \subseteq G_{i}$. A inclusão $G_{i} \subseteq G r\left(\frac{\partial \hat{f}}{\partial x_{i}}\right)$ é válida para o caso de $\hat{f}$ ser uma função diferenciável com derivada contínua. Portanto, sob esta condição e visto que $G_{i} \in C h(\mathcal{S})$, concluímos que $C h(\mathcal{S})$ é fechado sob derivação parcial. É o que afirma o lema seguinte.

Lema 2.3.9 (Fecho sob diferenciação). Se $\hat{f}$ é uma função diferenciável com derivada contínua, então $\operatorname{Gr}\left(\frac{\partial \hat{f}}{\partial x_{i}}\right)=G_{i}$, para $i=1, \ldots, n$ e, portanto, $\operatorname{Gr}\left(\frac{\partial \hat{f}}{\partial x_{i}}\right) \in C h(\mathcal{S})$. 
Demonstração. Fixemos $i \in\{1, \ldots, n\}$.

O caso $\operatorname{Gr}\left(\frac{\partial \hat{f}}{\partial x_{i}}\right) \subseteq G_{i}$ segue diretamente do Lema 2.3.8.

Vejamos, então, que $G_{i} \subseteq G r\left(\frac{\partial \hat{f}}{\partial x_{i}}\right)$.

Para isso, basta mostrarmos que se $\bar{a} \in U$ e $r>0$, então existe um cubo aberto $B \subseteq \mathbb{R}^{n+1}$, $\operatorname{com}(\bar{a}, 0) \in B$, tal que

(†) $\quad$ para todo $(\bar{x}, \varepsilon) \in B, \quad(\bar{x}, \varepsilon, y) \in G r\left(\hat{g}_{i}\right)$ implica $\left|y-\frac{\partial \hat{f}}{\partial x_{i}}(\bar{a})\right|<r$.

Fixemos $\bar{a} \in U$ e $r>0$.

Por hipótese, $\frac{\partial \hat{f}}{\partial x_{i}}(\bar{a})$ existe. Logo, por definição, existem $\gamma>0$ e um cubo aberto $V \subseteq U$, com $\bar{a} \in V$, tal que $\hat{f}$ é função em $V$ e tal que para qualquer $\varepsilon \in]-\gamma, \gamma[\backslash\{0\}$, tem-se

$$
\bar{a}+\bar{\varepsilon}_{i} \in V \text { e }\left|\frac{\hat{f}\left(\bar{a}+\bar{\varepsilon}_{i}\right)-\hat{f}(\bar{a})}{\varepsilon}-\frac{\partial \hat{f}}{\partial x_{i}}(\bar{a})\right|<r .
$$

Ainda por hipótese, $\frac{\partial \hat{f}}{\partial x_{i}}$ é contínua em $\bar{a}$. Logo, existe $W \subseteq U$ um cubo aberto, com $\bar{a} \in W$, tal que para todo $\bar{x} \in W$,

$$
\left|\frac{\partial \hat{f}}{\partial x_{i}}(\bar{x})-\frac{\partial \hat{f}}{\partial x_{i}}(\bar{a})\right|<r .
$$

Considere o cubo aberto $W^{*}=V \cap W$. Podemos escrever

$$
\left.W^{*}=\right] p_{1}, q_{1}[\times \cdots \times] p_{n}, q_{n}[
$$

Como $\bar{a} \in W^{*}$, então $p_{j}<a_{j}<q_{j}$, para $j=1, \ldots, n$.

Considere $m_{i}=\min \left\{q_{i}-a_{i}, a_{i}-p_{i}\right\}$.

Se $m_{i}>\gamma$, então tomamos

$$
B=] p_{1}, q_{1}[\times \cdots \times] a_{i}-\left(m_{i}-\gamma\right), a_{i}+\left(m_{i}-\gamma\right)[\times \cdots \times] p_{n}, q_{n}[\times]-\gamma, \gamma[
$$


Se $m_{i} \leq \gamma$, então tomamos

$$
B=] p_{1}, q_{1}[\times \cdots \times] a_{i}-\frac{m_{i}}{2}, a_{i}+\frac{m_{i}}{2}[\times \cdots \times] p_{n}, q_{n}[\times]-\frac{m_{i}}{2}, \frac{m_{i}}{2}[.
$$

Em ambos os casos, segue que

$$
\bar{x}+\bar{\varepsilon}_{i} \in W^{*} \text {, para todo }(\bar{x}, \varepsilon) \in B .
$$

Seja $(\bar{x}, \varepsilon) \in B$ e suponha, sem perda de generalidade, que $\varepsilon>0$.

Assim, se $(\bar{x}, \varepsilon, y) \in G r\left(\hat{g}_{i}\right)$, então

$$
y=\frac{\hat{f}\left(\bar{x}+\bar{\varepsilon}_{i}\right)-\hat{f}(\bar{x})}{\varepsilon} .
$$

Denotemos por $\check{f}: I \rightarrow \mathbb{R}$ a função

$$
\check{f}(t)=\hat{f}\left(x_{1}, \ldots, x_{i-1}, x_{i}+t, x_{i+1}, \ldots, x_{n}\right),
$$

sendo que $I$ é um intervalo aberto que contém $[0, \varepsilon]$.

Como $\tilde{f}$ é composição de duas funções diferenciáveis, então é diferenciável. Assim, pelo Teorema do Valor Médio, existe $\eta \in] 0, \varepsilon[$ tal que

$$
\frac{d \check{f}}{d t}(\eta)=\frac{\hat{f}\left(\bar{x}+\bar{\varepsilon}_{i}\right)-\hat{f}(\bar{x})}{\varepsilon}=y .
$$

Mas,

$$
\frac{d \check{f}}{d t}(\eta)=\frac{\partial \hat{f}}{\partial x_{i}}\left(\bar{x}+\bar{\eta}_{i}\right) .
$$

Logo,

$$
y=\frac{\partial \hat{f}}{\partial x_{i}}\left(\bar{x}+\bar{\eta}_{i}\right) .
$$


Uma vez que $\bar{x}+\bar{\eta}_{i} \in W^{*}$, então

$$
\left|\frac{\partial \hat{f}}{\partial x_{i}}\left(\bar{x}+\bar{\eta}_{i}\right)-\frac{\partial \hat{f}}{\partial x_{i}}(\bar{a})\right|<r
$$

ou seja,

$$
\left|y-\frac{\partial \hat{f}}{\partial x_{i}}(\bar{a})\right|<r
$$

Considere, agora, os subconjuntos de $\mathbb{R}^{n}$

$$
\begin{aligned}
C_{i}=\{\bar{x} & \in \mathbb{R}^{n}: \exists y_{1} \exists y_{2}\left[y_{1} \neq y_{2} \wedge\left(\bar{x}, y_{1}\right) \in G r(\hat{f}) \wedge\left(\bar{x}, y_{2}\right) \in G r(\hat{f})\right] \\
& \left.\vee(\bar{x},+\infty) \in G_{i} \vee(\bar{x},-\infty) \in G_{i} \vee \exists y_{1} \exists y_{2}\left[y_{1} \neq y_{2} \wedge\left(\bar{x}, y_{1}\right) \in G_{i} \wedge\left(\bar{x}, y_{2}\right) \in G_{i}\right]\right\}
\end{aligned}
$$

e

$$
C=\overline{\bigcup_{i=1}^{n} C_{i}}
$$

Nossa meta é mostrar que $C$ contém todos os pontos nos quais $\hat{f}$ não é uma função $\mathcal{C}^{1}$. Isto será feito no lema a seguir. No lema subsequente, garantimos que $C$ tem interior vazio.

Lema 2.3.10. Denotemos por $f^{*}$ a restrição de $\hat{f}$ ao aberto $U \backslash C$. Então, $f^{*}$ é uma função e as derivadas parciais $\frac{\partial f^{*}}{\partial x_{i}}$ existem e são contínuas, para $i=1, \ldots, n$.

Demonstração. Fixemos $i=1, \ldots, n$.

Vejamos que $f^{*}$ é função.

Suponha, por absurdo, que existam $\bar{a} \in U \backslash C$ e $y_{1}, y_{2} \in \mathbb{R}$, com $y_{1} \neq y_{2}$, tais que $\left(\bar{a}, y_{1}\right),\left(\bar{a}, y_{2}\right) \in G r\left(f^{*}\right)$.

Então, $\left(\bar{a}, y_{1}\right),\left(\bar{a}, y_{2}\right) \in G r(\hat{f})$. Portanto, $\bar{a} \in C_{i} . \quad(\Rightarrow \Leftarrow)$

Vejamos que $\frac{\partial f^{*}}{\partial x_{i}}$ existe.

Suponha, por absurdo, que $\frac{\partial f^{*}}{\partial x_{i}}(\bar{a})$ não exista, para algum $\bar{a} \in U \backslash C$. 
Então, o limite

$$
\lim _{\varepsilon \rightarrow 0} \frac{\hat{f}\left(\bar{a}+\bar{\varepsilon}_{i}\right)-\hat{f}(\bar{a})}{\varepsilon}=\lim _{\varepsilon \rightarrow 0} \hat{g}_{i}(\bar{a}, \varepsilon)
$$

não existe.

Assim, temos dois casos a considerar:

(a) existem sequências $\left(\bar{x}_{j}, \delta_{j}\right)_{j}$ e $\left(\bar{z}_{j}, \theta_{j}\right)_{j}, \operatorname{com} \bar{x}_{j}, \bar{z}_{j} \rightarrow \bar{a}$ e $\delta_{j}, \theta_{j} \rightarrow 0$, tais que

$$
y_{1}=\lim _{j} \hat{g}_{i}\left(\bar{x}_{j}, \delta_{j}\right) \neq \lim _{j} \hat{g}_{i}\left(\bar{z}_{j}, \theta_{j}\right)=y_{2} .
$$

(b) $\lim _{\varepsilon \rightarrow 0} \hat{g}_{i}(\bar{a}, \varepsilon)= \pm \infty$.

No caso (a),

$$
\left(\bar{a}, 0, y_{1}\right),\left(\bar{a}, 0, y_{2}\right) \in \overline{G r\left(\hat{g}_{i}\right)}
$$

Já no caso (b),

$$
(\bar{a}, 0,+\infty) \in \overline{G r\left(\hat{g}_{i}\right)} \text { ou }(\bar{a}, 0,-\infty) \in \overline{G r\left(\hat{g}_{i}\right)}
$$

Em qualquer dos casos, $\bar{a} \in C_{i} . \quad(\Rightarrow \Leftarrow)$

Portanto, $\frac{\partial f^{*}}{\partial x_{i}}$ é uma função definida no aberto $U \backslash C$.

Resta mostrar que $\frac{\partial f^{*}}{\partial x_{i}}$ é contínua.

Antes, note que, pelo Lema 2.3.8, temos

$$
\text { (†) } \overline{G r\left(\frac{\partial f^{*}}{\partial x_{i}}\right)} \subseteq G_{i}
$$

Suponha, por absurdo, que $\frac{\partial f^{*}}{\partial x_{i}}$ seja descontínua num ponto $\bar{a} \in U \backslash C$.

Então, existe $\left(\bar{a}_{j}\right)_{j} \in U \backslash C, \operatorname{com} \bar{a}_{j} \rightarrow \bar{a}$, tal que

$$
\frac{\partial f^{*}}{\partial x_{i}}\left(\bar{a}_{j}\right) \nrightarrow \frac{\partial f^{*}}{\partial x_{i}}(\bar{a}) .
$$

Por conseguinte, existem um intervalo aberto $I, \operatorname{com} \frac{\partial f^{*}}{\partial x_{i}}(\bar{a}) \in I$, e uma subsequência 
$\left(\frac{\partial f^{*}}{\partial x_{i}}\left(\bar{a}_{j_{l}}\right)\right)_{l}$ tal que

$$
\frac{\partial f^{*}}{\partial x_{i}}\left(\bar{a}_{j_{l}}\right) \notin I, \text { para todo } l \text {. }
$$

Assim:

(i) Se $\left(\frac{\partial f^{*}}{\partial x_{i}}\left(\bar{a}_{j_{l}}\right)\right)_{l}$ é limitada, então, por Bolzano-Weierstrass, tem subsequência $\left(\frac{\partial f^{*}}{\partial x_{i}}\left(\bar{a}_{j_{k}}\right)\right)_{k}$ convergente.

Seja $\lim _{k} \frac{\partial f^{*}}{\partial x_{i}}\left(\bar{a}_{j_{k}}\right)=b$. Logo,

$$
(\bar{a}, b) \in \overline{G r\left(\frac{\partial f^{*}}{\partial x_{i}}\right)} .
$$

Por $(\dagger)$, temos que

$$
(\bar{a}, b),\left(\bar{a}, \frac{\partial f^{*}}{\partial x_{i}}(\bar{a})\right) \in G_{i} .
$$

Também, como $\frac{\partial f^{*}}{\partial x_{i}}\left(\bar{a}_{j_{k}}\right) \nrightarrow \frac{\partial f^{*}}{\partial x_{i}}(\bar{a})$, então

$$
b \neq \frac{\partial f^{*}}{\partial x_{i}}(\bar{a})
$$

Portanto, $\bar{a} \in C_{i} . \quad(\Rightarrow \Leftarrow)$

(ii) Se $\left(\frac{\partial f^{*}}{\partial x_{i}}\left(\bar{a}_{j_{l}}\right)\right)_{l}$ é ilimitada, então tem subsequência $\left(\frac{\partial f^{*}}{\partial x_{i}}\left(\bar{a}_{j_{k}}\right)\right)_{k}$ tal que

$$
\frac{\partial f^{*}}{\partial x_{i}}\left(\bar{a}_{j_{k}}\right) \rightarrow \pm \infty
$$

Logo,

$$
(\bar{a}, \pm \infty) \in \overline{G r\left(\frac{\partial f^{*}}{\partial x_{i}}\right)}
$$

Por $(\dagger)$, obtemos $(\bar{a}, \pm \infty) \in G_{i}$ e, assim, $\bar{a} \in C_{i} . \quad(\Rightarrow \Leftarrow)$

Lema 2.3.11. C tem interior vazio. 
Demonstração. Suponha, por absurdo, que $C$ tenha um ponto interior. Então, pelo Corolário 2.1.18, $\bigcup_{i=1}^{n} C_{i}$ tem medida não nula. Logo, existe $i \in\{1, \ldots, n\}$ tal que $C_{i}$ tem medida não nula. Novamente, pelo Corolário 2.1.18, $C_{i}$ tem um ponto interior. Então, existe um cubo aberto $B$ contido em $C_{i}$. Como $C_{i} \subseteq \bar{U}$, então $B$ pode ser reduzido de tal modo que $B \subseteq U$. Assim, pelo Lema 2.3.1, existem um cubo $B^{\prime} \subseteq B$ e uma função contínua $f: B^{\prime} \rightarrow \mathbb{R}$ tal que $G r(f) \in C h(\mathcal{S})_{n+1}$ e $G r(f) \subseteq G r(\hat{f})$. Dessa forma, podemos assumir que $B$ tenha sido reduzido a um cubo aberto no qual $\hat{f}$ é uma função contínua. Passaremos, então, a denotar $\hat{f}$ por $f$. Como consequência, a função

$$
\hat{g}_{i}\left(\bar{x}, \varepsilon_{i}\right)=\frac{f\left(\bar{x}+\bar{\varepsilon}_{i}\right)-f(\bar{x})}{\varepsilon},
$$

restrita a $B \times(]-\lambda, \lambda[\backslash\{0\})$, com $\lambda>0$, é uma função contínua.

Denotaremos $\hat{g}_{i} \uparrow B \times(]-\lambda, \lambda[\backslash\{0\})$ por $g$. Note que,

$$
\begin{aligned}
\operatorname{Gr}(g)=\left\{(\bar{x}, \varepsilon, y) \in \mathbb{R}^{n+2}:\right. & \bar{x} \\
& \in B \wedge \varepsilon \in]-\lambda, \lambda[\wedge \varepsilon \neq 0 \\
& \left.\wedge \exists z_{1} \exists z_{2}\left(\left(\bar{x}, z_{1}\right) \in G r(f) \wedge\left(\bar{x}+\bar{\varepsilon}_{i}, z_{2}\right) \in G r(f) \wedge \varepsilon y=z_{2}-z_{1}\right)\right\}
\end{aligned}
$$

ou seja, $\operatorname{Gr}(g)$ é definido por uma $C h$-fórmula. Portanto, $G r(g) \in C h(\mathcal{S})_{n+2}$.

Indiquemos $g \uparrow B \times] 0, \lambda\left[\right.$ por $g^{+}$e definamos $\left.g^{-}: B \times\right] 0, \lambda[\rightarrow \mathbb{R}$ por

$$
g^{-}(\bar{x}, \varepsilon)=g(\bar{x},-\varepsilon)
$$

Sejam

$$
\begin{aligned}
G & =\{(\bar{x}, y):(\bar{x}, 0, y) \in \overline{G r(g)}\} \\
G^{+} & =\left\{(\bar{x}, y):(\bar{x}, 0, y) \in \overline{G r\left(g^{+}\right)}\right\}
\end{aligned}
$$

e

$$
G^{-}=\left\{(\bar{x}, y):(\bar{x}, 0, y) \in \overline{G r\left(g^{-}\right)}\right\}
$$


Considere, ainda,

$$
S_{g^{+}}=\left\{\bar{x}: \exists y_{1} \exists y_{2} \in \mathbb{R}_{ \pm \infty}\left(y_{1} \neq y_{2} \wedge\left(\bar{x}, 0, y_{1}\right) \in \overline{G r\left(g^{+}\right)} \wedge\left(\bar{x}, 0, y_{2}\right) \in \overline{G r\left(g^{+}\right)}\right)\right\}
$$

e

$$
S_{g^{-}}=\left\{\bar{x}: \exists y_{1} \exists y_{2} \in \mathbb{R}_{ \pm \infty}\left(y_{1} \neq y_{2} \wedge\left(\bar{x}, 0, y_{1}\right) \in \overline{G r\left(g^{-}\right)} \wedge\left(\bar{x}, 0, y_{2}\right) \in \overline{G r\left(g^{-}\right)}\right)\right\}
$$

$\operatorname{com} \mathbb{R}_{ \pm \infty}=\mathbb{R} \cup\{ \pm \infty\}$.

Então, pelo Lema 2.3.3, $S_{g^{+}}$e $S_{g^{-}}$têm interior vazio. Uma vez que $S_{g^{+}}, S_{g^{-}} \in C h(\mathcal{S})$, então, pelo Corolário 2.1.19,

$$
\widehat{B \backslash S_{g^{+}}} \neq \emptyset \neq \widehat{B \backslash S_{g^{-}}}
$$

De $\widehat{B \backslash S_{g^{+}}} \neq \emptyset$, temos que existe um cubo aberto $B_{1}$ contido em $B \backslash S_{g^{+}}$. Como $B_{1} \nsubseteq S_{g^{-}}$, então existe $\bar{b}_{1} \in B_{1}$ tal que $\bar{b}_{1} \notin S_{g^{-}}$. Seja

$$
m=\min \left\{d\left(\bar{b}_{1}, S_{g^{-}}\right), d\left(\bar{b}_{1}, S_{g^{+}}\right)\right\}
$$

em que $d(\bar{x}, \mathcal{D})$ é a distância entre o ponto $\bar{x}$ e o conjunto $\mathcal{D}$.

Seja $B\left(\bar{b}_{1} ; m\right)=\left\{\bar{x} \in B:\left\|\bar{x}-\bar{b}_{1}\right\|<m\right\}$. Então,

$$
B\left(\bar{b}_{1} ; m\right) \subseteq B \backslash\left(S_{g^{-}} \cup S_{g^{+}}\right)
$$

e, portanto

$$
B\left(\bar{b}_{1} ; m\right) \cap\left(S_{g^{-}} \cup S_{g^{+}}\right)=\emptyset .
$$

Assim, podemos assumir que $B$ tenha sido reduzido de tal forma que $B \cap\left(S_{g^{-}} \cup S_{g^{+}}\right)=\emptyset$.

Portanto, para todo $\bar{x} \in B$ valem:

$\left(1^{*}\right)$ Existe um único $y \in \mathbb{R} \cup\{ \pm \infty\}$ tal que $(\bar{x}, y) \in G^{+}$. Chamemos tal $y$ de $y^{+}(\bar{x})$.

$\left(2^{*}\right)$ Existe um único $y \in \mathbb{R} \cup\{ \pm \infty\}$ tal que $(\bar{x}, y) \in G^{-}$. Chamemos este $y$ de $y^{-}(\bar{x})$. 
Agora, considere os seguintes conjuntos:

$$
\begin{aligned}
& U_{1}=\left\{\bar{x} \in B: y^{+}(\bar{x})=+\infty=y^{-}(\bar{x})\right\}, \\
& U_{2}=\left\{\bar{x} \in B: y^{+}(\bar{x})=-\infty=y^{-}(\bar{x})\right\},
\end{aligned}
$$

e

$$
U_{3}=\left\{\bar{x} \in B: y^{+}(\bar{x}) \neq y^{-}(\bar{x})\right\}
$$

Então,

(i) $G=G^{+} \cup G^{-}$.

Com efeito, como $\operatorname{Gr}\left(g^{+}\right) \subseteq G r(g)$, então

$$
\overline{G r\left(g^{+}\right)} \subseteq \overline{G r(g)}
$$

Assim,

$$
G^{+} \subseteq G
$$

Agora, seja $(\bar{x}, y) \in G^{-}$. Então, $(\bar{x}, 0, y) \in \overline{G r\left(g^{-}\right)}$. Logo, existe uma sequência $\left(\bar{x}_{n}, \varepsilon_{n}, y_{n}\right)_{n}$ em $\operatorname{Gr}\left(g^{-}\right)$tal que $\left(\bar{x}_{n}, \varepsilon_{n}, y_{n}\right) \rightarrow(\bar{x}, 0, y)$. Portanto, $\left(\bar{x}_{n},-\varepsilon_{n}, y_{n}\right)_{n} \in G r(g)$ e, além disso, $\left(\bar{x}_{n},-\varepsilon_{n}, y_{n}\right) \rightarrow(\bar{x}, 0, y)$. Assim, $(\bar{x}, 0, y) \in \overline{G r(g)}$ e, portanto, $(\bar{x}, y) \in G$.

Em resumo, $G^{+} \cup G^{-} \subseteq G$.

Inversamente, seja $(\bar{x}, y) \in G$. Então, $(\bar{x}, 0, y) \in \overline{G r(g)}$. Logo, existe uma sequência $\left(\bar{x}_{j}, \varepsilon_{j}, y_{j}\right)_{j}$ em $\operatorname{Gr}(g)$ tal que $\left(\bar{x}_{j}, \varepsilon_{j}, y_{j}\right) \rightarrow(\bar{x}, 0, y)$.

Seja $J=\left\{j: \varepsilon_{j} \in\right] 0, \lambda[\}$.

Então, $|J| \leq \omega$.

Se $|J| \in \omega$, então existe $j^{\prime}=\max J$. Dessa forma, $\left(\varepsilon_{j}\right)_{j>j^{\prime}}$ é uma subsequência de $\left(\varepsilon_{j}\right)_{j}$, tal que

$$
\left.\varepsilon_{j} \in\right]-\lambda, 0\left[\text {, para todo } j>j^{\prime}\right. \text {. }
$$


Portanto,

$$
\left(\bar{x}_{j},-\varepsilon_{j}, g^{-}\left(\bar{x}_{j},-\varepsilon_{j}\right)\right)=\left(\bar{x}_{j},-\varepsilon_{j}, g\left(\bar{x}_{j}, \varepsilon_{j}\right)\right)=\left(\bar{x}_{j},-\varepsilon_{j}, y_{j}\right), \text { para todo } j>j^{\prime}
$$

Logo,

$$
\left(\bar{x}_{j},-\varepsilon_{j}, y_{j}\right)_{j>j^{\prime}} \in G r\left(g^{-}\right)
$$

e, além disso

$$
\left(\bar{x}_{j},-\varepsilon_{j}, y_{j}\right) \rightarrow(\bar{x}, 0, y)
$$

Assim,

$$
(\bar{x}, 0, y) \in \overline{G r\left(g^{-}\right)}
$$

o que implica

$$
(\bar{x}, 0, y) \in G^{-}
$$

Se $|J|=\omega$, então $(\bar{x}, 0, y) \in \overline{G r\left(g^{+}\right)}$. Ou seja, $(\bar{x}, 0, y) \in G^{+}$.

(ii) $B=U_{1} \cup U_{2} \cup U_{3}$.

Seja $\bar{x} \in B$ tal que $\bar{x} \notin U_{2}$ e $\bar{x} \notin U_{3}$. Então,

$$
y^{+}(\bar{x})=y^{-}(\bar{x})=y \text { e } y \neq-\infty \text {. }
$$

Logo, $y \in \mathbb{R}$ ou $y=+\infty$.

Suponha $y \in \mathbb{R}$. Pela definição de $y$ e pelo item $(i),(\bar{x}, y) \in G$.

Vejamos que este $y$ é único.

De fato, suponha, por absurdo, que exista $y^{*} \in \mathbb{R} \backslash\{y\}$ tal que $\left(\bar{x}, y^{*}\right) \in G$. Então, pelo item (i), $\left(\bar{x}, y^{*}\right) \in G^{+}$ou $\left(\bar{x}, y^{*}\right) \in G^{-}$.

Se $\left(\bar{x}, y^{*}\right) \in G^{-}$, então, uma vez que $(\bar{x}, y) \in G^{-}$, temos, por $\left(2^{*}\right)$, que $y=y^{*} .(\Rightarrow \Leftarrow)$

Analogamente, se $\left(\bar{x}, y^{*}\right) \in G^{+}$, então, uma vez que $(\bar{x}, y) \in G^{+}$, temos, por $\left(1^{*}\right)$, que $y=y^{*} .(\Rightarrow \Leftarrow)$ 
Assim, existe um único $y \in \mathbb{R}$ tal que $(\bar{x}, y) \in G\left(=G_{i}\right)$.

Portanto, pela definição de $C_{i},(\bar{x}, y) \notin C_{i}$. Contradição, pois $B \subseteq C_{i}$.

Assim, $y=+\infty$.

Para completarmos a demonstração, mostraremos que $U_{1}, U_{2}$ e $U_{3}$ têm interior vazio.

AFIRMAÇÃO 1. U tem interior vazio.

Prova da Afirmação. Suponha, por absurdo, que exista um cubo aberto $B_{1}$ contido em $U_{1}$.

Sejam $\bar{a}, \bar{a}+\bar{\eta}_{i} \in B_{1}$, para algum $\eta>0$, e $L$ o segmento (em $\mathbb{R}^{n+1}$ ) que liga o ponto $(\bar{a}, f(\bar{a}))$ ao ponto $\left(\bar{a}+\bar{\eta}_{i}, f\left(\bar{a}+\bar{\eta}_{i}\right)\right)$.

Então, não existe um segmento não degenerado $I \subseteq L$, com um dos extremos igual a $(\bar{a}, f(\bar{a}))$ e tal que $I \subseteq G r(f)$.

Suponha, por absurdo, que exista tal segmento $I$. Então, existe $0<s \leq 1$ tal que

$$
I=\left\{(\bar{x}, f(\bar{x})): \bar{x}=(1-t) \bar{a}+t\left(\bar{a}+\bar{\eta}_{i}\right) \text { e } f(\bar{x})=(1-t) f(\bar{a})+t f\left(\bar{a}+\bar{\eta}_{i}\right), t \in[0, s]\right\} .
$$

Vejamos, então, que para todo $t \in(0, s), g^{+}(\bar{a}, t \eta)=g^{+}(\bar{a}, \eta)$.

De fato, seja $t \in] 0, s[$. Então,

$$
\bar{x}=(1-t) \bar{a}+t\left(\bar{a}+\bar{\eta}_{i}\right)=\bar{a}+t \bar{\eta}_{i} \text { e } f(\bar{x})=(1-t) f(\bar{a})+t f\left(\bar{a}+\bar{\eta}_{i}\right) .
$$

Logo,

$$
f\left(\bar{a}+t \bar{\eta}_{i}\right)-f(\bar{a})=t\left[f\left(\bar{a}+\bar{\eta}_{i}\right)-f(\bar{a})\right]
$$

ou seja

$$
\frac{f\left(\bar{a}+t \bar{\eta}_{i}\right)-f(\bar{a})}{t \eta}=\frac{f\left(\bar{a}+\bar{\eta}_{i}\right)-f(\bar{a})}{\eta} .
$$

Uma vez que, $\overline{t \eta}_{i}=t \bar{\eta}_{i}$, temos $g^{+}(\bar{a}, t \eta)=g^{+}(\bar{a}, \eta)$.

Agora, tome a sequência $\left.\left(\frac{s}{j}\right)_{j} \in\right] 0, s\left[\right.$. Então, $\frac{\eta s}{j} \rightarrow 0$. Além disso, $g^{+}\left(\bar{a}, \frac{s}{j} \eta\right)=g^{+}(\bar{a}, \eta)$, 
para todo $j$. Assim, $g^{+}\left(\bar{a}, \frac{s}{j} \eta\right) \rightarrow g^{+}(\bar{a}, \eta)$.

Portanto, $\left(\bar{a}, \frac{\eta s}{j}, g^{+}\left(\bar{a}, \frac{s \eta}{j}\right)\right) \in G r\left(g^{+}\right)$tem o ponto $\left(\bar{a}, 0, g^{+}(\bar{a}, \eta)\right)$ como limite, o que implica que $\left(\bar{a}, g^{+}(\bar{a}, \eta)\right) \in G^{+}$.

Entretanto, de $\bar{a} \in B_{1} \subseteq U_{1}$, temos que $y^{+}(\bar{a})=+\infty$. Assim, por $\left(1^{*}\right), g^{+}(\bar{a}, \eta)=+\infty$. Contradição, pois $g^{+}(\bar{a}, \eta) \in \mathbb{R}$.

Portanto, não existe tal segmento $I$.

Como $L$ é afim, então, por (EF5) para $G r(f)$, temos que $G r(f) \cap L$ tem $N \geq 1$ componentes conexas $A_{1}, \ldots, A_{N}$.

Suponha, sem perda de generalidade, que $(\bar{a}, f(\bar{a})) \in A_{1}$. Como não pode haver um segmento contido em $L \cap G r(f)$, com extremo igual a $(\bar{a}, f(\bar{a}))$, então $A_{1}$ não pode ser um segmento. Portanto, $A_{1}=\{(\bar{a}, f(\bar{a}))\}$.

A componente $A_{2}$ é dada por

$$
A_{2}=\left(\bar{a}+\bar{\mu}_{i}, f\left(\bar{a}+\bar{\mu}_{i}\right)\right) \cdot(1-t)+\left(\bar{a}+\bar{\eta}_{i}, f\left(\bar{a}+\bar{\eta}_{i}\right)\right) \cdot t
$$

sendo que $0 \leq t<1$ e $\eta>\mu>0$, já que $A_{1} \cap A_{2}=\emptyset$.

Segue, portanto, que reduzindo $\eta$ a $\mu$, podemos assumir que

(†) não existem pontos em $G r(f) \cap L$ diferentes dos extremos de $L$.

Para cada $\bar{x} \in \pi_{n+1, n}[L]$, denotemos por $l(\bar{x}) \in \mathbb{R}$ a coordenada tal que $(\bar{x}, l(\bar{x})) \in L$.

Vejamos que existem $\delta \in(0, \eta)$ e $\gamma \in(\delta, \eta)$, de sorte que

$$
f\left(\bar{a}+\bar{\gamma}_{i}\right)<l\left(\bar{a}+\bar{\gamma}_{i}\right) \text { e } l\left(\bar{a}+\bar{\delta}_{i}\right)<f\left(\bar{a}+\bar{\delta}_{i}\right)
$$

Suponha $f\left(\bar{a}+\bar{\eta}_{i}\right)-f(\bar{a})>0$. 
Da implicação $(\bar{a}, y) \in G^{+} \Rightarrow y=+\infty$, temos que $\lim _{\varepsilon \rightarrow 0^{+}} g(\bar{a}, \varepsilon)=+\infty$. Portanto,

$$
\lim _{\varepsilon \rightarrow 0^{+}} \frac{f\left(\bar{a}+\bar{\varepsilon}_{i}\right)-f(\bar{a})}{\varepsilon}=+\infty
$$

Então, pela definição de limite, temos que para $\eta^{-1}\left[f\left(\bar{a}+\bar{\eta}_{i}\right)-f(\bar{a})\right]>0$, existe $s_{1}>0$ tal que

$$
f\left(\bar{a}+\bar{\varepsilon}_{i}\right)-f(\bar{a})>\frac{\varepsilon}{\eta}\left[f\left(\bar{a}+\bar{\eta}_{i}\right)-f(\bar{a})\right]
$$

para $0<\varepsilon<s_{1}$.

Seja $s_{1}^{*}=\min \left\{\eta, s_{1}\right\}$ e tome $\delta=\frac{s_{1}^{*}}{2}$.

Portanto,

$f\left(\bar{a}+\bar{\delta}_{i}\right)-f(\bar{a})>t\left[f\left(\bar{a}+\bar{\eta}_{i}\right)-f(\bar{a})\right] \Leftrightarrow f\left(\bar{a}+\bar{\delta}_{i}\right)>(1-t) f(\bar{a})+t f\left(\bar{a}+\bar{\eta}_{i}\right)=l\left(\bar{a}+t \bar{\eta}_{i}\right)=l\left(\bar{a}+\bar{\delta}_{i}\right)$, $\left.\operatorname{com} t=\frac{\delta}{\eta} \in\right] 0,1[$.

Analogamente, de $\left(\bar{a}+\bar{\eta}_{i}, y\right) \in G^{-} \Rightarrow y=+\infty$, segue que

$$
\lim _{\varepsilon \rightarrow 0^{-}} \frac{f\left(\bar{a}+\bar{\eta}_{i}+\bar{\varepsilon}_{i}\right)-f\left(\bar{a}+\bar{\eta}_{i}\right)}{\varepsilon}=+\infty
$$

Então, para $\eta^{-1}\left[f\left(\bar{a}+\bar{\eta}_{i}\right)-f(\bar{a})\right]>0$, existe $s_{2}>0$ tal que

$$
\frac{f\left(\bar{a}+\bar{\eta}_{i}+\bar{\varepsilon}_{i}\right)-f\left(\bar{a}+\bar{\eta}_{i}\right)}{\varepsilon}>\frac{f\left(\bar{a}+\bar{\eta}_{i}\right)-f(\bar{a})}{\eta},
$$

para todo $\varepsilon \in]-s_{2}, 0[$.

Considere $s_{2}^{*}=\min \left\{s_{2}, \eta-\delta\right\}$ e tome $\left.\delta^{*}=-\frac{s_{2}^{*}}{2} \in\right]-s_{2}, 0[$. Assim,

$$
\frac{f\left(\bar{a}+\bar{\eta}_{i}+\bar{\delta}^{*}{ }_{i}\right)-f\left(\bar{a}+\bar{\eta}_{i}\right)}{\delta^{*}}>\frac{f\left(\bar{a}+\bar{\eta}_{i}\right)-f(\bar{a})}{\eta},
$$


que é equivalente a

$$
f\left(\bar{a}+{\overline{\left(\eta+\delta^{*}\right)}}_{i}\right)<\left(1+\frac{\delta^{*}}{\eta}\right) f\left(\bar{a}+\bar{\eta}_{i}\right)-\frac{\delta^{*}}{\eta} f(\bar{a}) .
$$

Note que, $0<\eta+\delta^{*}<\eta$. Consequentemente, $\left.t=1+\frac{\delta^{*}}{\eta} \in\right] 0,1[$. Podemos, portanto, reescrever o membro direito da desigualdade acima como

$$
\left(1+\frac{\delta^{*}}{\eta}\right) f\left(\bar{a}+\bar{\eta}_{i}\right)-\frac{\delta^{*}}{\eta} f(\bar{a})=t f\left(\bar{a}+\bar{\eta}_{i}\right)+(1-t) f(\bar{a})=l\left(\bar{a}+\bar{\eta}_{i}\right)=l\left(\bar{a}+{\overline{\left(\eta+\delta^{*}\right.}}_{i}\right) .
$$

Ao tomar $\gamma=\eta+\delta^{*}>\delta$, concluímos que

$$
f\left(\bar{a}+\bar{\gamma}_{i}\right)=f\left(\bar{a}+{\overline{\left(\eta+\delta^{*}\right)_{i}}}\right)<l\left(\bar{a}+{\overline{\left(\eta+\delta^{*}\right.}}_{i}\right)=l\left(\bar{a}+\bar{\gamma}_{i}\right) .
$$

O caso $f\left(\bar{a}+\bar{\eta}_{i}\right)-f(\bar{a})<0$ é análogo.

Agora, uma vez que $f$ é contínua, segue do Teorema do Valor Intermediário, que existe $\varepsilon \in] \delta, \gamma\left[\right.$ de sorte que $f\left(\bar{a}+\bar{\varepsilon}_{i}\right)=l\left(\bar{a}+\bar{\varepsilon}_{i}\right)$, o que contradiz $(\dagger)$.

AFIRMAÇÃO 2. $U_{2}$ não tem ponto interior.

Prova da Afirmação. A demonstração é análoga ao caso anterior.

AFIRMAÇÃO 3. $U_{3}$ não tem ponto interior.

Prova da Afirmação. Suponha, por contradição, que exista um cubo aberto $B_{3} \subseteq U_{3}$.

Seja $\bar{a} \in B_{3}$ e sejam $b=y^{+}(\bar{a})$ e $c=y^{-}(\bar{a})$. Assim,

$$
b=\lim _{\varepsilon \rightarrow 0} g^{+}(\bar{a}, \varepsilon)=\lim _{\varepsilon \rightarrow 0^{+}} g(\bar{a}, \varepsilon)
$$

e

$$
c=\lim _{\varepsilon \rightarrow 0} g^{-}(\bar{a}, \varepsilon)=\lim _{\varepsilon \rightarrow 0^{-}} g(\bar{a}, \varepsilon)
$$


Suponha, sem perda de generalidade, que $b<c$ e seja $d=(c+b) / 2$.

Por definição de limite, segue da primeira igualdade, que para $(c-b) / 2>0$, existe um cubo $\left.B_{3}^{*} \times\right] 0, s^{*}$, sendo que $s^{*}>0$ e $B_{3}^{*} \subseteq B_{3}$ é um cubo de centro $\bar{a}$ e lados de comprimento $2 \beta^{*}>0$ e, além disso,

$$
\left|g^{+}(\bar{x}, \gamma)-b\right|<\frac{c-b}{2}
$$

$\left.\operatorname{para}(\bar{x}, \gamma) \in B_{3}^{*} \times\right] 0, s^{*}[$

Em particular,

$$
g^{+}(\bar{x}, \gamma)<\frac{c-b}{2}+b=d
$$

De maneira análoga, segue, para $(c-b) / 2>0$, que existe um cubo $\left.B_{3}^{* *} \times\right] 0, s^{* *}[$, em que $s^{* *}>0$ e $B_{3}^{* *} \subseteq B_{3}$ é um cubo de centro $\bar{a}$ e lados de comprimento $2 \beta^{* *}>0$ e, também,

$$
\left|g^{-}(\bar{x}, \gamma)-c\right|<\frac{c-b}{2}
$$

para todo $\left.(\bar{x}, \gamma) \in B_{3}^{* *} \times\right] 0, s^{* *}[$.

Particularmente,

$$
d=-\left(\frac{c-b}{2}\right)+c<g^{-}(\bar{x}, \gamma)
$$

Agora, tome $\vartheta=\min \left\{s^{*}, s^{* *}, \beta^{*}, \beta^{* *}\right\}$.

Seja $B_{3}^{\prime}$ o cubo de centro $\bar{a}$ cujos lados têm comprimento $2 \vartheta$.

Portanto, para todo $\left.(\bar{x}, \gamma) \in B_{3}^{\prime} \times\right] 0, \vartheta\left[, g^{+}(\bar{x}, \gamma)<d\right.$ e $d<g^{-}(\bar{x}, \gamma)$. 
Seja $\gamma \in] 0, \vartheta[$. Então,

$$
\begin{aligned}
d>g^{+}(\bar{a}, \gamma) & =\frac{f\left(\bar{a}+\bar{\gamma}_{i}\right)-f(\bar{a})}{\gamma} \\
& =\frac{f\left(\left(\bar{a}+\bar{\gamma}_{i}\right)-\bar{\gamma}_{i}\right)-f\left(\bar{a}+\bar{\gamma}_{i}\right)}{-\gamma} \\
& =g\left(\bar{a}+\bar{\gamma}_{i},-\gamma\right) \\
& =g^{-}\left(\bar{a}+\bar{\gamma}_{i}, \gamma\right)>d,
\end{aligned}
$$

uma vez que $\left.\left(\bar{a}+\bar{\gamma}_{i}, \gamma\right) \in B_{3}^{\prime} \times\right] 0, \vartheta[.(\Rightarrow \Leftarrow)$

Como os conjuntos $U_{1}, U_{2}$ e $U_{3}$ estão em $C h(\mathcal{S})$ e têm interior vazio, então, pelo Corolário 2.1.18, cada um deles tem medida nula. Logo, $U_{1} \cup U_{2} \cup U_{3}$ tem medida nula e, novamente pelo Corolário 2.1.18, $U_{1} \cup U_{2} \cup U_{3}=B$ tem interior vazio. $(\Rightarrow \Leftarrow)$

Definição 2.3.12. Suponha $f: U \rightarrow \mathbb{R}^{m}$, com $U \subseteq \mathbb{R}^{n}$ aberto. Então, dizemos que $f$ é $\mathcal{C}^{1}$ se todas as derivadas parciais $\frac{\partial f}{\partial x_{i}}(i=1, \ldots, n)$ existem e são contínuas.

Seja $p>1$. Dizemos, então, que $f$ é $\mathcal{C}^{p}$ se $f$ é $\mathcal{C}^{1}$ e a diferencial $d f: U \rightarrow \mathbb{R}^{m n}$ é $\mathcal{C}^{p-1}$.

Por convenção, $f$ é dita $\mathcal{C}^{0}$ se $f$ é contínua.

As asserções a seguir são resultados bastante conhecidos. Vamos usá-las para verificar se uma função é $\mathcal{C}^{p}$.

Fato. Seja $f: U \rightarrow \mathbb{R}^{m}$, com $U \subseteq \mathbb{R}^{n}$ aberto. Então, para $p \geq 1$ :

1. $f e^{\prime} \mathcal{C}^{p} \Leftrightarrow \frac{\partial f}{\partial x_{i}}(i=1, \ldots, n)$ existe e é $\mathcal{C}^{p-1}$.

2. $f$ é $\mathcal{C}^{p} \Leftrightarrow f_{j}: U \rightarrow \mathbb{R}(j=1, \ldots, m)$ é $\mathcal{C}^{p}$, de modo que $f=\left(f_{1}, \ldots, f_{m}\right)$.

Teorema 2.3.13. Sejam $m, n \in \mathbb{N}^{+}$e $p \in \mathbb{N}$. Considere $\hat{f}: U \rightarrow \mathbb{R}^{m}$ uma pseudofunção tal que $\operatorname{Gr}(\hat{f}) \in C h(\mathcal{S})_{n+m}$ e $U \subseteq \mathbb{R}^{n}$ é aberto. Então, existe um conjunto fechado $C \in C h(\mathcal{S})_{n}$, com interior vazio, tal que a restrição de $\hat{f}$ a $U \backslash C$ é uma função $\mathcal{C}^{p}$.

Demonstração. Seja $m=1$. 
Vamos proceder por indução em $p$.

Seja $p=1$. Note que este caso cobre o $p=0$.

Tome $C$ como $\overline{\bigcup_{i=1}^{n} C_{i}}$, com

$$
\begin{aligned}
C_{i}=\{\bar{x} & \in \mathbb{R}^{n}: \exists y_{1} \exists y_{2}\left[y_{1} \neq y_{2} \wedge\left(\bar{x}, y_{1}\right) \in G r(\hat{f}) \wedge\left(\bar{x}, y_{2}\right) \in G r(\hat{f})\right] \\
& \left.\vee(\bar{x},+\infty) \in G_{i} \vee(\bar{x},-\infty) \in G_{i} \vee \exists y_{1} \exists y_{2}\left[y_{1} \neq y_{2} \wedge\left(\bar{x}, y_{1}\right) \in G_{i} \wedge\left(\bar{x}, y_{2}\right) \in G_{i}\right]\right\}
\end{aligned}
$$

Pelo Lema 2.3.11, $C$ tem interior vazio. Além disso, chamando de $f^{*}$ a restrição de $\hat{f}$ a $U \backslash C$, temos, pelo Lema 2.3.10, que as derivadas parciais $\frac{\partial f^{*}}{\partial x_{i}}$ 's existem e são contínuas. Ou seja, $f^{*}$ é $\mathcal{C}^{1}$.

Seja $p>1$.

Suponha que a proposição em questão valha para todo $1 \leq q<p$.

Seja $C_{(1)} \in C h(\mathcal{S})$ o conjunto que testemunha $C$ para o caso $m=1, p=1$.

Seja, ainda,

$$
G_{i}=\left\{(\bar{x}, y):(\bar{x}, 0, y) \in \overline{G r\left(\hat{g}_{i}\right)}\right\}
$$

de modo que $\hat{g}_{i}$ é a pseudofunção da Definição 2.3.7.

Então, para cada $i=1, \ldots, n$, definimos:

$$
G r\left(\hat{h}_{i}\right)=G_{i} \cup G_{i}^{\prime}
$$

em que $G_{i}^{\prime}=\left\{(\bar{x}, y):(\bar{x}, \pm \infty) \in G_{i} \wedge y=1\right\}$

Considere, para cada $i$,

$$
D_{i}=\left\{\bar{x}: \exists y_{1} \exists y_{2}\left(y_{1} \neq y_{2} \wedge\left(\bar{x}, y_{1}\right) \in G r\left(\hat{h}_{i}\right) \wedge\left(\bar{x}, y_{2}\right) \in G r\left(\hat{h}_{i}\right)\right)\right\}
$$

Então, $D_{i} \subseteq C_{(1)}$. 
De fato, dado $\bar{x} \in D_{i}$, segue que existem distintos $y_{1}$ e $y_{2}$ tal que $\left(\bar{x}, y_{1}\right),\left(\bar{x}, y_{2}\right) \in G r\left(\hat{h}_{i}\right)$. Temos, então, pela definição de $\hat{h}_{i}$, duas situações a serem consideradas:

(a) $\left(\bar{x}, y_{1}\right),\left(\bar{x}, y_{2}\right) \in G_{i}$.

(b) $\left(\bar{x}, y_{1}\right) \in G_{i}$ e $\left(\bar{x}, y_{2}\right) \in G_{i}^{\prime}$.

No caso (a), temos, da definição de $G_{i}$ e do fato de $y_{1} \neq y_{2}$, que o limite

$$
\lim _{\varepsilon \rightarrow 0} \hat{g}_{i}(\bar{x}, \varepsilon)=\frac{\partial \hat{f}}{\partial x_{i}}(\bar{x})
$$

não existe.

Pela hipótese de indução, $\hat{f} \uparrow\left(U \backslash C_{(1)}\right)$ é $\mathcal{C}^{1}$. Em particular, $\frac{\partial \hat{f}}{\partial x_{i}}(\bar{z})$ existe, para todo $\bar{z} \in U \backslash C_{(1)}$. Assim, devemos ter $\bar{x} \in C_{(1)}$.

No caso $(\mathrm{b})$, temos, de $\left(\bar{x}, y_{2}\right) \in G_{i}^{\prime}$, que $(\bar{x}, \pm \infty) \in G_{i}$. Então, novamente $\frac{\partial \hat{f}}{\partial x_{i}}(\bar{x})$ não existe. E, analogamente ao caso (a), $\bar{x} \in C_{(1)}$.

Portanto, $D_{i} \subseteq C_{(1)}$.

Como consequência desta inclusão e da hipótese de indução (i. e., $C_{(1)}$ tem interior vazio), temos $\stackrel{\circ}{D}_{i}=\emptyset$.

Uma vez que $\operatorname{Gr}\left(\hat{h}_{i}\right) \in C h(\mathcal{S})$, então $D_{i} \in C h(\mathcal{S})$. Logo, pelo Corolário 2.1.18, $D_{i}$ tem medida nula. Portanto, $\hat{h}_{i}$ é pseudofunção.

Vejamos, agora, que $\hat{h}_{i} \uparrow\left(U \backslash C_{(1)}\right)=\frac{\partial f^{*}}{\partial x_{i}}$, com $f^{*}=\hat{f} \uparrow\left(U \backslash C_{(1)}\right)$.

Com efeito, temos, pela hipótese de indução, que $f^{*}$ é $\mathcal{C}^{1}$. Então, pelo Lema 2.3.9, $G r\left(\frac{\partial f^{*}}{\partial x_{i}}\right)=G_{i}$.

É suficiente, portanto, mostrarmos que $\operatorname{Gr}\left(\hat{h}_{i} \uparrow\left(U \backslash C_{(1)}\right)\right)=G_{i}$.

Seja $(\bar{x}, y) \in \operatorname{Gr}\left(\hat{h}_{i} \uparrow\left(U \backslash C_{(1)}\right)\right)$. Então, $(\bar{x}, y) \in G_{i}$ ou $(\bar{x}, y) \in G_{i}^{\prime}$.

Se $(\bar{x}, y) \in G_{i}^{\prime}$, então $\bar{x} \in C_{(1)}$. Mas, do fato $(\bar{x}, y) \in G r\left(\hat{h}_{i} \uparrow\left(U \backslash C_{(1)}\right)\right)$, segue que $\bar{x} \in U \backslash C_{(1)}$. Contradição.

Portanto $(\bar{x}, y) \in G_{i}$. 
Por outro lado, se $(\bar{x}, y) \in G_{i}$, então, da definição de $\hat{h}_{i}$, concluímos que $(\bar{x}, y) \in G r\left(\hat{h}_{i}\right)$. Uma vez que

$$
G r\left(\frac{\partial f^{*}}{\partial x_{i}}\right)=G_{i}
$$

obtemos

$$
\bar{x} \in \operatorname{dom}\left(\frac{\partial f^{*}}{\partial x_{i}}\right)=U \backslash C_{(1)}
$$

Concluímos, assim, que $(\bar{x}, y) \in G r\left(\hat{h}_{i} \uparrow\left(U \backslash C_{(1)}\right)\right)$.

Agora, aplicando a hipótese de indução para $p-1, m=1$ a cada pseudofunção $\hat{h}_{i}$, teremos que existem fechados $C_{\hat{h}_{1}}, \ldots, C_{\hat{h}_{n}} \in C h(\mathcal{S})$, com interior vazio, tal que a restrição de $\hat{h}_{i}$ a $U \backslash C_{\hat{h}_{i}}$ é uma função $\mathcal{C}^{p-1}$, para cada $i=1, \ldots, n$.

Seja

$$
C=\bigcup_{i=1}^{n} C_{\hat{h}_{i}} \cup C_{(1)} .
$$

Então, $C$ é fechado e $C \in C h(\mathcal{S})$. Deste último e do Corolário 2.1.18, segue que $\stackrel{C}{C}=\emptyset$.

A fim de concluir a indução para o caso $m=1$, resta mostrar que $\hat{f} \uparrow(U \backslash C)$ é $\mathcal{C}^{p}$. Para tanto, basta verificarmos que $\frac{\partial f^{*}}{\partial x_{i}}, \operatorname{com} f^{*}=\hat{f} \uparrow(U \backslash C)$ e $i=1, \ldots, n$, é uma função $\mathcal{C}^{p-1}$.

De fato, seja $\bar{x} \in U \backslash C$. Então, $\bar{x} \in U \backslash C_{(1)}$ e $\bar{x} \in U \backslash C_{\hat{h}_{i}}$.

De $\bar{x} \in U \backslash C_{\hat{h}_{i}}$ e da hipótese de $\hat{h}_{i} \uparrow\left(U \backslash C_{\hat{h}_{i}}\right)$ ser $\mathcal{C}^{p-1}$, segue que $\hat{h}_{i}$ é $\mathcal{C}^{p-1}$ em $\bar{x}$.

Agora, em $U \backslash C_{(1)}$, as funções $\hat{h}_{i}$ e $\frac{\partial \hat{f}}{\partial x_{i}}$ assumem os mesmos valores. Logo, de $\bar{x} \in U \backslash C_{(1)}$, concluímos que $\frac{\partial \hat{f}}{\partial x_{i}}$ é $\mathcal{C}^{p-1}$.

Visto que este $\bar{x} \in U \backslash C$ é arbitrário, então $\frac{\partial \hat{f}}{\partial x_{i}}$ é $\mathcal{C}^{p-1}$ em $U \backslash C$.

Assim, a proposição é válida para $m=1$ e para todo $p \in \mathbb{N}$.

Agora, considere $m \geq 1$ e $p \in \mathbb{N}$ quaisquer.

Escreva $\hat{f}=\left(\hat{f}_{1}, \ldots, \hat{f}_{m}\right): U \rightarrow \mathbb{R}^{m}$. Então, $\hat{f}_{1}, \ldots, \hat{f}_{m}: U \rightarrow \mathbb{R}$ são pseudofunções.

Logo, existem fechados $C_{\hat{f}_{1}}, \ldots, C_{\hat{f}_{n}} \in C h(\mathcal{S})$, com interior vazio, de modo que a restrição 
de $\hat{f}_{j}$ a $U \backslash C_{\hat{f}_{j}}$ é uma função $\mathcal{C}^{p}$, para $j=1, \ldots, m$.

Seja

$$
C=\bigcup_{j=1}^{m} C_{\hat{f}_{j}}
$$

Então, $C$ é fechado, já que é união finita de fechados, e $C \in C h(\mathcal{S})$. Deste último e do Corolário 2.1.18, segue que $C$ tem interior vazio.

Fixemos $j \in\{1, \ldots, m\}$.

Visto que $\hat{f}_{j}$ restrita a $U \backslash C_{\hat{f}_{j}}$ é $\mathcal{C}^{p}$ e que $U \backslash C \subseteq U \backslash C_{\hat{f}_{j}}$, então $\hat{f}_{j} \uparrow(U \backslash C)$ é $\mathcal{C}^{p}$.

Portanto, $\hat{f}$ restrita a $U \backslash C$ é $\mathcal{C}^{p}$.

Com estes resultados preliminares firmados, podemos, enfim, assegurar o principal resultado desta terceira parte do Capítulo 2.

Teorema 2.3.14 (Seleção de uma função $\mathcal{C}^{p}$ ). Sejam $m, n \in \mathbb{N}^{+}$e $p \in \mathbb{N}$. Sejam, ainda, $A \in C h(\mathcal{S})_{n+m}$ e $B \subseteq \mathbb{R}^{n}$ um cubo tais que

$$
\forall \bar{x} \in B \exists \vec{y} \in \mathbb{R}^{m}(\bar{x}, \vec{y}) \in A
$$

Então, existem um cubo aberto $B^{\prime} \subseteq B$ e uma função $f: B^{\prime} \rightarrow \mathbb{R}^{m}$ que é $\mathcal{C}^{p}$ e, além disso, $G r(f) \in C h(\mathcal{S})_{n+m}$ e $\operatorname{Gr}(f) \subseteq A$.

Demonstração. Fixe $p \geq 1$.

Pelo Lema 2.3.1, temos que existem um cubo aberto $B^{*} \subseteq B$ e uma função contínua $f^{*}: B^{*} \rightarrow \mathbb{R}^{m}$ tal que $G r\left(f^{*}\right) \in C h(\mathcal{S})$ e $G r\left(f^{*}\right) \subseteq A$. Logo, pelo Teorema 2.3.13, existe um fechado $C \in C h(\mathcal{S})$, com interior vazio, tal que $f^{*} \uparrow\left(B^{*} \backslash C\right)$ é uma função $\mathcal{C}^{p}$.

Pelo Corolário 2.1.19, $B^{*} \backslash C$ tem ponto interior. Assim, $B^{*} \backslash C$ contém um cubo aberto $B^{\prime}$. Portanto, $f^{*} \uparrow B^{\prime}$ é $\mathcal{C}^{p}$.

Na próxima seção, usaremos este teorema apenas para o caso em que $p=1$. 


\subsection{Definindo a fronteira}

Observamos no enunciado do Teorema da Decomposição Celular uma suficiência para a validade dele, que é a Suposição 2.2.6. Um questionamento natural, então, seria em que circunstância a coleção $C h(\mathcal{S})$ logra tal propriedade. Nesta seção, provaremos que se os elementos de $C h(\mathcal{S})$ são projeções de conjuntos de zeros de funções $\mathcal{C}^{p}$, então suas fronteiras estão contidas em conjuntos em $C h(\mathcal{S})$ cujo interior é vazio.

Notação. Denotaremos por $\tilde{x}$ uma $k$-upla $\left(x_{1}, \ldots, x_{k}\right)$ e por $\hat{y}$ uma l-upla $\left(y_{1}, \ldots, y_{l}\right)$.

Lema 2.4.1. Sejam $k, l \in \mathbb{N}^{+}$e $g: \mathbb{R}^{k+l} \rightarrow[0, \infty)$ uma função $\mathcal{C}^{1}$. Considere os conjuntos,

$$
F_{g}=\left\{(\tilde{x}, \varepsilon): \varepsilon>0 \wedge \exists \hat{y}\left(g(\tilde{x}, \hat{y})=\varepsilon \wedge \bigwedge_{i=1}^{l} \frac{\partial g}{\partial y_{i}}(\tilde{x}, \hat{y})=0\right)\right\}
$$

$e$

$$
E_{g}=\left\{\tilde{x}:(\tilde{x}, 0) \in \bar{F}_{g}\right\}
$$

Seja ainda $\tilde{a} \in \mathbb{R}^{k}$ tal que $\tilde{a} \in \delta\left(\pi_{k+l, k}[Z(g)]\right)^{18}$, com $Z(g)$ o conjunto dos zeros de $g$. Suponha, além disso, que $\{\hat{y}: g(\tilde{a}, \hat{y})=0\}$ tenha um número finito (não nulo) de componentes conexas e que, pelo menos, uma destas é limitada.

Então, $\tilde{a} \in E_{g}$.

Demonstração. Seja $P \subseteq \mathbb{R}^{l}$ uma componente conexa limitada de $Y=\{\hat{y}: g(\tilde{a}, \hat{y})=0\}$.

Seja $H \subseteq \mathbb{R}^{l}$ fechado e limitado, de sorte que $P \subseteq \stackrel{\circ}{H}$ e a intersecção de $H$ com qualquer outra componente conexa de $Y$ é vazia.

Vejamos que tal $H$ existe.

Com efeito, como $g(\tilde{a}, \hat{y})=g_{\tilde{a}}(\hat{y})$, para $\tilde{a}$ fixado, é contínua e $\{0\} \subseteq \mathbb{R}$ é fechado de $\mathbb{R}$, então $\{\hat{y}: g(\tilde{a}, \hat{y})=0\}=g_{\tilde{a}}^{-1}[\{0\}]$ é fechado de $\mathbb{R}^{l}$. Logo, as componentes conexas de $Y$ são fechados de $\mathbb{R}^{l}$.

\footnotetext{
${ }^{18}$ Lembre-se de que $\delta\left(\pi_{k+l, k}[Z(g)]\right)$ denota a fronteira de $\pi_{k+l, k}[Z(g)]$.
} 
Sejam $C_{1}, \ldots, C_{t}$ as componentes conexas de $Y$ e assuma, sem perda de generalidade, que $C_{1}=P$. Então, pela definição de componente conexa,

$$
P \subseteq \mathbb{R}^{l} \backslash \bigcup_{j \neq 1} C_{j}
$$

Logo, para cada $\hat{y} \in P$, temos $\hat{y} \in \mathbb{R}^{l} \backslash \bigcup_{j \neq 1} C_{j}$. Uma vez que $\mathbb{R}^{l} \backslash \bigcup_{j \neq 1} C_{j}$ é aberto, temos que existe um cubo aberto $B_{\hat{y}}$, que contém $\hat{y}$, tal que $B_{\hat{y}} \subseteq \mathbb{R}^{l} \backslash \bigcup_{j \neq 1} C_{j}$. Assim,

$$
P \subseteq \bigcup_{\hat{y} \in P} B_{\hat{y}} \subseteq \mathbb{R}^{l} \backslash \bigcup_{j \neq 1} C_{j}
$$

Ou ainda,

$$
P \subseteq \bigcup_{\hat{y} \in P} B_{\hat{y}}^{\prime} \subseteq \bigcup_{\hat{y} \in P} \overline{B_{\hat{y}}^{\prime}} \subseteq \bigcup_{\hat{y} \in P} B_{\hat{y}} \subseteq \mathbb{R}^{l} \backslash \bigcup_{j \neq 1} C_{j},
$$

com $B_{\hat{y}}^{\prime}$ 's cubos abertos.

Como $P$ é um compacto de $\mathbb{R}^{l}$, já que é fechado e limitado, então existem finitos cubos abertos $B_{1}^{\prime}, \ldots, B_{r}^{\prime} \in\left\{B_{\hat{y}}^{\prime}: \hat{y} \in P\right\}$ tais que

$$
P \subseteq B_{1}^{\prime} \cup \cdots \cup B_{r}^{\prime}
$$

Tome

$$
H=\overline{\bigcup_{j=1}^{r} B_{j}^{\prime}}=\bigcup_{j=1}^{r} \overline{B_{j}^{\prime}}
$$

Então,

$$
P \subseteq \bigcup_{j=1}^{r} B_{j}^{\prime} \subseteq H \subseteq \mathbb{R}^{l} \backslash \bigcup_{j \neq 1} C_{j}
$$


De $P \subseteq \bigcup_{j=1}^{r} B_{j}^{\prime} \subseteq H$, temos que

$$
P \subseteq \bigcup_{j=1}^{r} B_{j}^{\prime}=\bigcup_{j=1}^{r} \dot{\circ}_{j}^{\prime} \subseteq \widehat{\bigcup_{j=1}^{r} B_{j}^{\prime}} \subseteq \stackrel{\circ}{H} .
$$

De $H \subseteq \mathbb{R}^{l} \backslash \bigcup_{j \neq 1} C_{j}$, temos que

$$
H \cap C_{j}=\emptyset, \text { para } j \in\{2, \ldots, t\} .
$$

Assim, segue a existência de $H$.

Agora, por hipótese, $\tilde{a} \in \delta\left(\pi_{k+l, k}[Z(g)]\right)$. Então, $\tilde{a} \in \overline{\mathbb{R}^{k} \backslash \pi_{k+l, k}[Z(g)]}$. Consequentemente, existe uma sequência $\left(\tilde{a}_{j}\right)_{j}$ em $\mathbb{R}^{k} \backslash \pi_{k+l, k}[Z(g)]$ tal que $\tilde{a}_{j} \rightarrow \tilde{a}$. Logo,

$$
\text { para todo } j \in \mathbb{N} \quad \text { e todo } \hat{y} \in \mathbb{R}^{l}, \quad g\left(\hat{a}_{j}, \hat{y}\right)>0
$$

ou seja

$$
\text { (*) } \left.\quad \text { para qualquer } j \in \mathbb{N}, \quad g\left[\left\{\tilde{a}_{j}\right\} \times \mathbb{R}^{l}\right] \subseteq\right] 0, \infty[\text {. }
$$

Como $H$ é compacto e $g_{\tilde{a}_{j}}\left(i\right.$. e., $g_{\tilde{a}_{j}}(\hat{y})=g\left(\tilde{a}_{j}, \hat{y}\right)$, para $\tilde{a}_{j}$ fixado e $\hat{y} \in \mathbb{R}^{l}$ arbitrário) é contínua, para todo $j \in \mathbb{N}$, então pelo Teorema de Weierstrass, $g_{\tilde{a}_{j}}$ atinge máximo e mínimo em $H$.

Para cada $j \in \mathbb{N}$, seja $\delta_{j} \in \mathbb{R}$ dado por

$$
\delta_{j}=\min \left\{g\left(\tilde{a}_{j}, \hat{y}\right): \hat{y} \in H\right\}
$$

De $\left(^{*}\right)$, segue que $\delta_{j}>0$, para todo $j \in \mathbb{N}$.

Como $H \cap Y \neq \emptyset$, então existe $\hat{p} \in H$ tal que $g(\tilde{a}, \hat{p})=0$. Uma vez que $\left(\tilde{a}_{j}, \hat{p}\right) \rightarrow(\tilde{a}, \hat{p})$ e $g$ 
é contínua, temos

$$
g\left(\tilde{a}_{j}, \hat{p}\right) \rightarrow g(\tilde{a}, \hat{p})=0
$$

Logo, $\delta_{j} \rightarrow 0$

O objetivo, agora, é mostrar que para $j$ arbitrariamente grande, temos $\left(\tilde{a}_{j}, \delta_{j}\right) \in F_{g}$. Pois, desta forma, $(\tilde{a}, 0) \in \bar{F}_{g}$ e, portanto, $\tilde{a} \in E_{g}$.

Seja $\hat{b}_{j} \in H$ tal que $g\left(\tilde{a}_{j}, \hat{b}_{j}\right)=\delta_{j}$, para cada $j \in \mathbb{N}$.

Como $H$ é limitado, então existe $\left(\hat{b}_{j_{s}}\right)_{s}$ subsequência convergente. Digamos, $\hat{b}_{j_{s}} \rightarrow \hat{b}$.

Também, $H$ é fechado, logo $\hat{b} \in H$.

Uma vez que $\left(\tilde{a}_{j}, \hat{b}_{j_{s}}\right) \rightarrow(\tilde{a}, \hat{b})$ e $g$ é contínua, obtemos

$$
\delta_{j_{s}}=g\left(\tilde{a}_{j}, \hat{b}_{j_{s}}\right) \rightarrow g(\tilde{a}, \hat{b})
$$

Mas, $\lim _{s \rightarrow \infty} \delta_{j_{s}}=0$. Portanto, $g(\tilde{a}, \hat{b})=0$.

Assim,

$$
\hat{b} \in Y \cap H=\left(P \cup C_{2} \cup \cdots \cup C_{t}\right) \cap H .
$$

Como $H \cap C_{j}=\emptyset$, para todo $j \in\{2, \ldots, t\}$, então $\hat{b} \in P$. Também, como $P \subseteq \stackrel{\circ}{H}, \operatorname{logo}$ $\hat{b} \in \stackrel{\circ}{H}$. Assim, existe um cubo aberto $B$ de centro $\hat{b}$ tal que $B \subseteq H$. Uma vez que $\hat{b}_{j_{s}} \rightarrow \hat{b}$, temos, pela definição de convergência, que existe $J \in \mathbb{N}$ tal que, para todo $s>J, \hat{b}_{j_{s}} \in B \subseteq H$ e, assim, $\hat{b}_{j_{s}} \in \stackrel{\circ}{H}$.

Vejamos que $\frac{\partial g}{\partial y_{i}}\left(\tilde{a}_{j_{s}}, \hat{b}_{j_{s}}\right)=0$, para $i=1, \ldots, l$ e $s>J$.

Com efeito, fixemos $s>J$ e $i \in\{1, \ldots, l\}$.

Seja $g_{\tilde{a}_{j_{s}}}: \stackrel{\circ}{H} \rightarrow(0, \infty)$ dada por

$$
g_{\tilde{a}_{j_{s}}}(\hat{y})=g\left(\tilde{a}_{j_{s}}, \hat{y}\right)
$$

Então, $g_{\tilde{a}_{j_{s}}}$ é diferenciável, já que $g$ o é. 
De

$$
\delta_{j_{s}}=g\left(\tilde{a}_{j_{s}}, \hat{b}_{j_{s}}\right)=\min \left\{g\left(\tilde{a}_{j_{s}}, \hat{y}\right): \hat{y} \in H\right\} \leq \min \left\{g\left(\tilde{a}_{j_{s}}, \hat{y}\right): \hat{y} \in \stackrel{\circ}{H}\right\} \text { e } \hat{b}_{j_{s}} \in \stackrel{\circ}{H}
$$

temos que a função $g_{\tilde{a}_{j_{s}}}$ assume valor mínimo em $\hat{b}_{j_{s}} \in \stackrel{\circ}{H}$. Portanto,

$$
\frac{\partial g_{\tilde{a}_{j_{s}}}}{\partial y_{i}}\left(\hat{b}_{j_{s}}\right)=0
$$

ou seja,

$$
\frac{\partial g}{\partial y_{i}}\left(\tilde{a}_{j_{s}}, \hat{b}_{j_{s}}\right)=0
$$

Assim, para todo $s>J$,

$$
g\left(\tilde{a}_{j_{s}}, \hat{b}_{j_{s}}\right)=\delta_{j_{s}}>0 \text { e } \frac{\partial g}{\partial y_{i}}\left(\tilde{a}_{j_{s}}, \hat{b}_{j_{s}}\right)=0
$$

para $i=1, \ldots, l$.

Então, pela definição de $F_{g},\left(\tilde{a}_{j_{s}}, \delta_{j_{s}}\right)_{s>J} \in F_{g}$ e, uma vez que $\left(\tilde{a}_{j_{s}}, \delta_{j_{s}}\right) \rightarrow(\tilde{a}, 0)$, temos $(\tilde{a}, 0) \in \bar{F}_{g}$. Ou seja, $\tilde{a} \in E_{g}$.

Aqui, vale notar que este Lema 2.4.1 possui um caráter geral, no sentido em que não se exige que os conjuntos $G r(g), F_{g}$ e $E_{g}$ estejam em $C h(\mathcal{S})$.

Teorema 2.4.2 (Teorema do Complemento $^{19}$ ). Sejam $n, m \in \mathbb{N}^{+}$. Seja, ainda, $A \in C h(\mathcal{S})_{n}$ tal que podemos escrever

$$
A=\{\bar{x}: \exists \vec{y} f(\bar{x}, \vec{y})=0\}
$$

sendo que $f: \mathbb{R}^{n+m} \rightarrow \mathbb{R}$ é $\mathcal{C}^{p}$ e $G r(f) \in C h(\mathcal{S})_{n+m+1}$.

Então, existe $T \in C h(\mathcal{S})_{n}$ tal que $T$ tem interior vazio e $\delta(A) \subseteq T$.

\footnotetext{
${ }^{19}$ Este teorema é assim intitulado, pois sua validade é suficiente para a validade do Teorema da Decomposição Celular para $C h(\mathcal{S})$ (Teorema 2.2.7), que, por sua vez, tem como consequência o fato de $C h(\mathcal{S})$ ser fechado sob a operação do complemento.
} 
Demonstração. Vamos começar apresentando um conjunto $T \subseteq \mathbb{R}^{n}$, que verificamos estar em $C h(\mathcal{S})$, e, então, provamos as afirmações: $T$ tem interior vazio e $\delta(A) \subseteq T$.

Primeiramente, seja $g: \mathbb{R}^{n+1+2 m} \rightarrow \mathbb{R}$ definida por

$$
g(\bar{x}, q, \vec{y}, \vec{z})=(f(\bar{x}, \vec{y}))^{2}+\sum_{i=1}^{m}\left(q^{2}-\left(y_{i}^{2}+z_{i}^{2}\right)\right)^{2} .
$$

AfirmaÇÃo 1. $\operatorname{Gr}(g) \in C h(\mathcal{S})_{n+1+2 m+1}$.

Prova da Afirmação. Seja $\phi(\bar{x}, q, \vec{y}, \vec{z}, v)$ dada por

$$
\exists u\left(\phi_{G r(f)}(\bar{x}, \vec{y}, u) \wedge v=u^{2}+\sum_{i=1}^{m}\left(q^{2}-\left(y_{i}^{2}+z_{i}^{2}\right)\right)^{2}\right)
$$

em que $\langle\overline{\mathbb{R}},\{Y: Y \in C h(\mathcal{S})\}\rangle \models \phi_{G r(f)}(\bar{x}, \vec{y}, u)$ se, e somente se, $(\bar{x}, \vec{y}, u) \in G r(f)$.

Assim, $\phi(\bar{x}, q, \vec{y}, \vec{z}, v)$ é uma $C h$-fórmula e

$$
G r(g)=\{(\bar{x}, q, \vec{y}, \vec{z}, v):\langle\overline{\mathbb{R}},\{Y: Y \in C h(\mathcal{S})\}\rangle=\phi(\bar{x}, q, \vec{y}, \vec{z}, v)\}
$$

Portanto, $\operatorname{Gr}(g) \in C h(\mathcal{S})$.

AFIRMAÇÃo 2. $\operatorname{Im}(g) \subseteq[0, \infty[$.

Prova da Afirmação. Para todos $\bar{x} \in \mathbb{R}^{n}, \vec{y}, \vec{z} \in \mathbb{R}^{m}$ e $q \in \mathbb{R}$, temos

$$
(f(\bar{x}, \vec{y}))^{2}, \sum_{i=1}^{m}\left(q^{2}-\left(y_{i}^{2}+z_{i}^{2}\right)\right)^{2} \geq 0
$$

Logo, $g(\bar{x}, q, \vec{y}, \vec{z}) \geq 0$.

AfirmaÇÃO 3. $\operatorname{Gr}\left(\frac{\partial g}{\partial x_{i}}\right) \in C h(\mathcal{S})$, para $i=1, \ldots, n+1+2 m$.

Prova da Afirmação. Uma vez que $g$ é uma função $\mathcal{C}^{1}$, então, pelo Lema 2.3.9, segue que $\operatorname{Gr}\left(\frac{\partial g}{\partial x_{i}}\right) \in C h(\mathcal{S})$, para todo $i=1, \ldots, n+1+2 m$. 
AFIRMAÇ̃̃̃ 4 . Existe $\vec{z}$ tal que $g(\bar{x}, q, \vec{y}, \vec{z})=0$ se, e somente se, $f(\bar{x}, \vec{y})=0$ e $\left|y_{i}\right| \leq|q|$, para $i=1, \ldots, m$.

Prova da Afirmação.

$(\Rightarrow)$ Por hipótese, existe $\vec{z} \in \mathbb{R}^{m}$ tal que

$$
g(\bar{x}, q, \vec{y}, \vec{z})=0=(f(\bar{x}, \vec{y}))^{2}+\sum_{i=1}^{m}\left(q^{2}-\left(y_{i}^{2}+z_{i}^{2}\right)\right)^{2}
$$

Então, $f(\bar{x}, \vec{y})=0$ e $q^{2}-\left(y_{i}^{2}+z_{i}^{2}\right)=0$, para $i=1, \ldots, m$.

Mas, $q^{2}-\left(y_{i}^{2}+z_{i}^{2}\right)=0$ é equivalente a $q^{2}-y_{i}^{2}=z_{i}^{2}$ que, por sua vez, é equivalente a $|q|^{2}-\left|y_{i}\right|^{2}=z_{i}^{2} \geq 0$.

$(\Leftarrow)$ Por hipótese, $f(\bar{x}, \vec{y})=0$ e $\left|y_{i}\right| \leq|q|$, para $i=1, \ldots, m$.

Tome $z_{i}=\sqrt{|q|^{2}-\left|y_{i}\right|^{2}}$. Então, $q^{2}-\left(y_{i}^{2}+z_{i}^{2}\right)=|q|^{2}-\left|y_{i}\right|^{2}-z_{i}^{2}=0$. Logo,

$$
\sum_{i=1}^{m}\left(q^{2}-\left(y_{i}^{2}+z_{i}^{2}\right)\right)^{2}=0
$$

Portanto, $g(\bar{x}, q, \vec{y}, \vec{z})=0$.

AfirmaÇÃo 5. Seja $(\bar{a}, c) \in \mathbb{R}^{n} \times[0,+\infty[$. Então,

$$
Z^{\prime}=\{(\vec{y}, \vec{z}): g(\bar{a}, c, \vec{y}, \vec{z})=0\} \subseteq[-c, c]^{2 m}
$$

Prova da Afirmação. Seja $(\vec{y}, \vec{z}) \in Z^{\prime}$. Então, $g(\bar{a}, c, \vec{y}, \vec{z})=0$. Pela Afirmação 4,

$$
\left|y_{i}\right| \leq|c|=c, i=1, \ldots, m
$$

Além disso, $g(\bar{a}, c, \vec{y}, \vec{z})=0 \Rightarrow z_{i}^{2}=c^{2}-y_{i}^{2}$, para $i=1, \ldots, m$. Logo,

$$
\left|z_{i}\right|=\sqrt{z_{i}^{2}}=\sqrt{c^{2}-y_{i}^{2}} \leq \sqrt{c^{2}}=|c|=c .
$$


Assim, $(\vec{y}, \vec{z}) \in[-c, c]^{m} \times[-c, c]^{m}=[-c, c]^{2 m}$.

Agora, analogamente ao Lema 2.4.1, definimos:

$$
\begin{gathered}
F=\left\{(\bar{x}, q, \varepsilon): \varepsilon>0 \wedge \exists \vec{y} \exists \vec{z}\left[g(\bar{x}, q, \vec{y}, \vec{z})=\varepsilon \wedge \bigwedge_{i=1}^{m}\left(\frac{\partial g}{\partial y_{i}}(\bar{x}, q, \vec{y}, \vec{z})=0\right) \wedge \bigwedge_{i=1}^{m}\left(\frac{\partial g}{\partial z_{i}}(\bar{x}, q, \vec{y}, \vec{z})=0\right)\right]\right\}, \\
E=\{(\bar{x}, q):(\bar{x}, q, 0) \in \bar{F}\}, \\
Q=\left\{(\bar{x}, r): \exists q\left((\bar{x}, q) \in E \wedge q>0 \wedge q=\frac{1}{r}\right)\right\}
\end{gathered}
$$

e

$$
T=\{\bar{x}:(\bar{x}, 0) \in \bar{Q}\}
$$

Pelas Afirmações 1 e $3, G r(g) \in C h(\mathcal{S})$ e $\operatorname{Gr}\left(\frac{\partial g}{\partial y_{i}}\right), \operatorname{Gr}\left(\frac{\partial g}{\partial z_{i}}\right) \in C h(\mathcal{S}), i=1, \ldots, m$. Assim, $F \in C h(\mathcal{S})_{n+2}$.

Também, seja $\phi_{F}(\bar{x}, q, 0)$ interpretada em $\langle\overline{\mathbb{R}},\{Y: Y \in C h(\mathcal{S})\}\rangle$ como $(\bar{x}, q, 0) \in F$. Então,

$$
E=\left\{(\bar{x}, q):\langle\overline{\mathbb{R}},\{Y: Y \in C h(\mathcal{S})\}\rangle \models \overline{\phi_{F}(\bar{x}, q, 0)}\right\} .
$$

Logo, $E \in C h(\mathcal{S})_{n+1}$.

Analogamente,

$$
Q=\left\{(\bar{x}, r):\langle\overline{\mathbb{R}},\{Y: Y \in C h(\mathcal{S})\}\rangle \models \exists q\left(\phi_{E}(\bar{x}, q) \wedge q>0 \wedge q r=1\right)\right\},
$$

$\operatorname{com} \phi_{E}(\bar{x}, q)$ interpretada como $(\bar{x}, q) \in E$.

Portanto, $Q \in C h(\mathcal{S})_{n+1}$.

E, finalmente

$$
T=\left\{\bar{x}:\langle\overline{\mathbb{R}},\{Y: Y \in C h(\mathcal{S})\}\rangle \models \overline{\phi_{Q}(\bar{x}, 0)}\right\},
$$

de modo que $\phi_{Q}(\bar{x}, 0)$ é interpretada como $(\bar{x}, 0) \in Q$.

Assim, $T \in C h(\mathcal{S})_{n}$. 
AFIRMAÇÃO 6. T tem interior vazio.

Prova da Afirmação. Suponha, por absurdo, que $\stackrel{\circ}{T} \neq \emptyset$. Então, pelo Lema 2.3.2, $Q$ tem interior não vazio.

Então, $E$ tem interior não vazio.

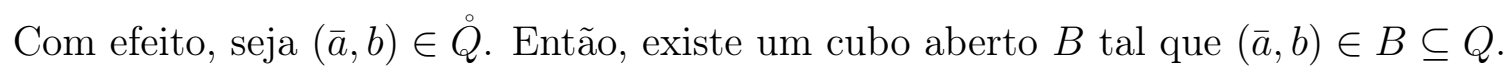

Escrevamos $B=] p_{1}, q_{1}[\times \cdots \times] p_{n}, q_{n}[\times] p_{n+1}, q_{n+1}\left[\right.$. Assim, $p_{n+1}<b<q_{n+1}$.

Seja

$$
\mu=\frac{1}{2} \min \left\{q_{n+1}-b, b-p_{n+1}\right\}
$$

Então, $] b-\mu, b+\mu[\subseteq] p_{n+1}, q_{n+1}[$.

Considere

$$
\left.B^{\prime}=\right] p_{1}, q_{1}[\times \cdots \times] p_{n}, q_{n}[\times] \frac{1}{b+\mu}, \frac{1}{b-\mu}[
$$

Então, $B^{\prime} \subseteq E$.

De fato, se $(\bar{x}, t) \in B^{\prime}$, então

$$
\left.\bar{x} \in \prod_{i=1}^{n}\right] p_{i}, q_{i}\left[\text { e } \frac{1}{b+\mu}<t<\frac{1}{b-\mu}\right.
$$
Q

Deste último, temos $\left.\frac{1}{t} \in\right] b-\mu, b+\mu\left[\right.$. E, assim, $\left(\bar{x}, \frac{1}{t}\right) \in B \subseteq Q$. Logo, pela definição de

$$
(\bar{x}, t)=\left(\bar{x}, \frac{1}{1 / t}\right) \in E
$$

Portanto, $\stackrel{\circ}{E} \neq \emptyset$.

Consequentemente, pelo Lema 2.3.2, $F$ tem um ponto interior. Então, existem um ponto $(\bar{a}, q) \in \mathbb{R}^{n+1}$ e um intervalo real aberto $I^{\prime}$ tal que $\{(\bar{a}, q)\} \times I^{\prime} \subseteq F$. 
Seja a função $g^{*}: \mathbb{R}^{2 m} \rightarrow \mathbb{R}$ dada por

$$
g^{*}(\vec{y}, \vec{z})=g(\bar{a}, q, \vec{y}, \vec{z})
$$

Definamos

$$
G_{g^{*}}=\left\{(t, \vec{y}, \vec{z}): g^{*}(\vec{y}, \vec{z})=t\right\}
$$

Uma vez que $\operatorname{Gr}\left(g^{*}\right) \in C h(\mathcal{S})$, segue que $G_{g^{*}} \in C h(\mathcal{S})$.

De $\{(\bar{a}, q)\} \times I^{\prime} \subseteq F$ e da definição de $F$, temos que

$$
\text { para todo } t \in I^{\prime} \text { existe }(\vec{y}, \vec{z}) \in \mathbb{R}^{2 m} \text { de sorte que } g^{*}(\vec{y}, \vec{z})=t
$$

i. e.

$$
(t, \vec{y}, \vec{z}) \in G_{g^{*}}
$$

Logo, pelo Teorema 2.3.14, existem um intervalo aberto $I \subseteq I^{\prime}$ e uma função $C^{1} \gamma: I \rightarrow$ $\mathbb{R}^{2 m}$ tais que $\operatorname{Gr}(\gamma) \in C h(\mathcal{S})$ e $G r(\gamma) \subseteq G_{g^{*}}$.

Então, $g^{*} \circ \gamma: I \rightarrow I$ é a identidade.

De fato, dado $t \in I$, temos que $(t, \gamma(t)) \in G_{g^{*}}$. Pela definição de $G_{g^{*}}, g^{*}(\gamma(t))=t$.

Agora, pela Regra da Cadeia, $d\left(g^{*} \circ \gamma\right)(t)=d g^{*}(\gamma(t)) \circ d \gamma(t)$. Mas, pela definição de $F$, temos

$$
\frac{\partial g^{*}}{\partial y_{i}}(\gamma(t))=0=\frac{\partial g^{*}}{\partial z_{i}}(\gamma(t)), i=1, \ldots, m
$$

Logo, $d g^{*}(\gamma(t))=0$. Portanto, $d\left(g^{*} \circ \gamma\right)(t)=0$.

Assim, $g^{*} \circ \gamma=$ cte. $(\Rightarrow \Leftarrow)$

AfirmaÇÃO $7 . \delta(A) \subseteq T$.

Prova da Afirmação. Primeiro, introduzimos uma notação que será usada ao longo da demonstração. 
Notação. Se $\vec{y} \in \mathbb{R}^{m}$, então $\|\vec{y}\|=\max \left\{\left|y_{i}\right|: i=1, \ldots, m\right\}$.

Seja $\bar{a} \in \delta(A)$. Temos, então, dois casos a considerar: $\bar{a} \in A$ e $\bar{a} \notin A$.

(1) $\bar{a} \in A$.

Então, pela definição de $A$, existe $\vec{b} \in \mathbb{R}^{m}$ tal que $f(\bar{a}, \vec{b})=0$.

Seja $\|\vec{b}\|=q_{0}$. Seja $q \in\left[q_{0}, \infty\left[\right.\right.$. Logo, pela Afirmação 4 , existe $\vec{z} \in \mathbb{R}^{m}$ de sorte que $g(\bar{a}, q, \vec{b}, \vec{z})=0$. Portanto,

$$
(\dagger) \quad(\bar{a}, q) \in \pi_{n+1+2 m, n+1}[Z(g)] \text {, para todo } q \in\left[q_{0}, \infty[\text {. }\right.
$$

Uma vez que $\bar{a} \in \delta(A)$, então existe uma sequência $\left(\bar{a}_{j}\right)_{j}$ em $\mathbb{R}^{n} \backslash A$ tal que $\bar{a}_{j} \rightarrow \bar{a}$. Logo, para todo $j \in \mathbb{N}$ e todo $\vec{y} \in \mathbb{R}^{m}$, temos $f\left(\bar{a}_{j}, \vec{y}\right) \neq 0$. Consequentemente, pela Afirmação 4 ,

$$
g\left(\bar{a}_{j}, q, \vec{y}, \vec{z}\right) \neq 0 \text {, para qualquer }(q, \vec{y}, \vec{z}) \in \mathbb{R}^{1+2 m} \text { e qualquer } j \in \mathbb{N} \text {. }
$$

Portanto, $\left(\bar{a}_{j}, q\right) \notin \pi_{n+1+2 m, n+1}[Z(g)]$, para $j \in \mathbb{N}$ e $q \in \mathbb{R}$ arbitrários.

Desta última afirmação e de $(\dagger)$, segue que

$$
\text { qualquer que seja } q \in\left[q_{0}, \infty\left[, \quad(\bar{a}, q) \in \delta\left(\pi_{n+1+2 m, n+1}[Z(g)]\right)\right. \text {. }\right.
$$

Seja $Z^{\prime \prime}=\{(\vec{y}, \vec{z}): g(\bar{a}, q, \vec{y}, \vec{z})=0\}$.

Então, $Z^{\prime \prime} \in C h(\mathcal{S})_{2 m}$.

Como $\mathbb{R}^{2 m}$ é afim, então, por $(E F 5)$ para $Z^{\prime \prime}, Z^{\prime \prime}\left(=Z^{\prime \prime} \cap \mathbb{R}^{2 m}\right)$ tem finitas componentes conexas. Pela Afirmação 5, concluímos que elas são limitadas. Além disso, como $g$ é $\mathcal{C}^{1}$, então, pelo Lema 2.4.1 para $k=n+1$ e $l=2 m$, obtemos

$$
(\bar{a}, q) \in E,
$$

para todo $q \in\left[q_{0}, \infty[\right.$. 
Assim,

se $q_{0} \neq 0$, então $(\bar{a}, r) \in Q$, para todo $\left.r \in\right] 0, \frac{1}{q_{0}}[$;

se $q_{0}=0$, então $(\bar{a}, r) \in Q$, para todo $\left.r \in\right] 0, \infty[$.

Em ambos os casos, $(\bar{a}, 0) \in \bar{Q}$. Portanto, $\bar{a} \in T$.

(2) $\bar{a} \notin A$.

Como $\bar{a} \in \delta(A)$, então existe uma sequência $\left(\bar{a}_{j}\right)_{j}$ em $A$ tal que $\bar{a}_{j} \rightarrow \bar{a}$.

Logo, para todo $j \in \mathbb{N}$ existe $\vec{d}_{j}$ tal que $f\left(\bar{a}_{j}, \vec{d}_{j}\right)=0$.

Vejamos que existe $\left(\vec{b}_{j}\right)_{j}$, de modo que $\left\|\vec{b}_{j}\right\|=\min \left\{\|\vec{y}\|: f\left(\bar{a}_{j}, \vec{y}\right)=0\right\}$.

De fato, fixe $j \in \mathbb{N}$.

Seja $Z_{j}=\left\{\vec{y}: f\left(\bar{a}_{j}, \vec{y}\right)=0\right\}$. Então, $Z_{j} \neq \emptyset$, pois $\vec{d}_{j} \in Z_{j}$. Também, $Z_{j}$ é fechado.

Considere $\mu=\left\|\overrightarrow{d_{j}}\right\|+1 \mathrm{e}$

$$
\bar{B}=[-\mu, \mu] \times \cdots \times[-\mu, \mu] \subseteq \mathbb{R}^{m}
$$

Então,

$$
\overrightarrow{d_{j}}=\left(d_{1_{j}}, \ldots, d_{m_{j}}\right) \in \bar{B}
$$

pois $\left|d_{i_{j}}\right| \leq\left\|\vec{d}_{j}\right\|<\mu$, para $i=1, \ldots, m$.

Assim, $B^{\prime}=Z_{j} \cap \bar{B} \neq \emptyset$. Além disso, $B^{\prime}$ é compacto (pois, é fechado e limitado).

Considere a função $h: \mathbb{R}^{m} \rightarrow \mathbb{R}$ dada por

$$
h(\vec{y})=\|\vec{y}\|
$$

Então, $h$ é contínua. Logo, pelo Teorema de Weierstrass, $B^{\prime}$ tem ponto de mínimo local, ou seja,

existe $\vec{b}_{j} \in B^{\prime}$ tal que para todo $\vec{y} \in B^{\prime}, \quad h\left(\vec{b}_{j}\right) \leq h(\vec{y})$. 
Vejamos, agora, que para todo $\vec{y} \in Z_{j}, h\left(\vec{b}_{j}\right) \leq h(\vec{y})\left(i\right.$. e., $\vec{b}_{j}$ é ponto de mínimo local em $\left.Z_{j}\right)$

Com efeito, seja $\vec{y} \in Z_{j} \backslash B^{\prime}$ arbitrário. Então, existe $i \in\{1, \ldots, m\}$ tal que $\left|y_{i}\right|>\mu$.

Mas, $h(\vec{y})=\|\vec{y}\| \geq\left|y_{i}\right|$ e $\mu>\left\|\vec{d}_{j}\right\| \geq h\left(\vec{b}_{j}\right)$, já que $\vec{d}_{j} \in B^{\prime}$. Assim, $h(\vec{y})>h\left(\vec{b}_{j}\right)$.

Portanto, $\left\|\vec{b}_{j}\right\|=\min \left\{\|\vec{y}\|: \vec{y} \in Z_{j}\right\}=\min \{\|\vec{y}\|: f(\bar{a}, \vec{y})=0\}$, ou seja, $\vec{b}_{j}$ é ponto de mínimo local em $Z_{j}$.

Agora, chamemos $\left\|\vec{b}_{j}\right\|$ de $q_{j}$, para $j \in \mathbb{N}$.

Suponha, por absurdo, que $\left(q_{j}\right)_{j}$ é limitada. Então, $\left(\vec{b}_{j}\right)_{j}$ também o é.

Com efeito, se $\left(q_{j}\right)_{j}$ é limitada, então, por definição, existem $p, q \in \mathbb{R}$ tal que $\left.\left\|\vec{b}_{j}\right\| \in\right] p, q[$, para todo $j$.

Como $\left\|\vec{b}_{j}\right\|=\max \left\{\left|b_{1_{j}}\right|, \ldots,\left|b_{m_{j}}\right|\right\}, \operatorname{com} \vec{b}_{j}=\left(b_{1_{j}}, \ldots, b_{m_{j}}\right)$, então

$$
\left|b_{i_{j}}\right| \leq\left\|\vec{b}_{j}\right\|<q, \text { para } i \in\{1, \ldots, m\}
$$

i. e.,

$$
-q<b_{i_{j}}<q \text {, para todo } j \in \mathbb{N} \text { e todo } i \in\{1, \ldots, m\}
$$

Logo, tomando $B=]-q, q[\times \cdots \times]-q, q\left[\subseteq \mathbb{R}^{m}\right.$, obtemos $\vec{b}_{j} \in B$, para todo $j$.

Assim, $\left(\vec{b}_{j}\right)_{j}$ tem subsequência $\left(\vec{b}_{j_{s}}\right)_{s}$ convergente. Digamos, $\vec{b}_{j_{s}} \rightarrow \vec{b}$.

Visto que, $f\left(\bar{a}_{j_{s}}, \vec{b}_{j_{s}}\right) \rightarrow f(\bar{a}, \vec{b})$ e $f\left(\bar{a}_{j_{s}}, \vec{b}_{j_{s}}\right)=0$, para todo $s \in \mathbb{N}$, concluímos que

$$
f(\bar{a}, \vec{b})=0
$$

Portanto, $\bar{a} \in A .(\Rightarrow \Leftarrow)$

Consequentemente, $\left(q_{j}\right)_{j}$ é ilimitada.

Logo, para todo $r>0$, existe $q_{j_{s}} \in\left\{q_{j}: j \in \mathbb{N}\right\}$ tal que $\left.q_{j_{s}} \notin\right]-r, r[$.

Uma vez que $q_{j} \geq 0$, para todo $j \in \mathbb{N}$, obtemos $q_{j_{s}}>0$. Em outros termos, $\left(q_{j}\right)_{j}$ possui 
subsequência cujos termos são todos positivos.

Assim, podemos considerar, sem perda de generalidade, que $q_{j}>0$, para qualquer $j \in \mathbb{N}$. Agora, fixemos $J \in \mathbb{N}$.

Então, $f\left(\bar{a}_{J}, \vec{b}_{J}\right)=0$. Além disso, $q_{J}=\left\|\vec{b}_{J}\right\| \geq\left|b_{i_{J}}\right|$, para $i=1, \ldots, m$ e $\vec{b}_{J}=\left(b_{1_{J}}, \ldots, b_{m_{J}}\right)$. Então, pela Afirmação 3 , existe $\vec{z}$ tal que $f\left(\bar{a}_{J}, q_{J}, \vec{b}_{J}, \vec{z}\right)=0$.

Logo,

$$
\left(\bar{a}_{J}, q_{J}\right) \in \pi_{n+1+2 m, n+1}[Z(g)]
$$

Seja $\left.q \in] 0, q_{J}\right]$.

Como $q_{J}=\left\|\vec{b}_{J}\right\|$ é o menor dos $\|\vec{y}\|$ tais que $f\left(\bar{a}_{J}, \vec{y}\right)=0$, logo não existe $\vec{y} \in \mathbb{R}^{m}$ tal que $\|\vec{y}\|<q_{J}$ e $f\left(\bar{a}_{J}, \vec{y}\right)=0$. Ou seja, para todo $\vec{y} \in \mathbb{R}^{m}$,

$$
\left(\|\vec{y}\|<q_{J} \text { e } f\left(\bar{a}_{J}, \vec{y}\right) \neq 0\right) \text { ou }\left(f\left(\bar{a}_{J}, \vec{y}\right)=0 \text { e }\|\vec{y}\| \geq q_{J}\right)
$$

Em ambos os casos, temos, pela Afirmação 3, que não existe $\vec{z} \in \mathbb{R}^{m}$ de sorte que $g\left(\bar{a}_{J}, q, \vec{y}, \vec{z}\right)=0$, seja qual for $\vec{y} \in \mathbb{R}^{m}$. Equivalentemente, para todo $(\vec{y}, \vec{z}) \in \mathbb{R}^{2 m}$, temos que $g\left(\bar{a}_{J}, q, \vec{y}, \vec{z}\right) \neq 0$.

Então, $\left(\bar{a}_{J}, q\right) \notin \pi_{n+1+2 m, n+1}[Z(g)]$.

Seja $\left(q_{l}\right)_{l} \in\left[0, q_{J}\right)$ tal que $q_{l} \rightarrow q_{J}$. Logo, $\left(\bar{a}_{J}, q_{l}\right) \rightarrow\left(\bar{a}_{J}, q_{J}\right)$, sendo que a sequência $\left(\bar{a}_{J}, q_{l}\right)_{l}$ está em $\mathbb{R}^{n+1} \backslash \pi_{n+1+2 m, n+1}[Z(g)]$. Assim,

$$
\left(\bar{a}_{J}, q_{J}\right) \in \overline{\mathbb{R}^{n+1} \backslash \pi_{n+1+2 m, n+1}[Z(g)]} .
$$

Desta última afirmação e de $(\dagger)$, temos que

$$
\left(\bar{a}_{J}, q_{J}\right) \in \delta\left(\pi_{n+1+2 m, n+1}[Z(g)]\right)
$$

Agora, definimos $Z^{\prime \prime \prime}=\left\{(\vec{y}, \vec{z}): g\left(\bar{a}_{J}, q_{J}, \vec{y}, \vec{z}\right)=0\right\}$ e, analogamente ao item $(1), Z^{\prime \prime \prime}$ é 
elemento de $C h(\mathcal{S})$ e tem um número finito de componentes conexas, todas estas limitadas. Logo, pelo Lema 2.4.1, $\left(\bar{a}_{J}, q_{J}\right) \in E$.

Visto que $J$ é arbitrário, segue que

$$
\left(\bar{a}_{j}, q_{j}\right) \in E \text {, para todo } j \in \mathbb{N} \text {. }
$$

Do fato de $\left(q_{j}\right)_{j}$ ser ilimitada, com $q_{j}>0$ (para todo $j$ ), concluímos que $\left(q_{j}\right)_{j}$ possui uma subsequência $\left(q_{j_{s}}\right)_{s}$ tal que $q_{j_{s}} \rightarrow+\infty$.

Considere a sequência $\left(\bar{a}_{j_{s}}, \frac{1}{q_{j_{s}}}\right)_{s}$. Então,

$$
\text { para todo } s, \quad\left(\bar{a}_{j_{s}}, \frac{1}{1 / q_{j_{s}}}\right)=\left(\bar{a}_{j_{s}}, q_{j_{s}}\right) \in E \text {. }
$$

Logo, segue, da definição de $Q$, que $\left(\bar{a}_{j_{s}}, \frac{1}{q_{j_{s}}}\right)_{s} \in Q$.

Além disso, $\frac{1}{q_{j_{s}}} \rightarrow 0$, já que $q_{j_{s}} \rightarrow+\infty$.

Portanto, $(\bar{a}, 0) \in \bar{Q}$. E, assim, $\bar{a} \in T$.

De $T \in C h(\mathcal{S})_{n}$ e das Afirmações 6 e 7, segue, portanto, o teorema.

\subsection{Condições suficientes para uma estrutura ser modelo completa e o-minimal}

Esta seção é o ponto alto deste trabalho. A nossa meta é estabelecer condições sobre uma expansão de $\overline{\mathbb{R}}$ do tipo $\langle\overline{\mathbb{R}},\{f: f \in R\}\rangle$, que sejam suficientes para obter a modelo completude da teoria da expansão. Mais precisamente, veremos que se os conjuntos definíveis, livres de quantificadores, da expansão formam uma estrutura fraca o-minimal e se o fecho topológico de um conjunto existencialmente definível for também existencialmente definível, então a estrutura $\langle\overline{\mathbb{R}},\{f: f \in R\}\rangle$ é modelo completa. Como consequência, concluímos que a coleção de todos os conjuntos definíveis é uma estrutura fraca o-minimal. Dessa forma, garantimos a o-minimalidade da estrutura. 
Antes de qualquer desenvolvimento nesta seção, atentamos para a terminologia.

Aviso. Daqui em diante, ao declararmos que uma estrutura é o-minimal no sentido modeloteórico, queremos dizer que é o-minimal nos moldes da Definição 1.2.4.

Notação. Ao longo desta seção,

- $R_{n}$ denotará uma coleção de funções $f: \mathbb{R}^{n} \rightarrow \mathbb{R}$, para cada $n$.

- $R=\bigcup_{n \in \mathbb{N}^{+}} R_{n}$.

Conjuntos definíveis na estrutura $\langle\overline{\mathbb{R}},\{f: f \in R\}\rangle$ têm o significado afirmado na Definição 1.2.1, i. e., definível (com parâmetros) por uma fórmula da linguagem em questão, não por uma Ch-fórmula.

Usaremos a notação $\phi(\bar{x}) \sim \psi(\bar{x})$ para simplificar $\langle\overline{\mathbb{R}},\{f: f \in R\}\rangle \models \forall \bar{x}(\phi(\bar{x}) \leftrightarrow \psi(\bar{x}))$.

Considere a linguagem expandida $\tilde{L}=L \cup\{f: f \in R\}$ para a estrutura $\left\langle\overline{\mathbb{R}},\{f\}_{f \in R}\right\rangle$. Então, nesta estrutura, um $\tilde{L}$-termo $t(\bar{x})$ pode ser escrito da seguinte forma $p\left(\bar{x}, f_{1}\left(\bar{x}^{(1)}\right), \ldots, f_{l}\left(\bar{x}^{(l)}\right)\right)$, de sorte que $p$ é um polinômio em $\mathbb{R}\left[z_{1}, \ldots, z_{n+l}\right], f_{1}, \ldots, f_{l} \in R$ e $\bar{x}^{(i)}$ é uma subsequência de $\bar{x}$.

Lema 2.5.1. Sejam $n \in \mathbb{N}^{+}$e $A \subseteq \mathbb{R}^{n}$ conjunto definido em $\langle\overline{\mathbb{R}},\{f: f \in R\}\rangle$ por uma $\Sigma_{1}$-fórmula $\phi(\bar{x})$. Então, existem um polinômio $p \in \mathbb{R}\left[z_{1}, \ldots, z_{m+n+l}\right]$ e funções $f_{1}, \ldots, f_{l} \in R$ tais que

$$
\phi(\bar{x}) \sim \exists \vec{y} p\left(\bar{x}, \vec{y}, f_{1}(\bar{x}, \vec{y}), \ldots, f_{l}(\bar{x}, \vec{y})\right)=0
$$

Demonstração. Seja $\phi(\bar{x})=\exists z_{1} \cdots \exists z_{m} \theta(\bar{x}, \vec{z})$ uma $\widetilde{L}$-fórmula existencial arbitrária, de modo que $\widetilde{L}=L \cup\{f: f \in R\}$ e $\theta(\bar{x}, \vec{z})$ é aberta. Então, $\theta$ pode ser colocada na forma normal disjuntiva, i. e.,

$$
\theta(\bar{x}, \vec{z}) \leftrightarrow \bigvee_{i=1}^{N} \bigwedge_{j=1}^{M} \theta_{i j}(\bar{x}, \vec{z}),
$$

tal que cada $\theta_{i j}(\bar{x}, \vec{z})$ é uma fórmula atômica ou a negação de uma fórmula atômica em $\tilde{L}$. 
Esta bi-implicação é uma fórmula válida em todas as $\tilde{L}$-estruturas, então, em particular,

$$
\theta(\bar{x}, \vec{z}) \sim \bigvee_{i=1}^{N} \bigwedge_{j=1}^{M} \theta_{i j}(\bar{x}, \vec{z})
$$

Uma fórmula atômica em $\tilde{L}$ é igual a $t_{1}=t_{2}$ ou a $t_{1}<t_{2}$, que equivale, em $\left\langle\overline{\mathbb{R}},\{f\}_{f \in R}\right\rangle$, a $t=0$ e $0<t$, respectivamente, com $t_{1}, t_{2}$ e $t \tilde{L}$-termos. Assim, podemos assumir que $\theta_{i j}(\bar{x}, \vec{z})$ é do tipo

$$
t_{i j}(\bar{x}, \vec{z})=0, t_{i j}(\bar{x}, \vec{z})>0, t_{i j}(\bar{x}, \vec{z}) \neq 0 \text { ou } t_{i j}(\bar{x}, \vec{z}) \leq 0
$$

Logo, $\theta(\bar{x}, \vec{z})$ pode ser escrito como

$$
\theta(\bar{x}, \vec{z}) \sim \bigvee_{i=1}^{N} \bigwedge_{j=1}^{M} t_{i j}(\bar{x}, \vec{z}) * 0
$$

$\operatorname{com} * \in\{=, \neq,<, \geq\}$.

Note que, para cada $i, j$, em $\langle\overline{\mathbb{R}},\{f: f \in R\}\rangle$,

$$
\begin{gathered}
t_{i j}(\bar{x}, \vec{z}) \neq 0 \sim \exists y_{i j}\left[y_{i j} \cdot t_{i j}(\bar{x}, \vec{z})-1=0\right], \\
t_{i j}(\bar{x}, \vec{z}) \leq 0 \sim \exists y_{i j}\left[t_{i j}(\bar{x}, \vec{z})+y_{i j}^{2}=0\right], \\
t_{i j}(\bar{x}, \vec{z})>0 \sim \exists y_{i j} \exists w_{i j}\left[\left(t_{i j}(\bar{x}, \vec{z})-y_{i j}^{2}=0\right) \wedge\left(w_{i j} \cdot y_{i j}-1=0\right)\right] \\
\sim \exists y_{i j} \exists w_{i j}\left[\left(t_{i j}(\bar{x}, \vec{z})-y_{i j}^{2}\right)^{2}+\left(w_{i j} \cdot y_{i j}-1\right)^{2}=0\right],
\end{gathered}
$$




$$
\begin{aligned}
t_{i j}(\bar{x}, \vec{z})=0 & \sim \exists y_{i j} \exists w_{i j}\left[\left(y_{i j} \cdot t_{i j}(\bar{x}, \vec{z})=0\right) \wedge\left(w_{i j} \cdot y_{i j}-1=0\right)\right] \\
& \sim \exists y_{i j} \exists w_{i j}\left[\left(y_{i j} \cdot t_{i j}(\bar{x}, \vec{z})\right)^{2}+\left(w_{i j} \cdot y_{i j}-1\right)^{2}=0\right] .
\end{aligned}
$$

Lembrando que um $\tilde{L}$-termo pode ser escrito como $p\left(\bar{x}, f_{1}\left(\bar{x}^{(1)}, \ldots, f_{l}\left(\bar{x}^{(l)}\right)\right)\right)$, temos, portanto,

$$
\bigvee_{i=1}^{N} \bigwedge_{j=1}^{M}\left(t_{i j}(\bar{x}, \vec{z}) * 0\right) \sim \bigvee_{i=1}^{N} \bigwedge_{j=1}^{M} \exists \tilde{y}_{i j}\left(p_{i j}\left(\bar{x}, \vec{z}, \tilde{y}_{i j}, f_{1}^{i j}, \ldots, f_{l_{i j}}^{i j}\right)=0\right)
$$

sendo que $f_{1}^{i j}, \ldots, f_{l_{i j}}^{i j} \in R$ e $p_{i j}$ é um polinômio em $\mathbb{R}$. Como as variáveis $\tilde{y}_{i j}, \bar{x}, \vec{z}$ são diferentes entre si, segue que os quantificadores comutam com disjunções e conjunções finitas. Dessa forma, podemos aceitar que

$$
\theta(\bar{x}, \vec{z})=\exists \tilde{y}_{11} \cdots \exists \tilde{y}_{1 M} \cdots \exists \tilde{y}_{N 1} \cdots \exists \tilde{y}_{N M} \bigvee_{i=1}^{N} \bigwedge_{j=1}^{M}\left(p_{i j}\left(\bar{x}, \vec{z}, \tilde{y}_{i j}, f_{1}^{i j}, \ldots, f_{l_{i j}}^{i j}\right)=0\right)
$$

Para finalizar, observamos que

$$
\bigvee_{i=1}^{N} \bigwedge_{j=1}^{M} p_{i j}=0 \sim \prod_{i=1}^{N} \sum_{j=1}^{M} p_{i j}^{2}=0
$$

e, assim, temos a equivalência

$$
\phi(\bar{x}) \leftrightarrow \exists \bar{z} \exists \tilde{y}_{11} \ldots \exists \tilde{y}_{1 M} \cdots \exists \tilde{y}_{N 1} \ldots \exists \tilde{y}_{N M} \prod_{i=1}^{N} \sum_{j=1}^{M} p_{i j}^{2}\left(\bar{x}, \vec{z}, \tilde{y}_{i j}, f_{1}^{i j}, \ldots, f_{l_{i j}}^{i j}\right)=0,
$$

válida na estrutura $\langle\overline{\mathbb{R}},\{f: f \in R\}\rangle$.

Em outros termos, esta última proposição afirma que os conjuntos $\exists_{1}$-definíveis em $\langle\overline{\mathbb{R}},\{f\}\rangle_{f \in R}$ podem ser escritos como projeções de conjuntos de zeros de funções. Como podemos notar, algumas características destes conjuntos definíveis dependem apenas de quão suave são as 
funções $f_{1}, \ldots, f_{l}{ }^{20}$ que os definem. Assim, por exemplo, se as funções $f_{1}, \ldots, f_{l}$ são contínuas, então $p\left(\bar{x}, \vec{y}, f_{1}(\bar{x}, \vec{y}), \ldots, f_{l}(\bar{x}, \vec{y})\right)$ é também contínua e, consequentemente, o conjunto $\left\{(\bar{x}, \vec{y}): p\left(\bar{x}, \vec{y}, f_{1}(\bar{x}, \vec{y}), \ldots, f_{l}(\bar{x}, \vec{y})\right)=0\right\}$ é fechado. Visto que estas caracterizações são de nosso interesse, listamos, subsequentemente, suposições sobre o conjunto de funções $R$, das quais destacaremos algumas consequências.

Suposição 2.5.2. A coleção $R$ de funções satisfaz as seguintes condições:

(R1) Cada $R_{n}$ consiste de funções $\mathcal{C}^{1}$.

(R2) A coleção dos conjuntos definíveis sem quantificadores em $\langle\overline{\mathbb{R}},\{f: f \in R\}\rangle$ satisfaz (EF5).

(R3) Se $A$ é $\exists_{1}$-definível em $\langle\overline{\mathbb{R}},\{f: f \in R\}\rangle$, então $\bar{A}$ também o é.

Definição 2.5.3. Seja $\mathcal{S}=\left\langle\mathcal{S}_{n}: n \geq 1\right\rangle$ a sequência, em que cada $\mathcal{S}_{n}$ é a coleção dos subconjuntos de $\mathbb{R}^{n}$ definíveis sem quantificadores em $\langle\overline{\mathbb{R}},\{f: f \in R\}\rangle$.

Então, $\mathcal{S}$ é uma estrutura fraca, conforme a Definição 2.1.3.

Lema 2.5.4. S satisfaz (EF1)-(EF4).

Demonstração.

(EF1) Sejam $A, B \in \mathcal{S}_{n}$ definidos pelas fórmulas abertas $\phi_{A}(\bar{x}, \vec{y})$ e $\phi_{B}(\bar{x}, \tilde{z})$, respectivamente. Logo, a conjunção $\phi_{A}(\bar{x}, \vec{y}) \wedge \phi_{B}(\bar{x}, \tilde{z})$ é uma fórmula aberta que define $A \cap B$.

(EF2) É imediato da definição de conjunto semi-algébrico.

(EF3) Considere $A \in \mathcal{S}_{n}$ e $B \in \mathcal{S}_{m}$ e sejam $\phi_{A}(\bar{x}, \hat{y})$ e $\phi_{B}(\vec{x}, \tilde{z})$ as fórmulas abertas que definem, respectivamente, tais conjuntos. Assim, a fórmula aberta $\phi_{A}(\bar{x}, \hat{y}) \wedge \phi_{B}(\vec{x}, \tilde{z})$ define $A \times B$.

(EF4) Sejam $A \in \mathcal{S}_{n}$ e $\sigma: \mathbb{R}^{n} \rightarrow \mathbb{R}^{n}$ uma bijeção linear. Então, $\sigma^{-1}: \mathbb{R}^{n} \rightarrow \mathbb{R}^{n}$ é também bijeção linear.

\footnotetext{
${ }^{20} \mathrm{Ou}$ seja, depende da classe de diferenciabilidade a que tais funções pertencem.
} 
Considere $B=\left\{e_{1}, \ldots, e_{n}\right\}$ a base canônica de $\mathbb{R}^{n}$. Então, $B_{\sigma}=\left\{\sigma^{-1}\left(e_{1}\right), \ldots, \sigma^{-1}\left(e_{n}\right)\right\}$ é uma base de $\mathbb{R}^{n}$. Podemos, portanto, expressar os elementos de $B_{\sigma}$ na base canônica $B$,

$$
\begin{array}{ccccc}
\sigma^{-1}\left(e_{1}\right)= & \lambda_{11} e_{1}+ & \cdots & +\lambda_{n 1} e_{n}= & \left(\lambda_{11}, \ldots, \lambda_{n 1}\right) \\
\vdots & \vdots & & \vdots & \vdots \\
\sigma^{-1}\left(e_{n}\right)= & \lambda_{1 n} e_{1}+ & \cdots & +\lambda_{n n} e_{n}= & \left(\lambda_{1 n}, \ldots, \lambda_{n n}\right)
\end{array}
$$

Assim, para qualquer $\bar{y} \in \mathbb{R}^{n}$, segue que

$$
\sigma^{-1}\left(y_{1}, \ldots, y_{n}\right)=\left(y_{1}, \ldots, y_{n}\right)_{B_{\sigma}}=\left(y_{1} \lambda_{11}+\cdots+y_{n} \lambda_{1 n}, \ldots, y_{1} \lambda_{n 1}+\cdots+y_{n} \lambda_{n n}\right) .
$$

Portanto,

$$
\begin{aligned}
\bar{y} \in \sigma[A] \Leftrightarrow \sigma^{-1}(\bar{y}) \in A & \Leftrightarrow\left(y_{1} \lambda_{11}+\cdots+y_{n} \lambda_{1 n}, \ldots, y_{1} \lambda_{n 1}+\cdots+y_{n} \lambda_{n n}\right) \in A \\
& \Leftrightarrow \bigwedge_{i=1}^{n}\left(x_{i}=y_{1} \lambda_{i 1}+\cdots+y_{n} \lambda_{i n}\right) \wedge \phi_{A}(\bar{x}, \tilde{b}),
\end{aligned}
$$

sendo que $\tilde{b} \in \mathbb{R}$ e $\phi_{A}$ define $A$.

Logo, $\sigma[A] \in \mathcal{S}_{n}$.

Lema 2.5.5. Se cada $R_{n}$ consiste de funcões $\mathcal{C}^{0}$, então $\mathcal{S}$ satisfaz (EF6).

Demonstração. Fixe $n \in \mathbb{N}^{+}$. Seja $A \in \mathcal{S}_{n}$. Então,

$$
A=\left\{\bar{x} \in \mathbb{R}^{n}:\langle\overline{\mathbb{R}},\{f: f \in R\}\rangle \models \phi_{A}(\bar{x}, \tilde{b})\right\}, \operatorname{com} \phi_{A}(\bar{x}, \tilde{y}) \text { aberta e } \tilde{b} \in \mathbb{R}^{k} .
$$

Pelo Lema 2.5.1, existem $p \in \mathbb{R}\left[z_{1}, \ldots, z_{m+n+l}\right]$ e funções $f_{1}, \ldots, f_{l} \in R$ tal que

$$
\phi_{A}(\bar{x}, \tilde{y}) \sim \exists \vec{y} p\left(\bar{x}, \vec{y}, f_{1}(\bar{x}, \vec{y}), \ldots, f_{l}(\bar{x}, \vec{y})\right)=0 .
$$

Portanto, $A=\left\{\bar{x} \in \mathbb{R}^{n}:\langle\overline{\mathbb{R}},\{f: f \in R\}\rangle \models \exists \vec{y} p\left(\bar{x}, \vec{y}, f_{1}(\bar{x}, \vec{y}), \ldots, f_{l}(\bar{x}, \vec{y})\right)=0\right\}$. 
Seja $h: \mathbb{R}^{n+m} \rightarrow \mathbb{R}^{m+n+l}$ dada por

$$
h=\left(i d_{m+n}, f_{1}, \ldots, f_{l}\right)
$$

em que $i d_{m+n}$ é a identidade.

Uma vez que, as funções $f_{1}, \ldots, f_{l}$ são contínuas, segue que $h$ é contínua. Logo, $f=p \circ h$ também o é.

Seja $Z(f)=\left\{(\bar{x}, \vec{y}) \in \mathbb{R}^{n+m}: f(\bar{x}, \vec{y})=0\right\}$ o conjunto dos zeros de $f$.

Então, $Z(f) \in \mathcal{S}_{n+m}$. Também, $Z(f)$ pode ser escrito como $f^{-1}[\{0\}]$. Logo, $Z(f)$ é um fechado de $\mathbb{R}^{n+m}$.

Como $A=\pi_{n+m, n}[Z(f)]$, então segue o resultado.

Como consequência imediata deste lema, temos que se (R1) vale, então $\mathcal{S}$ satisfaz (EF6).

Assim, se ambas as condições (R1) e (R2) valem, então $\mathcal{S}$ é uma estrutura fraca o-minimal, de acordo com a Definição 2.1.6.

Já a condição $(\mathrm{R} 3)$ acarreta o seguinte fato: a coleção $C h(\mathcal{S})$ corresponde a dos conjuntos $\exists_{1}$-definíveis em $\langle\overline{\mathbb{R}},\{f: f \in R\}\rangle$, conforme mostra o próximo resultado.

Lema 2.5.6. Suponha que a condição (R3) valha. Então, $C h(\mathcal{S})=\mathcal{S}^{p r}$.

Demonstração. Em primeiro lugar, lembremos, da Definição 2.1.10, que

$$
C h(\mathcal{S})=\bigcup_{i \in \mathbb{N}} \mathcal{S}^{i}, \operatorname{com} \mathcal{S}^{i+1}=\left(\left(\left(\mathcal{S}^{i}\right)^{u}\right)^{p r}\right)^{c l} \text { e } \mathcal{S}^{0}=\mathcal{S}
$$

Basta, portanto, mostrarmos que $\mathcal{S}^{i}=\mathcal{S}^{p r}$, para todo $i \in \mathbb{N}^{+}$. Isto será feito por indução em $i$.

Vejamos que $\mathcal{S}^{1}=\mathcal{S}^{p r}$

Com efeito, $\mathcal{S}^{1}=\left(\left(\mathcal{S}^{u}\right)^{p r}\right)^{c l}$. Considere a afirmação a seguir, 
AFIRMAÇÃO $1 . \mathcal{S}^{u}=\mathcal{S}$.

Prova da Afirmação. Fixe $n \geq 1$. Então, diretamente da definição de $\mathcal{S}_{n}^{u}$, segue que $\mathcal{S}_{n} \subseteq \mathcal{S}_{n}^{u}$.

Por outro lado, dado $A \in \mathcal{S}_{n}^{u}$, temos que

$$
A=\bigcup_{i=1}^{p} A_{i}
$$

$\operatorname{com} p \geq 1$ e $A_{i}, \ldots, A_{p} \in \mathcal{S}_{n}$.

Seja $\phi_{A_{i}}$ a fórmula aberta que define $A_{i}$. Então,

$$
\bigvee_{i=1}^{p} \phi_{A_{i}}
$$

define $A$ e é aberta.

Assim, $\mathcal{S}_{n}^{u} \subseteq \mathcal{S}_{n}$.

Portanto, $\mathcal{S}^{1}=\left(\mathcal{S}^{p r}\right)^{c l}$.

Observemos, agora, a asserção subsequente.

AFIRMAÇÃO 2. $\left(\mathcal{S}^{p r}\right)^{c l}=\mathcal{S}^{p r}$.

Prova da Afirmação. Seja $A \in\left(\mathcal{S}^{p r}\right)^{c l}$. Então,

$$
A=A_{0} \cap \bigcap_{i=1}^{p} \bar{A}_{i},
$$

$\operatorname{com} p \geq 1$ e $A_{0}, A_{1}, \ldots, A_{p} \in \mathcal{S}^{p r}$.

Pela condição (R3), $\bar{A}_{1}, \ldots, \bar{A}_{p} \in \mathcal{S}^{p r}$. Pelo Lema 2.5.4, $\mathcal{S}$ goza das propriedades (EF1)(EF4) e, então, pelo Lema 2.1.9,

$$
A=A_{0} \cap \bigcap_{i=1}^{p} \bar{A}_{i} \in \mathcal{S}^{p r} .
$$


Assim, $\left(\mathcal{S}^{p r}\right)^{c l} \subseteq \mathcal{S}^{p r}$

Por outro lado, fixando $n \in \mathbb{N}^{+}$e $A \in \mathcal{S}_{n}^{p r}$, temos que $A=\pi_{m, n}[B]$, para algum $m \geq n$ e $B \in \mathcal{S}_{m}$.

Assim, $A$ pode ser escrito da forma

$$
A=A_{0} \cap \bigcap_{i=1}^{p} \bar{A}_{i},
$$

em que $A_{0}=\pi_{m, n}[B]$ e $p=0$.

Portanto, $A \in\left(\mathcal{S}^{p r}\right)^{c l} \mathrm{e}$, assim, $\mathcal{S}^{p r} \subseteq\left(\mathcal{S}^{p r}\right)^{c l}$.

Como consequência desta afirmação, temos que $\mathcal{S}^{1}=\mathcal{S}^{p r}$.

Agora, suponha que $\mathcal{S}^{i}=\mathcal{S}^{p r}$. Vejamos, então, que $\mathcal{S}^{i+1}=\mathcal{S}^{p r}$.

Por definição, $\mathcal{S}^{i+1}=\left(\left(\left(\mathcal{S}^{i}\right)^{u}\right)^{p r}\right)^{c l}$. Logo, pela hipótese de indução, $\mathcal{S}^{i+1}=\left(\left(\left(\mathcal{S}^{p r}\right)^{u}\right)^{p r}\right)^{c l}$. Considere, então, o seguinte resultado.

AFIRMAÇÃO 3. $\left(\mathcal{S}^{p r}\right)^{u}=\left(\mathcal{S}^{u}\right)^{p r}$.

Prova da Afirmação. Fixe $n \in \mathbb{N}^{+}$.

Seja $A \in\left(\mathcal{S}^{p r}\right)_{n}^{u}$. Então,

$$
A=\bigcup_{i=1}^{p} A_{i},
$$

$\operatorname{com} p \geq 1$ e $A_{1}, \ldots, A_{p} \in \mathcal{S}_{n}^{p r}$

Consequentemente,

$$
A_{i}=\pi_{m_{i}, n}\left[B_{i}\right]
$$

sendo que $m_{i} \geq n$ e $B_{i} \in \mathcal{S}_{m_{i}}$, para todo $i$.

Tome $m=\max \left\{m_{1}, \ldots, m_{p}\right\}$. Então,

$$
B_{i} \times \mathbb{R}^{m-m_{i}} \in \mathcal{S}_{m},
$$


para qualquer $i$.

Uma vez que $\pi_{m_{i}, n}\left[B_{i}\right]=\pi_{m, n}\left[B_{i} \times \mathbb{R}^{m-m_{i}}\right]$, então

$$
\pi_{m, n}\left[\bigcup_{i=1}^{p}\left(B_{i} \times \mathbb{R}^{m-m_{i}}\right)\right]=\bigcup_{i=1}^{p} \pi_{m, n}\left[B_{i} \times \mathbb{R}^{m-m_{i}}\right]=\bigcup_{i=1}^{p} \pi_{m_{i}, n}\left[B_{i}\right]=\bigcup_{i=1}^{p} A_{i}=A
$$

Portanto, $A \in\left(\mathcal{S}^{u}\right)_{n}^{p r}$.

Inversamente, dado $A \in\left(\mathcal{S}^{u}\right)_{n}^{p r}$, temos que

$$
A=\pi_{m, n}[B]
$$

$\operatorname{com} m \geq n$ e $B \in \mathcal{S}_{m}^{u}$

Consequentemente,

$$
B=\bigcup_{i=1}^{p} D_{i}
$$

de sorte que $p \geq 1$ e $D_{i} \in \mathcal{S}_{m}$.

Logo,

$$
A=\pi_{m, n}\left[\bigcup_{i=1}^{p} D_{i}\right]=\bigcup_{i=1}^{p} \pi_{m, n}\left[D_{i}\right]
$$

Assim, $A \in\left(\mathcal{S}^{p r}\right)_{n}^{u}$.

Portanto, $\mathcal{S}^{i+1}=\left(\left(\left(\mathcal{S}^{u}\right)^{p r}\right)^{p r}\right)^{c l}$. Por conta da Afirmação 1, temos que $\mathcal{S}^{i+1}=\left(\left(\mathcal{S}^{p r}\right)^{p r}\right)^{c l}$. Além disso, $\left(\mathcal{S}^{p r}\right)^{p r}=\mathcal{S}^{p r}$. Logo, $\mathcal{S}^{i+1}=\left(\mathcal{S}^{p r}\right)^{c l}$. Finalmente, pela Afirmação 2, temos que $\left(\mathcal{S}^{p r}\right)^{c l}=\mathcal{S}^{p r}$.

Por conseguinte, $\mathcal{S}^{i+1}=\mathcal{S}^{p r}$.

Finalmente, juntando (R1), (R2) e (R3) obtemos a modelo completude da expansão $\langle\overline{\mathbb{R}},\{f: f \in R\}\rangle$.

Teorema 2.5.7. Considere a Suposição 2.5.2 válida. Então, Th( $\langle\overline{\mathbb{R}},\{f: f \in R\}\rangle)$ é modelo 
completa.

Demonstração. Demonstraremos o item (d) do Teorema 1.3.5.

Para isso, considere a seguinte afirmação.

AFIRMAÇÃO. Os conjuntos $\exists_{1}$-definíveis em $\langle\overline{\mathbb{R}},\{f: f \in R\}\rangle$ são fechados sob complementos.

Prova da Afirmação. Sejam $A, B \subseteq \mathbb{R}^{n}$ definidos, em $\langle\overline{\mathbb{R}},\{f: f \in R\}\rangle$, por fórmulas existenciais da linguagem $\tilde{L}=L \cup\{f: f \in R\}$.

Pelo Lema 2.5.1, $A$ e $B$ podem ser expressos na forma

$$
\{\bar{x}: \exists \vec{y} f(\bar{x}, \vec{y})=0\}
$$

de modo que $f$ é uma função $\mathcal{C}^{1}$ e $G r(f) \in \mathcal{S}$.

Uma vez que (R3) é válida, então, pelo Lema 2.5.6, segue que $\mathcal{S}^{p r}=C h(\mathcal{S})$. Logo, $A, B \in C h(\mathcal{S})$.

Agora, visto que $(\mathrm{R} 1)$ e $(\mathrm{R} 2)$ valem, então $\mathcal{S}$ é uma estrutura fraca o-minimal. Desta forma, pelo Teorema 2.4.2, existe $T \in C h(\mathcal{S})$ tal que $\delta(A) \subseteq T$ e $\stackrel{\circ}{T}=\emptyset$. Em outros termos, a Suposição 2.2.6 é válida. Por conseguinte, o Teorema da Decomposição Celular ( $i$. e., Teorema 2.2.7) fica estabelecido para $C h(\mathcal{S})$.

Assim, pelo TDC, existe $\mathcal{E}=\left\{C_{1}, \ldots, C_{l}\right\}$, decomposição de $\mathbb{R}^{n}$ que particiona $A$ e $B$.

Considere, agora, os seguintes conjuntos finitos de índices

$$
I_{A}^{+}=\left\{j \leq l: C_{j} \subseteq A\right\} \text { e } I_{A}^{-}=\left\{j \leq l: C_{j} \cap A=\emptyset\right\}
$$

e

$$
I_{B}^{+}=\left\{j \leq l: C_{j} \subseteq B\right\} \text { e } I_{B}^{-}=\left\{j \leq l: C_{j} \cap B=\emptyset\right\} .
$$

Então,

$$
A \backslash B=\bigcup\left\{C_{j}: j \in I_{A}^{+} \cap I_{B}^{-}\right\}
$$


Uma vez que $C h(\mathcal{S})$ é fechado por reunião finita, segue que $A \backslash B \in C h(\mathcal{S})$.

Novamente, como $C h(\mathcal{S})=\mathcal{S}^{p r}$, então $A \backslash B \in \mathcal{S}^{p r}$. E, assim, segue a afirmação.

Considere, agora, uma fórmula $\beta(\bar{x})$ da linguagem $\tilde{L}$. Então, pelo Teorema 1.1.9, existe uma fórmula aberta $\psi(\bar{x}, \vec{y})$ tal que

$$
\vdash \beta(\bar{x}) \leftrightarrow Q_{m} y_{m} \cdots Q_{1} y_{1} \psi(\bar{x}, \vec{y})
$$

de forma que $Q_{i} \in\{\exists, \forall\}$.

É suficiente, então, mostrar por indução em $m$, que $Q_{m} y_{m} \cdots Q_{1} y_{1} \psi(\bar{x}, \vec{y})$ é equivalente, em $\langle\overline{\mathbb{R}},\{f: f \in R\}\rangle$, a uma fórmula existencial.

De fato, o caso $m=0$ é trivial, já que $\psi(\bar{x}, \vec{y})$ é, em particular, uma fórmula existencial.

Suponha $m>0$ e que o resultado valha para todo $0 \leq j<m$.

Então, para todo $\bar{x} \in \mathbb{R}$,

$$
\langle\overline{\mathbb{R}},\{f: f \in R\}\rangle \models Q_{m-1} y_{m-1} \cdots Q_{1} y_{1} \psi(\bar{x}, \vec{y}) \leftrightarrow \exists \tilde{z} \theta\left(\bar{x}, y_{m}, \tilde{z}\right)
$$

$\operatorname{com} \theta$ aberta.

Se $Q_{m}=\exists$, então

$$
\langle\overline{\mathbb{R}},\{f: f \in R\}\rangle \models \exists y_{m} Q_{m-1} y_{m-1} \cdots Q_{1} y_{1} \psi(\bar{x}, \vec{y}) \leftrightarrow \exists y_{m} \exists \tilde{z} \theta\left(\bar{x}, y_{m}, \tilde{z}\right)
$$

E, portanto, segue o resultado.

Se $Q_{m}=\forall$, então

$$
\langle\overline{\mathbb{R}},\{f: f \in R\}\rangle \models \forall y_{m} Q_{m-1} y_{m-1} \cdots Q_{1} y_{1} \psi(\bar{x}, \vec{y}) \leftrightarrow \forall y_{m} \exists \tilde{z} \theta\left(\bar{x}, y_{m}, \tilde{z}\right)
$$

Note que,

$$
\langle\overline{\mathbb{R}},\{f: f \in R\}\rangle \models \forall y_{m} \exists \tilde{z} \theta(\bar{x}, \tilde{z}) \leftrightarrow \neg \exists y_{m} \neg \exists \tilde{z} \theta\left(\bar{x}, y_{m}, \tilde{z}\right) .
$$


Além disso, $\neg \exists \tilde{z} \theta\left(\bar{x}, y_{m}, \tilde{z}\right)$ define o complementar, em relação a $\mathbb{R}^{n}$, do conjunto definido por $\exists \tilde{z} \theta\left(\bar{x}, y_{m}, \tilde{z}\right)$.

Logo, pela Afirmação, temos que $\neg \exists \tilde{z} \theta\left(\bar{x}, y_{m}, \tilde{z}\right)$ é equivalente, em $\langle\overline{\mathbb{R}},\{f: f \in R\}\rangle$, a uma fórmula existencial $\exists \hat{w} \theta_{1}\left(\bar{x}, y_{m}, \hat{w}\right)$.

Consequentemente,

$$
\langle\overline{\mathbb{R}},\{f: f \in R\}\rangle \mid \exists y_{m} \neg \exists \tilde{z} \theta\left(\bar{x}, y_{m}, \tilde{z}\right) \leftrightarrow \exists y_{m} \exists \hat{w} \theta_{1}\left(\bar{x}, y_{m}, \hat{w}\right) .
$$

De maneira análoga, segue, novamente pela Afirmação, que

$$
\langle\overline{\mathbb{R}},\{f: f \in R\}\rangle \models \neg\left(\exists y_{m} \exists \hat{w} \theta_{1}\left(\bar{x}, y_{m}, \hat{w}\right)\right) \leftrightarrow \exists \check{t} \theta_{2}(\bar{x}, \check{t}),
$$

$\operatorname{com} \theta_{2}$ aberta.

Portanto,

$$
\langle\overline{\mathbb{R}},\{f\}\rangle_{f \in R} \models \forall y_{m} Q_{m-1} y_{m-1} \cdots Q_{1} y_{1} \psi(\bar{x}, \vec{y}) \leftrightarrow \exists \check{t} \theta_{2}(\bar{x}, \check{t}),
$$

para todo $\bar{x} \in \mathbb{R}$.

A o-minimalidade (no sentido modelo-teórico) de $\langle\overline{\mathbb{R}},\{f: f \in R\}\rangle$ segue, então, facilmente.

Corolário 2.5.8. Suponha que as condições (R1)-(R3) sejam satisfeitas. Então, a estrutura $\langle\overline{\mathbb{R}},\{f: f \in R\}\rangle$ é o-minimal (no sentido modelo-teórico).

Demonstração. Seja $A \subseteq \mathbb{R}$ definido por $\phi_{A}$, i. e. $A=\left\{x \in \mathbb{R}:\langle\overline{\mathbb{R}},\{f: f \in R\}\rangle \models \phi_{A}(x)\right\}$. Então, pelo Teorema 2.5.7, $T h(\langle\overline{\mathbb{R}},\{f: f \in R\}\rangle)$ é modelo completa. Logo, pela definição, existe $\psi$ aberta de sorte que

$$
T h(\langle\overline{\mathbb{R}},\{f: f \in R\}\rangle) \vdash \phi_{A} \leftrightarrow \exists \vec{y} \psi
$$


Logo,

$$
\langle\overline{\mathbb{R}},\{f: f \in R\}\rangle \models \forall \bar{x}\left(\phi_{A}(\bar{x}) \leftrightarrow \exists \vec{y} \psi(\bar{x}, \vec{y})\right),
$$

ou seja

$$
A=\{x \in \mathbb{R}:\langle\overline{\mathbb{R}},\{f: f \in R\}\rangle \models \exists \vec{y} \psi(\bar{x}, \vec{y}))\}
$$

Em palavras, $A$ pode ser escrito como um conjunto $\exists_{1}$-definível.

Assim, pelo Lema 2.5.6, $A \in C h(\mathcal{S})$. Portanto, por (EF5), $A=A \cap \mathbb{R}$ é união finita de intervalos e pontos.

Como conclusão desta seção, podemos afirmar, com base no Teorema 2.5.7, que a tentativa em obter a modelo completude de uma expansão, por funções, do corpo ordenado dos reais e, consequentemente, sua o-minimalidade, se reduz a averiguar se a expansão goza das propriedades (R1)-(R3). 


\section{Capítulo 3}

\section{Condições alternativas para modelo completude}

\subsection{Condições adicionais para uma estrutura ser modelo completa}

O propósito deste capítulo é explorar outras condições além das (R1)-(R3), mencionadas na última parte do capítulo anterior, que sejam suficientes para lograrmos a modelo completude de uma expansão de $\overline{\mathbb{R}}$.

Lema 3.1.1. Todo conjunto definível numa expansão o-minimal (no sentido modelo-teórico) de $\overline{\mathbb{R}}$ satisfaz (EF5).

Demonstração. Seja $A$ um conjunto definido (com parâmetros) por $\phi_{A}(\bar{x}, \tilde{a})$ numa expansão o-minimal $M$ de $\overline{\mathbb{R}}$.

Seja $p(\bar{x}, \vec{y}) \in \mathbb{Z}[\bar{x}, \vec{y}]$ o polinômio com a propriedade:

se $Z \subseteq \mathbb{R}^{n}$ é afim, então existe $\vec{b} \in \mathbb{R}^{m}$ tal que $Z=\{\bar{x}: p(\bar{x}, \vec{b})=0\}$,

conforme o comentário após a Definição 2.1.4.

Considere $\phi(\bar{x}, \tilde{a}, \vec{y})=\left[\phi_{A}(\bar{x}, \tilde{a}) \wedge(p(\bar{x}, \vec{y})=0)\right]$. Então, pelo Teorema 0.3 (pág. 593) em [12], existe $N \in \mathbb{N}$ tal que

para todo $\vec{b} \in \mathbb{R}^{m},\{\bar{x}: M \models \phi(\bar{x}, \tilde{a}, \vec{b})\}$ tem no máximo $N$ componentes conexas. 
Em outros termos, para todo $Z \subseteq \mathbb{R}^{n}$ afim, $A \cap Z$ tem no máximo $N$ componentes conexas.

Note que, o Lema 3.1.1 garante a validade de (R2). Assim, juntamente com o Teorema 2.5.7, qualquer estrutura o-minimal $\langle\overline{\mathbb{R}},\{f: f \in R\}\rangle$ que satisfaz (R1) e (R3) é modelo completa. Isto pode ter algum proveito ao analisarmos a modelo completude de um reduto. Em geral, redutos de estruturas modelo completas não são modelo completas. Contudo, redutos de estruturas o-minimais são, obviamente, o-minimais. Caminharemos, portanto, na direção de determinar condições alternativas a (R3).

Notação. Suponha $f \in R_{n}$ e $\sigma:\{1, \ldots, n\} \rightarrow\{-\infty, 0,+\infty\}$. Seja $I_{\sigma} \subseteq[-1,1]^{n}$ definido por

$$
I_{\sigma}=I_{\sigma(1)} \times \cdots \times I_{\sigma(n)},
$$

de sorte que $I_{-\infty}=\left[-1,0\left[, I_{0}=[-1,1]\right.\right.$ e $\left.\left.I_{+\infty}=\right] 0,1\right]$.

Então, para cada $\sigma$, definimos $f_{\sigma}: I_{\sigma} \rightarrow \mathbb{R}$ por

$$
f_{\sigma}(\bar{x})=f\left(\bar{x}_{\sigma}\right),
$$

sendo que

$$
\bar{x}_{\sigma}=\left(\sigma_{1}(\bar{x}), \ldots, \sigma_{n}(\bar{x})\right) \text { e } \sigma_{i}(\bar{x})=\left\{\begin{array}{cc}
x_{i} & \text { se } \sigma(i)=0 \\
\frac{1}{x_{i}} & \text { se } \sigma(i) \neq 0
\end{array} .\right.
$$

A seguir, listamos todas as condições sobre $R$ nas quais estaremos interessados subsequentemente.

(R1) Cada $R_{n}$ consiste de funções $\mathcal{C}^{1}$.

(R2) A coleção dos conjuntos definíveis sem quantificadores em $\langle\overline{\mathbb{R}},\{f: f \in R\}\rangle$ satisfaz (EF5). 
(R3) Se $A$ é $\exists_{1}$-definível em $\langle\overline{\mathbb{R}},\{f: f \in R\}\rangle$, então $\bar{A}$ também o é.

(R4) Seja $W \subseteq[-1,1]^{n}$ um conjunto da forma

$$
W=\left\{\bar{x} \in[-1,1]^{n}:(f(\bar{x})=0) \wedge \bigwedge_{\sigma(i)=+\infty}\left(x_{i}>0\right) \wedge \bigwedge_{\sigma(i)=-\infty}\left(x_{i}<0\right)\right\}
$$

em que $f(\bar{x})$ é da forma $p\left(\bar{x}, f_{1}(\bar{x}), \ldots, f_{l}(\bar{x})\right)$, com $p \in \mathbb{R}\left[z_{1}, \ldots, z_{n+l}\right] ; f_{1}, \ldots, f_{l} \in R$ e $\sigma:\{1, \ldots, n\} \rightarrow\{-\infty, 0,+\infty\}$.

Então, $\bar{W}$ é $\exists_{1}$-definível em $\langle\overline{\mathbb{R}},\{f: f \in R\}\rangle$.

(R5) Sejam $f \in R_{n}$ e $\sigma:\{1, \ldots, n\} \rightarrow\{-\infty, 0,+\infty\}$. Então, existe $h \in R_{n}$ tal que $h \uparrow I_{\sigma}=f_{\sigma}$.

(R6a) Todas as funções em $R$ são $\mathcal{C}^{\infty}$.

(R6b) $\langle\overline{\mathbb{R}},\{f: f \in R\}\rangle$ é polinomialmente limitada, $i$. e. para cada função definível $f: \mathbb{R} \rightarrow$ $\mathbb{R}$, existem $a>0$ e $p \in \mathbb{N}$ tal que

$$
|f(x)|<x^{p} \text {, para todo } x>a \text {. }
$$

(R6c) $R$ é fechado sob a derivação parcial, $i$. e e. se $f \in R_{n}$, então $\frac{\partial f}{\partial x_{i}} \in R_{n}$, para $i \in\{1, \ldots, n\}$.

Note que, (R4) segue da afirmação: se $A$ é definível sem quantificadores em $\left\langle\overline{\mathbb{R}},\{f\}_{f \in R}\right\rangle$, então $\bar{A}$ também o é. Apresentamos a condição (R4) nesta forma "mais complicada", numa tentativa de afirmar os resultados finais da maneira mais forte possível.

Estaremos interessados apenas no caso em que (R1) vale. Portanto, o fato da condição (R5) valer para cada $f \in R_{n}$ implica que $f_{\sigma}$ deve ser sempre estendível a uma função $\mathcal{C}^{1}$ com domínio $\mathbb{R}^{n}$.

Aviso. Quando nos referirmos à conjunção das condições (R6a),(R6b) e (R6c), usaremos apenas (R6).

No que segue, estaremos assumindo que (R1) e (R2) valem. 
Mostraremos, primeiro, que (R3) pode ser enfraquecido para (R4), caso (R5) valha. Posteriormente, mostraremos que se (R6) vale, então (R4) vale.

Teorema 3.1.2. Suponha que (R1), (R2), (R4) e (R5) valham. Então, Th( $\langle\overline{\mathbb{R}},\{f: f \in R\}\rangle)$ é modelo completa.

Demonstração. Pelo Teorema 2.5.7, é suficiente mostrar que (R3) vale.

Com efeito, seja $A \subseteq \mathbb{R}^{m}$ um conjunto $\exists_{1}$-definível (em $\langle\overline{\mathbb{R}},\{f: f \in R\}\rangle$ ). Pelo Lema 2.5.1, $A$ pode ser escrito na forma $\pi_{n, m}[B]$, de modo que

$$
B=\left\{\bar{x}: p\left(\bar{x}, f_{1}(\bar{x}), \ldots, f_{l}(\bar{x})\right)=0\right\}
$$

$\operatorname{com} f_{1}, \ldots, f_{l} \in R$ e $p \in \mathbb{R}\left[z_{1}, \ldots, z_{n+l}\right]$.

Considere os intervalos

$$
\begin{aligned}
& I_{-\infty}=\left[-1,0\left[\quad J_{-\infty}=\right]-\infty,-1\right] \\
& I_{0}=[-1,1] \quad \text { e } \quad J_{0}=[-1,1] \\
& \left.\left.I_{+\infty}=\right] 0,1\right] \quad J_{-\infty}=[1,+\infty[\text {. }
\end{aligned}
$$

Fixe $\sigma:\{1, \ldots, n\} \rightarrow\{-\infty, 0,+\infty\}$. Então, definimos $B_{\sigma}$ por

$$
B_{\sigma}=B \cap\left(J_{\sigma(1)} \times \cdots \times J_{\sigma(n)}\right) .
$$

E, definimos $B_{\sigma}^{*}$ por

$$
B_{\sigma}^{*}=\left\{\bar{x} \in I_{\sigma}: p\left(\bar{x}_{\sigma}, f_{1}\left(\bar{x}_{\sigma}\right), \ldots, f_{l}\left(\bar{x}_{\sigma}\right)\right)=0\right\}
$$

Mas, $f_{i}\left(\bar{x}_{\sigma}\right)=f_{i \sigma}(\bar{x})$, para $i=1, \ldots, l$ e todo $\bar{x} \in I_{\sigma}$. Logo, por (R5), existem $h_{1}, \ldots, h_{l}$ em $R$ tais que

$$
h_{i}(\bar{x})=f_{i \sigma}(\bar{x})=f_{i}\left(\bar{x}_{\sigma}\right), \text { para todo } \bar{x} \in I_{\sigma} .
$$


Dessa forma, podemos reescrever $B_{\sigma}^{*}$ como

$$
B_{\sigma}^{*}=\left\{\bar{x} \in I_{\sigma}: p\left(\bar{x}_{\sigma}, h_{1}(\bar{x}), \ldots, h_{l}(\bar{x})\right)=0\right\}
$$

Uma vez que o polinômio $p$ pode ser escrito na forma

$$
p=\sum a_{i_{1} \cdots i_{n+l}} z_{1}^{i_{1}} \cdots z_{n}^{i_{n}} z_{n+1}^{i_{n+1}} \cdots z_{n+l}^{i_{n+l}}
$$

temos,

$$
p\left(\sigma_{1}(\bar{x}), \ldots, \sigma_{n}(\bar{x}), h_{1}(\bar{x}), \ldots, h_{l}(\bar{x})\right)=0
$$

se, e somente se

$$
\sum a_{i_{1} \cdots i_{n+l}} \sigma_{1}^{i_{1}}(\bar{x}) \cdots \sigma_{n}^{i_{n}}(\bar{x}) h_{n+1}^{i_{n+1}}(\bar{x}) \cdots h_{n+l}^{i_{n+l}}(\bar{x})=0
$$

Pela definição de $\sigma_{i}(\bar{x})$, temos que este é igual ou a $x_{i}$ ou a $\frac{1}{x_{i}}, \operatorname{com} \bar{x}=\left(x_{1}, \ldots, x_{i}, \ldots, x_{n}\right)$.

Sejam $j_{1}, \ldots, j_{m} \in\{1, \ldots, n\}$ tais que

$$
\sigma_{j_{1}}(\bar{x})=\frac{1}{x_{j_{1}}}, \ldots, \sigma_{j_{m}}(\bar{x})=\frac{1}{x_{j_{m}}} .
$$

Então, multiplicando pelo produtório, em todas as configurações $i_{1} \cdots i_{n+l}$, de $1 /\left[\sigma_{j_{1}}^{i_{j_{1}}}(\bar{x}) \cdots \sigma_{j_{m}}^{i_{j_{m}}}(\bar{x})\right]$ ambos os lados da igualdade

$$
\sum a_{i_{1} \cdots i_{n+l}} \sigma_{1}^{i_{1}}(\bar{x}) \cdots \sigma_{n}^{i_{n}}(\bar{x}) h_{n+1}^{i_{n+1}}(\bar{x}) \cdots h_{n+l}^{i_{n+l}}(\bar{x})=0 .
$$

chegamos a

$$
\sum a_{i_{1} \cdots i_{n+l}} x_{k_{1}}^{i_{k_{1}}} \cdots x_{k_{p}}^{i_{k_{p}}} x_{j_{1}}^{\tilde{i}_{j_{1}}} \cdots x_{j_{m}}^{\tilde{i}_{j_{m}}} h_{n+1}^{i_{n+1}}(\bar{x}) \cdots h_{n+l}^{i_{n+l}}(\bar{x})=0
$$

em que $\left\{k_{1}, \ldots, k_{p}\right\}=\{1, \ldots, n\} \backslash\left\{j_{1}, \ldots, j_{m}\right\}$ e $\tilde{i}_{j_{s}}=\sum i_{j_{s}}$ (somatória sobre todas as configurações distintas de $\left.i_{1} \cdots i_{n+l}\right)$, para $s=1, \ldots, m$.

Assim, obtemos um polinômio $q \in \mathbb{R}\left[z_{1}, \ldots, z_{n+l}\right]$ tal que $q\left(\bar{x}, h_{1}(\bar{x}), \ldots, h_{l}(\bar{x})\right)=0$. 
Logo,

$$
B_{\sigma}^{*}=\left\{\bar{x} \in I_{\sigma}: q\left(\bar{x}, h_{1}(\bar{x}), \ldots, h_{l}(\bar{x})\right)=0\right\} .
$$

Como $\bar{x} \in I_{\sigma}$, então pela definição de $I_{\sigma}$, temos

$$
x_{i}<0, \text { sempre que } x_{i} \in I_{\sigma(i)} \text { e } \sigma(i)=-\infty
$$

e

$$
x_{i}>0, \text { sempre que } x_{i} \in I_{\sigma(i)} \text { e } \sigma(i)=+\infty \text {. }
$$

Portanto,

$$
B_{\sigma}^{*}=\left\{\bar{x} \in[-1,1]^{n}: q\left(\bar{x}, h_{1}(\bar{x}), \ldots, h_{l}(\bar{x})\right)=0 \wedge \bigwedge_{\sigma(i)=+\infty}\left(x_{i}>0\right) \wedge \bigwedge_{\sigma(i)=-\infty}\left(x_{i}<0\right)\right\}
$$

Logo, por (R4), $\overline{B_{\sigma}^{*}}$ é um conjunto $\exists_{1}$-definível.

Seja $\psi(\bar{x})$ a fórmula que define $\overline{B_{\sigma}^{*}}$. Então,

$$
\exists y_{1} \cdots \exists y_{n-m} \psi\left(\bar{x}, y_{1}, \ldots, y_{n-m}\right)
$$

define $\pi_{n, m}\left[\overline{B_{\sigma}^{*}}\right]$.

Agora, considere

$$
A_{\sigma}=\left\{\vec{x}: \exists \vec{y}\left(\bigwedge_{\sigma(i) \neq 0}\left(x_{i} y_{i}=1\right) \wedge \bigwedge_{\sigma(i)=0}\left(x_{i}=y_{i}\right) \wedge \vec{y} \in \pi_{n, m}\left[\overline{B_{\sigma}^{*}}\right]\right)\right\}
$$

Seja, também, $\Sigma$ o conjunto de todas as funções $\sigma:\{1, \ldots, n\} \rightarrow\{-\infty, 0,+\infty\}$. AfIRMAÇÃo. $\bar{A}=\bigcup_{\sigma \in \Sigma} A_{\sigma}$

Prova da Afirmação. Vejamos que $A_{\sigma} \subseteq \bar{A}$, para todo $\sigma \in \Sigma$.

Fixe $\sigma \in \Sigma$. Seja $\vec{x} \in A_{\sigma}$. Logo, existe $\vec{y}$ tal que 


$$
(*) \quad y_{i}= \begin{cases}x_{i}, & \text { se } \sigma(i)=0 \\ \frac{1}{x_{i}}, & \text { se } \sigma(i) \neq 0\end{cases}
$$

e $\vec{y} \in \pi_{n, m}\left[\overline{B_{\sigma}^{*}}\right]$.

Note que, de $(*)$, obtemos

$$
(* *) \quad \vec{y}_{\sigma}=\left(\sigma_{1}(\vec{y}), \ldots, \sigma_{m}(\vec{y})\right)=\left(x_{1}, \ldots, x_{m}\right)=\vec{x} .
$$

De $\vec{y} \in \pi_{n, m}\left[\overline{B_{\sigma}^{*}}\right]$, segue que existe $\hat{y}=\left(y_{1}, \ldots, y_{n-m}\right) \in \mathbb{R}^{n-m}$ tal que $\bar{y}=(\vec{y}, \hat{y}) \in \overline{B_{\sigma}^{*}}$. Então, existe uma sequência $\left(\bar{y}_{j}\right)_{j}=\left(\vec{y}_{j}, \hat{y}_{j}\right)_{j}$ em $B_{\sigma}^{*}$ tal que $\bar{y}_{j} \rightarrow \bar{y}$. Consequentemente,

$$
\bar{y}_{j} \in I_{\sigma} \text { e } f\left(\bar{y}_{j \sigma}\right)=p\left(\bar{y}_{j \sigma}, f_{1}\left(\bar{y}_{j \sigma}\right), \ldots, f_{l}\left(\bar{y}_{j \sigma}\right)\right)=0, \text { para todo } j .
$$

Desta última afirmação, segue que

$$
(* * *) \quad\left(\bar{y}_{j \sigma}\right)_{j} \in B
$$

Vejamos, agora, que se $\bar{y}_{j} \rightarrow \bar{y}$, então $\vec{y}_{j \sigma} \rightarrow \vec{y}_{\sigma}$.

De fato, pela definição, $\vec{y}_{j \sigma}=\left(\sigma_{1}\left(\bar{y}_{j}\right), \ldots, \sigma_{n}\left(\bar{y}_{j}\right)\right)$, com

$$
\sigma_{i}\left(\vec{y}_{j}\right)=\left\{\begin{array}{ll}
y_{j i}, & \text { se } \sigma(i)=0 \\
\frac{1}{y_{j i}}, & \text { se } \sigma(i) \neq 0
\end{array} .\right.
$$

Assim, para todo $i \in\{1, \ldots, m\}, \sigma_{i}\left(\vec{y}_{j}\right) \rightarrow \sigma_{i}(\vec{y})$.

Com efeito, fixemos $i \in\{1, \ldots, m\}$. Considere a sequência $\left(\sigma_{i}\left(\vec{y}_{j}\right)\right)_{j}$.

Se $\sigma(i)=0$, então $\sigma_{i}\left(\vec{y}_{j}\right)=y_{j i}$, para todo $j$. Logo, pela hipótese $\left(i . e ., \bar{y}_{j} \rightarrow \bar{y}\right.$, que tem como implicação $\left.\vec{y}_{j} \rightarrow \vec{y}\right)$, temos que $y_{j i} \rightarrow y_{i}\left(=\sigma_{i}(\vec{y})\right)$. 
Se $\sigma(i) \neq 0$, então $\sigma_{i}\left(\vec{y}_{j}\right)=\frac{1}{y_{j i}}$, para todo $j$, e $\sigma_{i}(\vec{y})=\frac{1}{y_{i}}$. Pela hipótese,

$$
\lim _{j} y_{j i}=y_{i}
$$

Logo,

$$
\lim _{j} \frac{1}{y_{j i}}=\frac{1}{y_{i}}\left(=\sigma_{i}(\vec{y})\right) .
$$

Agora, de $(* * *)$, temos que $\left(\vec{y}_{j \sigma}\right)_{j} \in \pi_{n, m}[B]$. Portanto, $\vec{y}_{\sigma} \in \overline{\pi_{n, m}[B]}$.

Então, de (**), concluímos que $\vec{x} \in \overline{\pi_{n, m}[B]}=\bar{A}$.

Vejamos, agora, que $\bar{A} \subseteq \bigcup_{\sigma \in \Sigma} A_{\sigma}$.

Por conveniência, vamos dividir a demonstração deste caso em etapas.

Etapa 1: $A_{\sigma}$ é fechado, qualquer que seja $\sigma \in \Sigma$.

Com efeito, fixemos $\sigma \in \Sigma$. Seja $\vec{x} \in \bar{A}_{\sigma}$. Então, existe uma sequência $\left(\vec{x}_{j}\right)_{j}$ em $A_{\sigma}$ tal que $\vec{x}_{j} \rightarrow \vec{x}$.

Pela definição de $A_{\sigma}$ segue que

$$
\text { para todo } j \text { existe } \vec{y}_{j} \text { tal que } \vec{y}_{j} \in \pi_{n, m}\left[\overline{B_{\sigma}^{*}}\right]
$$

e

$$
y_{j i}=\left\{\begin{array}{cc}
x_{j i}, & \text { se } \sigma(i)=0 \\
\frac{1}{x_{j i}}, & \text { se } \sigma(i) \neq 0
\end{array}, \text { ou seja } \vec{y}_{j}=\vec{x}_{j \sigma}\right.
$$

Como $\vec{x}_{j} \rightarrow \vec{x}$, então $\left(\vec{y}_{j}=\right) \vec{x}_{j \sigma} \rightarrow \vec{x}_{\sigma}$.

Chamemos $\vec{x}_{\sigma}$ de $\vec{y}$. Então, $\vec{y} \in \pi_{n, m}\left[\overline{B_{\sigma}^{*}}\right]$.

De fato, de $\left(1^{*}\right)$, temos que

$$
\text { para todo } j \text { existe } \hat{y}_{j} \in \mathbb{R}^{n-m} \text { tal que }\left(\vec{y}_{j}, \hat{y}_{j}\right) \in \overline{B_{\sigma}^{*}} \text {. }
$$


Como $\overline{B_{\sigma}^{*}}$ é compacto, então $\left(\vec{y}_{j}, \hat{y}_{j}\right)_{j}$ possui subsequência convergente em $\overline{B_{\sigma}^{*}}$, digamos

$$
\left(\vec{y}_{j_{k}}, \hat{y}_{j_{k}}\right) \rightarrow(\vec{z}, \hat{z}) \in \overline{B_{\sigma}^{*}}
$$

Uma vez que $\vec{y}_{j} \rightarrow \vec{y}$, então $\lim _{k} y_{j_{k}}=\vec{y}$. Logo, $\vec{z}=\vec{y}$. Assim, $\vec{y} \in \pi_{n, m}\left[\overline{B_{\sigma}^{*}}\right]$.

Portanto, pela definição de $A_{\sigma}, \vec{x} \in A_{\sigma}$.

Etapa 2: $\bar{A} \subseteq \overline{\bigcup_{\sigma \in \Sigma} A_{\sigma}}$.

De fato, seja $\vec{x} \in \bar{A}$. Então, existe uma sequência $\left(\vec{x}_{j}\right)_{j}$ em $A$ tal que $\vec{x}_{j} \rightarrow \vec{x}$. Logo, para todo $j$, existe $\hat{x}_{j} \in \mathbb{R}^{n-m}$ tal que

$$
\bar{x}_{j}=\left(\vec{x}_{j}, \hat{x}_{j}\right) \in B
$$

Para cada $i \in\{1, \ldots, n\}$, temos $x_{j i} \in \mathbb{R}$. Assim,

$$
\underbrace{x_{j i} \leq-1}_{x_{j i} \in J_{-\infty}} \text { ou } \underbrace{-1 \leq x_{j i} \leq 1}_{x_{j i} \in J_{0}} \text { ou } \underbrace{x_{j i} \geq 1}_{x_{j i} \in J_{+\infty}} \text {. }
$$

De qualquer modo, existe $\sigma:\{1, \ldots, n\} \rightarrow\{-\infty, 0,+\infty\}$ tal que

$$
\bar{x}_{j} \in B_{\sigma}=B \cap\left(J_{\sigma_{1}} \times \cdots \times J_{\sigma(n)}\right) .
$$

Ou seja, para todo $j$, existe $\sigma \in \Sigma$ tal que $\bar{x}_{j} \in B_{\sigma}$.

Além disso,

$$
\bar{x}_{j \sigma} \in I_{\sigma},
$$

já que, para cada $i \in\{1, \ldots, n\}$,

se $\sigma(i)=0$, então $\sigma_{i}\left(\bar{x}_{j}\right)=x_{j i} \in J_{0}=I_{0}=I_{\sigma(i)}$; 
se $\sigma(i) \neq 0$, então $\sigma_{i}\left(\bar{x}_{j}\right)=\frac{1}{x_{j i}} \in\left\{\begin{array}{ll}I_{-\infty}, & \text { caso } x_{j i} \in J_{-\infty} \\ I_{+\infty}, & \text { caso } x_{j i} \in J_{+\infty}\end{array}\right.$.

Definamos, agora, $\bar{y}_{j}=\left(y_{j 1}, \ldots, y_{j n}\right)$ de sorte que

$$
y_{j i}=\left\{\begin{array}{cc}
x_{j i}, & \text { se } \sigma(i)=0 \\
\frac{1}{x_{j i}}, & \text { se } \sigma(i) \neq 0
\end{array} \text {, para } i \in\{1, \ldots, n\} \text { e todo } j \in \mathbb{N}\right.
$$

Então, $\bar{y}_{j \sigma}=\bar{x}_{j}$.

De $\left(2^{* *}\right)$, temos que $\bar{y}_{j} \in I_{\sigma}$.

De $\left(1^{* *}\right)$, temos que $\bar{y}_{j \sigma} \in B, i$. e.

$$
f\left(\bar{y}_{j \sigma}\right)=p\left(f_{1}\left(\bar{y}_{j \sigma}\right), \ldots, f_{l}\left(\bar{y}_{j \sigma}\right), \bar{y}_{j \sigma}\right)=0
$$

Logo, pela definição de $B_{\sigma}^{*}, \bar{y}_{j} \in B_{\sigma}^{*} \subseteq \overline{B_{\sigma}^{*}}$.

Portanto, $\vec{y}_{j} \in \pi_{n, m}\left[\overline{B_{\sigma}^{*}}\right]$. Segue, então, da definição de $A_{\sigma}$, que $\vec{x}_{j} \in A_{\sigma}$.

Em resumo, para todo $j$,

$$
\vec{x}_{j} \in \bigcup_{\sigma \in \Sigma} A_{\sigma}
$$

Portanto, $\vec{x} \in \overline{\bigcup_{\sigma \in \Sigma} A_{\sigma}}$.

Para concluir o caso em questão, observamos que $|\Sigma|<\omega$ e, assim,

$$
\overline{\bigcup_{\sigma \in \Sigma} A_{\sigma}}=\bigcup_{\sigma \in \Sigma} \bar{A}_{\sigma}
$$

Da Etapa 1, segue que

$$
\bigcup_{\sigma \in \Sigma} \bar{A}_{\sigma}=\bigcup_{\sigma \in \Sigma} A_{\sigma}
$$


Finalmente, da Etapa 2, concluímos que

$$
\bar{A} \subseteq \bigcup_{\sigma \in \Sigma} A_{\sigma}
$$

Seja $\exists \vec{y}_{\sigma} \phi_{\sigma}$ a fórmula que define $A_{\sigma}$, para cada $\sigma \in \Sigma$. Seja $p=|\Sigma|$. Então, $\bigcup_{\sigma \in \Sigma} A_{\sigma}$ é definido por

$$
\exists \vec{y}_{\sigma_{1}} \phi_{\sigma_{1}} \vee \cdots \vee \exists \vec{y}_{\sigma_{p}} \phi_{\sigma_{p}}
$$

que é equivalente, em $\langle\overline{\mathbb{R}},\{f: f \in R\}\rangle$, a

$$
\exists \vec{y}_{\sigma_{1}} \cdots \exists \vec{y}_{\sigma_{p}}\left(\phi_{\sigma_{1}} \vee \cdots \vee \phi_{\sigma_{p}}\right)
$$

Portanto, $\bar{A}$ é $\exists_{1}$-definível (em $\left.\langle\overline{\mathbb{R}},\{f: f \in R\}\rangle\right)$.

Notação. Para cada $f \in R$, seja $\tilde{f}: \mathbb{R}^{n} \rightarrow \mathbb{R}$ a função restrita dada por

$$
\tilde{f}(\bar{x})=\left\{\begin{array}{cl}
f(\bar{x}), & \text { se } \bar{x} \in[-1,1]^{n} \\
0, & \text { se } \bar{x} \in \mathbb{R}^{n} \backslash[-1,1]^{n}
\end{array} .\right.
$$

Escrevemos, também, $\tilde{R}_{n}=\left\{\tilde{f}: f \in R_{n}\right\}$ e $\tilde{R}=\bigcup_{n \in \mathbb{N}^{+}} \tilde{R}_{n}$.

O resultado seguinte (Teorema 3.1.3) é uma generalização de um resultado de Gabrielov em ([9], pág. 5), no qual ele assume que $\tilde{R}$ é uma coleção de funções analíticas restritas, que é fechado sob diferenciação parcial. Ele também assume que a coleção contém as funções coordenadas e as funções constantes e tal coleção forma um anel. No nosso caso, estas condições são omitidas.

Este resultado é mais forte do que precisa ser para nossos propósitos, que é obter (R4). Todavia, pode ser de algum interesse independente.

Antes de abordarmos, de fato, o Teorema 3.1.3, explicitamos abaixo uma proposição sobre a expansão de Taylor de funções do tipo $f: \mathbb{R}^{n} \rightarrow \mathbb{R}$. Tal asserção é afirmada aqui de modo 
idêntico à encontrada em ([13], pág. 360) e será usada no resultado subsequente.

Fato (Expansão de Taylor). Sejam $\kappa \in \mathbb{N}$ e $f: \mathbb{R}^{n} \rightarrow \mathbb{R}$ uma função que é $\mathcal{C}^{\kappa+1}$ numa vizinhança de uma bola fechada $\bar{B}(\bar{a}, r) \subseteq \mathbb{R}^{n}$. Então, para $\bar{x} \in B(\bar{a}, r)$,

$$
f(\bar{x})=\sum_{0 \leq j_{1}+\cdots+j_{n} \leq \kappa} \frac{\partial^{j_{1}+\cdots+j_{n}} f}{\partial x_{1}^{j_{1}} \cdots \partial x_{n}^{j_{n}}}(\bar{a}) \cdot \frac{\left(x_{1}-a_{1}\right)^{j_{1}} \cdots\left(x_{n}-a_{n}\right)^{j_{n}}}{\left(j_{1}\right) ! \cdots\left(j_{n}\right) !}+\mathcal{R}_{\kappa, \bar{a}}(\bar{x})
$$

sendo que

$$
\left|\mathcal{R}_{\kappa, \bar{a}}(\bar{x})\right| \leq C_{0} \frac{\|\bar{x}-\bar{a}\|^{\kappa+1}}{(\kappa+1) !}
$$

$e$

$$
C_{0}=\sup _{\substack{\bar{y} \in \bar{B}(\bar{a}, r) \\ j_{1}+\cdots+j_{n}=\kappa+1}}\left|\frac{\partial^{j_{1}+\cdots+j_{n}} f}{\partial x_{1}^{j_{1}} \cdots \partial x_{n}^{j_{n}}}(\bar{y})\right|
$$

Teorema 3.1.3. Suponha que $R$ satisfaça (R2) e (R6). Seja $X \subseteq[-1,1]^{n}$ um conjunto da forma

$$
X=\left\{\bar{x} \in[-1,1]^{n}: \tilde{f}(\bar{x})=0 \wedge \tilde{g}_{1}(\bar{x})>0 \wedge \cdots \wedge \tilde{g}_{k}(\bar{x})>0\right\}
$$

de sorte que

$$
\tilde{f} \text { é da forma } p\left(\bar{x}, \tilde{f}_{1}(\bar{x}), \ldots, \tilde{f}_{l}(\bar{x})\right) \text {, }
$$

$\operatorname{com} p \in \mathbb{R}\left[z_{1}, \ldots, z_{n+l}\right]$ e $\tilde{f}_{1}, \ldots, \tilde{f}_{l} \in \tilde{R}$, e cada $\tilde{g}_{i}$ ou é um polinômio ou está em $\tilde{R}$. Então,

$$
\bar{X} \text { é definivel sem quantificadores em }\langle\overline{\mathbb{R}},\{\tilde{f}: \tilde{f} \in \tilde{R}\}\rangle \text {. }
$$

Demonstração. Introduziremos alguma notação que nos será bastante útil.

\section{Notação.}

- Sempre que uma propriedade for verdadeira para todos os $\tilde{g}_{i}$ 's, diremos que é verdadeira para $\tilde{g}$. Por exemplo, $X=\{\bar{x}: \tilde{f}(\bar{x})=0 \wedge \tilde{g}(\bar{x})>0\}$.

- A função $\|\cdot\|: \mathbb{R}^{n} \rightarrow \mathbb{R}$ denota a norma euclideana. 
Para facilitar a demonstração, vamos proceder através de etapas.

Etapa A:

Seja

$$
A=\left\{(\varepsilon, \delta): \exists \bar{x}\left[d(\bar{x}, X)<\frac{\varepsilon}{2} \wedge \sup \left\{\tilde{g}_{\min }(\bar{y}): \bar{y} \in B[\bar{x}, \varepsilon]\right\}=\delta\right\}\right.
$$

em que

$$
\begin{aligned}
\tilde{g}_{\min }(\bar{y}) & =\min \left\{\tilde{g}_{1}(\bar{y}), \ldots, \tilde{g}_{k}(\bar{y})\right\} \\
B[\bar{x}, \varepsilon] & =\{\bar{y} \in X:\|\bar{y}-\bar{x}\| \leq \varepsilon\}
\end{aligned}
$$

e $d$ é a função distância.

Note que $A \subseteq] 0, \infty[\times] 0, \infty[$.

Vejamos que existem $\kappa \in \mathbb{N}^{+}$e $\rho_{1}>0$, tal que

$$
\text { se }(\varepsilon, \delta) \in A \text { e } \varepsilon \in] 0, \rho_{1}\left[\text {, então } \varepsilon^{\kappa-1}<\delta\right. \text {. }
$$

AfirmaÇÃo 1. Seja $(\varepsilon, \delta) \in \bar{A}$. Se $\delta=0$, então $\varepsilon=0$.

Prova da Afirmação. Suponha, por contradição, que $\varepsilon>0$.

De $(\varepsilon, 0) \in \bar{A}$, segue que existe uma sequência $\left(\varepsilon_{i}, \delta_{i}\right)_{i}$ em $A$ tal que $\varepsilon_{i} \rightarrow \varepsilon$ e $\delta_{i} \rightarrow 0$. Pela definição de $A$, existe uma sequência $\left(\bar{z}_{i}\right)_{i}$ tal que

$$
d\left(\bar{z}_{i}, X\right)<\frac{\varepsilon_{i}}{2} \quad \text { e } \quad \sup _{\bar{y} \in B\left[\bar{z}_{i}, \varepsilon_{i}\right]} \tilde{g}_{\min }(\bar{y})=\delta_{i} .
$$

Logo, $\left(\bar{z}_{i}\right)_{i}$ é limitada ${ }^{1}$.

Por Bolzano-Weierstrass, $\left(\bar{z}_{i}\right)_{i}$ tem subsequência convergente. Por conveniência, vamos escrever a subsequência como $\left(\bar{z}_{i}\right)_{i}$ ao invés de $\left(\bar{z}_{i_{j}}\right)_{j}$.

\footnotetext{
${ }^{1}$ Primeiro, $X \subseteq[-1,1]^{n} \subseteq B(0, \sqrt{n})$ (bola aberta de centro 0 e raio $\sqrt{n}$ ). Além disso, $\left(\varepsilon_{i}\right)_{i}$ é limitada, pois é convergente. Consequentemente, existe $s \in \mathbb{R}^{+}$tal que $\left.\varepsilon_{i} \in\right]-s, s[$, para todo $i \in \mathbb{N}$. Portanto, $\bar{z}_{i} \in B(0, s+\sqrt{n})$, para qualquer $i \in \mathbb{N}^{+}$.
} 
Seja $\bar{z}=\lim _{i} \bar{z}_{i}$. Então,

$$
\lim _{i} d\left(\bar{z}_{i}, X\right)=d(\bar{z}, X)
$$

Assim, tomando o limite de $d\left(\bar{z}_{i}, X\right)<\frac{\varepsilon_{i}}{2}$, obtemos

$$
d(\bar{z}, X) \leq \frac{\varepsilon}{2}
$$

Uma vez que

$$
d(\bar{z}, X)=\inf \{\|\bar{w}-\bar{z}\|: \bar{w} \in X\}
$$

então existe $\bar{y}_{0} \in X$ de sorte que

$$
\left\|\bar{y}_{0}-\bar{z}\right\| \leq \frac{\varepsilon}{2} .
$$

Vejamos, agora, que para todo $\bar{w} \in X$ tal que $\|\bar{w}-\bar{x}\| \leq \frac{\varepsilon}{2}$, segue que $\tilde{g}_{\min }(\bar{w}) \leq 0$.

Com efeito, fixemos $\bar{y} \in X$, tal que $\|\bar{y}-\bar{x}\| \leq \frac{\varepsilon}{2}$, e seja $r>0$ arbitrário.

De $\delta_{i} \rightarrow 0$, segue que existe $J_{0} \in \mathbb{N}$, de sorte que $\delta_{i}<r$, para todo $i>J_{0}$.

De $\bar{z}_{i} \rightarrow \bar{z}$, segue que existe $J_{1} \in \mathbb{N}$ de modo que $\left\|\bar{z}_{i}-\bar{z}\right\|<\frac{\varepsilon}{8}$, para qualquer $i>J_{1}$.

Logo,

$$
\left\|\bar{z}_{i}-\bar{y}\right\| \leq\left\|\bar{z}_{i}-\bar{z}\right\|+\|\bar{y}-\bar{z}\|<\frac{\varepsilon}{8}+\frac{\varepsilon}{2}=\frac{5 \varepsilon}{8}, \text { para } i>J_{1}
$$

De $\varepsilon_{i} \rightarrow \varepsilon$, temos que existe $J_{2} \in \mathbb{N}$ tal que $\varepsilon_{i}>\frac{5 \varepsilon}{8}$, qualquer que seja $i>J_{2}$.

Agora, tome $J_{3}>\max \left\{J_{0}, J_{1}, J_{2}\right\}$.

Como $J_{3}>J_{1}$, então $\left\|\bar{y}-\bar{z}_{J_{3}}\right\| \leq \frac{5 \varepsilon}{8}$. Logo,

$$
\tilde{g}_{m i n}(\bar{y}) \leq \sup \left\{\tilde{g}_{m i n}(\bar{w}):\left\|\bar{w}-\bar{z}_{J_{3}}\right\| \leq \frac{5 \varepsilon}{8}\right\}
$$

Já que $\varepsilon_{J_{3}}>\frac{5 \varepsilon}{8}$, pois $J_{3}>J_{2}$, então

$$
\sup \left\{\tilde{g}_{\min }(\bar{w}):\left\|\bar{w}-\bar{z}_{J_{3}}\right\| \leq \frac{5 \varepsilon}{8}\right\} \leq \sup \left\{\tilde{g}_{\min }(\bar{w}):\left\|\bar{w}-\bar{z}_{J_{3}}\right\| \leq \varepsilon_{J_{3}}\right\}=\delta_{J_{3}} .
$$


Finalmente, dado que $J_{3}>J_{0}$, temos $\delta_{J_{3}}<r$. Portanto, $\tilde{g}_{\min }(\bar{y})<r$.

Assim, $\tilde{g}_{\min }(\bar{y}) \leq 0$.

Logo, pela definição de $X, \bar{y} \notin X .(\Rightarrow \Leftarrow)$

Portanto, $\varepsilon=0$.

Seja $\theta:] 0, \infty[\rightarrow \mathbb{R}$ dada por

$$
\theta(x)=\inf A_{x}
$$

Como $A$ é definível em $\langle\overline{\mathbb{R}},\{\tilde{f}: \tilde{f} \in \tilde{R}\}\rangle$, então $\theta$ também o é.

Uma vez que, para todo $x \in] 0, \infty[$, temos $(x, \theta(x)) \in \bar{A}$. Assim, pela Afirmação $1, \theta(x)>0$. Dessa forma, podemos definir $\Psi:] 0, \infty[\rightarrow] 0, \infty[$ por

$$
\Psi(x)=\frac{1}{\theta\left(\frac{1}{x}\right)} .
$$

A função $\Psi$ é definível em $\langle\overline{\mathbb{R}},\{\tilde{f}: \tilde{f} \in \tilde{R}\}\rangle$, já que $\theta$ o é. Portanto, por (R6b), existem $a \in \mathbb{R}^{+}$e $p \in \mathbb{N}$ tal que

$$
\Psi(x)<x^{p} \text {, para } x>a .
$$

Consequentemente,

$$
\theta\left(\frac{1}{x}\right)>\left(\frac{1}{x}\right)^{p}
$$

Tome $\rho_{1}=\frac{1}{a}$ e $\kappa=p+1$. Então, escrevendo $\varepsilon=\frac{1}{x}$, segue que

$$
\left.\theta(\varepsilon)>\varepsilon^{\kappa-1} \text {, para qualquer } \varepsilon \in\right] 0, \rho_{1}[\text {. }
$$

Para $\varepsilon \in] 0, \rho_{1}[$ arbitrário, temos, pela definição de $\theta$, que

$$
\theta(\varepsilon) \leq \delta \text {, qualquer que seja } \delta \in A_{\varepsilon}
$$

Assim, segue $\left(1^{*}\right)$. 
Aviso. Fixemos $\kappa$ e $\rho_{1}$ como na Etapa A, daqui até o fim da demonstração.

Etapa B:

Seja $\psi:[0,+\infty[\rightarrow \mathbb{R}$ com a propriedade:

para todo $C>0$ existe $a>0$ tal que $|\psi(t)| \leq C\left|t^{\kappa}\right|$, sempre que $\left.t \in\right] 0, a[$.

Vejamos, então, que existe $\rho>0$ tal que

$$
\text { se }(\varepsilon, \delta) \in A \text { e } \varepsilon \in] 0, \rho\left[\text {, então } \varepsilon^{\kappa}+\psi(\varepsilon)<\delta\right. \text {. }
$$

De fato, para $C=1$, temos que existe $\rho_{2}>0$ de modo que

$$
\left.|\psi(t)| \leq\left|t^{\kappa}\right|=t^{\kappa} \text {, para todo } t \in\right] 0, \rho_{2}[
$$

Tome $\rho_{3}=\min \left\{\rho_{1}, \rho_{2}, \frac{1}{2}\right\}$.

Logo, para $\varepsilon \in] 0, \rho_{3}[$ temos

$$
\varepsilon^{\kappa}+\psi(\varepsilon) \leq \varepsilon^{\kappa}+\varepsilon^{\kappa}=2 \varepsilon^{\kappa-1} \varepsilon \leq 2 \varepsilon^{\kappa-1} \frac{1}{2}=\varepsilon^{\kappa-1} \stackrel{\left(1^{*}\right)}{<} \delta
$$

\section{Etapa $C$ :}

Seja $\tilde{g}_{\kappa, \bar{x}, i}$ a soma dos termos que têm ordem no máximo $\kappa$ e aparecem na expansão de Taylor de $\tilde{g}_{i}$ em torno do ponto $\bar{x}$.

Novamente, sempre que uma propriedade é válida para todos os $\tilde{g}_{\kappa, \bar{x}, i}$ 's, escrevemos que é válida para $\tilde{g}_{\kappa, \bar{x}}$. 
Definimos $\phi(\bar{x}, \bar{y})$ pela seguinte fórmula

$$
\left(\tilde{g}_{\kappa, \bar{x}}(\bar{y}) \geq\|\bar{x}-\bar{y}\|^{\kappa}\right) \wedge(\tilde{g}(\bar{x})>0 \vee\|\bar{x}-\bar{y}\|>0)
$$

O importante desta definição é que a variável $\bar{y}$ ocorre livre na fórmula $\phi(\bar{x}, \bar{y})$, fato que usaremos na Etapa G.

Agora, considere

$$
G_{\bar{x}}=\{\bar{y}: \tilde{f}(\bar{y})=0 \wedge \phi(\bar{x}, \bar{y})\} .
$$

AfIRMAÇÃo 2. $\bar{a} \in X$ se, e somente se, $\bar{a} \in G_{\bar{a}}$.

Prova da Afirmação. Pela definição de $X$, segue que $\bar{a} \in X$ se, e somente se, $\tilde{f}(\bar{a})=0$ e $\tilde{g}(\bar{a})>0$.

Além disso, $\tilde{g}_{\kappa, \bar{a}}(\bar{a})=0$. Logo, $\phi(\bar{a}, \bar{a})$ vale e, assim, $\bar{a} \in G_{\bar{a}}$.

Por outro lado, de $\bar{a} \in G_{\bar{a}}$, temos que $\tilde{f}(\bar{a})=0, \tilde{g}_{\kappa, \bar{a}}(\bar{a})=0$ e $\tilde{g}(\bar{a})>0$. Portanto, $\bar{a} \in X$.

Agora, seja $\psi:[0,+\infty[\rightarrow \mathbb{R}$ a função dada por

$$
\psi_{g}(t)=\sup \left\{\left|\tilde{g}_{\kappa, \bar{a}, i}(\bar{y})-\tilde{g}_{i}(\bar{y})\right|:\|\bar{y}-\bar{a}\| \leq t, i=1, \ldots, k\right\}
$$

Por (R6a), as funções $\tilde{g}_{i}{ }^{\prime}$ s são $\mathcal{C}^{\infty}$. Em particular, são $\mathcal{C}^{\kappa+1}$.

Afirmamos que $\lim _{t \rightarrow 0} \frac{\psi_{g}(t)}{t^{\kappa}}=0$.

Com efeito, seja $s_{i}>0$ tal que $\bar{B}\left(\bar{a}, s_{i}\right) \subseteq[-1,1]^{n}$, para cada $i \in\{1, \ldots, k\}$.

Então, para todo $\bar{y} \in B\left(\bar{a}, s_{i}\right)$,

$\tilde{g}(\bar{y})=\sum_{0 \leq j_{1}+\cdots+j_{n} \leq \kappa} \frac{\partial^{j_{1}+\cdots+j_{n}} \tilde{g}_{i}}{\partial x^{j_{1}} \cdots \partial x^{j_{n}}}(\bar{a}) \cdot \frac{\left(y_{1}-a_{1}\right)^{j_{1}} \cdots\left(y_{n}-a_{n}\right)^{j_{n}}}{j_{1} ! \cdots j_{n} !}+\mathcal{R}_{\kappa, \bar{a}, i}(\bar{y})=\tilde{g}_{\kappa, \bar{a}, i}(\bar{y})+\mathcal{R}_{\kappa, \bar{a}, i}(\bar{y})$,

o que implica

$$
\left|\tilde{g}_{\kappa, \bar{a}, i}(\bar{y})-\tilde{g}_{i}(\bar{y})\right|=\left|\mathcal{R}_{\kappa, \bar{a}, i}(\bar{y})\right|,
$$


com

$$
\left|\mathcal{R}_{\kappa, \bar{a}, i}(\bar{y})\right| \leq \frac{C_{0 i}}{(\kappa+1) !}\|\bar{y}-\bar{a}\|^{\kappa+1}
$$

Seja $C_{0}=\max \left\{C_{01}, \ldots, C_{0 k}\right\}$. Então,

$$
\left|\mathcal{R}_{\kappa, \bar{a}, i}(\bar{y})\right| \leq \frac{C_{0}}{(\kappa+1) !}\|\bar{y}-\bar{a}\|^{\kappa+1}
$$

Seja, ainda, $s=\frac{1}{2} \min \left\{s_{1}, \ldots, s_{k}\right\}$. Então, para qualquer $\bar{y}$ tal que $\|\bar{y}-\bar{a}\| \leq s$,

$$
\left|\mathcal{R}_{\kappa, \bar{a}, i}(\bar{y})\right| \leq \frac{C_{0}}{(\kappa+1) !}\|\bar{y}-\bar{a}\|^{\kappa+1}, \text { para } i=1, \ldots, k .
$$

Fixe $t \in] 0, s]$. Assim, dado $\bar{y}$, de sorte que $\|\bar{y}-\bar{a}\| \leq t$,

$$
\left|\mathcal{R}_{\kappa, \bar{a}, i}(\bar{y})\right| \leq \frac{C_{0}}{(k+1) !}\|\bar{y}-\bar{a}\|^{\kappa+1} \leq \frac{C_{0}}{(\kappa+1) !} t^{\kappa+1}
$$

Em resumo, para todo $t \in] 0, s], \bar{y} \in\{\bar{w}:\|\bar{w}-\bar{a}\| \leq t\}$ e todo $i \in\{1, \ldots, k\}$,

$$
\left|\mathcal{R}_{\kappa, \bar{a}, i}(\bar{y})\right| \leq \frac{C_{0}}{(\kappa+1) !} t^{\kappa+1}
$$

Logo,

$$
\psi_{g}(t)=\sup _{\substack{\|\bar{y}-\bar{a}\| \leq t \\ i=1, \ldots, k}}\left|\mathcal{R}_{\kappa, \bar{a}, i}(\bar{y})\right| \leq \frac{C_{0}}{(\kappa+1) !} t^{\kappa+1}
$$

Portanto, segue que $\lim _{t \rightarrow 0} \frac{\psi_{g}(t)}{t^{\kappa}}=0$.

Como consequência imediata da definição de limite, existe $\rho_{3}>0$ tal que

$$
\left.(\star) \quad\left|\psi_{g}(t)\right|<t^{\kappa} \text {, para todo } t \in\right] 0, \rho_{3}[.
$$

AfirmaÇÃo 3. Se $\bar{a} \in \bar{G}_{\bar{a}}$, então $\bar{a} \in \bar{X}$. 
Prova da Afirmação. Sejam $\bar{a} \in \bar{G}_{\bar{a}}$ e $\gamma>0$. Então, existe $\bar{c} \in X$ tal que $\|\bar{a}-\bar{c}\|<\gamma$.

Com efeito, seja $\bar{c} \in G_{\bar{a}}$ tal que $\|\bar{a}-\bar{c}\|<\min \left\{\gamma, \rho_{3}\right\}^{2}$, sendo que $\rho_{3}$ é o real positivo, mencionado imediatamente antes do enunciado da Afirmação 3 , que goza da propriedade $(\star)$. Então,

$$
\tilde{g}_{\kappa, \bar{a}}(\bar{c}) \geq\|\bar{a}-\bar{c}\|^{\kappa} .
$$

Como $\|\bar{a}-\bar{c}\| \in] 0, \rho_{3}[, \log O$

$$
\left|\psi_{g}(\|\bar{a}-\bar{c}\|)\right|<\|\bar{a}-\bar{c}\|^{\kappa} .
$$

Pela definição de $\psi_{g}$,

$$
\left|\tilde{g}_{\kappa, \bar{a}}(\bar{c})-\tilde{g}(\bar{c})\right| \leq \psi_{g}(\|\bar{a}-\bar{c}\|) .
$$

Combinando estas desigualdades, chegamos a

$$
\left|\tilde{g}_{\kappa, \bar{a}}(\bar{c})-\tilde{g}(\bar{c})\right|<\tilde{g}_{\kappa, \bar{a}}(\bar{c}) .
$$

Portanto, $\tilde{g}(\bar{c})>0$.

Visto que $\tilde{f}(\bar{c})=0$ (pois, $\bar{c} \in C_{\bar{a}}$ ), então $\bar{c} \in X$.

Assim, para todo $j>0$ existe $\bar{c}_{j} \in X$ tal que $\left\|\bar{c}_{j}-\bar{a}\right\|<\frac{1}{j}$.

Resta mostrar que $\lim _{j} \bar{c}_{j}=\bar{a}$.

De fato, dado $r>0$ qualquer, temos que existe $N_{0} \in \mathbb{N}$ tal que $\frac{1}{N_{0}}<r$. Logo, para todo $j>N_{0}$, temos

$$
\left\|\bar{c}_{j}-\bar{a}\right\|<\frac{1}{j}<\frac{1}{N_{0}}<r .
$$

Ou seja, $\bar{a} \in \bar{X}$.

\footnotetext{
${ }^{2}$ De $\bar{a} \in \bar{G}_{\bar{a}}$, temos que existe uma sequência $\left(\bar{c}_{j}\right)_{j}$ em $G_{\bar{a}}$ tal que $\bar{c}_{j} \rightarrow \bar{a}$. Pela definição de convergência, para $\min \left\{\gamma, \rho_{3}\right\}$ existe $J \in \mathbb{N}$ de sorte que, para todo $j>J,\left\|\overline{c_{j}}-\bar{a}\right\|<\min \left\{\gamma, \rho_{3}\right\}$. Basta tomar $\bar{c}=\bar{c}_{j}$, para algum $j>J$.
} 
AfirmaÇÃo 4. Se $\bar{a} \in \bar{X} \backslash X$, então $\bar{a} \in \bar{G}_{\bar{a}}$.

Prova da Afirmação. Sejam $\bar{a} \in \bar{X} \backslash X$ e $\gamma>0$. Então, existe $\bar{c} \in \bar{G}_{\bar{a}}$ tal que $\|\bar{a}-\bar{c}\|<\gamma$.

De fato, analogamente à Afirmação 3 , seja $\bar{b} \in X$ tal que $\|\bar{b}-\bar{a}\|<\min \{\gamma, \rho\}$, de modo que $\rho$ seja como na propriedade $\left(2^{*}\right)$, na Etapa B, para a função $\psi_{g}$.

Chamemos $\varepsilon=\|\bar{b}-\bar{a}\|$.

Seja, também,

$$
\delta=\sup _{\substack{\bar{y} \in X \\\|\bar{y}-\bar{a}\| \leq \varepsilon}} \tilde{g}_{\min }(\bar{y})
$$

Então $^{3}, d(\bar{a}, X)<\frac{\varepsilon}{2}$. Portanto, pela definição de $A,(\varepsilon, \delta) \in A$. Uma vez que $\left.\varepsilon \in\right] 0, \rho[$, segue, de $\left(2^{*}\right)$, que

$$
\varepsilon^{\kappa}+\psi_{g}(\varepsilon)<\delta
$$

Logo, da definição de supremo, existe $\bar{c} \in X$, com $\|\bar{a}-\bar{c}\| \leq \varepsilon$, tal que

$$
\varepsilon^{\kappa}+\psi_{g}(\varepsilon)<\tilde{g}_{\min }(\bar{c})
$$

ou seja

$$
\varepsilon^{\kappa}<\tilde{g}_{\min }(\bar{c})-\psi_{g}(\varepsilon) .
$$

Por definição de $\psi_{g}$,

$$
\psi_{g}(\varepsilon) \geq\left|\tilde{g}_{\kappa, \bar{a}}(\bar{c})-\tilde{g}(\bar{c})\right| .
$$

Em particular,

$$
\tilde{g}_{\kappa, \bar{a}}(\bar{c})-\tilde{g}(\bar{c}) \geq-\psi_{g}(\bar{\varepsilon}),
$$

ou seja

$$
\tilde{g}_{\kappa, \bar{a}}(\bar{c}) \geq \tilde{g}(\bar{c})-\psi_{g}(\bar{\varepsilon}) .
$$

\footnotetext{
${ }^{3}$ De $\bar{a} \in \bar{X} \backslash X$ temos que existe uma sequência $\left(\bar{c}_{j}\right)_{j}$ em $X$ tal que $\bar{c}_{j} \rightarrow \bar{a}$. Logo, para $\frac{\varepsilon}{2}$, existe $J \in \mathbb{N}$ tal que $\forall j>J,\left\|\bar{a}-\bar{c}_{j}\right\|<\frac{\varepsilon}{2}$. Portanto, pela definição de distância de um ponto a um conjunto, $d(\bar{a}, X)<\frac{\varepsilon}{2}$.
} 
Portanto,

$$
\tilde{g}_{\kappa, \bar{a}}(\bar{c})>\varepsilon^{\kappa} \geq\|\bar{a}-\bar{c}\|^{\kappa}
$$

Uma vez que $\tilde{f}(\bar{c})=0$ e $\tilde{g}(\bar{c})>0$ (pois, $\bar{c} \in X), \bar{c} \in G_{\bar{a}}$.

Analogamente à Afirmação 3 , temos, portanto, que existe uma sequência $\left(\bar{c}_{j}\right)_{j}$ em $G_{\bar{a}}$ de sorte que $\lim _{j} \bar{c}_{j}=\bar{a}$. Assim, $\bar{a} \in \bar{G}_{\bar{a}}$.

Das afirmações 2, 3 e 4, segue, imediatamente, que $\bar{a} \in \bar{X}$ se, e somente se, $\bar{a} \in \bar{G}_{\bar{a}}$.

Etapa D:

Considere

$$
\begin{aligned}
B=\{(\varepsilon, \delta): \varepsilon>0 & \wedge \exists \bar{x} \in[-1,1]^{n}(d(\bar{x}, X) \geq \varepsilon \\
& \left.\left.\wedge \sup _{\beta \in] 0, \varepsilon]} \min _{\bar{y} \in \mathbb{R}^{n}}\{|\tilde{f}(\bar{y})|: \phi(\bar{x}, \bar{y}) \wedge\|\bar{x}-\bar{y}\|=\beta\}=\delta\right)\right\} .
\end{aligned}
$$

Note que $B \subseteq] 0, \infty[\times] 0, \infty[$.

Também, se $\varepsilon$ é tal que existe $\delta$, com $(\varepsilon, \delta) \in B$, então

$$
\{|\tilde{f}(\bar{y})|: \phi(\bar{x}, \bar{y}) \wedge\|\bar{x}-\bar{y}\|=\beta\} \neq \emptyset, \text { para todo } \beta \in] 0, \varepsilon]
$$

Vejamos que existem $\lambda \in \mathbb{N}^{+}$e $\eta_{1}>0$ tais que

$$
\text { se }(\varepsilon, \delta) \in B \text { e } \varepsilon \in] 0, \eta_{1}\left[\text {, então } \varepsilon^{\lambda-1}<\delta\right. \text {. }
$$

AfirmaÇÃo 5. Seja $(\varepsilon, \delta) \in \bar{B}$. Se $\delta=0$, então $\varepsilon=0$.

Prova da Afirmação. Suponha, por absurdo, que $\varepsilon>0$.

De $(\varepsilon, 0) \in \bar{B}$, temos que existe uma sequência $\left(\varepsilon_{j}, \delta_{j}\right)_{j}$ em $B$ tal que $\varepsilon_{j} \rightarrow \varepsilon$ e $\delta_{j} \rightarrow 0$. 
Logo, pela definição de $B$, existe uma sequência $\left(\bar{z}_{j}\right)_{j}$ em $[-1,1]^{n}$ de sorte que

$$
d\left(\bar{z}_{j}, X\right) \geq \varepsilon_{j} \quad \text { e } \quad \sup _{\left.\beta \in] 0, \varepsilon_{j}\right]} \min _{\bar{y} \in \mathbb{R}^{n}}\left\{|\tilde{f}(\bar{y})|: \phi\left(\bar{z}_{j}, \bar{y}\right) \wedge\left\|\bar{z}_{j}-\bar{y}\right\|=\beta\right\}=\delta_{j} .
$$

Como $\left(\bar{z}_{j}\right)_{j}$ é limitada (já que, $\bar{z}_{j} \in[-1,1]^{n}$, para todo $j$ ), então possui subsequência convergente. Por conveniência, chamemos tal subsequência de $\left(\bar{z}_{j}\right)_{j}$.

Seja $\lim _{j} \bar{z}_{j}=\bar{z}$.

Uma vez que $d\left(\bar{z}_{j}, X\right) \geq \varepsilon_{j}$, então $d(\bar{z}, X) \geq \varepsilon$.

Tome $\beta \in] 0, \varepsilon[$. Seja $J \in \mathbb{N}$ tal que $\beta \in] 0, \varepsilon_{j}\left[\right.$, para todo $j \geq J^{4}$. Como

$$
\left\{|\tilde{f}(\bar{y})|: \phi(\bar{z}, \bar{y}) \wedge\left\|\bar{z}_{j}-\bar{y}\right\|=\beta\right\} \neq \emptyset, \text { para todo } j \geq J
$$

então existe uma sequência de testemunhas $\left(\bar{u}_{j}\right)_{j \geq J}$ em $\mathbb{R}^{n}$ tal que, para $j \geq J$ :

(i) $\left\|\bar{z}_{j}-\bar{u}_{j}\right\|=\beta$;

(ii) $\phi\left(\bar{z}_{j}, \bar{u}_{j}\right)$ vale;

(iii) $\left|\tilde{f}\left(\bar{u}_{j}\right)\right| \leq \delta_{j}$

De $(i)$, segue que $\left(\bar{u}_{j}\right)_{j}$ é limitada ${ }^{5}$. Portanto, possui subsequência convergente. Por conveniência, escrevemos tal subsequência como $\left(\bar{u}_{j}\right)_{j \geq J}$.

Seja $\bar{u}=\lim _{j} \bar{u}_{j}$.

Uma vez que $\tilde{f}$ é contínua, $\tilde{f}\left(\bar{u}_{j}\right) \rightarrow f(\bar{u})$. Além disso, temos, de $(i i i)$, que $\left|\tilde{f}\left(\bar{u}_{j}\right)\right| \leq \delta_{j}$, para todo $j \geq J$. Portanto,

$$
|\tilde{f}(\bar{u})|=\left|\lim \tilde{f}\left(\bar{u}_{j}\right)\right| \leq \lim \delta_{j}=0, \text { ou seja }|\tilde{f}(\bar{u})|=0 .
$$

\footnotetext{
${ }^{4}$ Seja $r=\varepsilon-\beta>0$. Então, de $\varepsilon_{j} \rightarrow \varepsilon$, segue que existe $J^{\prime} \in \mathbb{N}$ tal que $\beta=\varepsilon-r<\varepsilon_{j}<\varepsilon+r$, para todo $j>J^{\prime}$. Basta, portanto, tomarmos $J=J^{\prime}+1$.

${ }^{5}$ Para todo $j \geq J,\left\|\bar{u}_{j}\right\| \leq\left\|\bar{u}_{j}-\bar{z}_{j}\right\|+\left\|\bar{z}_{j}\right\| \leq \beta+\sqrt{n}$. Pois, $\left\|\bar{z}_{j}-\bar{u}_{j}\right\|=\beta$ e $\left\|\bar{z}_{j}\right\| \in[-1,1]^{n} \subseteq B(0, \sqrt{n})$.
} 
Logo,

$$
\tilde{f}(\bar{u})=0 .
$$

De $(i)$, segue que $\left\|\bar{z}_{j}-\bar{u}_{j}\right\|=\beta$, para todo $j \geq J$. Assim,

$$
\|\bar{z}-\bar{u}\|=\beta
$$

De $(i i)$, temos que $\tilde{g}_{\kappa, \bar{z}_{j}}\left(\bar{u}_{j}\right) \geq\left\|\bar{z}_{j}-\bar{u}_{j}\right\|^{\kappa}$, para todo $j \geq J$. Então,

$$
\tilde{g}_{\kappa, \bar{z}}(\bar{u}) \geq\|\bar{z}-\bar{u}\|^{\kappa}
$$

Assim,

$$
\tilde{g}(\bar{u}) \geq\|\bar{z}-\bar{u}\|^{\kappa}=\beta^{\kappa}>0 .
$$

Logo, $\bar{u} \in X$.

Mas, $d(\bar{z}, X) \geq \varepsilon>\beta=\|\bar{z}-\bar{u}\|$. Contradição.

Portanto, $\varepsilon=0$.

Identicamente à Etapa $\mathrm{A}$, segue, da Afirmação 5 e de (R6b), que existem $\eta_{1}>0$ e $\lambda \in \mathbb{N}^{+}$ tal que $\left(3^{*}\right)$ é válida.

Etapa E:

Seja $\psi:[0, \infty[\rightarrow \mathbb{R}$ uma função tal que

$$
\lim _{t \rightarrow 0} \frac{\psi(t)}{t^{\lambda}}=0
$$

Então, existe $\eta>0$ tal que

$$
\text { se }(\varepsilon, \delta) \in B \text { e } \varepsilon \in] 0, \eta\left[\text {, então } \varepsilon^{\lambda}+\psi(\varepsilon)<\delta\right. \text {. }
$$


Com efeito, de $\lim _{t \rightarrow 0} \frac{\psi(t)}{t^{\lambda}}=0$, temos que existe $\eta_{2}>0$ tal que

$$
|\psi(\varepsilon)|<\varepsilon^{\lambda}
$$

sempre que $\varepsilon \in] 0, \eta_{2}[$.

Tome $\eta=\min \left\{\eta_{1}, \eta_{2}, \frac{1}{2}\right\}$.

Então, para todo $\varepsilon \in] 0, \eta_{2}[$,

$$
\varepsilon^{\lambda}+\psi(\varepsilon)<\varepsilon^{\lambda}+\varepsilon^{\lambda}=2 \varepsilon^{\lambda-1} \varepsilon \leq 2 \varepsilon^{\lambda-1} \frac{1}{2}=\varepsilon^{\lambda-1} \stackrel{\left(4^{*}\right)}{<} \delta .
$$

Etapa $F$ :

Denotemos por $\tilde{f}_{\lambda, \bar{x}}$ a soma dos termos que têm ordem, no máximo, $\lambda$ e aparecem na expansão de Taylor de $\tilde{f}$ em torno de $\bar{x}$.

Considere

$$
F_{\bar{x}}=\left\{\bar{y}: \phi(\bar{x}, \bar{y}) \wedge\left|\tilde{f}_{\lambda, \bar{x}}(\bar{y})\right| \leq\|\bar{x}-\bar{y}\|^{\lambda}\right\}
$$

Então, $\bar{a} \in \bar{X}$ se, e somente se, $\bar{a} \in \bar{F}_{\bar{a}}$.

Com efeito, seja $\bar{a} \in \bar{X}$. Seja $r_{1}>0$ arbitrário. Vamos mostrar que existe $\bar{b} \in F_{\bar{a}}$ tal que $\|\bar{a}-\bar{b}\|<r_{1}$.

Por (R6a), $\tilde{f}$ é, em particular, de classe $\mathcal{C}^{\lambda+1}$. Logo, para $\left.\bar{y} \in\right]-1,1\left[^{n}\right.$,

$$
\tilde{f}(\bar{y})=\tilde{f}_{\lambda, \bar{a}}(\bar{y})+\mathcal{R}_{\lambda, \bar{a}}(\bar{y})
$$

com

$$
\left|\mathcal{R}_{\lambda, \bar{a}}(\bar{y})\right| \leq C_{0} \frac{\|\bar{y}-\bar{a}\|^{\lambda+1}}{(\lambda+1) !} \quad \text { e } \quad C_{0}>0
$$

Assim,

$$
\left|\tilde{f}_{\lambda, \bar{a}}(\bar{y})\right| \leq|\tilde{f}(\bar{y})|+\left|\mathcal{R}_{\lambda, \bar{a}}(\bar{y})\right|
$$


Visto que $\lim _{\|\bar{y}-\bar{a}\| \rightarrow 0} \frac{\mathcal{R}_{\lambda, \bar{a}}(\bar{y})}{\|\bar{y}-\bar{a}\|^{\lambda}}=0$, então existe $r_{2}>0$ tal que

$$
\left|\mathcal{R}_{\lambda, \bar{a}}(\bar{y})\right|<\|\bar{y}-\bar{a}\|^{\lambda}
$$

para $\|\bar{y}-\bar{a}\| \in] 0, r_{2}[$.

Portanto, para $\|\bar{y}-\bar{a}\| \in] 0, r_{2}[$,

$$
\left|\tilde{f}_{\lambda, \bar{a}}(\bar{y})\right|<|\tilde{f}(\bar{y})|+\|\bar{y}-\bar{a}\|^{\lambda}
$$

De $\bar{a} \in \bar{X}$, temos, pela etapa $\mathrm{C}$, que $\bar{a} \in \bar{G}_{\bar{a}}$. Consequentemente, existe $\bar{b} \in G_{\bar{a}}$ de sorte que

$$
\|\bar{a}-\bar{b}\|<\min \left\{r_{1}, r_{2}\right\}^{6}
$$

De $\bar{b} \in G_{\bar{a}}$, temos que $\phi(\bar{a}, \bar{b})$ vale e $\tilde{f}(\bar{b})=0$. Desta última e de $\|\bar{a}-\bar{b}\|<r_{2}$, segue que

$$
\left|\tilde{f}_{\lambda, \bar{a}}(\bar{b})\right|<\|\bar{b}-\bar{a}\|^{\lambda}
$$

Portanto, por definição, $\bar{b} \in F_{\bar{a}}$.

Por outro lado, seja $\bar{a} \in \bar{F}_{\bar{a}}$ e suponha, por absurdo, que $\bar{a} \notin \bar{X}$.

Então, existem $\gamma_{1}, \gamma_{2}, \gamma_{3}>0$ sendo que:

(a) Para todo $\left.\beta \in] 0, \gamma_{1}\right]$, existe $\bar{y} \in F_{\bar{a}}$ tal que $\|\bar{a}-\bar{y}\|=\beta$.

(b) $d(\bar{a}, X) \geq \gamma_{2}$.

(c) Se $\left.\beta \in] 0, \gamma_{3}\right]$ e $(\beta, \delta) \in B$, então $\beta^{\lambda}+\psi_{f}(\beta)<\delta$, de sorte que $\psi_{f}:[0, \infty[\rightarrow \mathbb{R}$ é uma função dada por

$$
\psi_{f}(t)=\sup \left\{\left|\tilde{f}(\bar{y})-\tilde{f}_{\lambda, \bar{a}}(\bar{y})\right|:\|\bar{y}-\bar{a}\| \leq t\right\} .
$$

\footnotetext{
${ }^{6}$ De $\bar{a} \in \bar{G}_{\bar{a}}$, segue que existe uma sequência $\left(\bar{b}_{j}\right)_{j}$ em $G_{\bar{a}}$ tal que $\bar{b}_{j} \rightarrow \bar{a}$. Logo, para $\min \left\{r_{1}, r_{2}\right\}>0$, existe $J \in \mathbb{N}$ tal que $\left\|\bar{b}_{j}-\bar{a}\right\|<\min \left\{r_{1}, r_{2}\right\}$, para todo $j>J$. Basta, assim, tomar $\bar{b}=\bar{b}_{j}$, para algum $j>J$.
} 
De fato, de $\bar{a} \notin \bar{X}$, temos que existe $s>0$ tal que $\|\bar{x}-\bar{a}\| \geq s$, para todo $\bar{x} \in X$. Logo, $d(\bar{a}, X) \geq s$.

Basta, portanto, tomar $\gamma_{2}=s$.

De (R6a), segue que $\tilde{f}$ é $\mathcal{C}^{\lambda+1}$. Logo, identicamente ao caso da função $\psi_{g}$ na etapa C, temos

$$
\lim _{t \rightarrow 0} \frac{\psi_{f}(t)}{t^{\lambda}}=0
$$

Assim, pela etapa E, existe $\eta>0$ tal que vale a propriedade $\left(4^{*}\right)$, para $\psi_{f}$. Então, tomando $\gamma_{3}=\frac{\eta}{2}$, obtemos a afirmação $(c)$.

Para o caso da existência de $\gamma_{1}$, considere

$$
\mathcal{B}=\left\{\beta: \exists \bar{y}\left(\bar{y} \in F_{\bar{a}} \wedge\|\bar{a}-\bar{y}\|=\beta\right)\right\}
$$

Aqui vale notar que, $\phi(\bar{a}, \bar{y})$ é livre de quantificadores. Pois:

se $\tilde{g}$ é um polinômio em $L$, então $\tilde{g}_{\kappa, \bar{a}}(\bar{y})$ também o é;

se $\tilde{g}$ está em $\mathcal{R}$, então, por (R6c), $\tilde{g}_{\kappa, \bar{a}}(\bar{y})$ é um polinômio em $L \cup\{\tilde{f}: \tilde{f} \in \tilde{R}\}$.

Em ambos os casos, $\tilde{g}_{\kappa, \bar{a}}(\bar{y}) \geq\|\bar{a}-\bar{y}\|^{\kappa}$ é livre de quantificadores e, consequentemente, $\phi(\bar{a}, \bar{y})$ é livre de quantificadores.

Analogamente, $\left|\tilde{f}_{\lambda, \bar{a}}(\bar{y})\right| \leq\|\bar{a}-\bar{y}\|^{\lambda}$ é livre de quantificadores.

Portanto, $\mathcal{B}$ é um conjunto $\exists_{1}$-definível em $\left\langle\overline{\mathbb{R}},\{\tilde{f}\}_{\tilde{f} \in \tilde{R}}\right\rangle$. Em outros termos,

$$
\mathcal{B}=\pi_{n+1,1}[\mathcal{A}]
$$

de sorte que $\mathcal{A}$ é semi-algébrico em $\left\langle\overline{\mathbb{R}},\{\tilde{f}\}_{\tilde{f} \in \tilde{R}}\right\rangle$.

Logo, por (R2),

$$
\mathcal{A}=C_{1} \cup \cdots \cup C_{N},
$$

sendo que $C_{1}, \ldots, C_{N} \subseteq \mathbb{R}^{n+1}$ são conexos. 
Assim,

$$
\mathcal{B}=\underbrace{\pi\left[C_{1}\right]}_{I_{1}} \cup \cdots \cup \underbrace{\pi\left[C_{N}\right]}_{I_{N}}
$$

tal que $I_{1}, \ldots, I_{N} \subseteq \mathbb{R}$ são intervalos reais.

Como $\|\bar{y}-\bar{a}\| \geq 0$, então, existe $i \in\{1, \ldots, N\}$ tal que $I_{i}$ tem 0 (zero) como limitante inferior.

Além disso, $\bar{a} \notin F_{\bar{a}}$.

Caso contrário, $\phi(\bar{a}, \bar{a})$ valeria e $\left|\tilde{f}_{\lambda, \bar{a}}(\bar{a})\right|=0$. Logo, $\tilde{f}_{\lambda, \bar{a}}(\bar{a})=0$. Uma vez que,

$$
\tilde{f}(\bar{x})=\tilde{f}_{\lambda, \bar{a}}(\bar{x})+\mathcal{R}_{\lambda, \bar{a}}(\bar{x}),
$$

com

$$
\left|\mathcal{R}_{\lambda, \bar{a}}(\bar{x})\right| \leq C_{0} \frac{\|\bar{x}-\bar{a}\|^{\lambda+1}}{(\lambda+1) !} \quad \text { e } \quad C_{0}>0
$$

temos que $\mathcal{R}_{\lambda, \bar{a}}(\bar{a})=0$. Assim, $\tilde{f}(\bar{a})=0$. Portanto, pela definição de $G_{\bar{a}}$, teríamos $\bar{a} \in G_{\bar{a}}$. Pela afirmação 2 da etapa $\mathrm{C}$, segue que $\bar{a} \in X \subseteq \bar{X}$. $(\Rightarrow \Leftarrow)$

Portanto, algum dos intervalos $I_{i}$ 's é do tipo

$$
(0, s),(0, s] \text { ou }(0, \infty)
$$

$\operatorname{com} s \in \mathbb{R}$ e $s>0$.

Sem perda de generalidade, assuma que $I_{1}$ seja desta forma.

Se $I_{1}$ é do primeiro ou do segundo tipo, tome $\gamma_{1}=\frac{s_{1}}{2}$.

Se $\left.I_{1}=\right] 0, \infty\left[\right.$, basta tomar um real $\gamma_{1}>0$ arbitrário.

Assim, para todo $\left.\beta \in] 0, \gamma_{1}\right] \subseteq I_{1}$ segue que $\beta \in \mathcal{B}$ e, por definição, existe $\bar{y} \in F_{\bar{a}}$ tal que $\|\bar{a}-\bar{y}\|=\beta$.

Agora, seja $\gamma=\min \left\{\gamma_{1}, \gamma_{2}, \gamma_{3}\right\}$.

Fixe $\beta \in] 0, \gamma]$. 
Considere o compacto $^{7}$

$$
\mathcal{W}_{\beta}=\{\bar{y}: \phi(\bar{a}, \bar{y}) \wedge\|\bar{a}-\bar{y}\|=\beta\}
$$

Então, por $(a), \mathcal{W}_{\beta} \neq \emptyset$. Além disso, a função $\bar{y} \mapsto|\tilde{f}(\bar{y})|$ é contínua. Portanto, $\min \left\{|\tilde{f}(\bar{y})|: \bar{y} \in \mathcal{W}_{\beta}\right\}$ existe. Também,

$$
\mathcal{W}_{\beta} \subseteq\{\bar{y}:\|\bar{a}-\bar{y}\|=\beta\} \subseteq\{\bar{y}:\|\bar{a}-\bar{y}\| \leq \gamma\}
$$

para todo $\beta \in] 0, \gamma]$.

Chamemos $\{\bar{y}:\|\bar{a}-\bar{y}\| \leq \gamma\}$ de $\mathcal{W}$.

Como $\mathcal{W}$ é compacto, seja $m_{\mathcal{W}}=\max \{|\tilde{f}(\bar{y})|: \bar{y} \in \mathcal{W}\}$. Então,

$$
\min \left\{|\tilde{f}(\bar{y})|: \bar{y} \in \mathcal{W}_{\beta}\right\} \leq m_{\mathcal{W}}
$$

para todo $\beta \in] 0, \gamma]$.

Ou seja, o conjunto $\left.\left.\left\{\min \left\{|\tilde{f}(\bar{y})|: \bar{y} \in \mathcal{W}_{\beta}\right\}: \beta \in\right] 0, \gamma\right]\right\}$ é limitado superiormente. Logo, possui supremo.

Seja

$$
\delta=\sup _{\beta \in] 0, \gamma]} \min \left\{|\tilde{f}(\bar{y})|: \bar{y} \in \mathcal{W}_{\beta}\right\}
$$

Por $(b)$ e pela definição de $B($ Etapa $\mathrm{D})$, segue que $(\gamma, \delta) \in B$.

Como $\left.\gamma \in] 0, \gamma_{3}\right]$, então, por $(c)$,

$$
\gamma^{\lambda}+\psi_{f}(\gamma)<\delta\left(=\sup _{\beta \in] 0, \gamma]} \min \left\{|\tilde{f}(\bar{y})|: \bar{y} \in \mathcal{W}_{\beta}\right\}\right)
$$

Consequentemente, existe $\left.\left.\beta_{0} \in\right] 0, \gamma\right]$ de sorte que

$$
\gamma^{\lambda}+\psi_{f}(\gamma)<\min \left\{|\tilde{f}(\bar{y})|: \bar{y} \in \mathcal{W}_{\beta_{0}}\right\} .
$$

\footnotetext{
${ }^{7}$ Note que $\mathcal{W}_{\beta}=\left\{\bar{y}: \tilde{g}_{\kappa, \bar{a}}(\bar{y}) \geq\|\bar{a}-\bar{y}\|^{\kappa} \wedge \tilde{g}(\bar{a})>0 \wedge\|\bar{a}-\bar{y}\|=\beta\right\}$.
} 
Pela definição de $\psi_{f}$, temos

$$
\left|\tilde{f}(\bar{y})-\tilde{f}_{\lambda, \bar{a}}(\bar{y})\right| \leq \psi_{f}(\gamma)
$$

para todo $\bar{y} \in \mathcal{W}$.

Em particular, para todo $\bar{y} \in \mathcal{W}_{\beta_{0}}$,

$$
|\tilde{f}(\bar{y})|-\left|\tilde{f}_{\lambda, \bar{a}}(\bar{y})\right| \leq\left|\tilde{f}(\bar{y})-\tilde{f}_{\lambda, \bar{a}}(\bar{y})\right| \leq \psi_{f}(\bar{y})
$$

o que implica

$$
\begin{aligned}
\psi_{f}(\bar{y})+\gamma^{\lambda} \geq|\tilde{f}(\bar{y})|-\left|\tilde{f}_{\lambda, \bar{a}}(\bar{y})\right|+\gamma^{\lambda} & \geq \min _{\bar{y} \in \mathcal{W}_{\beta_{0}}}\left(|\tilde{f}(\bar{y})|-\left|\tilde{f}_{\lambda, \bar{a}}(\bar{y})\right|+\gamma^{\lambda}\right) \\
& >\psi_{f}(\bar{y})+\gamma^{\lambda}-\left|\tilde{f}_{\lambda, \bar{a}}(\bar{y})\right|+\gamma^{\lambda}
\end{aligned}
$$

Portanto,

$$
\left|\tilde{f}_{\gamma, \bar{a}}(\bar{y})\right|>\gamma^{\lambda} \geq \beta_{0}^{\lambda}
$$

para todo $\bar{y} \in \mathcal{W}_{\beta_{0}}$.

Logo, pela definição de $F_{\bar{a}}$, não existe $\bar{y} \in F_{\bar{a}}$ tal que $\|\bar{y}-\bar{a}\|=\beta_{0}$. Contradição com $(a)$.

Portanto, se $\bar{a} \in F_{\bar{a}}$, então $\bar{a} \in \bar{X}$.

Etapa $G$ :

Seja $\sigma(\bar{x}, \bar{y}, \varepsilon)$ a fórmula

$$
\left(\tilde{g}_{\kappa, \bar{x}}(\bar{y}) \geq\|\bar{x}-\bar{y}\|^{\kappa}\right) \wedge(\tilde{g}(\bar{x})>0 \vee\|\bar{x}-\bar{y}\|>0) \wedge\left(\left|\tilde{f}_{\lambda, \bar{x}}(\bar{y})\right| \leq\|\bar{y}-\bar{x}\|^{\lambda}\right) \wedge(\|\bar{y}-\bar{x}\|<\varepsilon) .
$$

Então, da etapa $\mathrm{F}$, vemos facilmente que

$$
\bar{X}=\{\bar{x}: \forall \varepsilon>0 \exists \bar{y} \sigma(\bar{x}, \bar{y}, \varepsilon)\}
$$


Note que as variáveis $\bar{y}$ e $\varepsilon$ ocorrem apenas polinomialmente em $\sigma$.

Seja $\tilde{L}=L \cup\{\tilde{f}: \tilde{f} \in \tilde{R}\}$. Então, $\tilde{g}(\bar{x})$ e $\tilde{f}_{1}(\bar{x}), \ldots, \tilde{f}_{l}(\bar{x})$ são termos de $\tilde{L}$. Por (R6c), segue que

$$
\frac{\partial^{j_{1}+\cdots+j_{n}} \tilde{g}}{\partial x_{1}^{j_{1}} \cdots \partial x_{n}^{j_{n}}}(\bar{x}),
$$

para $0 \leq j_{1}+\cdots+j_{n} \leq \kappa, \mathrm{e}$

$$
\frac{\partial^{j_{1}+\cdots+j_{n}} \tilde{f}_{i}}{\partial x_{1}^{j_{1}} \cdots \partial x_{n}^{j_{n}}}(\bar{x}),
$$

para $0 \leq j_{1}+\cdots+j_{n} \leq \lambda$ e $i=1, \ldots, l$, são também termos de $\tilde{L}$.

Denotemos por $\tilde{T}$ o conjunto de todos estes termos. Então, substituímos cada $t(\bar{x}) \in \tilde{T}$ por uma variável $w_{t(\bar{x})} \in \operatorname{Var}$. Como $|\tilde{T}|<\omega$ e $|V a r|=\omega$, então podemos tomar as variáveis $w_{t(\bar{x})}$ diferentes de todas que ocorrem em $\sigma$ e diferentes entre si, $i$. e., $w_{t(\bar{x})} \neq w_{s(\bar{x})}$, para $t(\bar{x}) \neq s(\bar{x})$. Assim, teremos

$$
\left\langle\overline{\mathbb{R}},\{\tilde{f}\}_{\tilde{f} \in \tilde{R}}\right\rangle \models \forall \varepsilon>0 \exists \bar{y} \sigma(\bar{x}, \bar{y}, \varepsilon) \leftrightarrow \forall \varepsilon>0 \exists \bar{y} \exists \tilde{w} \sigma^{\prime}(\bar{x}, \tilde{w}, \bar{y}, \varepsilon),
$$

de sorte que $\sigma^{\prime}(\bar{x}, \tilde{w}, \bar{y}, \varepsilon)$ é uma fórmula semi-algébrica de $L$.

Seja $R C O F$ a teoria dos corpos ordenados reais fechados. Uma vez que $R C O F$ tem eliminação de quantificadores (em $L$ ), segue que existe $\psi(\bar{x}, \tilde{w})$ aberta de $L$ tal que

$$
R C O F \models \forall \varepsilon>0 \exists \bar{y} \exists \tilde{w} \sigma^{\prime}(\bar{x}, \tilde{w}, \bar{y}, \varepsilon) \leftrightarrow \psi(\bar{x}, \tilde{w})
$$

Seja $\psi^{\prime}(\bar{x})$ a fórmula aberta de $\tilde{L}$ obtida de $\psi$ através da substituição de $w_{t(\bar{x})}$ por $t(\bar{x})$. Então, em $\langle\overline{\mathbb{R}},\{\tilde{f}\}\rangle_{\tilde{f} \in \tilde{R}}$,

$$
\forall \varepsilon>0 \exists \bar{y} \sigma(\bar{x}, \bar{y}, \varepsilon) \leftrightarrow \psi^{\prime}(\bar{x})
$$

Portanto, $\bar{X}$ é definível, em $\langle\overline{\mathbb{R}},\{\tilde{f}\}\rangle_{\tilde{f} \in \tilde{R}}$, sem quantificadores.

Antes de enunciarmos e provarmos o último teorema deste trabalho, vale registrar o principal resultado de [9]: 
Teorema 3.1.4. (Gabrielov, 1996) Seja uma coleção $\mathcal{F}$ de funções analíticas a valores reais cujos dominios são vizinhanças abertas de $[-1,1]^{n}$ em $\mathbb{R}^{n}$, para variados $n$ 's, e suponha que $\mathcal{F}$ seja fechada sob diferenciação parcial. Então, a teoria de $\left\langle\overline{\mathbb{R}},\left\{f^{*}: f \in \mathcal{F}\right\}\right\rangle$ é modelo completa, sendo que $f^{*}: \mathbb{R}^{n} \rightarrow \mathbb{R}$ é definida como

$$
f^{*}(\bar{x})=\left\{\begin{array}{cl}
f(\bar{x}), & \text { se } \bar{x} \in[-1,1]^{n} \\
0, & \text { caso contrário }
\end{array}\right.
$$

O teorema subsequente (Teorema 3.1.5) é uma consequência do Teorema 3.1.3 e uma generalização do Teorema 3.1.4.

Teorema 3.1.5. Suponha que $R$ satisfaz as condições $(R 2)$, $(R 5)$ e (R6). Então, a teoria $T h(\langle\overline{\mathbb{R}},\{f: f \in R\}\rangle)$ é modelo completa.

Demonstração. Por hipótese, $(R 6 a)$ vale e, portanto, $(R 1)$ é satisfeita. Logo, pelo Teorema 3.1.2, basta mostrarmos que $(R 4)$ é válida. Seja $W \subseteq[-1,1]^{n}$, um conjunto dado por

$$
W=\left\{\bar{x} \in[-1,1]^{n}: f(\bar{x})=0 \wedge \bigwedge_{\sigma(i)=+\infty}\left(x_{i}>0\right) \wedge \bigwedge_{\sigma(i)=-\infty}\left(x_{i}<0\right)\right\}
$$

de sorte que $f$ é da forma $p\left(\bar{x}, f_{1}(\bar{x}), \ldots, f_{l}(\bar{x})\right)$, com $f_{1}, \ldots, f_{l} \in R$ e $p \in \mathbb{R}\left[z_{1}, \ldots, z_{n+l}\right]$.

Considere os polinômios $g_{i}$ 's,

$$
g_{i}(\bar{x})=\left\{\begin{array}{rl}
x_{i}, & \text { se } \sigma(i)=+\infty \\
-x_{i}, & \text { se } \sigma(i)=-\infty
\end{array} .\right.
$$

Assim, podemos reescrever $W$ como

$$
W=\left\{\bar{x} \in[-1,1]^{n}: f(\bar{x})=0 \wedge \bigwedge_{\sigma(i) \neq 0} g_{i}(\bar{x})>0\right\} .
$$

Sejam $\tilde{f}_{1}, \ldots, \tilde{f}_{l}$ as restrições de $f_{1}, \ldots, f_{l}$, respectivamente, ao cubo $[-1,1]^{n}$, de sorte que elas se anulam em $\mathbb{R}^{n} \backslash[-1,1]^{n}$. Considere, ainda, $\tilde{f}=p\left(\bar{x}, \tilde{f}_{1}(\bar{x}), \ldots, \tilde{f}_{l}(\bar{x})\right)$. 
Agora, note que, em $[-1,1]^{n}$,

$$
\begin{aligned}
W & =\left\{\bar{x} \in[-1,1]^{n}:\left\langle\overline{\mathbb{R}},\{f\}_{f \in R}\right\rangle \models f(\bar{x})=0 \wedge \bigwedge_{\sigma(i) \neq 0} g_{i}(\bar{x})>0\right\} \\
& =\left\{\bar{x} \in[-1,1]^{n}:\left\langle\overline{\mathbb{R}},\{\tilde{f}\}_{\tilde{f} \in \tilde{R}}\right\rangle \models \tilde{f}(\bar{x})=0 \wedge \bigwedge_{\sigma(i) \neq 0} g_{i}(\bar{x})>0\right\}=W_{1} .
\end{aligned}
$$

Consequentemente, $\bar{W}=\overline{W_{1}}$.

Pelo Teorema 3.1.3, $\overline{W_{1}}$ é definido por uma fórmula sem quantificadores em $\left\langle\overline{\mathbb{R}},\{\tilde{f}\}_{\tilde{f} \in \tilde{R}}\right\rangle$, digamos $\phi(\bar{x}, \vec{v})$. Então, substituindo em cada ocorrência de $\tilde{f}_{j}$, em $\phi$, pelo seu correspondente $f_{j}$, obtemos uma fórmula aberta $\phi^{\prime}$.

Uma vez que, os valores das variáveis $\bar{x}$ em $\phi(\bar{x}, \vec{v})$ e $\phi^{\prime}(\bar{x}, \vec{v})$ são tomados em $[-1,1]^{n}$, então, novamente, $\tilde{f}_{j}$ e $f_{j}$ coincidem. Em outros termos, para todo $\bar{x} \in[-1,1]^{n}$,

$$
\left\langle\overline{\mathbb{R}},\{\tilde{f}\}_{\tilde{f} \in \tilde{R}}\right\rangle \models \phi(\bar{x}, \vec{v}) \text { se, e somente se }\left\langle\overline{\mathbb{R}},\{f\}_{f \in R}\right\rangle \models \phi^{\prime}(\bar{x}, \vec{v}) .
$$

Portanto, $\bar{W}$ é definível sem quantificadores. Em particular, $\bar{W}$ é $\exists_{1}$-definível em $\left\langle\overline{\mathbb{R}},\{f\}_{f \in R}\right\rangle$.

Note que, a fim de concluirmos que o Teorema 3.1.4 é uma consequência do Teorema 3.1.5, é preciso, obviamente, que uma dada expansão $\left\langle\overline{\mathbb{R}},\left\{f^{*}: f \in \mathcal{F}\right\}\right\rangle$, como no resultado de Gabrielov (Teorema 3.1.4), satisfaça as hipóteses (R2), (R5) e (R6). Entretanto, a obtenção, por parte desta expansão, de algumas de tais propriedades não é trivial. Torna-se, então, profíqua a seguinte descrição: as expansões $\left\langle\overline{\mathbb{R}},\left\{f^{*}: f \in \mathcal{F}\right\}\right\rangle$ e $\mathbb{R}_{a n}{ }^{8}$ possuem os mesmos conjuntos definíveis; por [4] e [8], $\mathbb{R}_{a n}$ satisfaz (R6b) e é o-minimal, então, pelo Teorema 3.1.1, $\mathbb{R}_{a n}$ satisfaz (R2); logo, $\left\langle\overline{\mathbb{R}},\left\{f^{*}: f \in \mathcal{F}\right\}\right\rangle$ tem também tais propriedades. Além disso, uma expansão obtida por funções restritas (como no Teorema 3.1.4) e uma obtida pelas funções não restritas correspondentes, mas que satisfaz (R5), são essencialmente as mesmas ${ }^{9}$. Isto

\footnotetext{
${ }^{8} \mathbb{R}_{a n}$ é a expansão de $\overline{\mathbb{R}}$ obtida pela adição de todas as funções analíticas (restritas) da forma (†), como no Teorema 3.1.4.

${ }^{9} \mathrm{Ou}$ seja, têm os mesmos conjuntos definíveis.
} 
completa a verificação, para expansão $\left\langle\overline{\mathbb{R}},\left\{f^{*}: f \in \mathcal{F}\right\}\right\rangle$ em questão, das propriedades não imediatas.

Concluímos este trabalho observando que tanto o Teorema 3.1.5 quanto o Teorema 3.1.2 implicam a o-minimalidade da estrutura $\left\langle\overline{\mathbb{R}},\{f\}_{f \in R}\right\rangle$, desde que as condições (R2), (R5) e (R6) sejam satisfeitas. Nesta ocasião, vale mencionar o trabalho [23] de Wilkie, que pode ser resumido da seguinte forma: dada uma expansão $\widetilde{\mathbb{R}}$ de $\mathbb{R}$ por funções $\mathcal{C}^{\infty}$, tal que a coleção dos conjuntos definíveis sem quantificadores em $\widetilde{\mathbb{R}}$ satisfaz (EF5), então $\widetilde{\mathbb{R}}$ é o-minimal. Em outros termos, para ele, são necessárias apenas hipóteses (R2) e (R6a). Nesta mesma direção, em [10], Karpinski e Macintyre mostram que a o-minimalidade de $\widetilde{\mathbb{R}}$ é ainda obtida, mesmo quando as funções adicionadas são $\mathcal{C}^{n}$, para todo $n$. 


\section{Referências Bibliográficas}

[1] Bianconi, R. Notas de aula do curso Teoria dos modelos e aplicações ministrado pelo Prof. Ricardo Bianconi em 2009. Endereço: http://www.ime.usp.br/ bianconi/mat5865/.

[2] Chang, C. C., Keisler, H. Jerome, Model theory, 3rd Ed., Elsevier Science Publishers, Amsterdam, 1990.

[3] Charbonnel, J. -Y., Sur certains sous-ensembles de l'espace euclidien, Ann. Inst. Fourier Grenoble 41 (3) (1991) 679-717.

[4] Dries, L. van den, A generalization of the Tarski-Seidenberg theorem and some nondefinability results, Bull. of the AMS, Vol. 15, No. 2 (Oct., 1986) 189-193.

[5] Dries, L. van den, Remarks on Tarski's problem concerning $\langle\mathbb{R},+, \cdot$, exp $\rangle$, in Logic Colloquium '82, G. Lolli, G. Longo, and A. Marcja, eds., North-Holland (1984), 187-195.

[6] Dries, L. van den, Tame topology and o-minimal structures, Cambridge University Press, Cambridge, 1998.

[7] Douwen, E. K. van, Fubini's theorem for null sets, The American Math. Month., Vol. 96, No. 8 (1989), 718-721.

[8] Gabrielov, A., Projections of semianalytic sets, Funct. Anal. Appl., 2 (1968), 282-291.

[9] Gabrielov, A., Complements of subanalytic sets and existential formulas for analytic functions, Invent. Math, 125 (1) (1996) 1-12. 
[10] Karpinski, M. and Macintyre, A., A generalization of Wilkie's theorem of the complement, and an application to Pfaffian closure, Sel. Math., New ser. 5 (1999) 507-516.

[11] Knight, J., Pillay, A., Steinhorn, C., Definable sets in ordered structures I, Trans. of the AMS, Vol. 295, No. 2 (Jun., 1986) 565-592.

[12] Knight, J., Pillay, A., Steinhorn, C., Definable sets in ordered structures II, Trans. of the AMS, Vol. 295, No. 2 (Jun., 1986) 593-605.

[13] Krantz, Steven G., Real analysis and foundations, CRC Press, Boca Raton, 2005.

[14] Lima, Elon Lages, Espaços métricos, Coleção Projeto Euclides, Rio, 2003.

[15] Marker, D., Model theory: an introduction, Springer-Verlag, New York, 2002.

[16] Maxwell, S., A general model completeness result for expansions of the real ordered field, Ann. Pure Appl. Logic 95 (1998) 185-227.

[17] Oxtoby, J. C., Measure and category, Springer-Verlag, Berlin, 1971.

[18] Shoenfield, J. R., Mathematical logic, CRC Press, Boca Raton, 2010.

[19] Servi, T., On the first order theory of real exponentiation, Tesi di perfezionamento, 2006.

[20] Tarski, A, A decision method for elementary algebra and geometry, 2nd Ed. revised, Rand Corporation, Berkeley and Los Angeles, 1951.

[21] Wilkie, A. J., Some remarks on a paper of Charbonnel, preprint, University of Oxford, 1994.

[22] Wilkie, A. J., Model completeness results for expansions of the ordered field of real numbers by restricted Pfaffian functions and the exponential function, J. Amer. Math. Soc. 9 (1996) 1051-1094.

[23] Wilkie, A. J., A theorem of the complement and some new o-minimal structures, Sel. Math., New ser. 5 (1999) 397-421. 


\section{Îndice Remissivo}

álgebra, 37

aridade, 6

assinatura, 5

bijeção linear, 20

$C h-$

fórmula, 41, 52, 57, 76, 77, 85, 96, 115, 125

classe elementar, 10

complexidade, 42,43

da fórmula, 8

de um termo, 7

componentes conexas, 18, 22, 23, 31-33, 35, $36,45-49,51,55,73,74,101,110,111$, $120,124,139,140$

conjunto

$\exists_{1}$-definível, 12

afim, 20, 23, 25, 32, 33, 45, 46, 51, 55, 74, 101, 120, 139, 140

de símbolos de variáveis, 6

definível, 11, 12

definível com parâmetros, 11 existencialmente definível, 12, 124

Lebesgue mensurável, 19, 44

semi-algébrico, 20

consequência lógica, 10

cubo, 81-84, 86, 89, 96, 104, 109, 111, 169 aberto, 20, 49, 51, 52, 54-56, 59-61, 81, 84, 85, 87-91, 96, 97, 100, 103, 109, $111,113,118$

fechado, 20, 84

decomposição

celular, 18, 69-71, 73-80, 134

diagrama, 14

eliminação de quantificadores, 13

espaço vetorial, 37

estrutura, 5, 6, 9-13

domínio de uma, 6

fraca, 18, 20, 21, 23, 26, 29

fraca o-minimal, 2, 18, 21, 38, 40, 124, 130, 134

o-minimal, iii, 1, 13, 17, 124, 125, 136, 139, 140, 171

expansão, iii, 1, 2, 9, 18, 124, 133, 137, 139, 
171

de Taylor, 149, 154, 162

extensão, 7

elementar, 11

fórmula, 7

aberta, 9

existencial, 9, 12, 14, 125, 135, 136

livre de quantificadores, 9

prenexa, 9

sem quantificadores, 9

universal, 9, 14

atômica, 8

fecho

Charbonnel, 2, 3, 18, 37, 55

imersão, 7

intervalo degenerado, 45, 48

isomorfismo, 7

linguagem

de primeira ordem, 6

dos anéis, 12

medida

de Lebesgue, 44

nula, 18, 19, 44, 53, 60, 83, 87, 105, 107

positiva, 44, 61, 87

modelo, 9

existencialmente fechado, 14

morfismo, 6

elementar, 11 ordem linear densa, 10

sem extremos, 13

pré-estrutura, 19, 37

pseudofunção, 87-90, 105-108

reduto, 9,140

símbolo

de constante, 5

de função, 5

predicativo, 5

seleção

de uma função $\mathcal{C}^{p}, 81$

de uma função contínua, 81

sentença, 8

subespaço afim, 20, 22, 23, 25, 28, 29, 31, 32, 35

subestrutura, 7

elementar, 11

$T-$

equivalente, 14

Teorema

da Alfândega, 72

da Completude de Gödel, 9

da Decomposição Celular, 54, 70, 134

de Bolzano-Weierstrass, 48, 58, 95, 151

de Fubini, 44, 47, 53, 56, 77, 87

de Weierstrass, 33, 112, 121

do Complemento, 3, 18, 114

do Valor Intermediário, 86, 103 
do Valor Médio, 92

teoria, 9

completa, 10

consistente, 9

da estrutura $M, 10$

decidível, 1

fechada sob consequência lógica, 10

maximal, 10

modelo completa, iii, 1, 3, 14, 15, 18, 124,

134, 136, 139, 140, 142, 169

que admite eliminação de quantificadores,

13

termo, 7

variável

ligada, 8

livre, 8 